Supplement of Atmos. Chem. Phys., 14, 8579-8597, 2014

http://www.atmos-chem-phys.net/14/8579/2014/

doi:10.5194/acp-14-8579-2014-supplement

(C) Author(s) 2014. CC Attribution 3.0 License.

(c) (i)

Supplement of

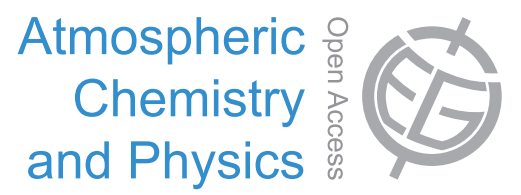

\title{
A climatology of dust emission events from northern Africa using long- term surface observations
}

S. M. Cowie et al.

Correspondence to: S. M. Cowie (eesc@leeds.ac.uk) 

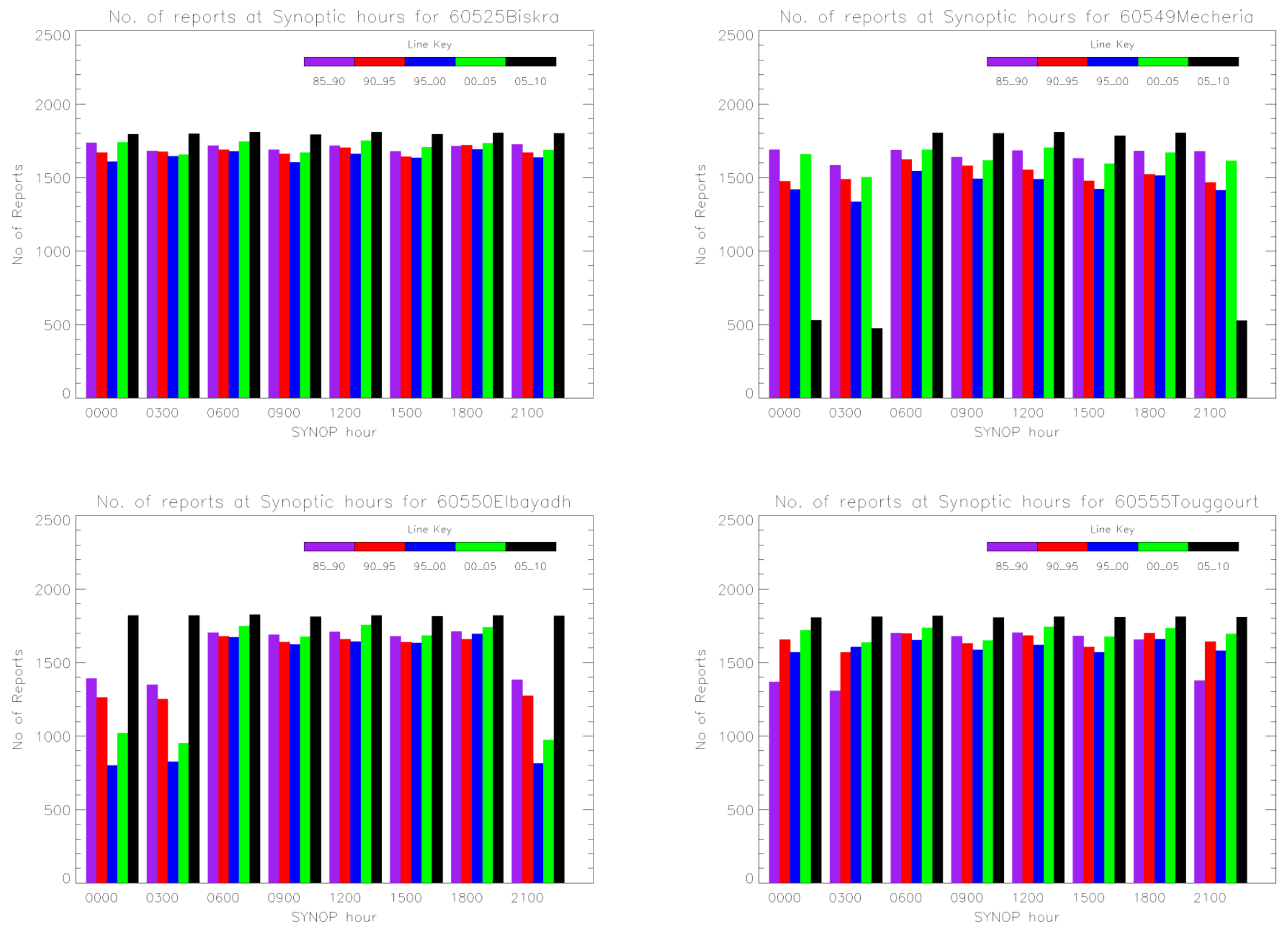

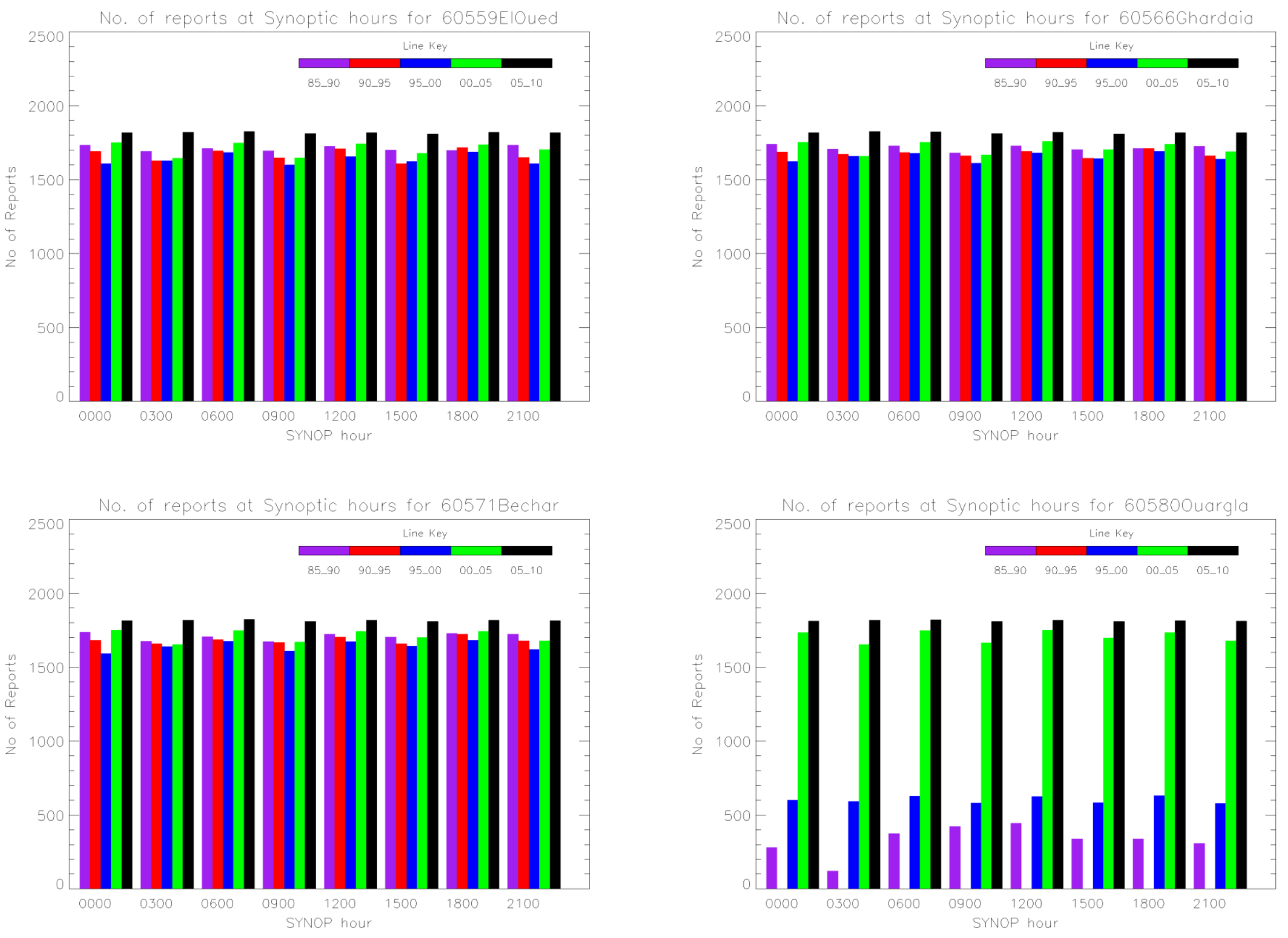

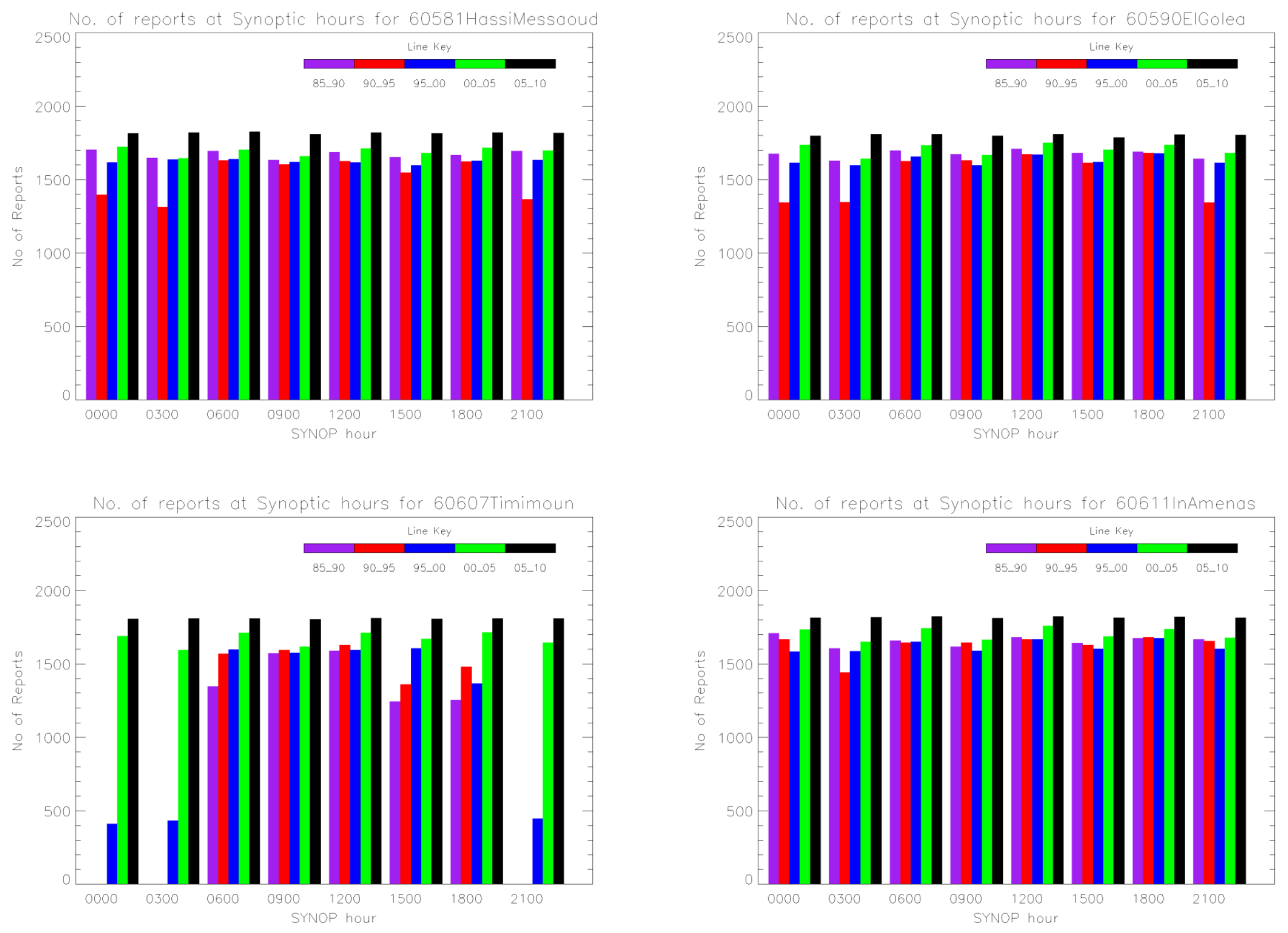

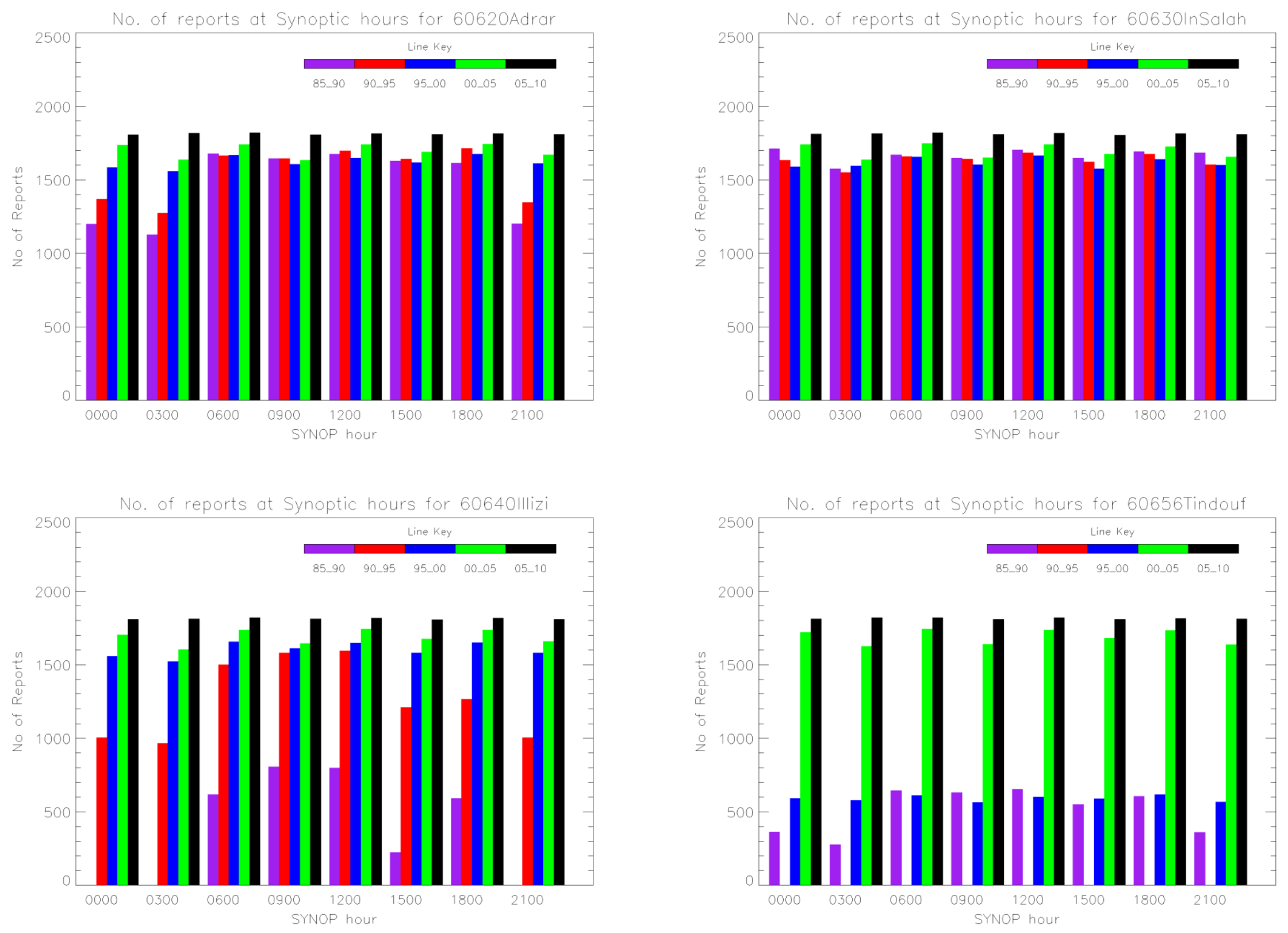

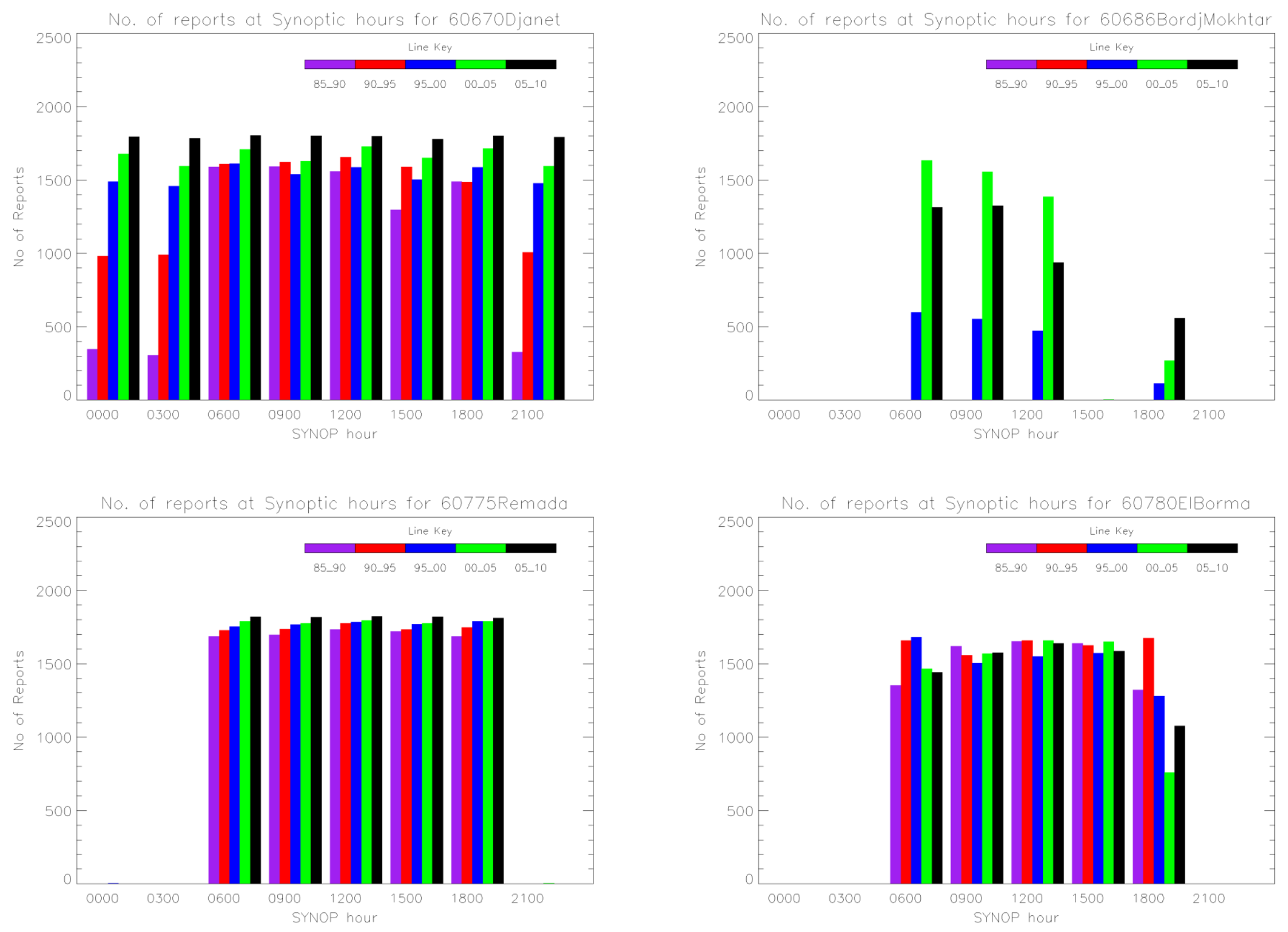

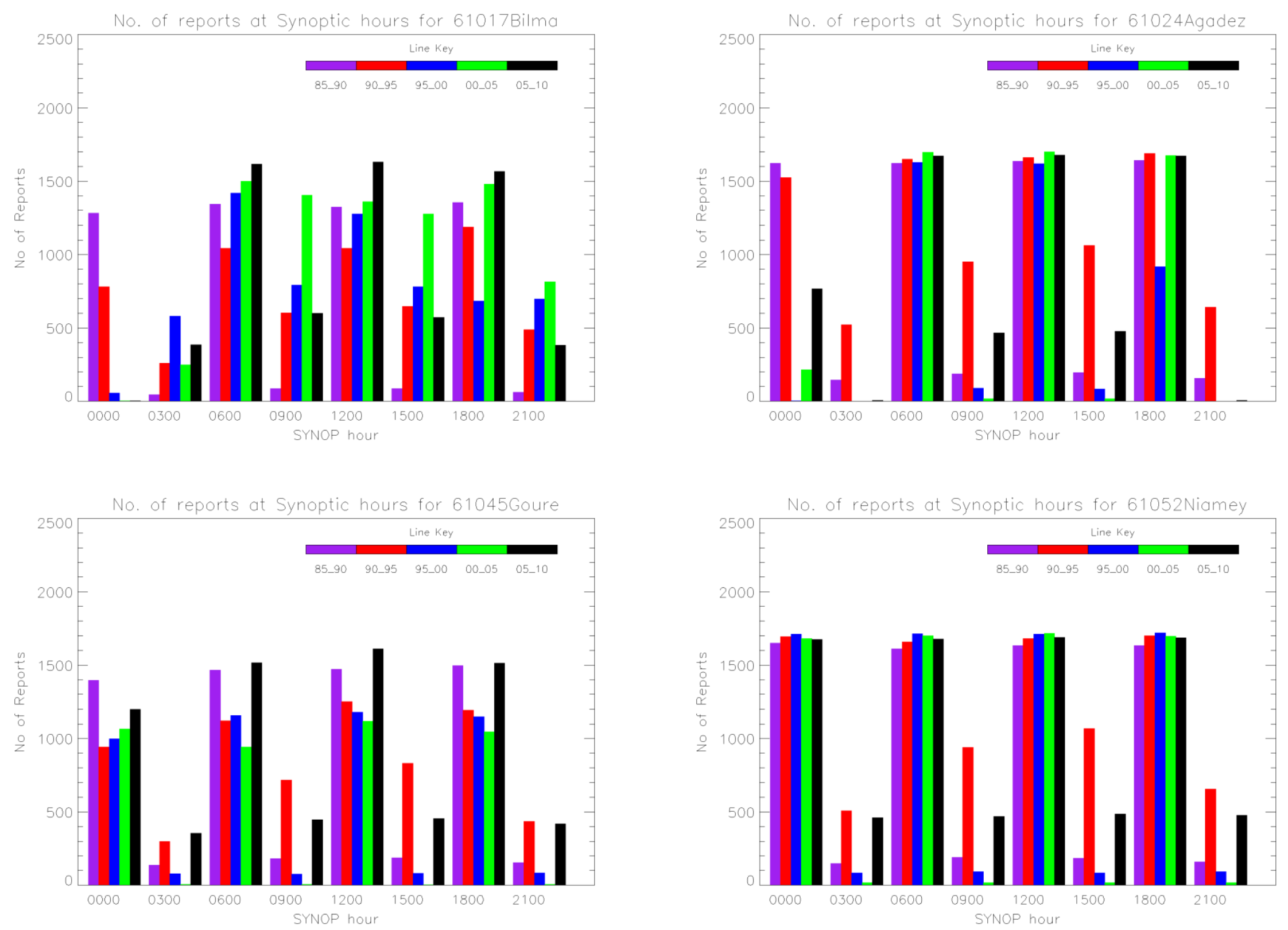

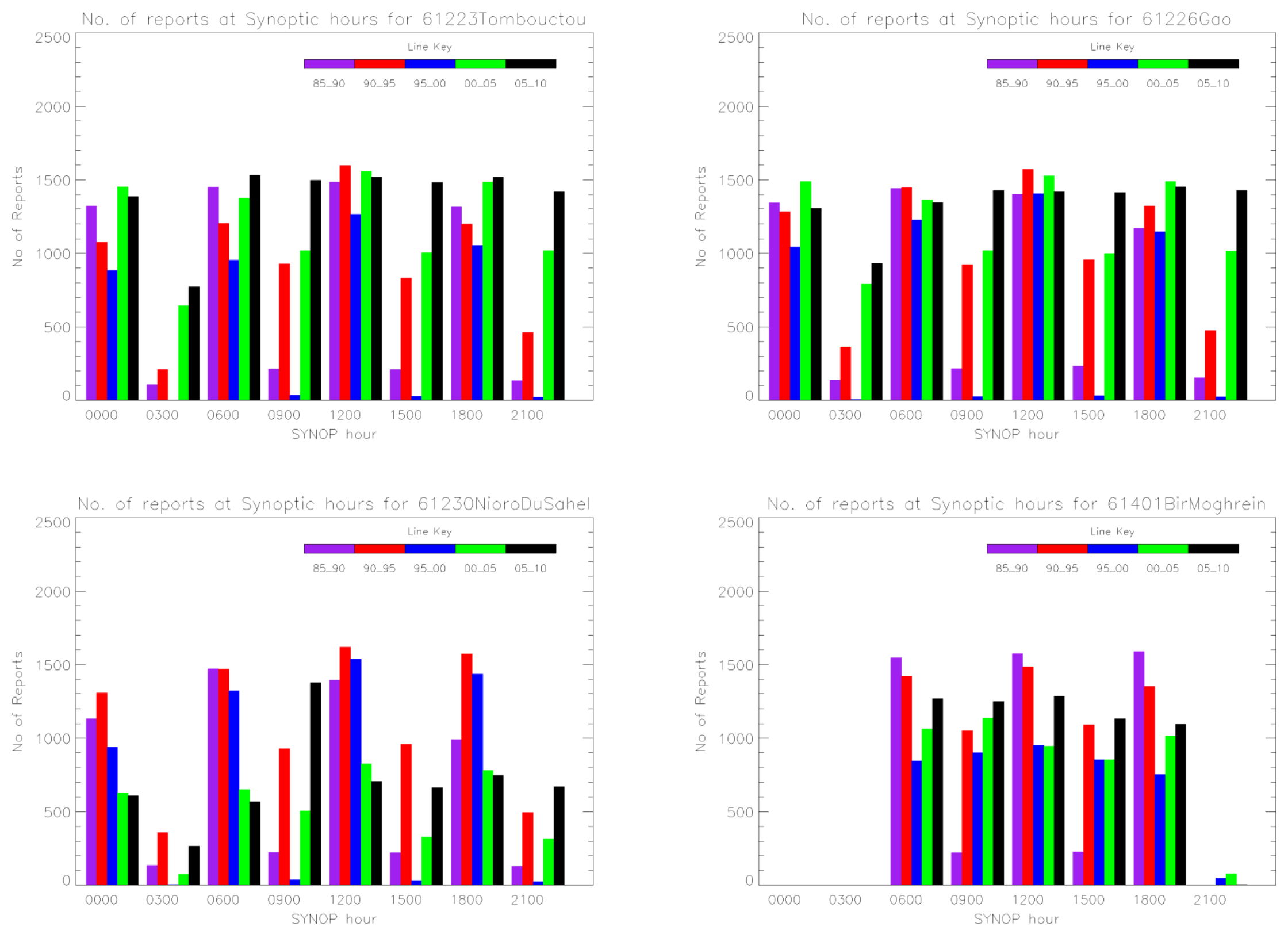

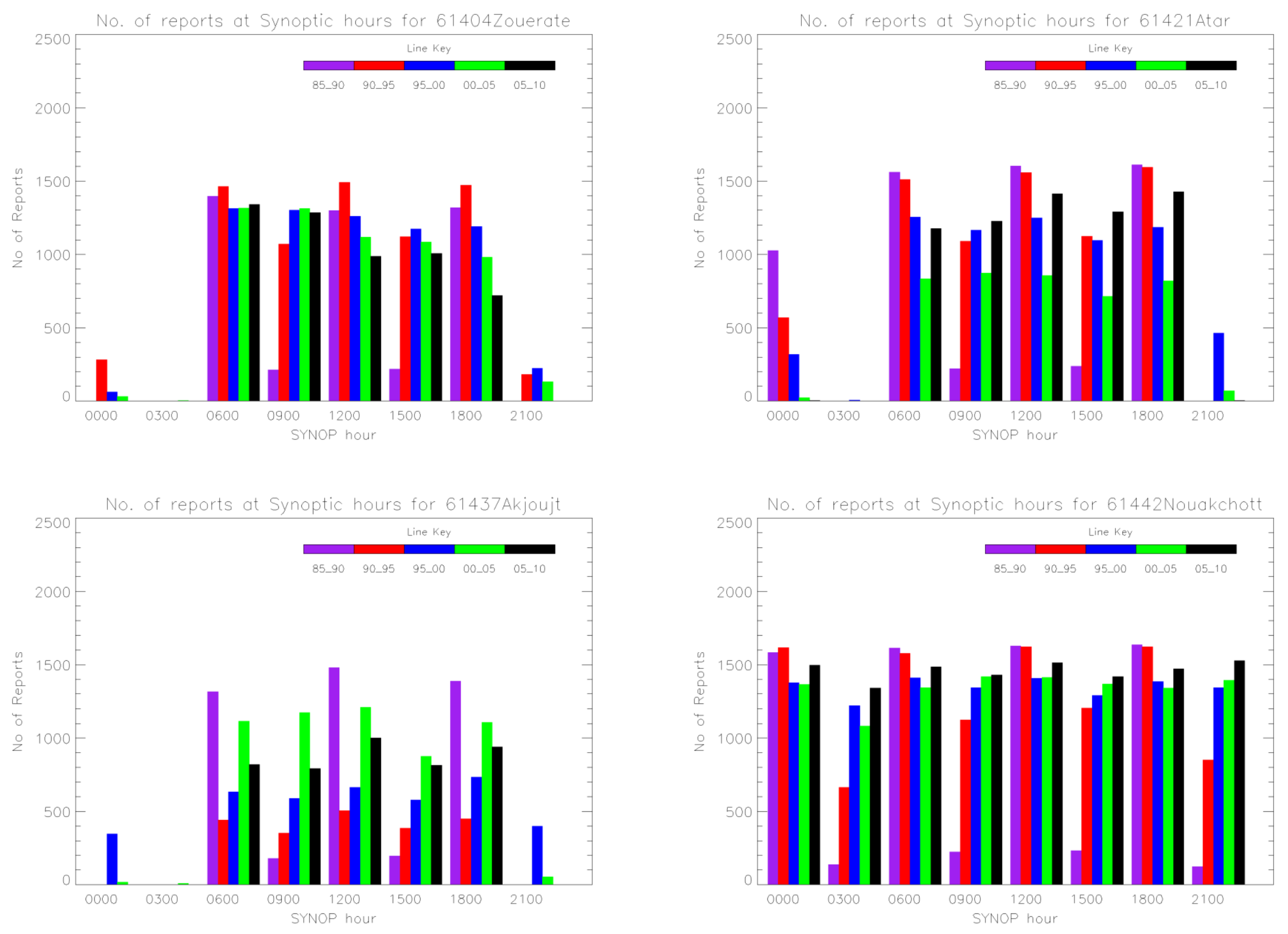

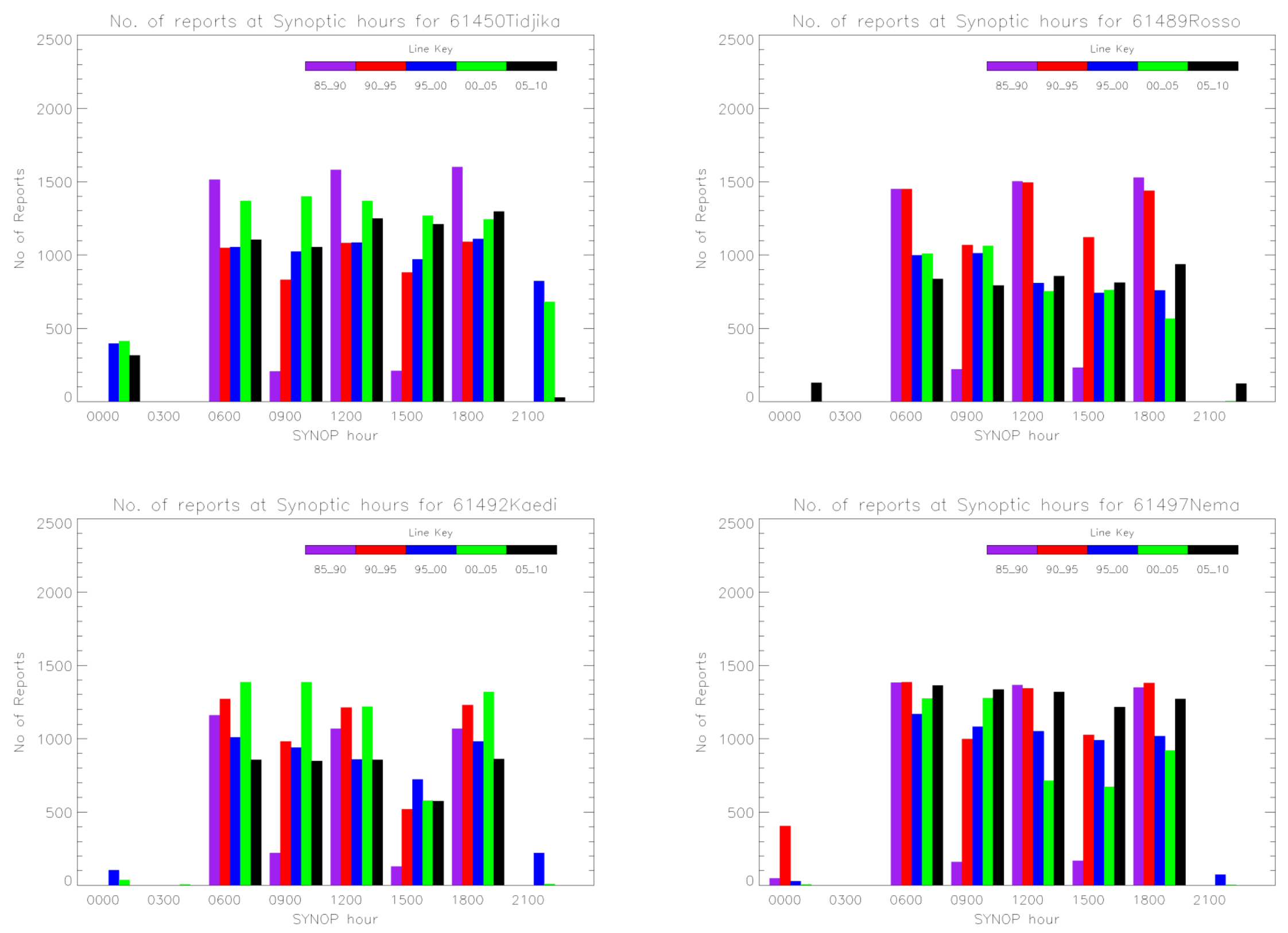

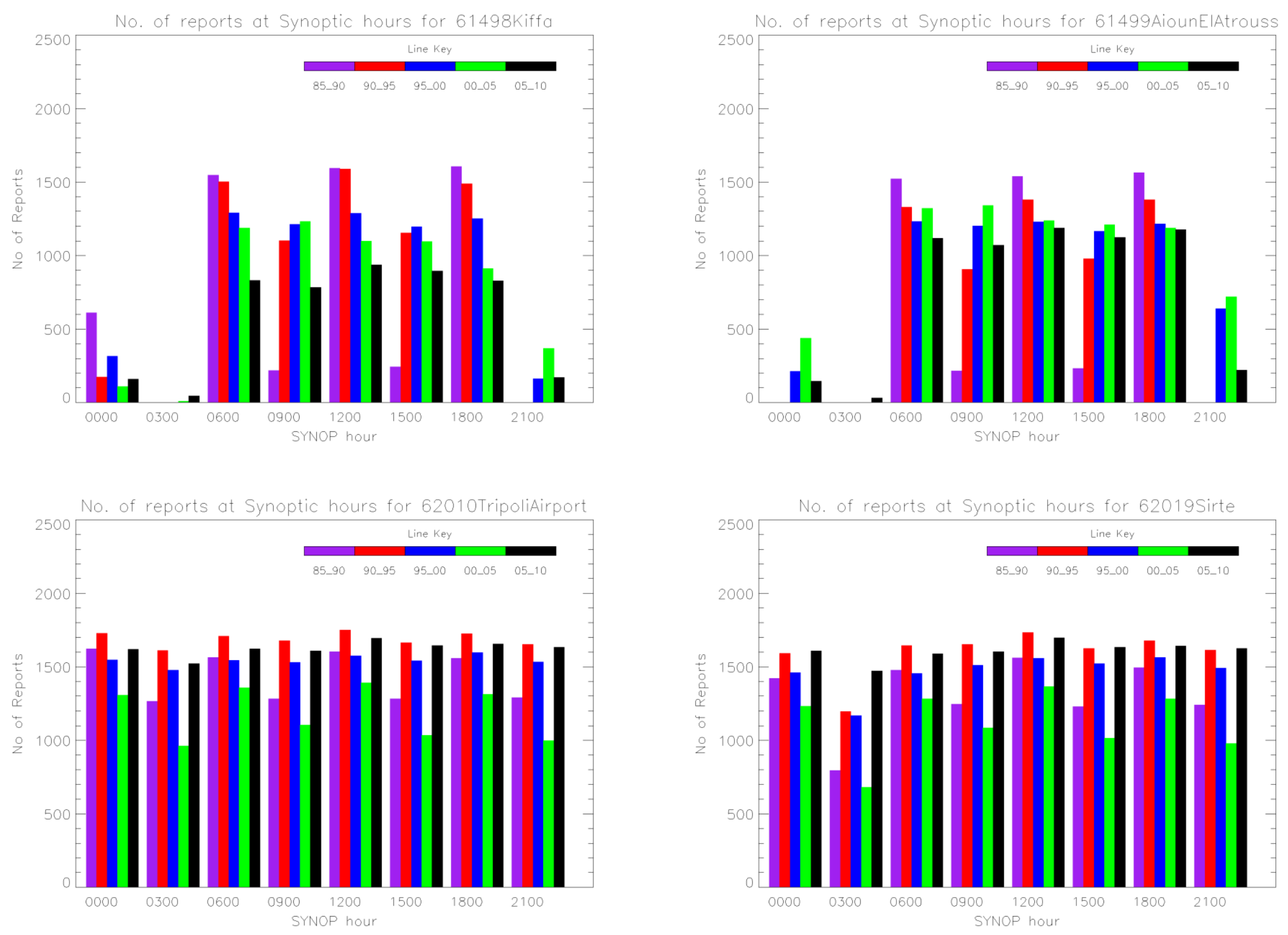

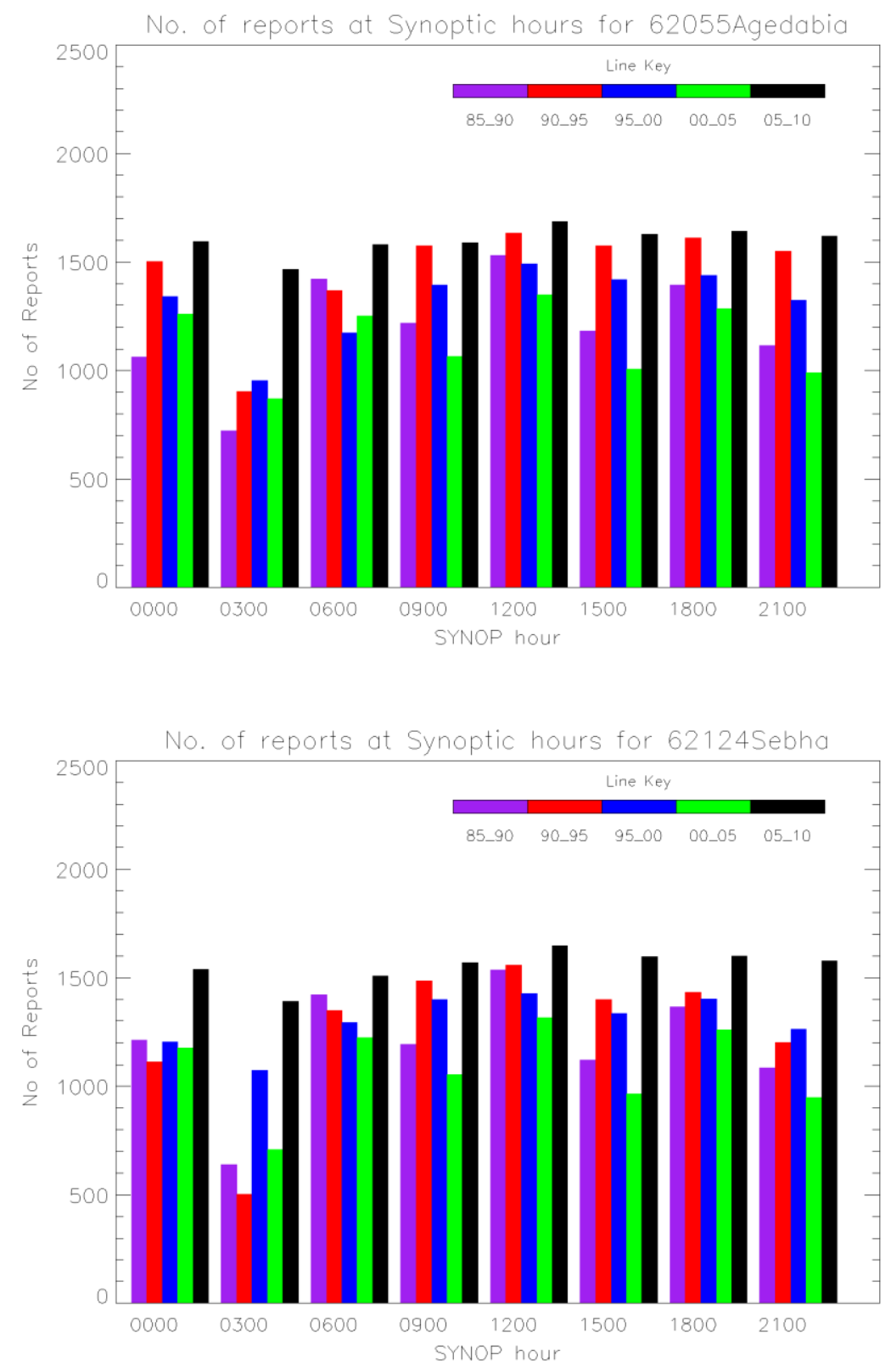
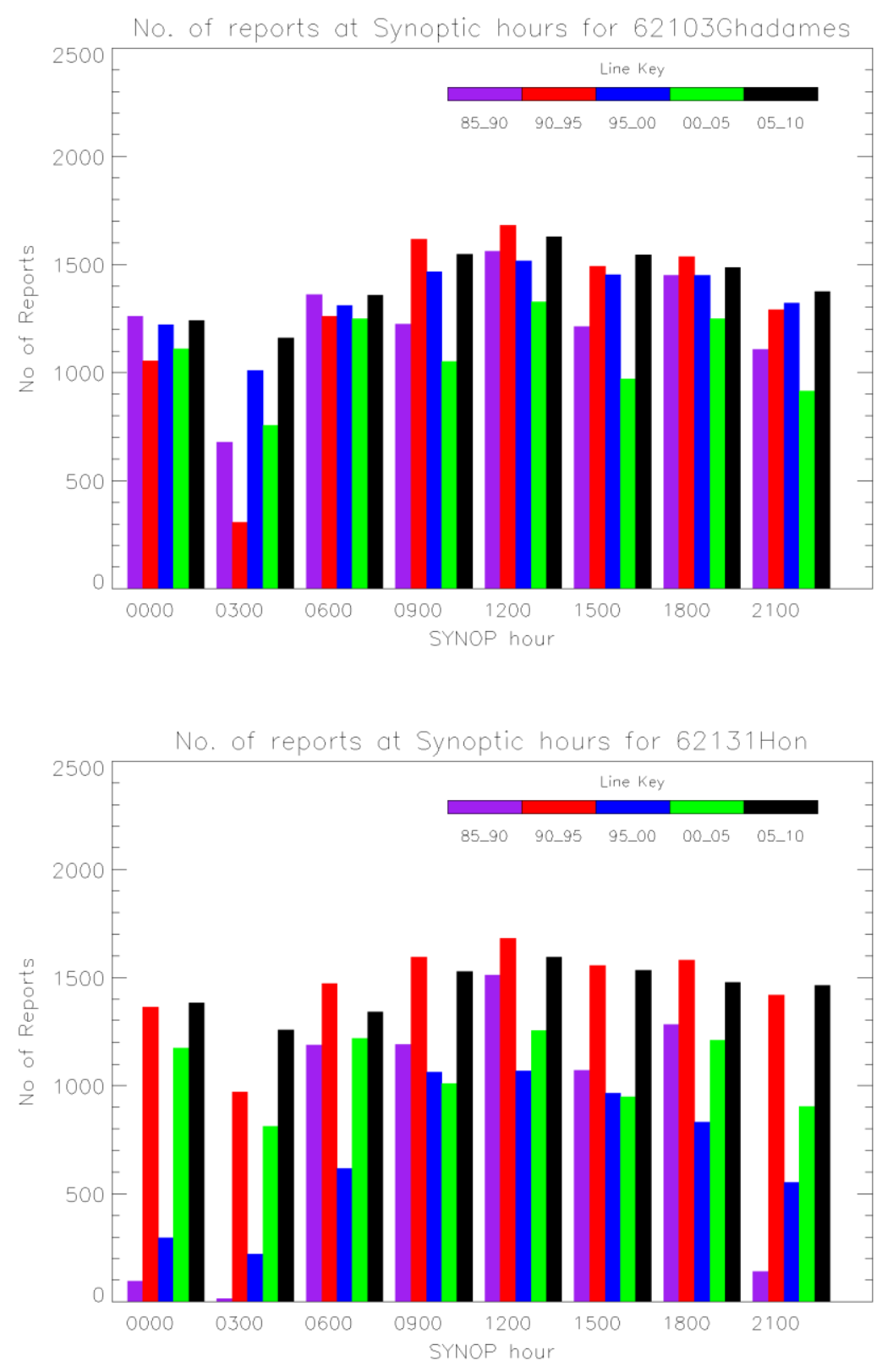

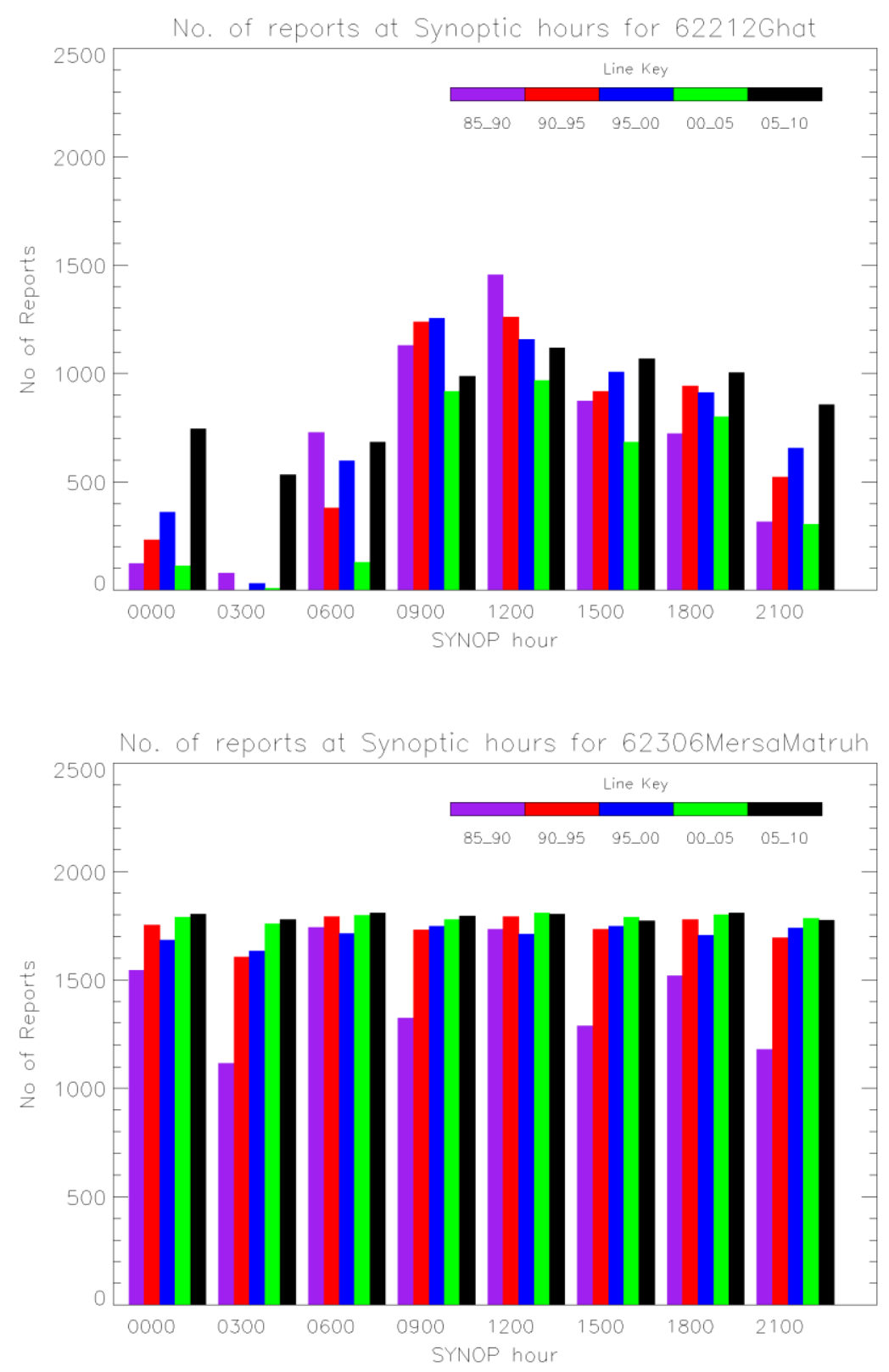
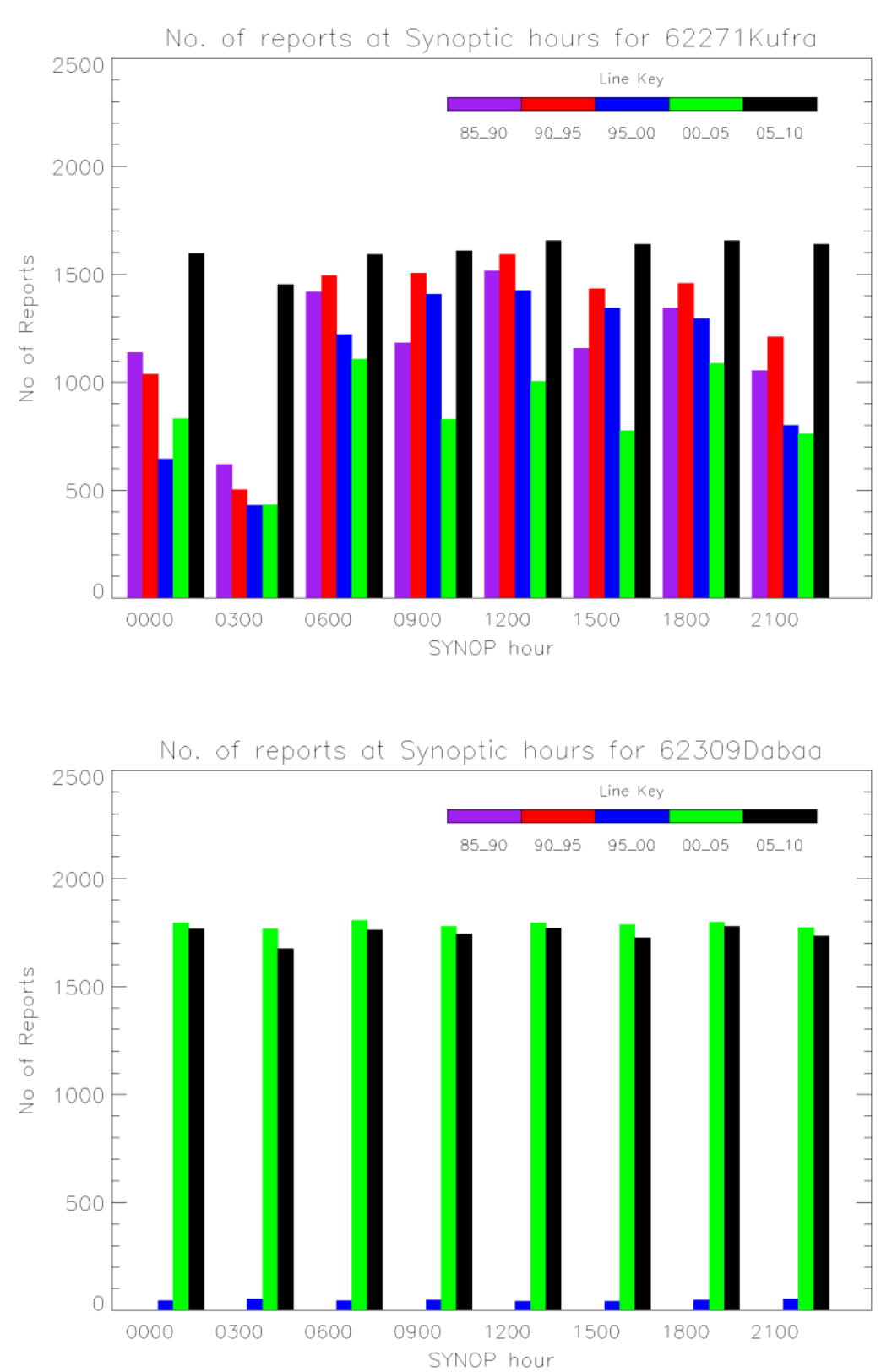

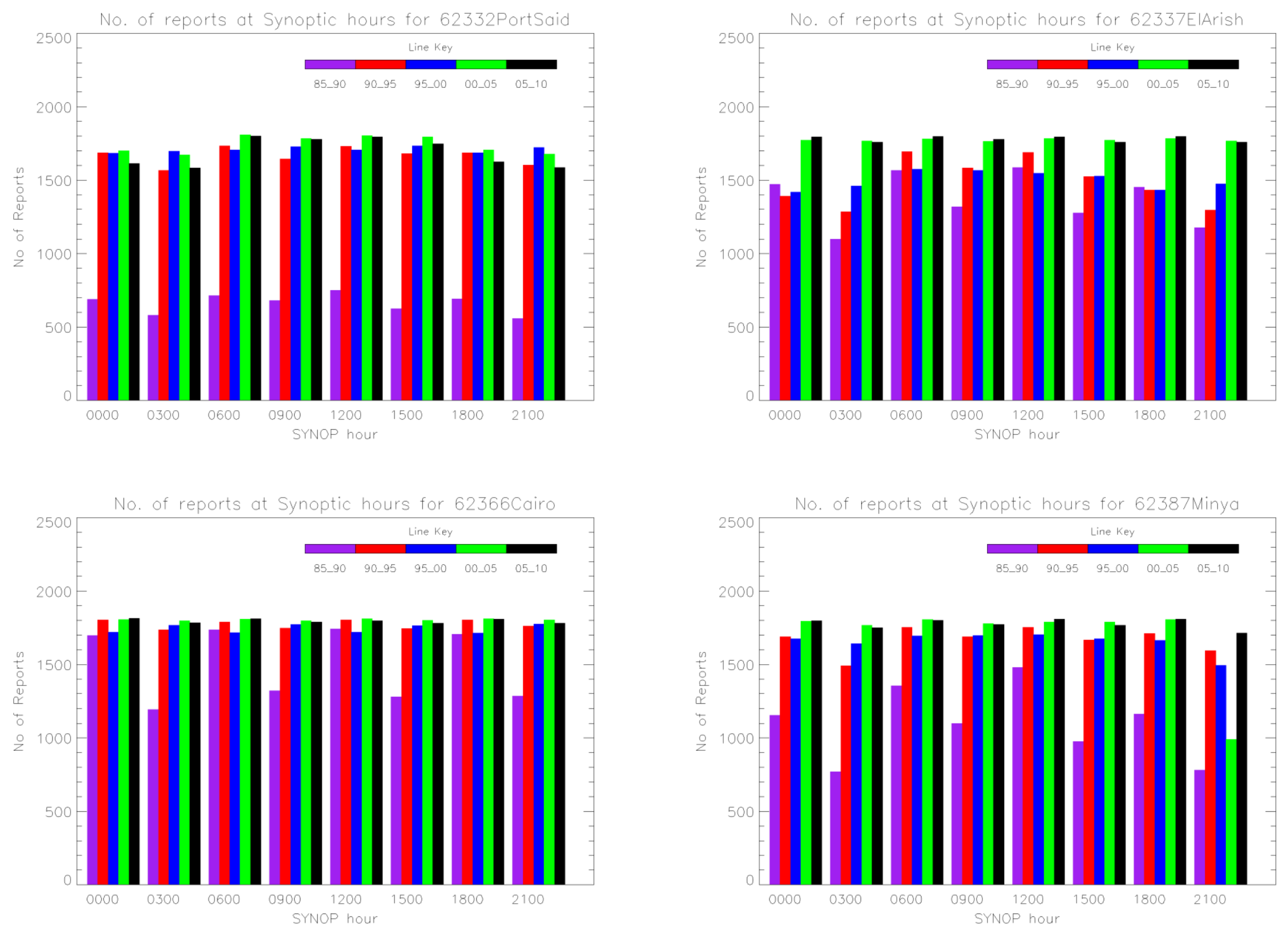

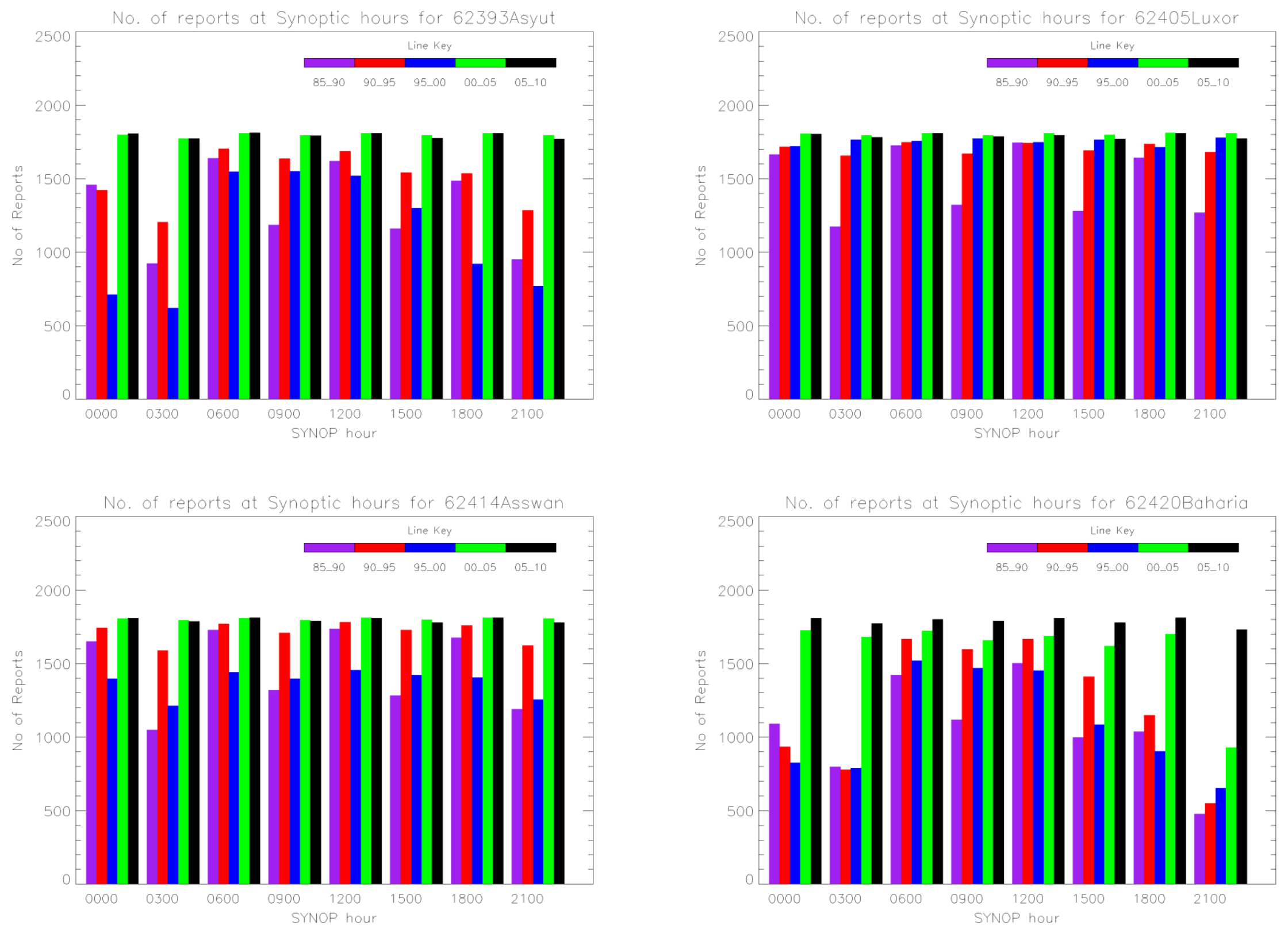

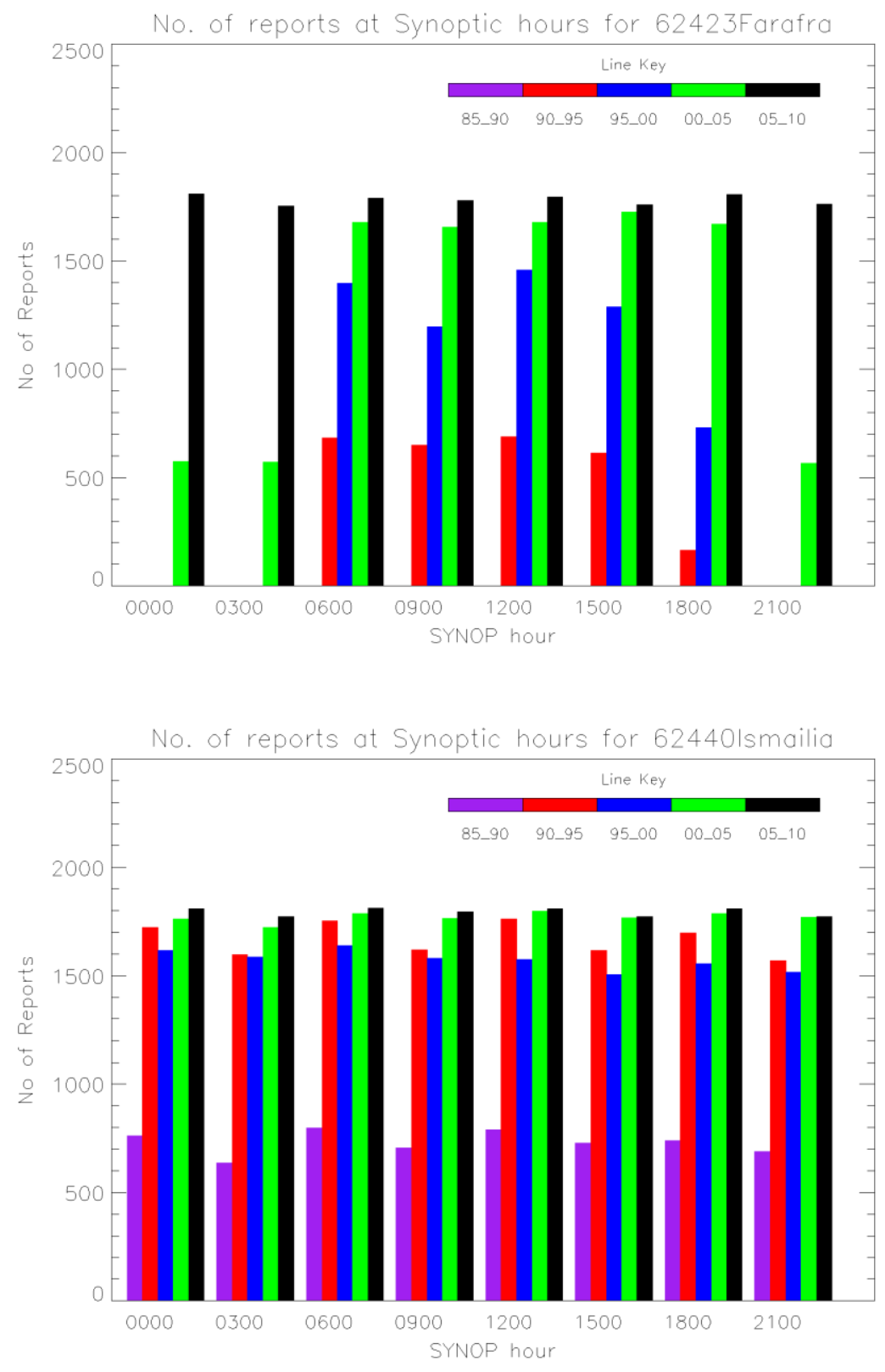
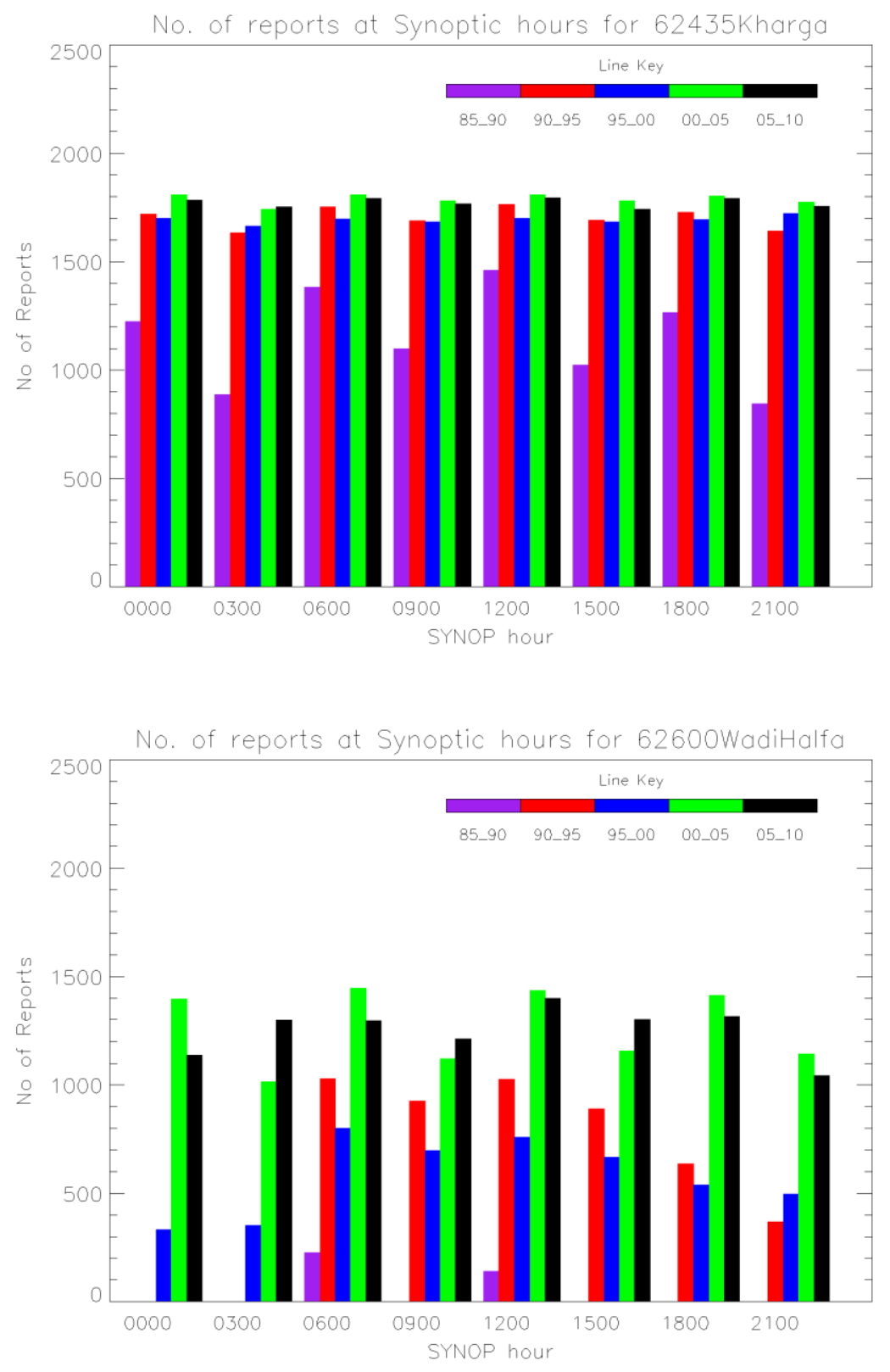

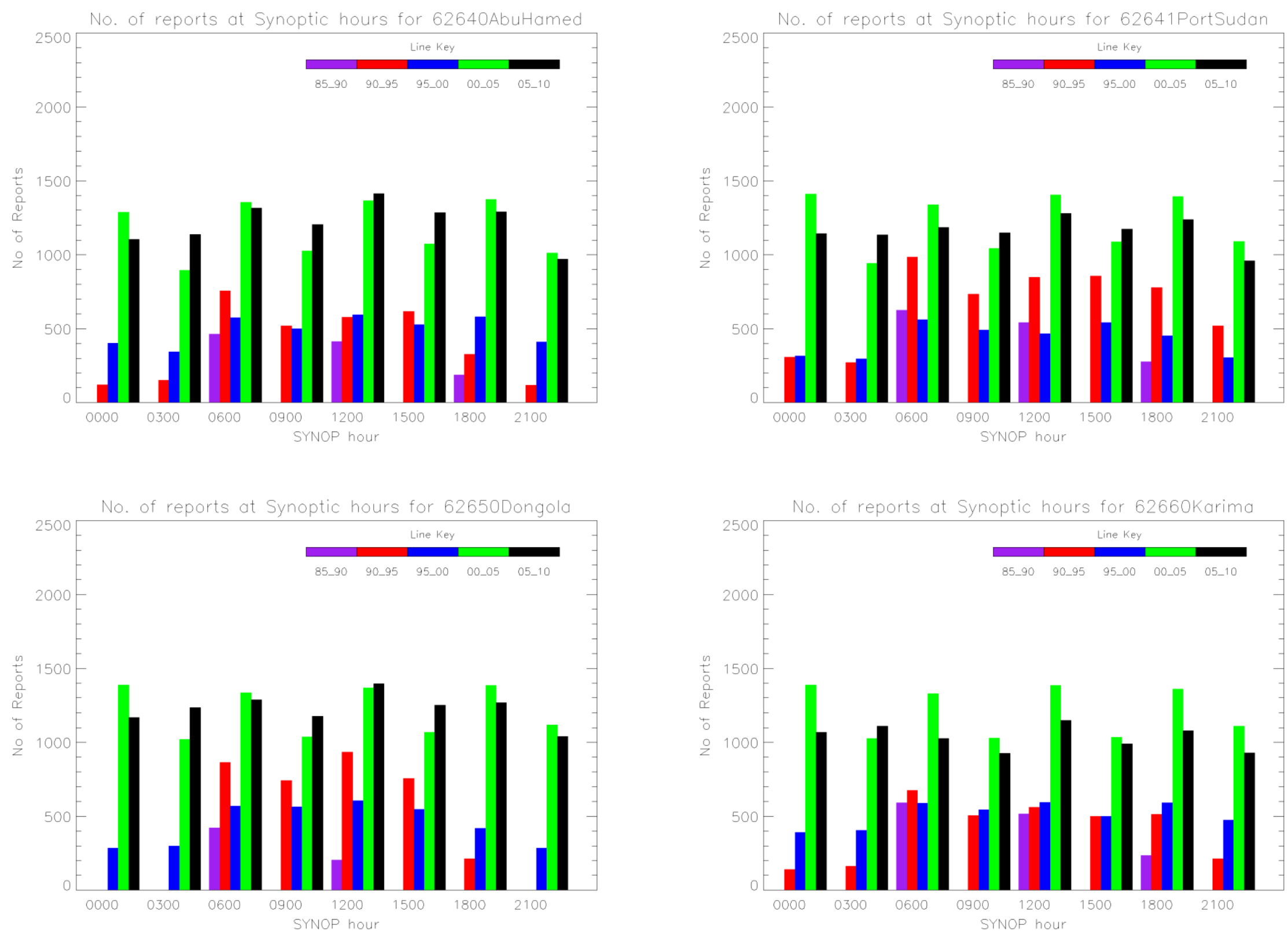

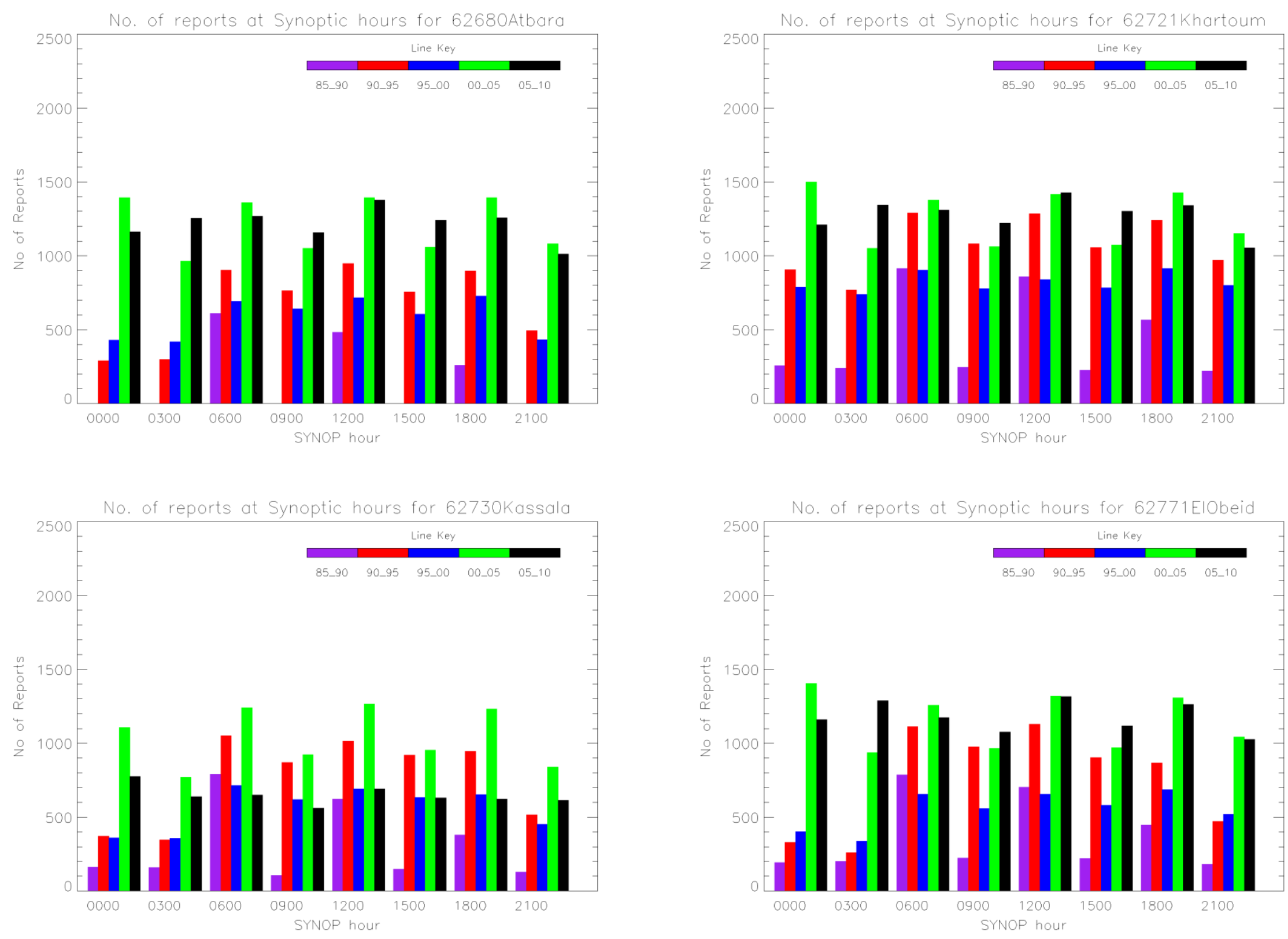

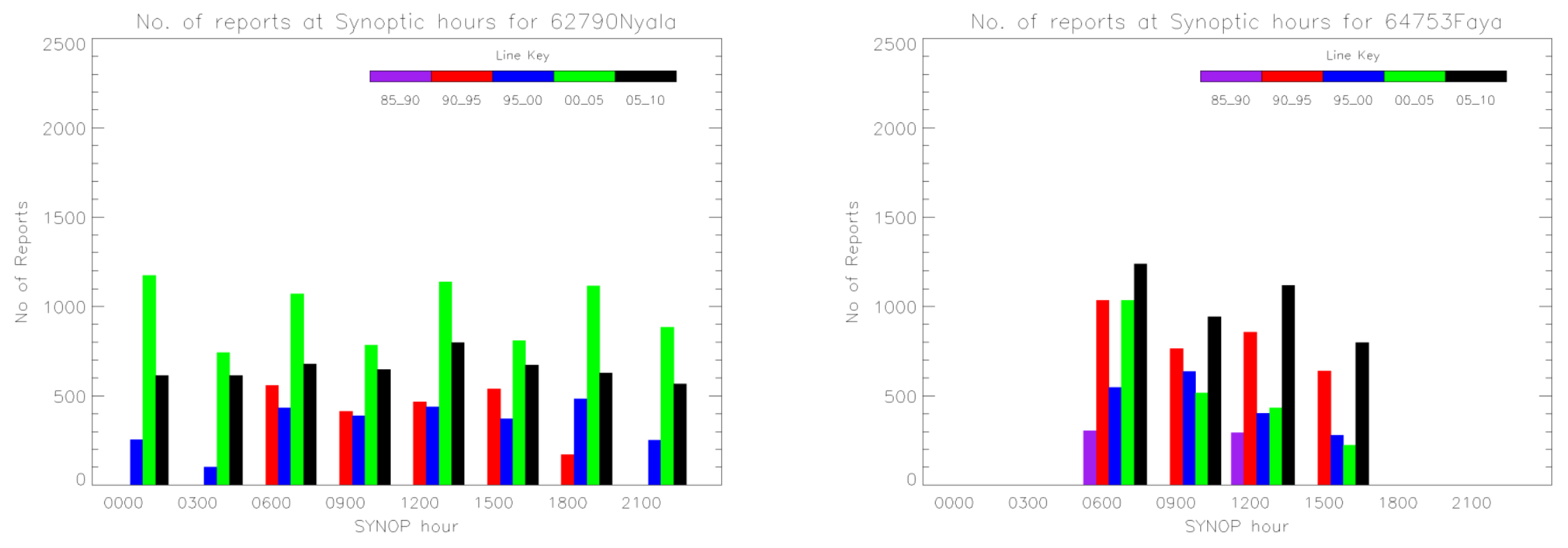

Figure S1. Bar plots of the number of observations at each SYNOP hour, for the periods 1985-1990 (purple), 1990-1995 (red), 1995-2000 (blue), 2000-2005 (green), 2005-2010 (black) for each of the 70 stations. 

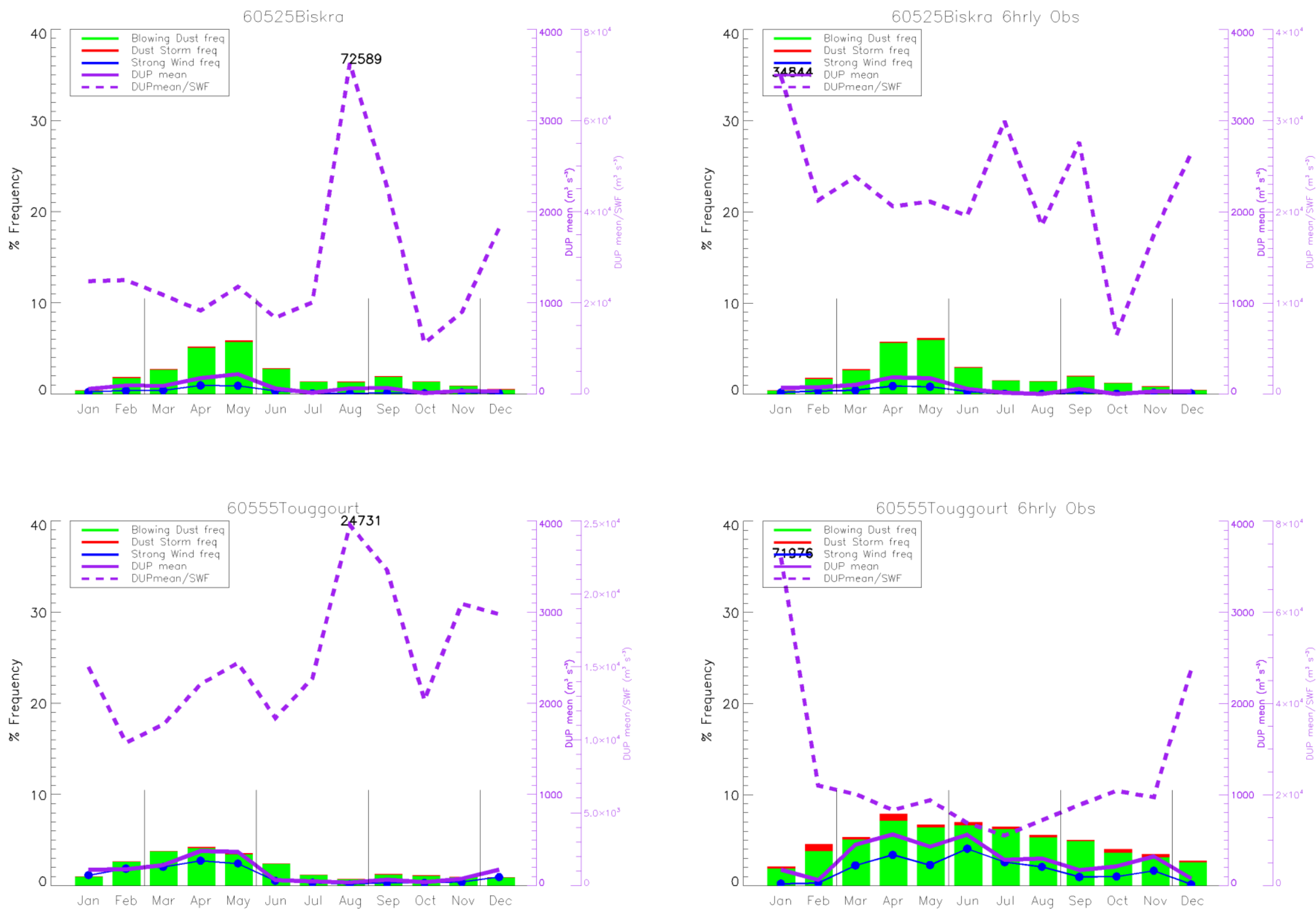

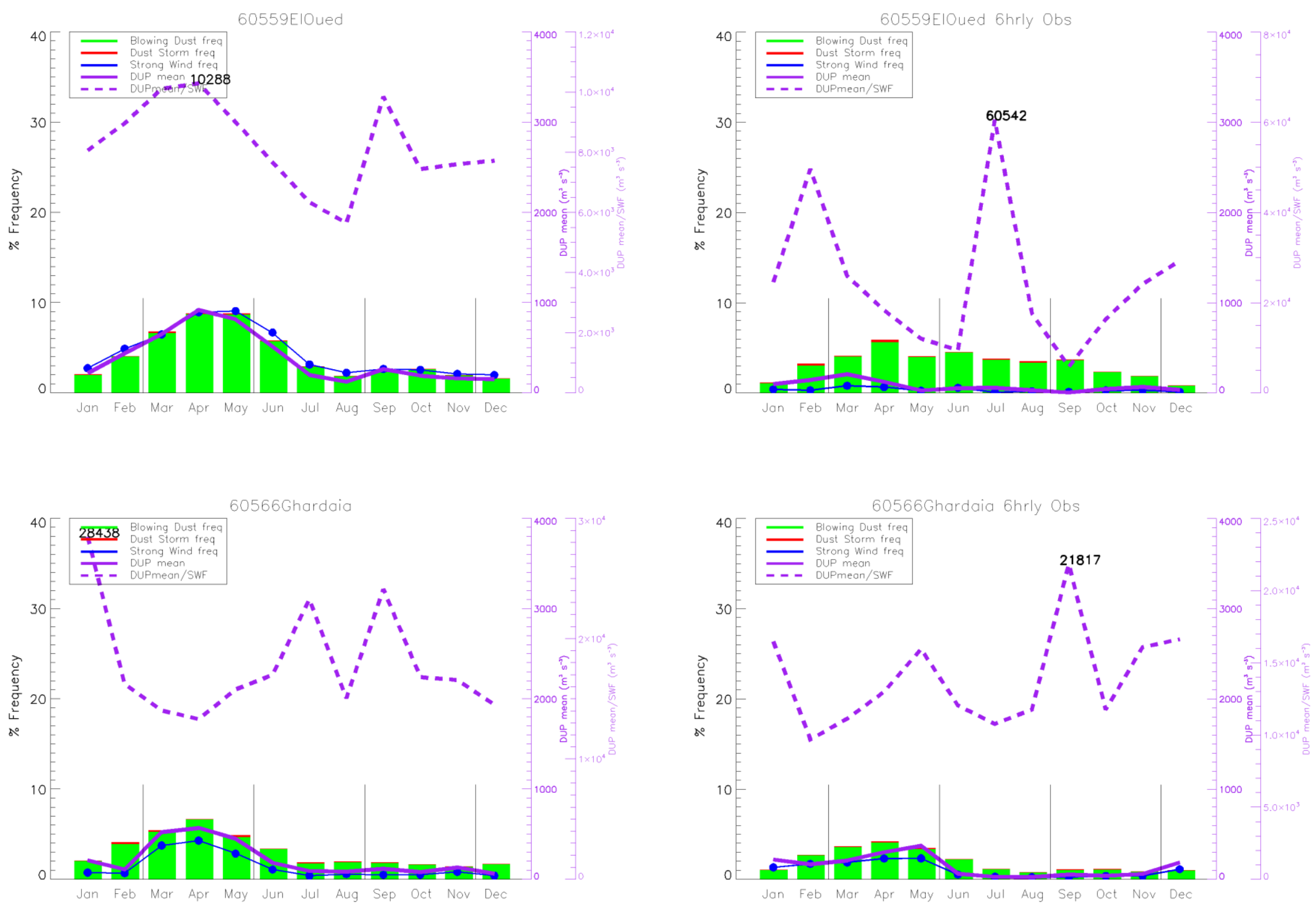

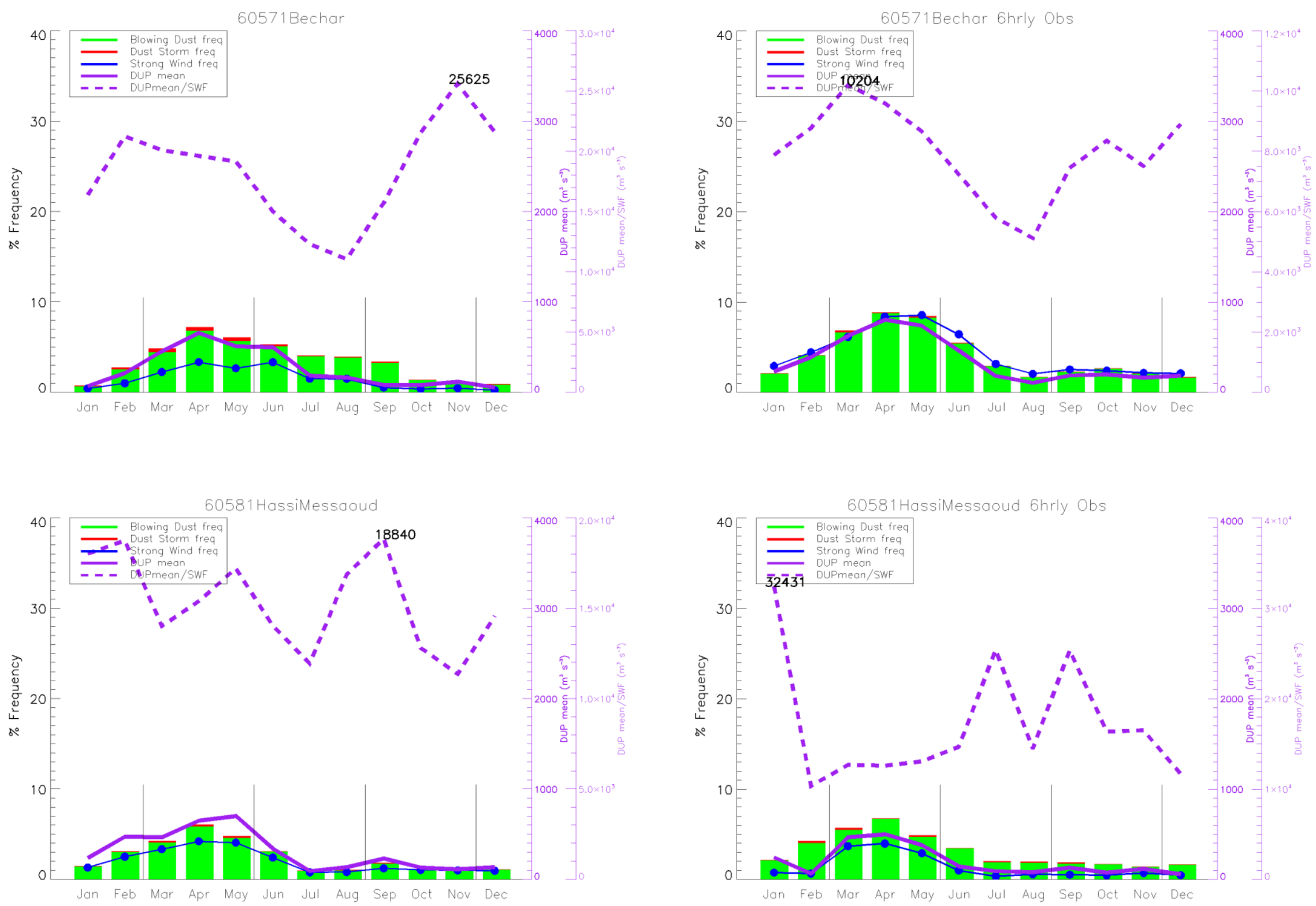

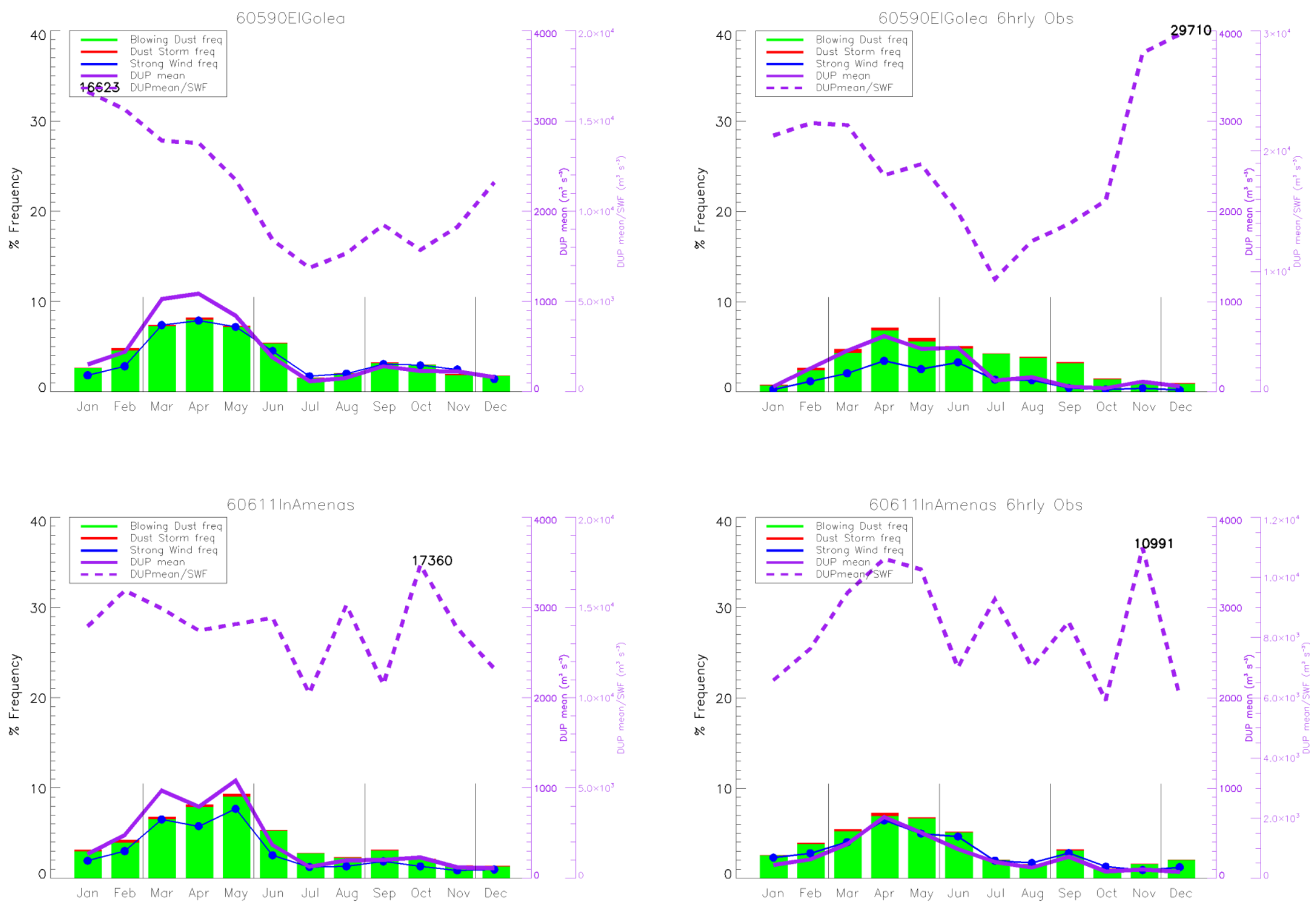

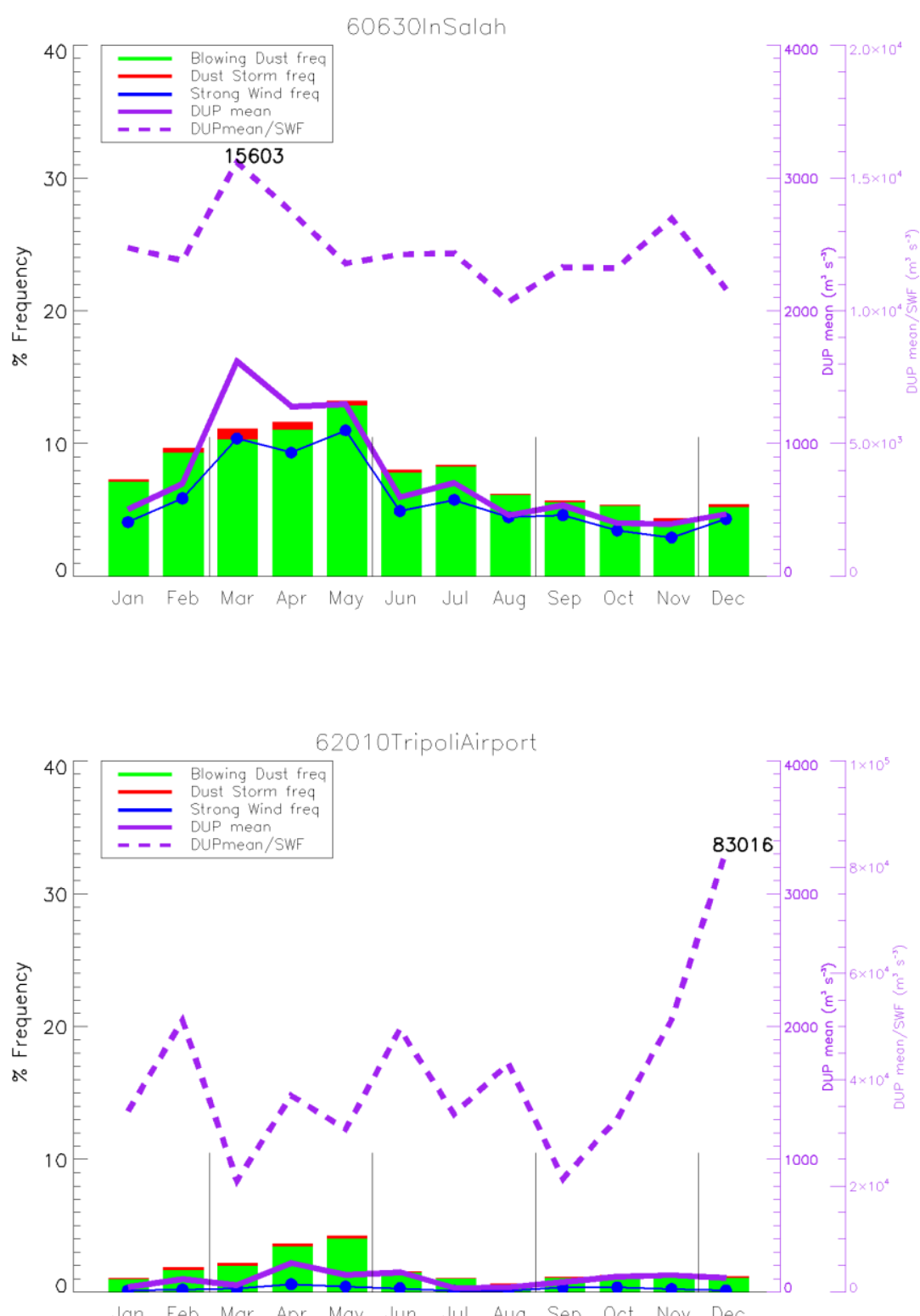
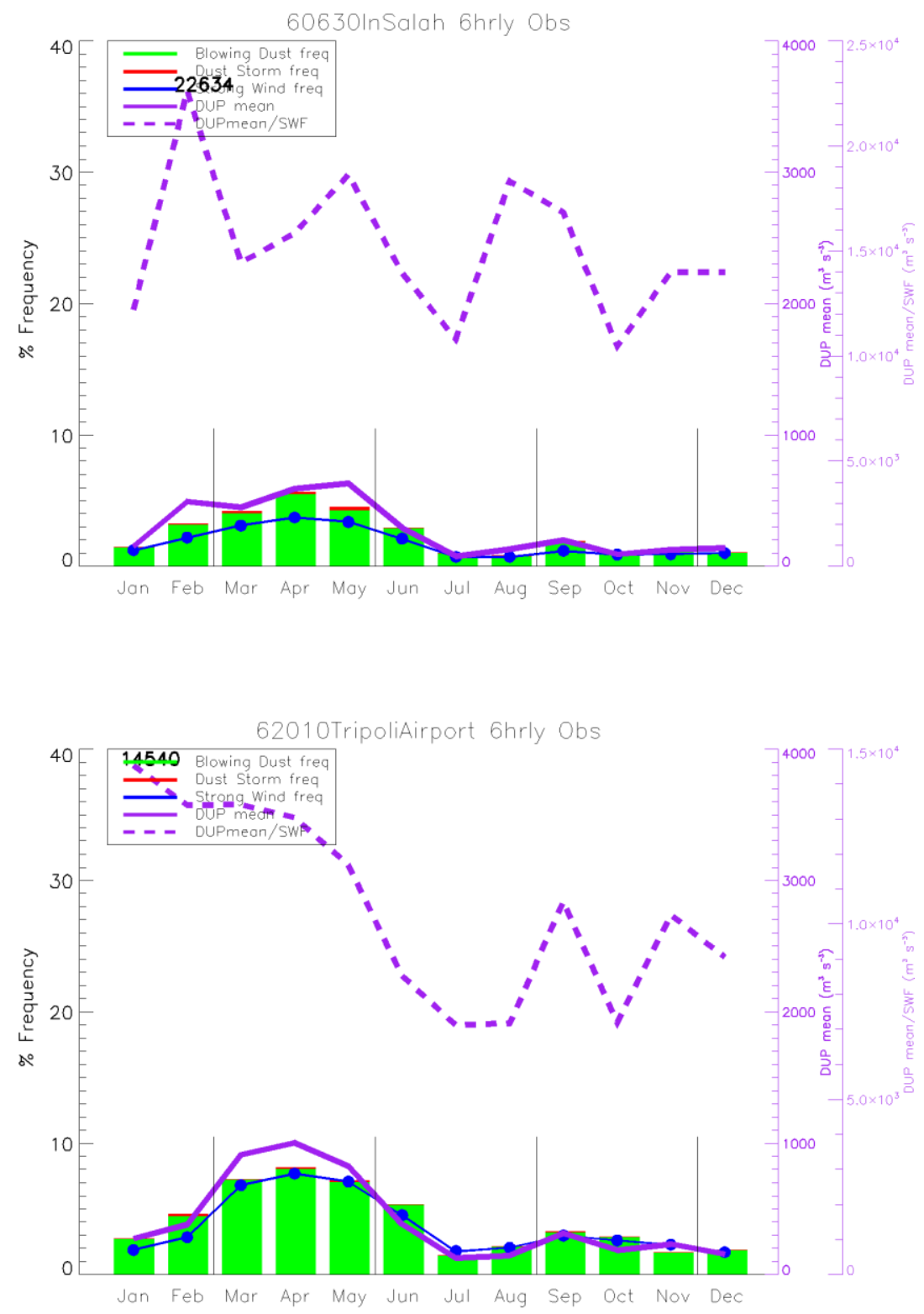

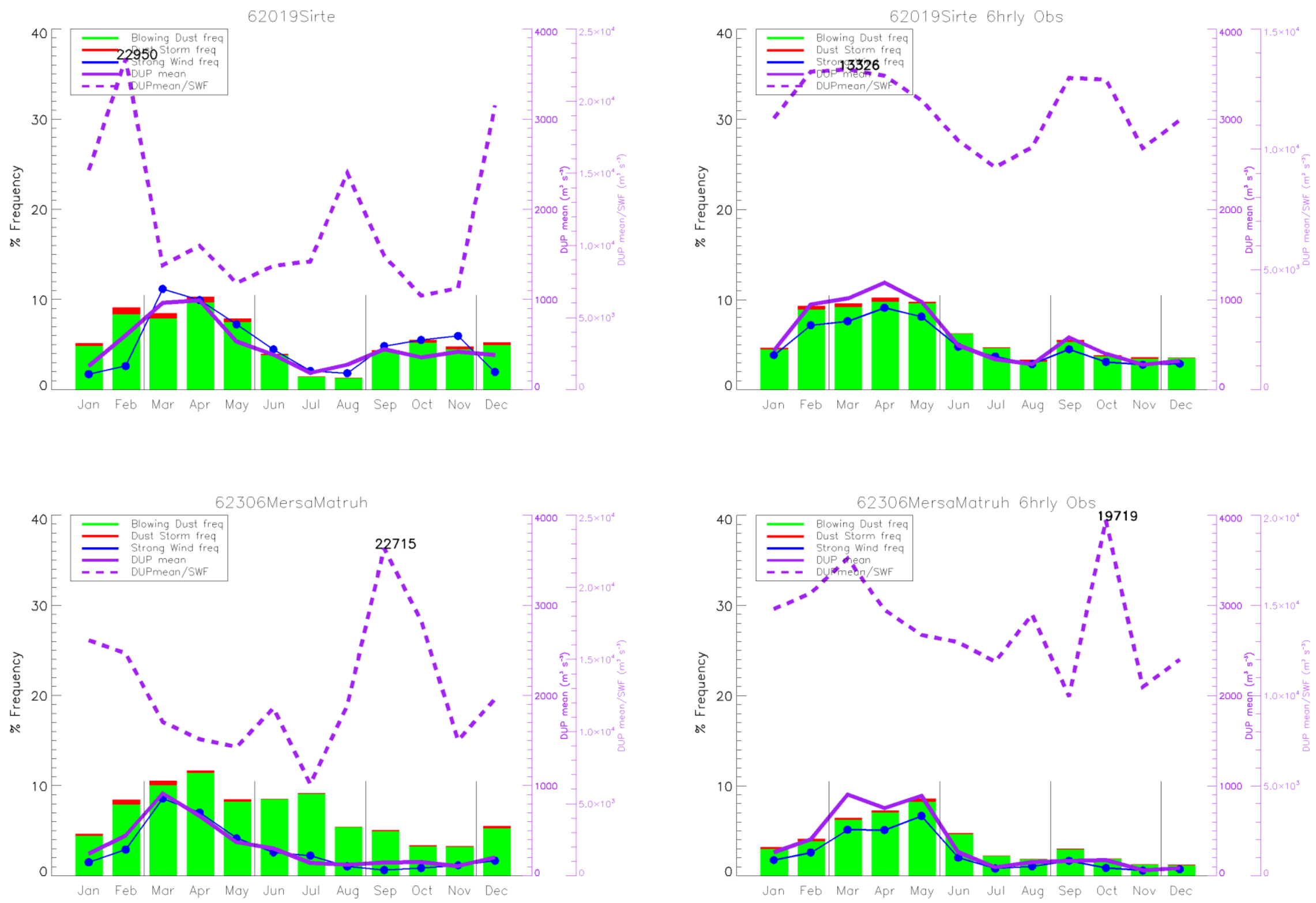

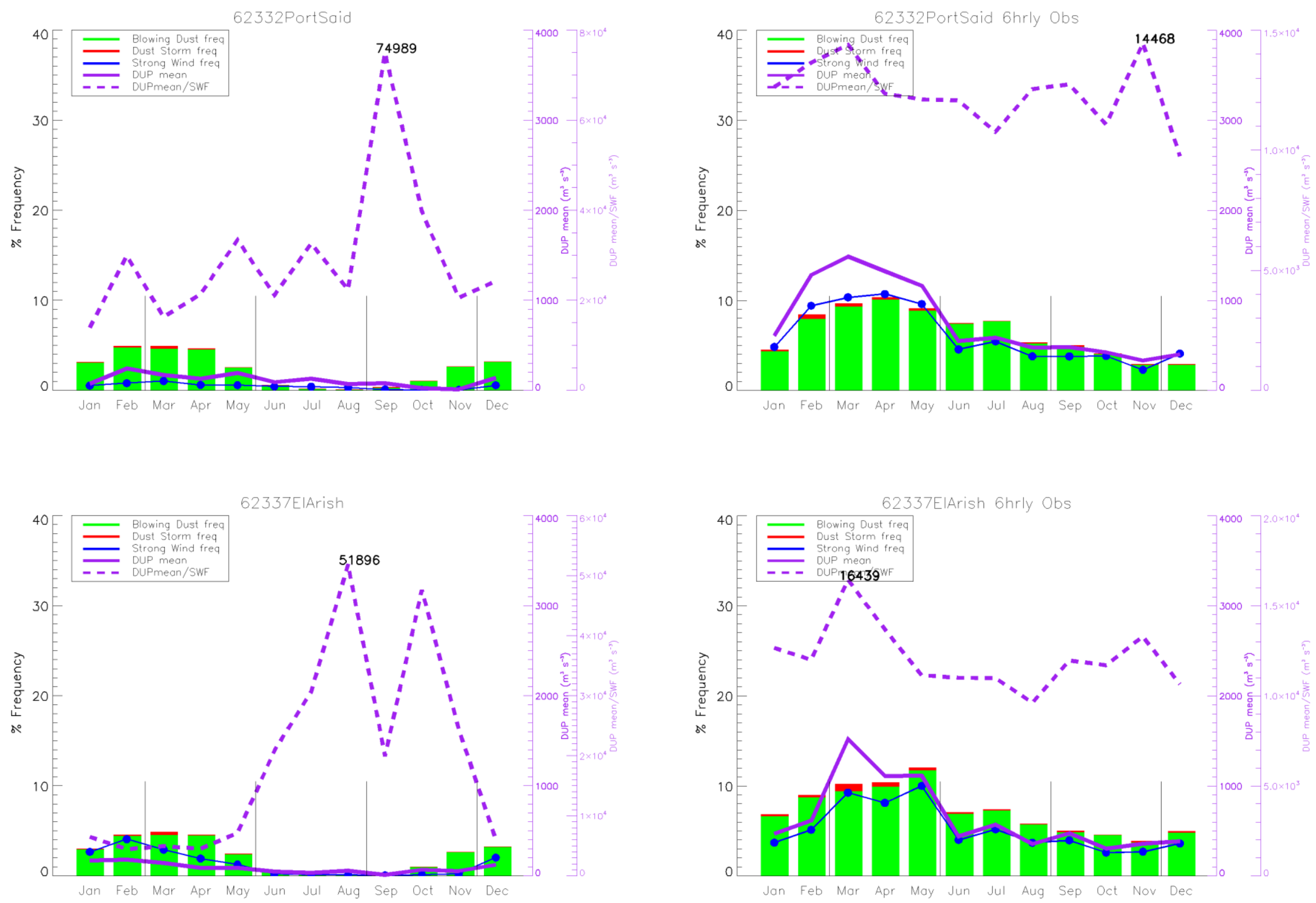

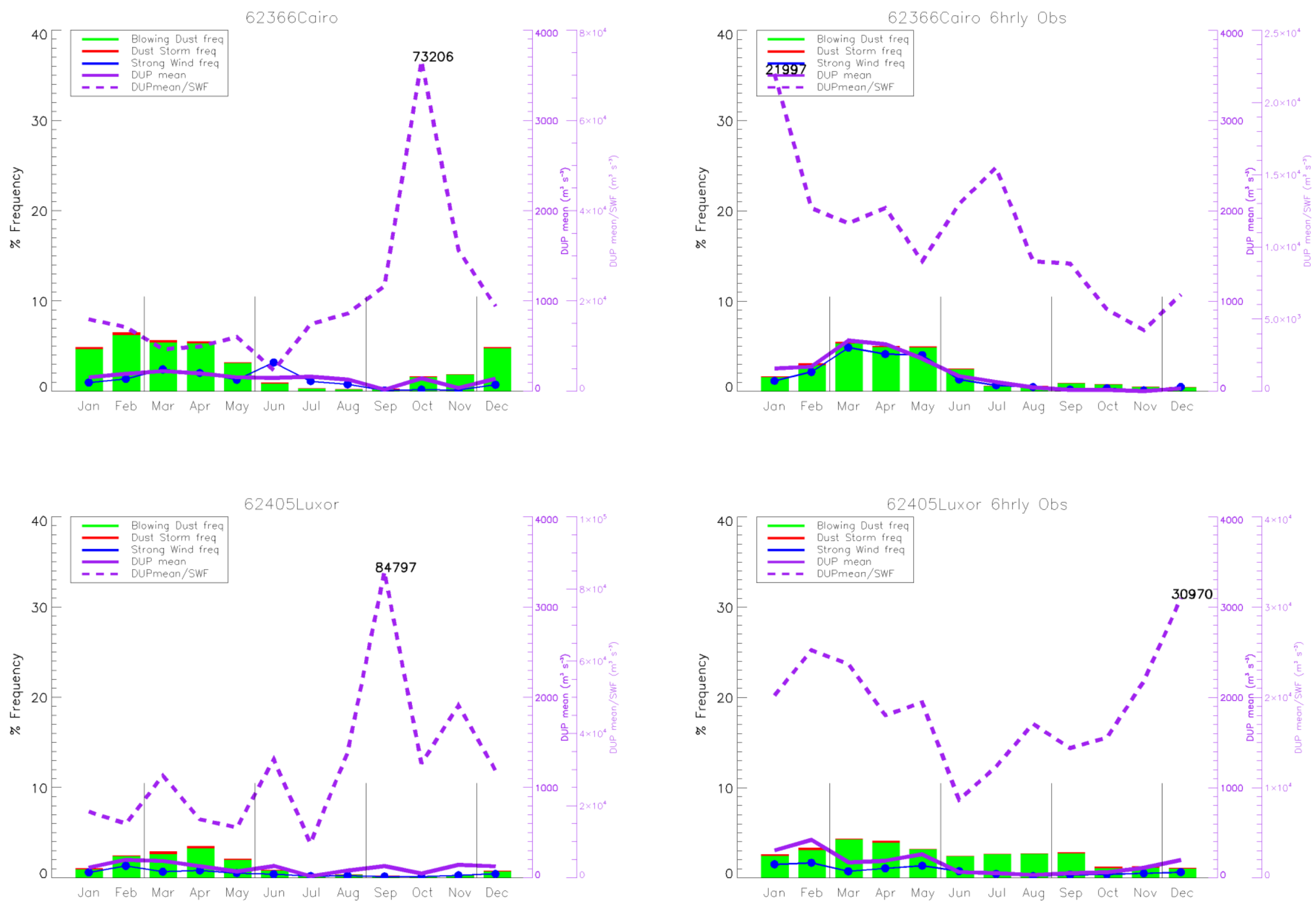

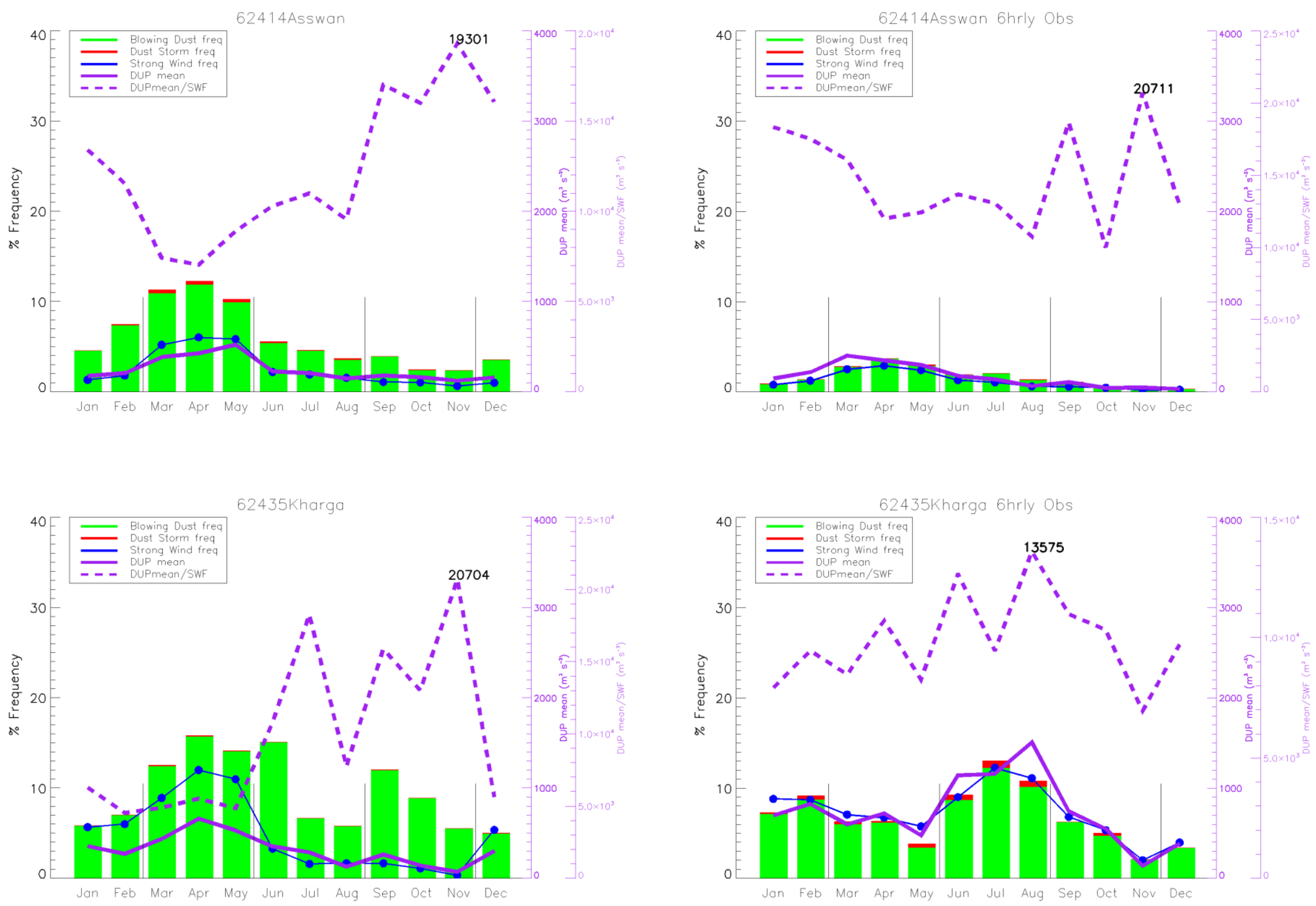
Figure S2. Mean seasonal cycle comparison using six hourly (right plots) and three hourly (left plots) observations for 18 stations which have stable three hourly reporting. Monthly frequency of FDE, split into BDF (green bars) and DSF (red bars), as well as SWF (blue line), DUP mean (solid purple) and DUP Intensity (DUP mean/SWF, dashed purple, maximum values given), all computed using the seasonally varying T50 (for definitions of parameters, see Sect. 2.2.1, main paper). Thin vertical black lines indicate seasonal boundaries where thresholds change. Note the different vertical axes for DUP intensity. 
 \\ 3000}

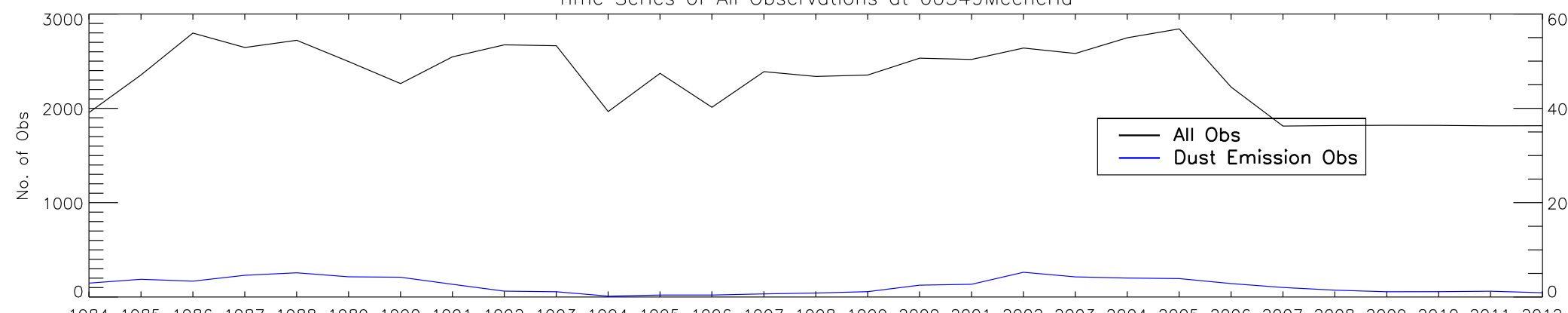

19841985198619871988198919901991199219931994199519961997199819992000200120022003200420052006200720082009201020112012 Time

Seasonal Time Series of All Observations at 60549Mecheria

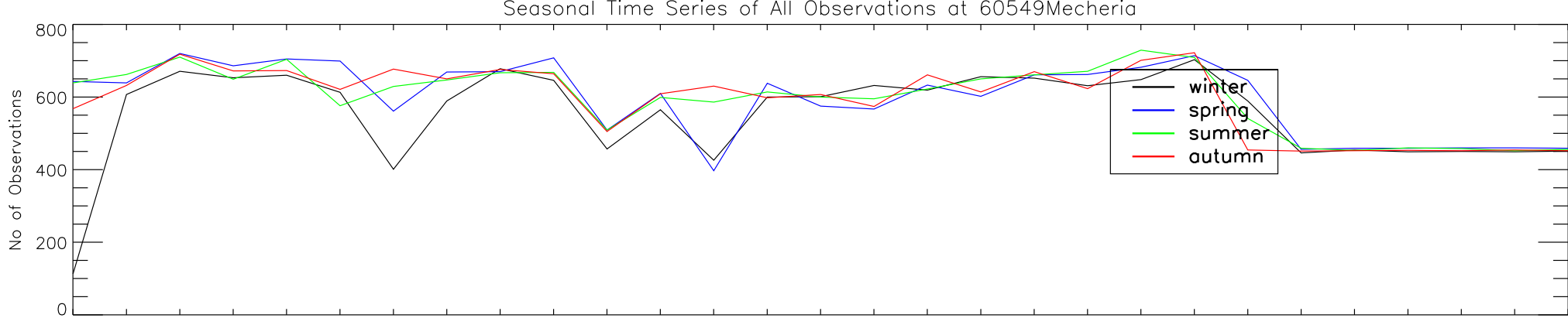

19841985198619871988198919901991199219931994199519961997199819992000200120022003200420052006200720082009201020112012

Seasonal Time Series of Dust Observations at 60549Mecheria

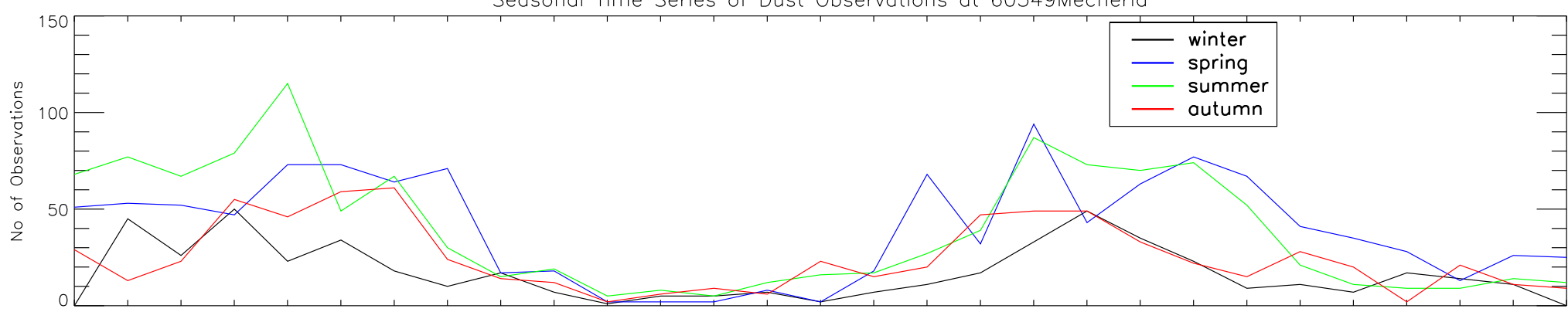

19841985198619871988198919901991199219931994199519961997199819992000200120022003200420052006200720082009201020112012 Time 


\section{0}

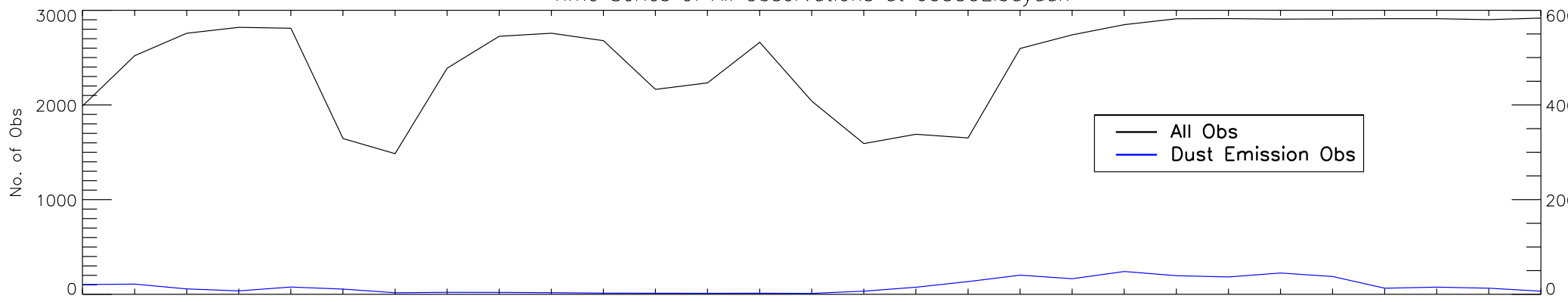

19841985198619871988198919901991199219931994199519961997199819992000200120022003200420052006200720082009201020112012 Time

Seasonal Time Series of All Observations at 60550Elbayadh

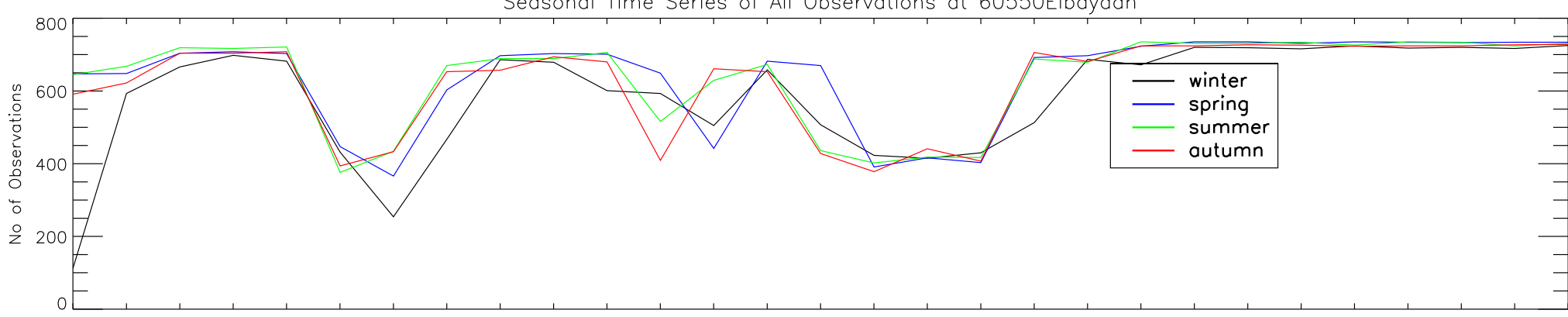

19841985198619871988198919901991199219931994199519961997199819992000200120022003200420052006200720082009201020112012

Seasonal Time Series of Dust Observations at 60550Elbayadh

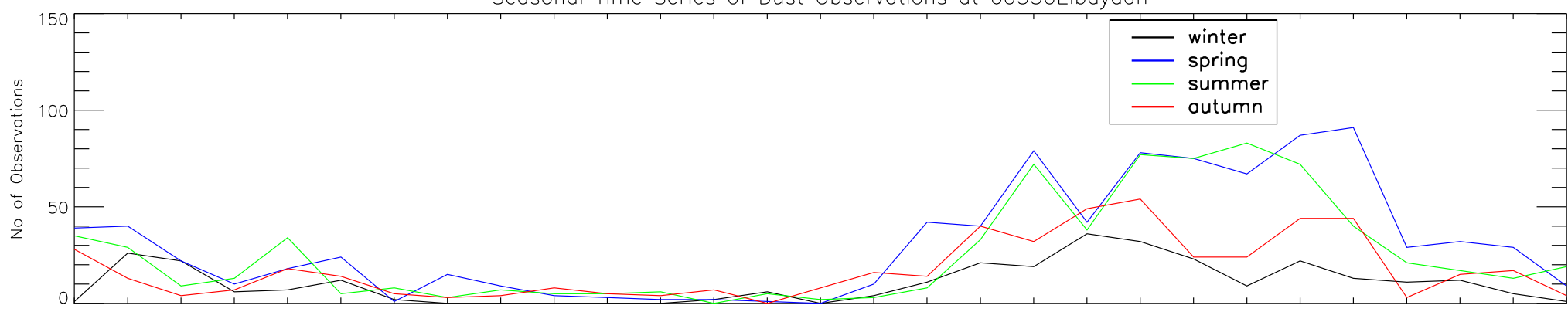

19841985198619871988198919901991199219931994199519961997199819992000200120022003200420052006200720082009201020112012 Time 


\section{0}

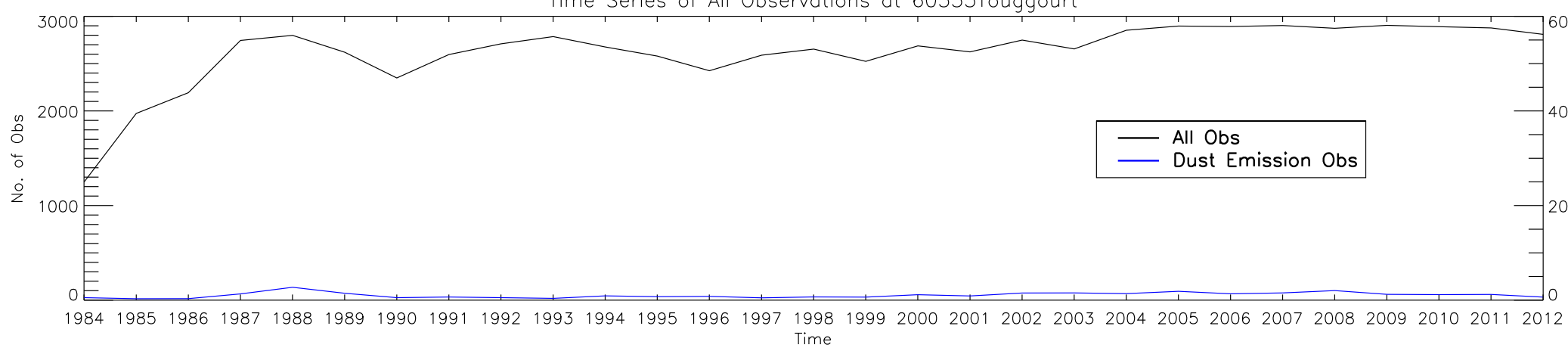

Seasonal Time Series of All Observations at 60555Touggourt

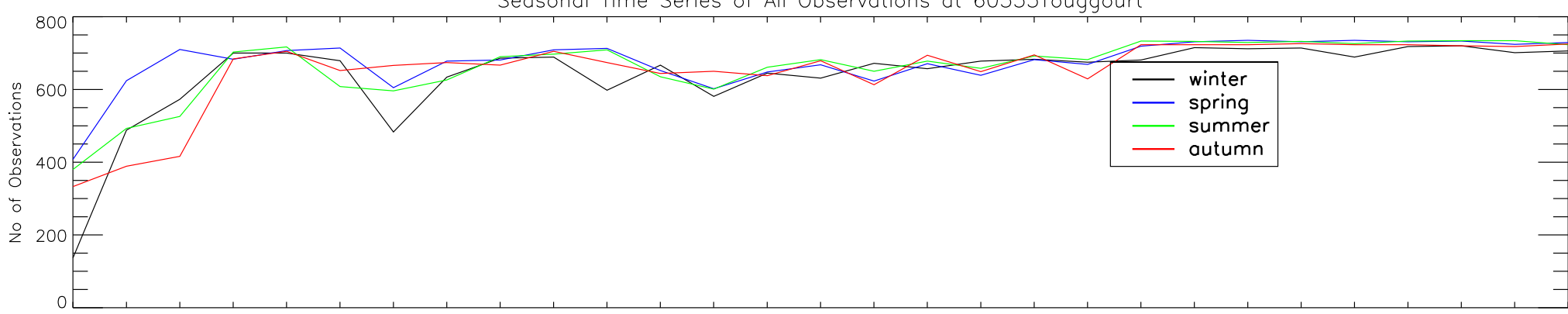

19841985198619871988198919901991199219931994199519961997199819992000200120022003200420052006200720082009201020112012

Seasonal Time Series of Dust Observations at 60555Touggourt

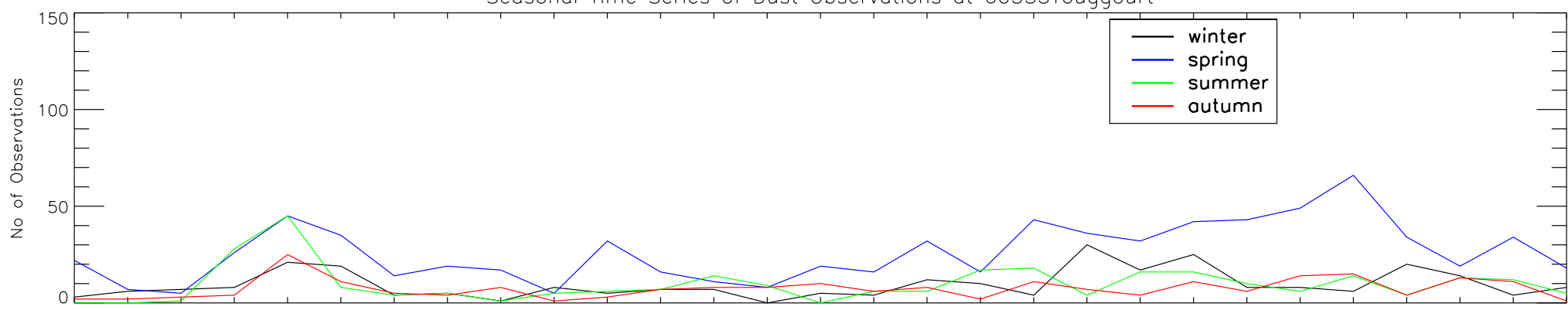

19841985198619871988198919901991199219931994199519961997199819992000200120022003200420052006200720082009201020112012 Time 
Time Series of All Observations at 60559EIOued

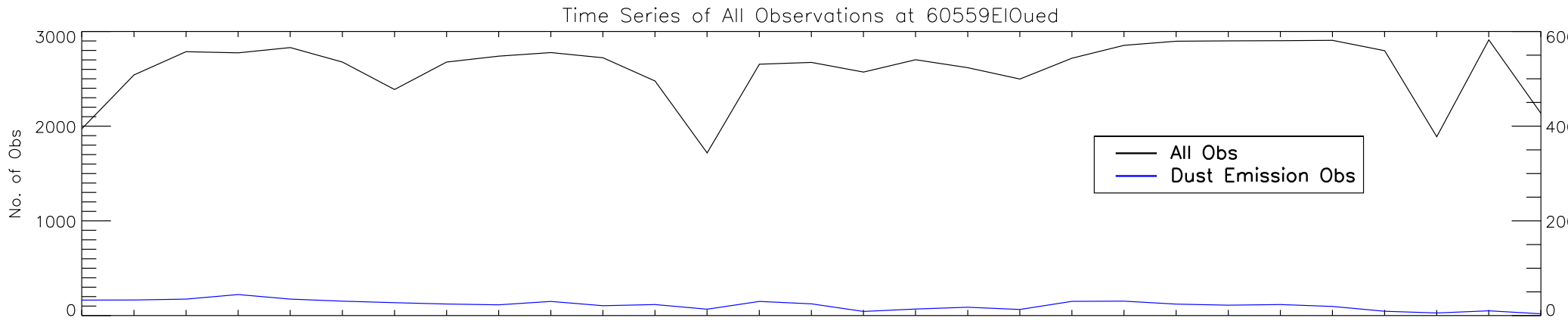

19841985198619871988198919901991199219931994199519961997199819992000200120022003200420052006200720082009201020112012 Time

Seasonal Time Series of All Observations at 60559ElOued

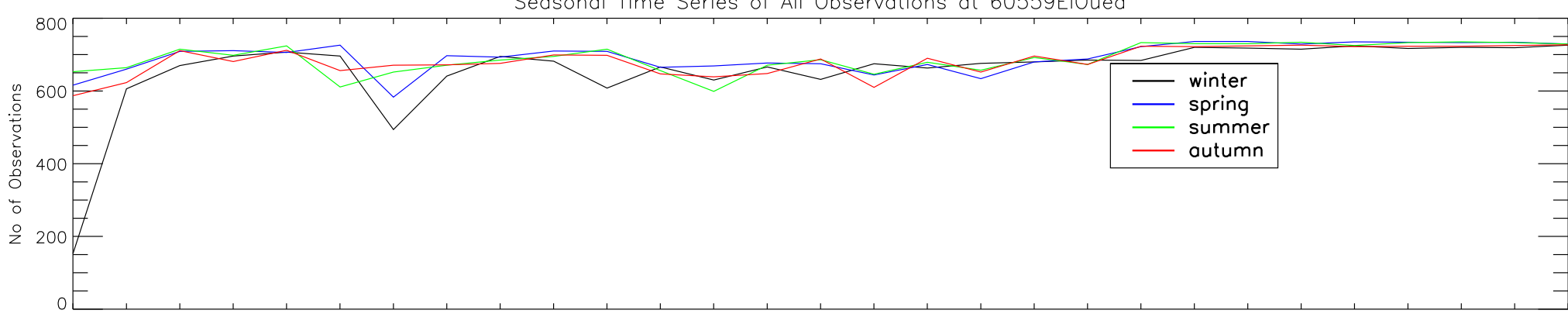

19841985198619871988198919901991199219931994199519961997199819992000200120022003200420052006200720082009201020112012

Seasonal Time Series of Dust Observations at 60559EIOued

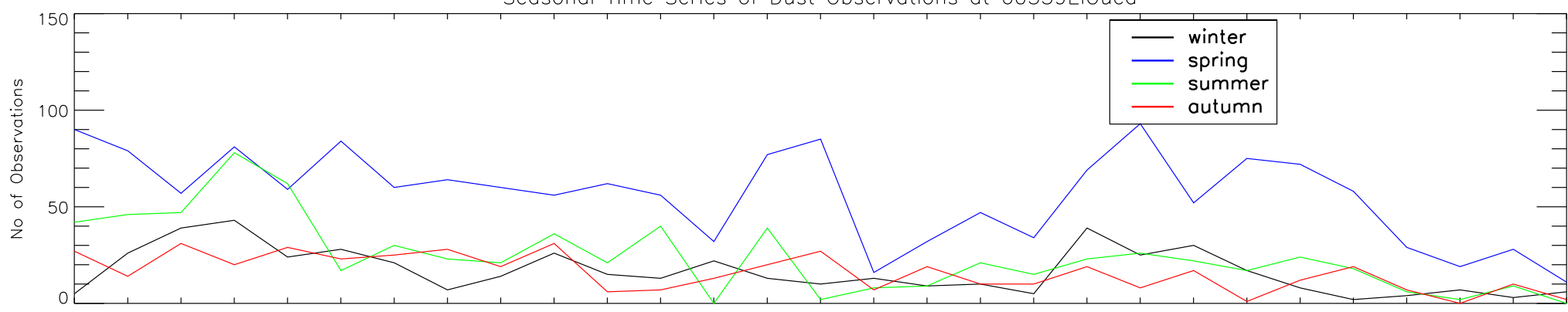

19841985198619871988198919901991199219931994199519961997199819992000200120022003200420052006200720082009201020112012 Time 
 \\ 3000}

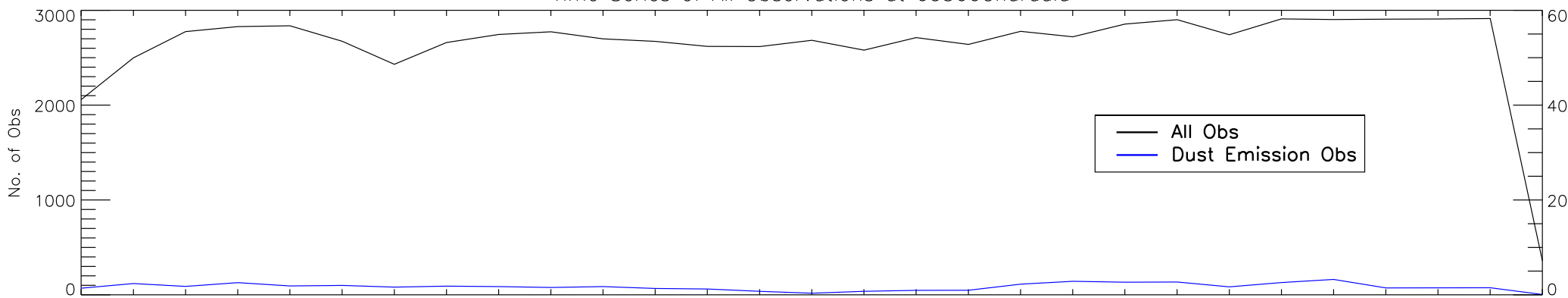

19841985198619871988198919901991199219931994199519961997199819992000200120022003200420052006200720082009201020112012 Time

Seasonal Time Series of All Observations at 60566Ghardaia

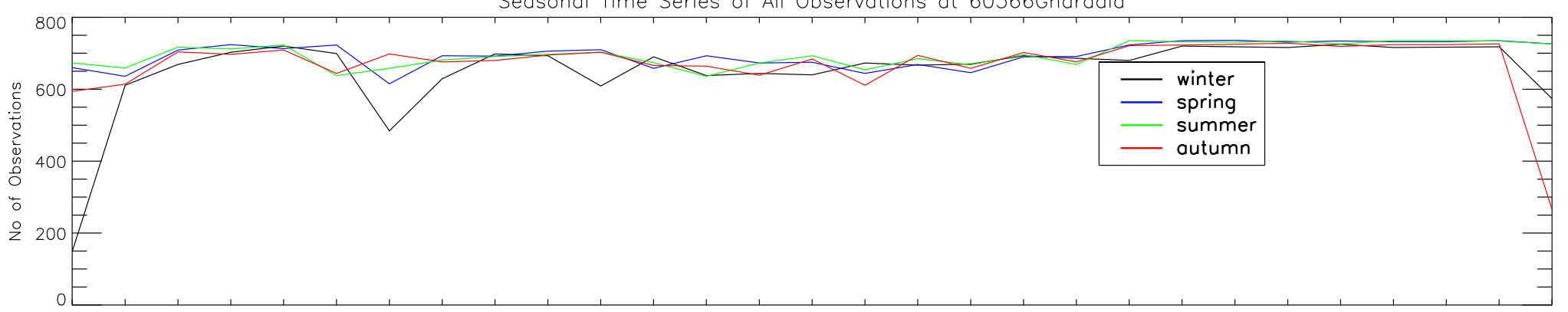

19841985198619871988198919901991199219931994199519961997199819992000200120022003200420052006200720082009201020112012

Seasonal Time Series of Dust Observations at 60566Ghardaia

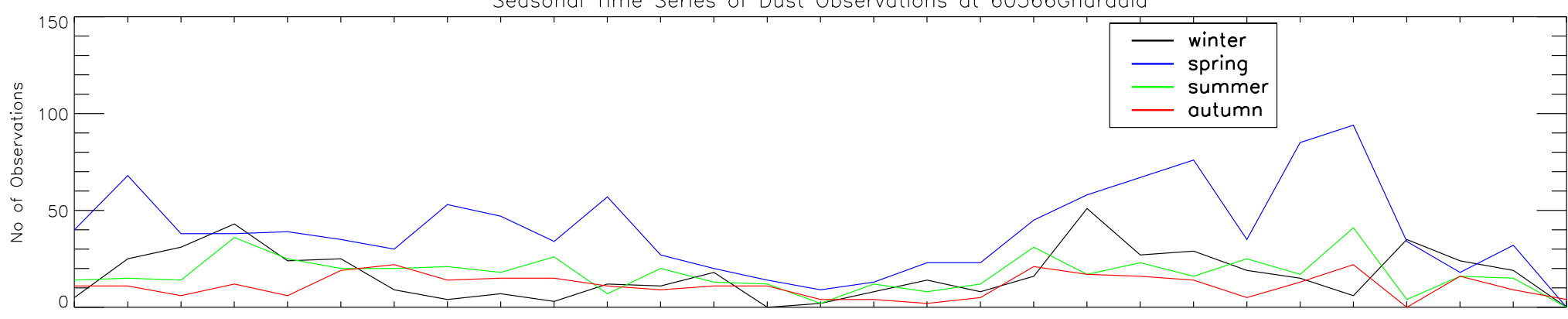

19841985198619871988198919901991199219931994199519961997199819992000200120022003200420052006200720082009201020112012 Time 
 \\ $3000 F_{1}$}

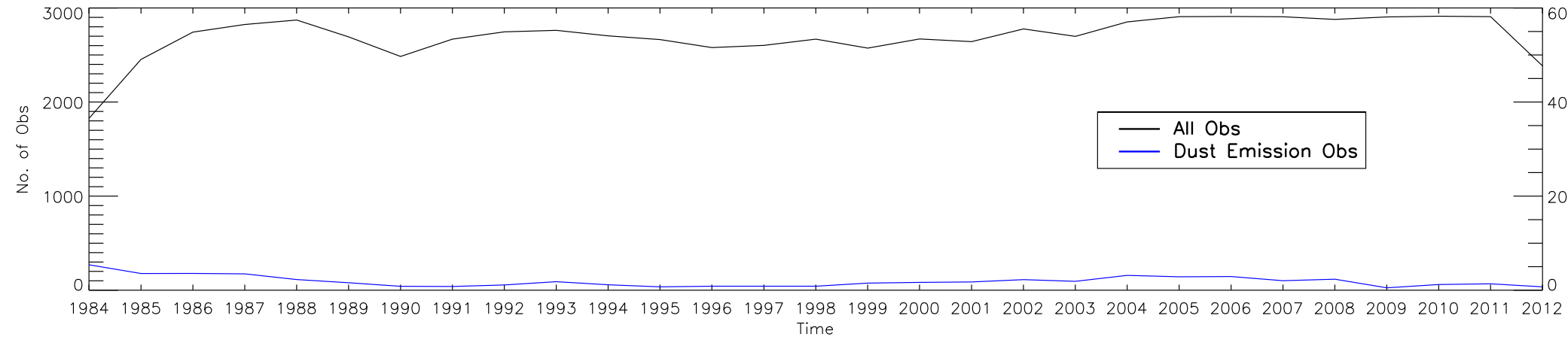

Seasonal Time Series of All Observations at 60571Bechar

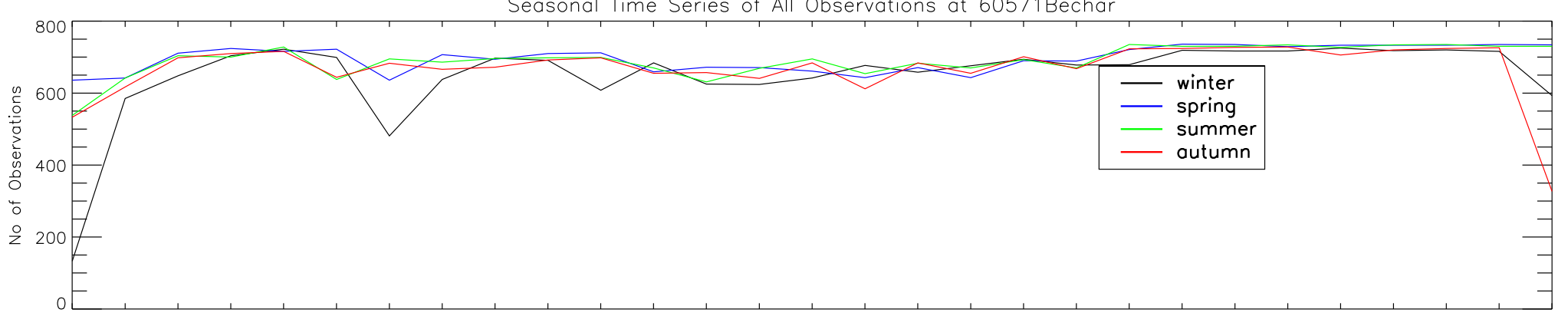

19841985198619871988198919901991199219931994199519961997199819992000200120022003200420052006200720082009201020112012

Seasonal Time Series of Dust Observations at 60571Bechar

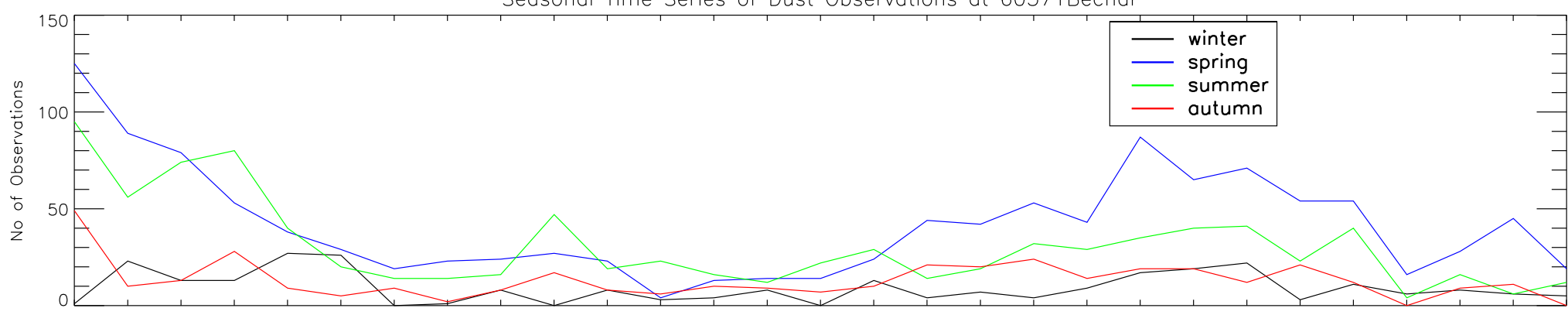

19841985198619871988198919901991199219931994199519961997199819992000200120022003200420052006200720082009201020112012 Time 
Time Series of All Observations at 605800uargla

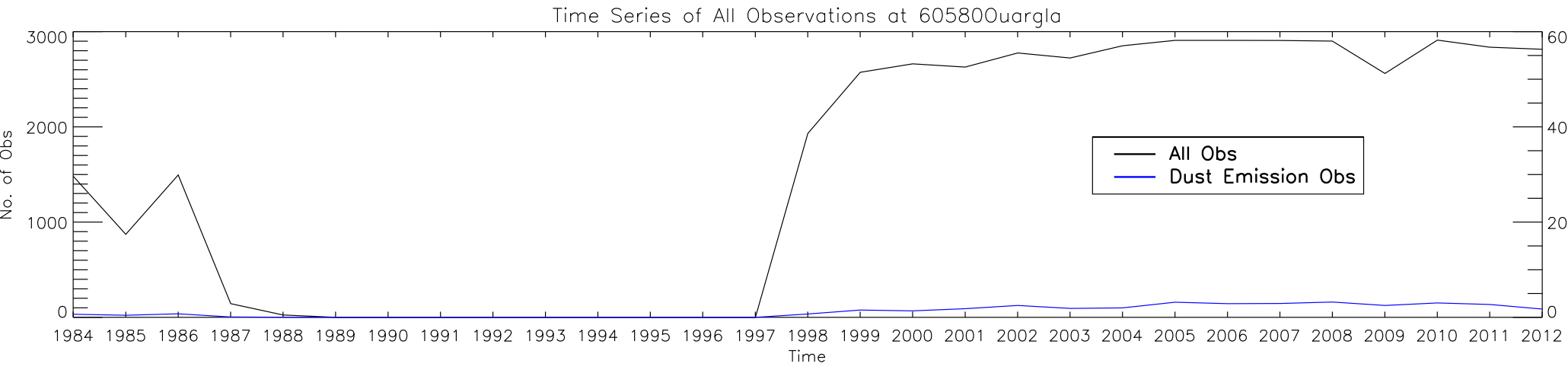

Seasonal Time Series of All Observations at 605800uargla

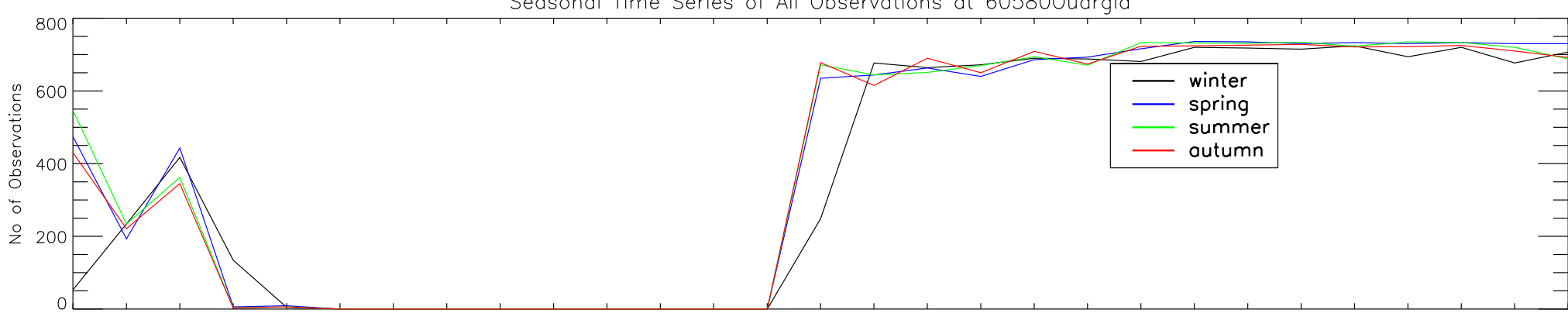

19841985198619871988198919901991199219931994199519961997199819992000200120022003200420052006200720082009201020112012

Seasonal Time Series of Dust Observations at 605800uargla

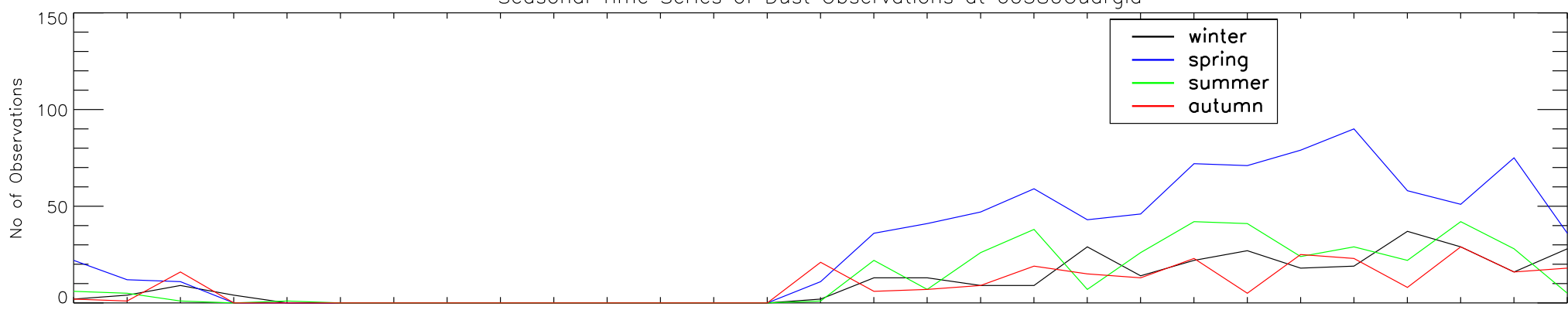

19841985198619871988198919901991199219931994199519961997199819992000200120022003200420052006200720082009201020112012 Time 
 \\ Time Series of All Observations at 60581 HassiMessaoud}

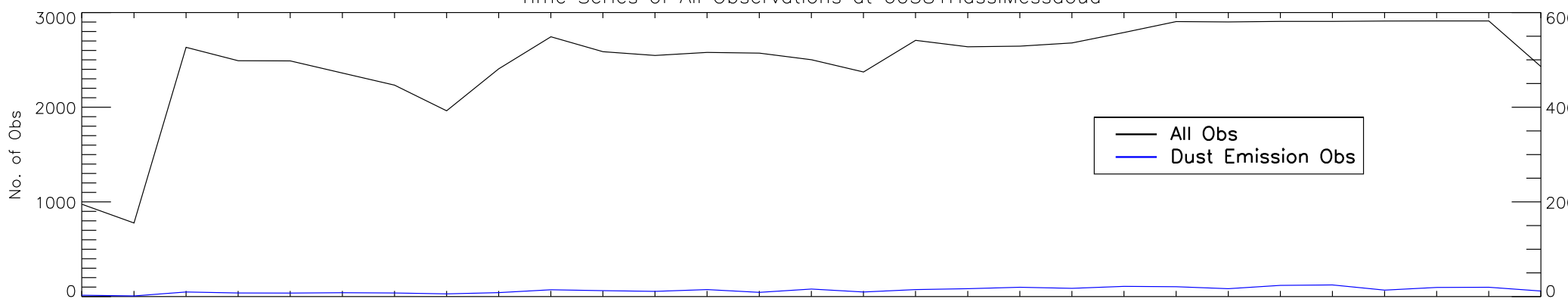

19841985198619871988198919901991199219931994199519961997199819992000200120022003200420052006200720082009201020112012 Time

Seasonal Time Series of All Observations at 60581HassiMessaoud

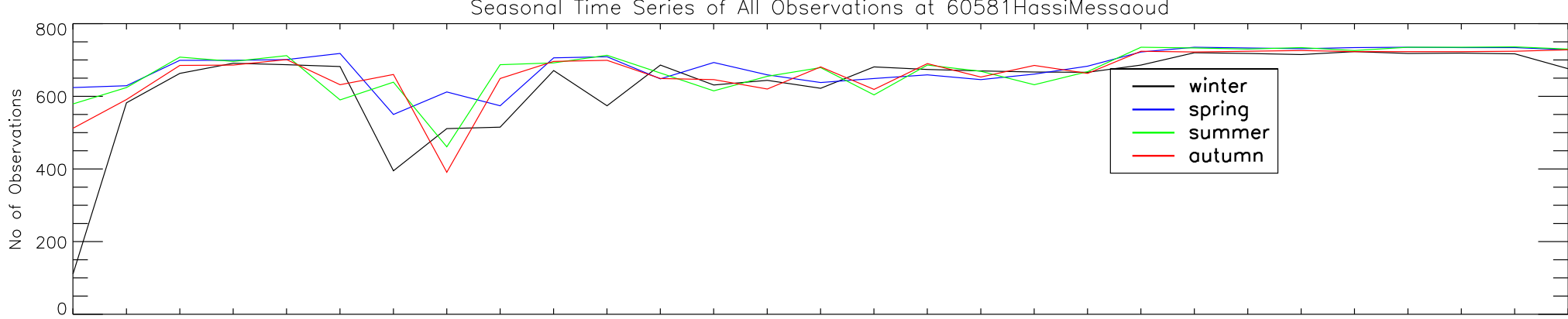

19841985198619871988198919901991199219931994199519961997199819992000200120022003200420052006200720082009201020112012

Seasonal Time Series of Dust Observations at 60581 HassiMessaoud

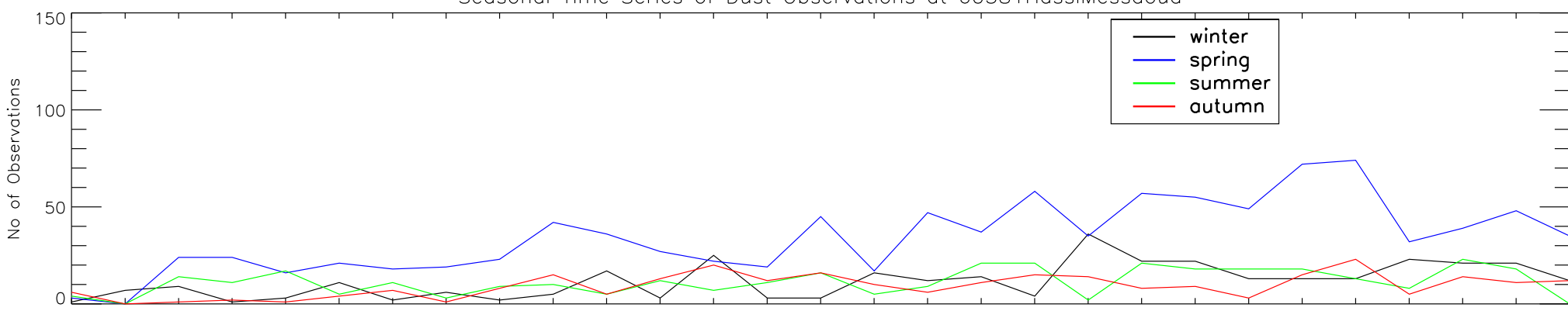

19841985198619871988198919901991199219931994199519961997199819992000200120022003200420052006200720082009201020112012 Time 
 \\ 3000}

\section{All Obs}

Dust Emission Obs

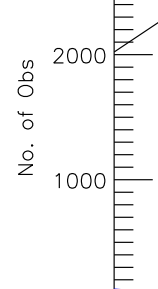

19841985198619871988198919901991199219931994199519961997199819992000200120022003200420052006200720082009201020112012 Time

Seasonal Time Series of All Observations at 60590EIGolea

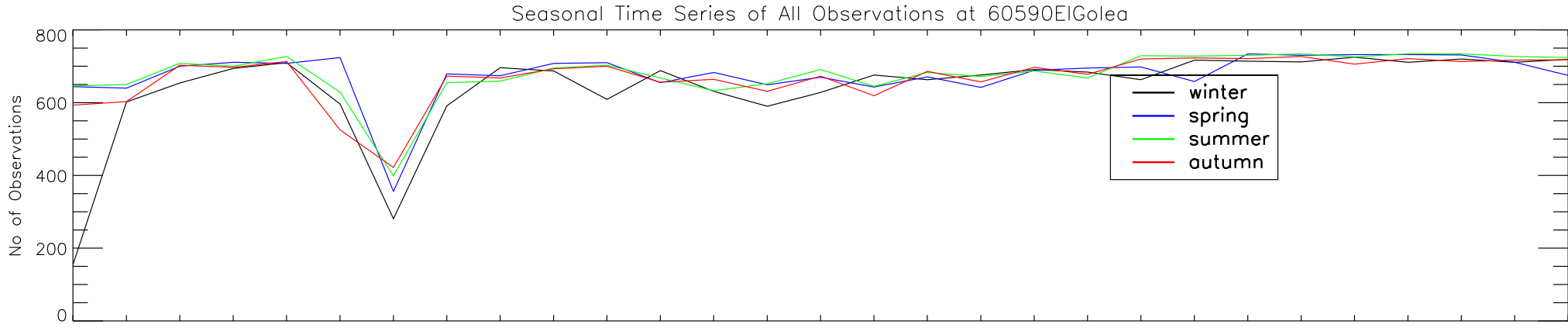

19841985198619871988198919901991199219931994199519961997199819992000200120022003200420052006200720082009201020112012

Seasonal Time Series of Dust Observations at 60590EIGolea

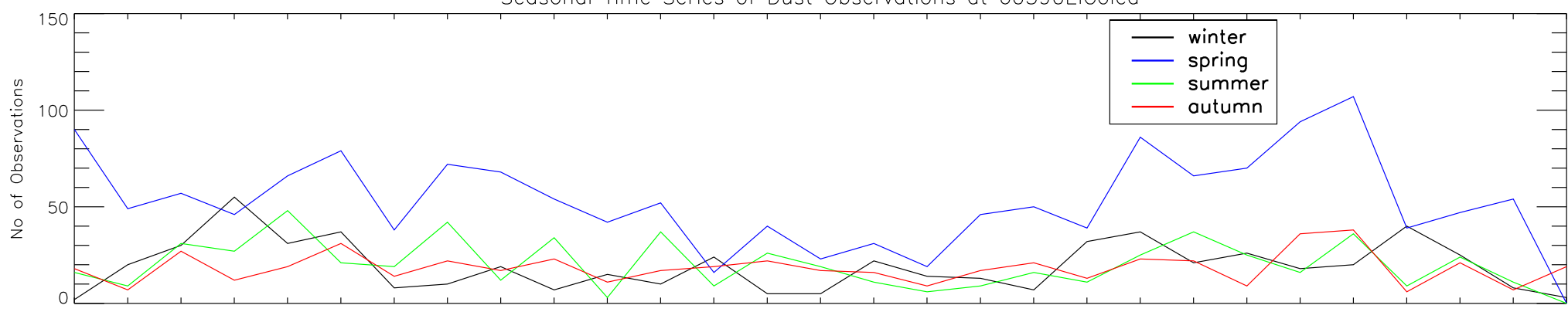

19841985198619871988198919901991199219931994199519961997199819992000200120022003200420052006200720082009201020112012 Time 
 \\ 3000}

\section{All Obs}

Dust Emission Obs

$\dot{0}$

$1984198519861987198819891990199119921993 \quad 1994199519961997$ Time

Seasonal Time Series of All Observations at 60611 InAmenas

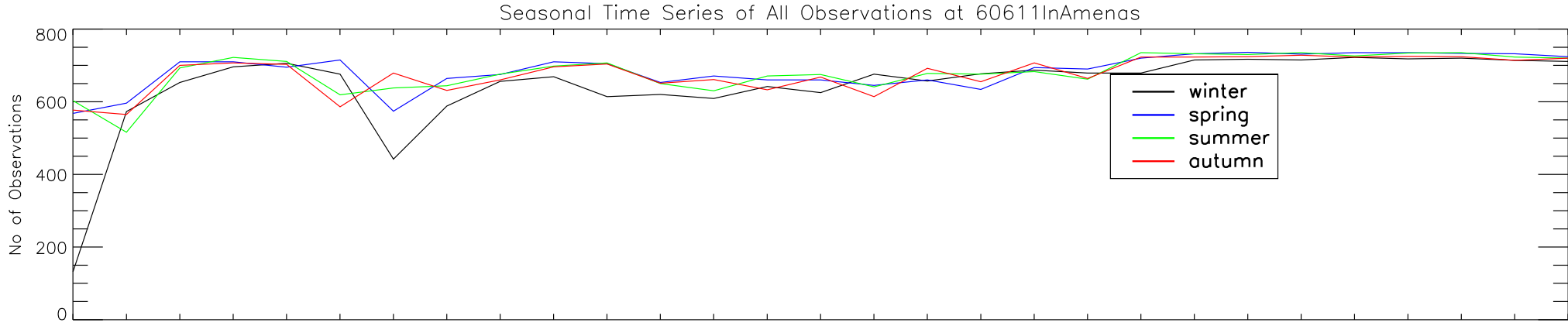

19841985198619871988198919901991199219931994199519961997199819992000200120022003200420052006200720082009201020112012

Seasonal Time Series of Dust Observations at 60611 InAmenas

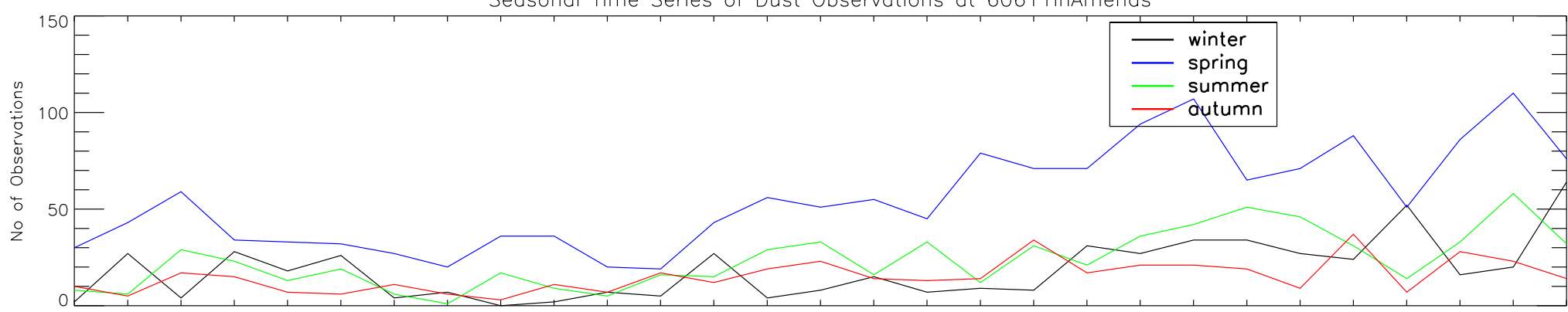

19841985198619871988198919901991199219931994199519961997199819992000200120022003200420052006200720082009201020112012 Time 
 \\ $3000-T_{1}$ Time Series of All Observations at 60620Adrar}

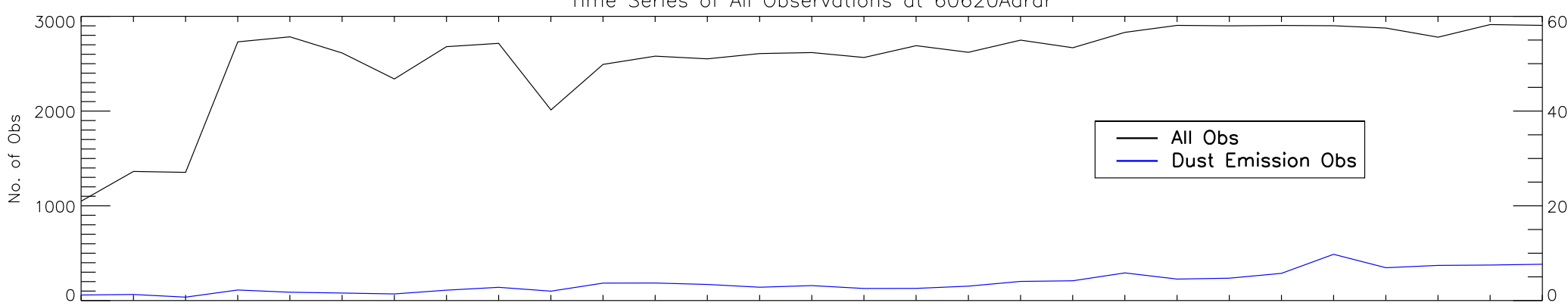

19841985198619871988198919901991199219931994199519961997199819992000200120022003200420052006200720082009201020112012 Time

Seasonal Time Series of All Observations at 60620Adrar

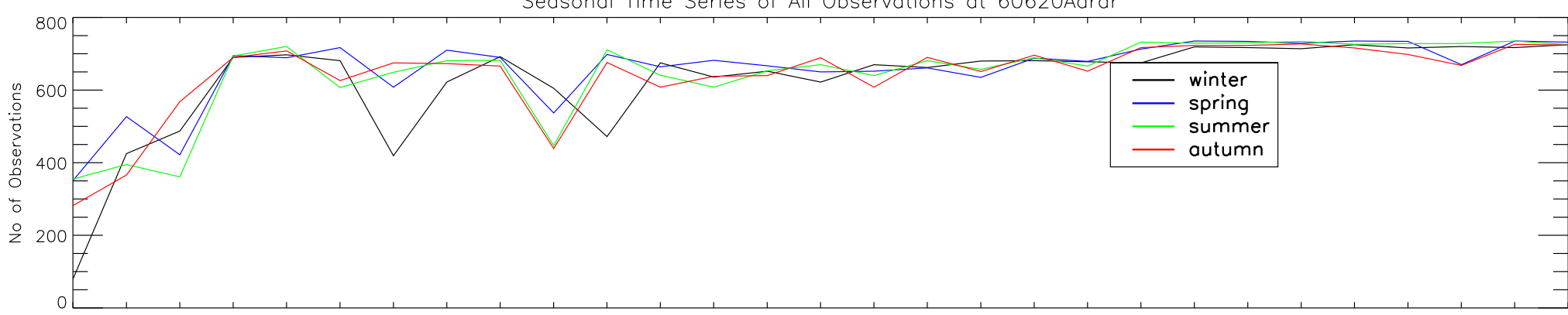

19841985198619871988198919901991199219931994199519961997199819992000200120022003200420052006200720082009201020112012

Seasonal Time Series of Dust Observations at 60620Adrar

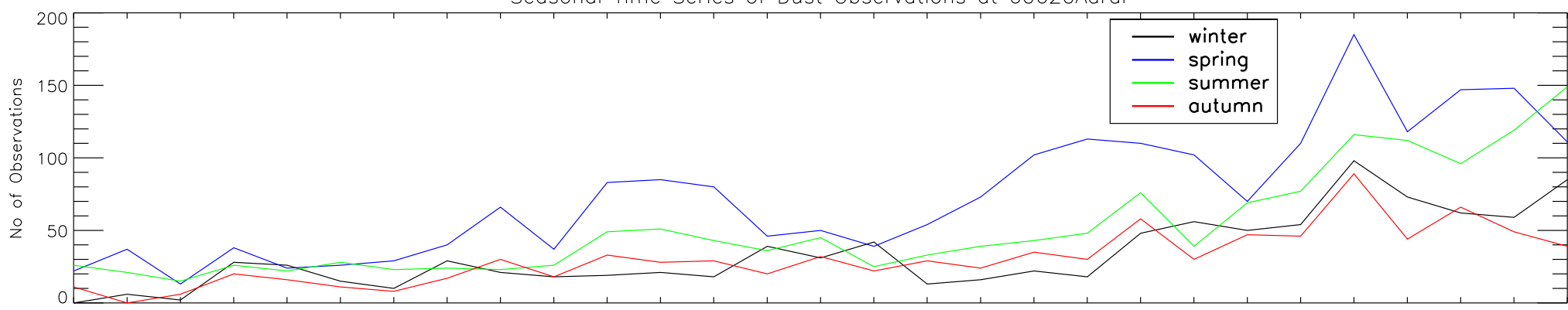

19841985198619871988198919901991199219931994199519961997199819992000200120022003200420052006200720082009201020112012 Time 
 \\ $3000+S_{1}$ Time Series of All Observations at 60630InSalah}

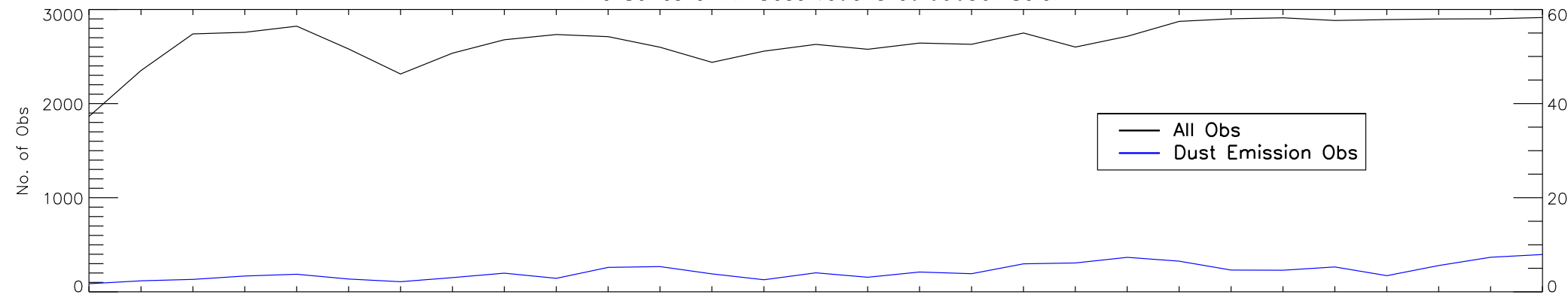

19841985198619871988198919901991199219931994199519961997199819992000200120022003200420052006200720082009201020112012 Time

Seasonal Time Series of All Observations at 60630 InSalah

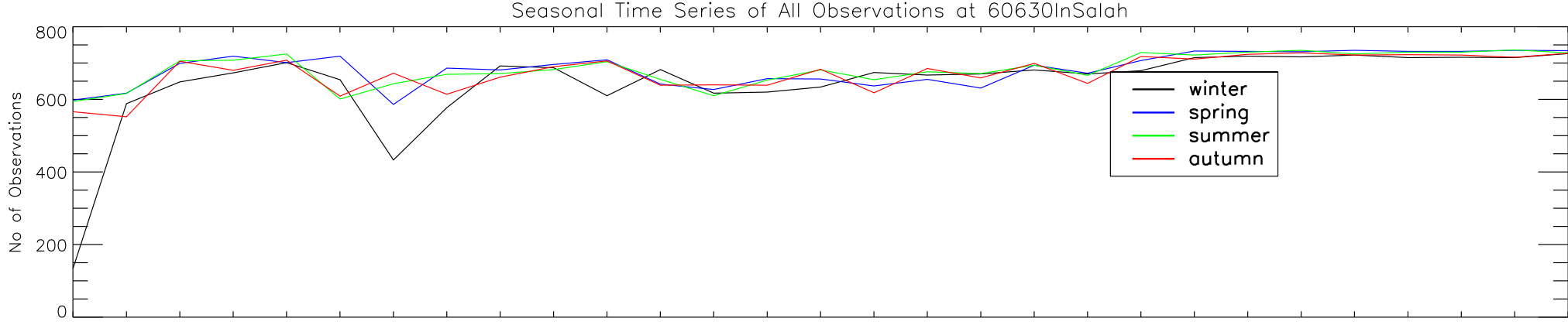

19841985198619871988198919901991199219931994199519961997199819992000200120022003200420052006200720082009201020112012

Seasonal Time Series of Dust Observations at 60630InSalah

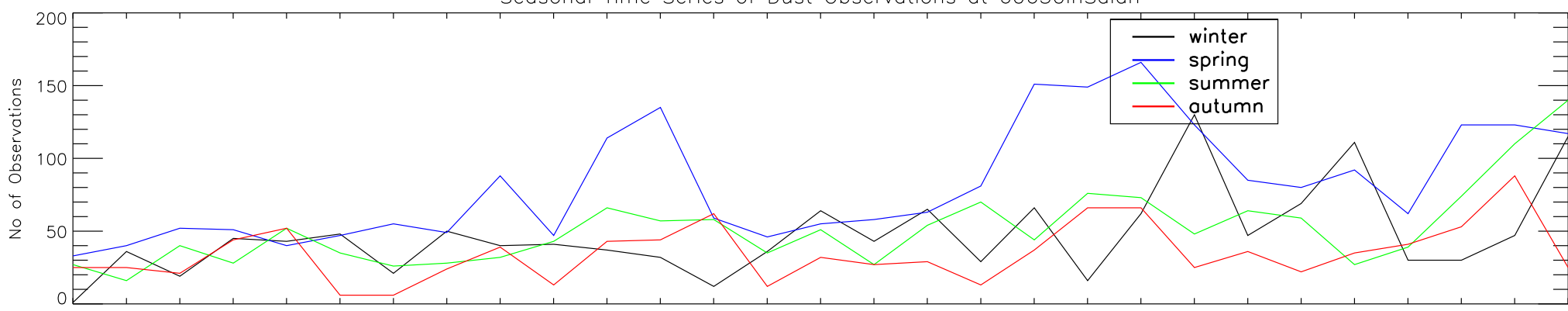

19841985198619871988198919901991199219931994199519961997199819992000200120022003200420052006200720082009201020112012 Time 
 \\ 3000}

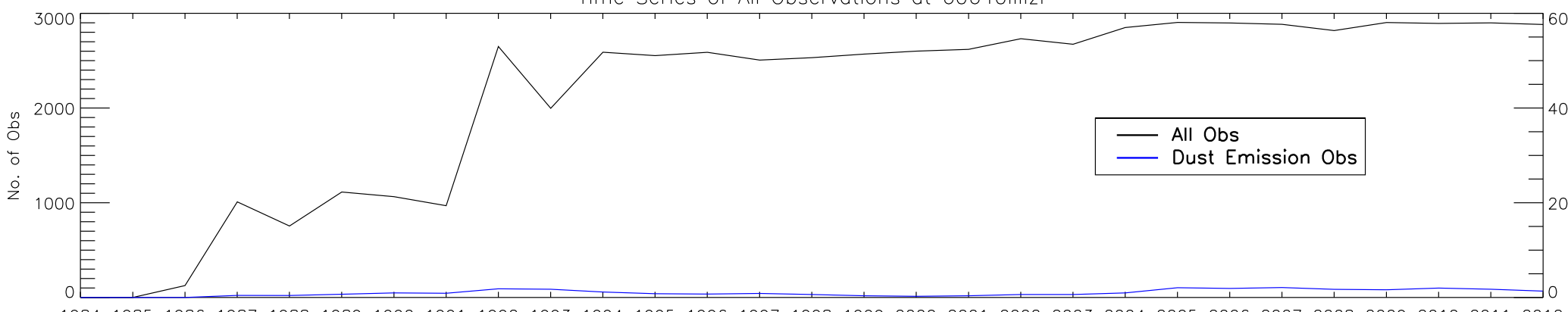

19841985198619871988198919901991199219931994199519961997199819992000200120022003200420052006200720082009201020112012 Time

Seasonal Time Series of All Observations at 60640 ollizi

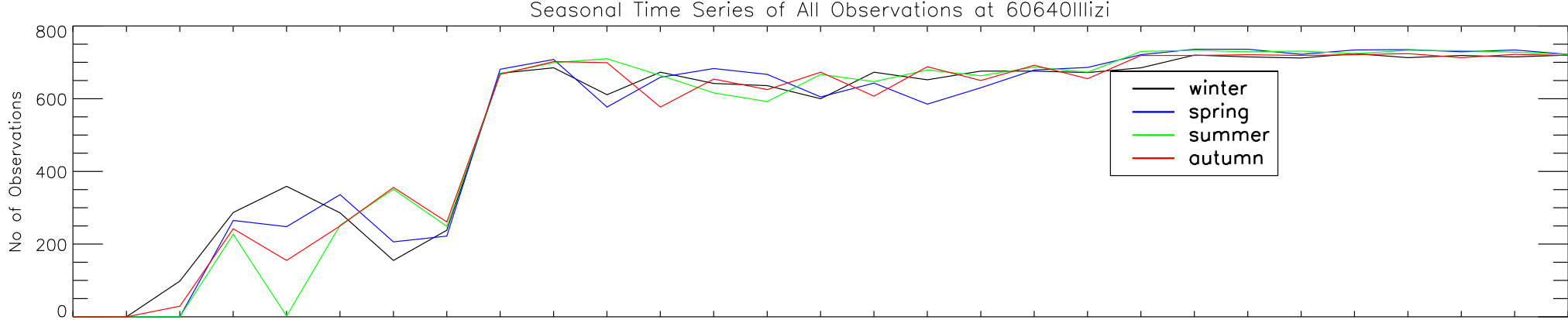

19841985198619871988198919901991199219931994199519961997199819992000200120022003200420052006200720082009201020112012

Seasonal Time Series of Dust Observations at 60640lllizi

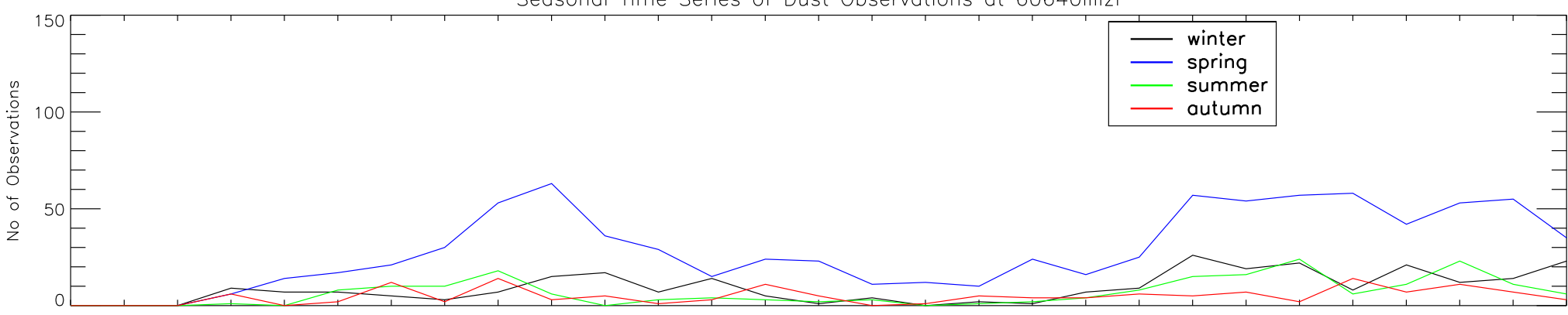

19841985198619871988198919901991199219931994199519961997199819992000200120022003200420052006200720082009201020112012 Time 
Time Series of All Observations at 60656Tindouf

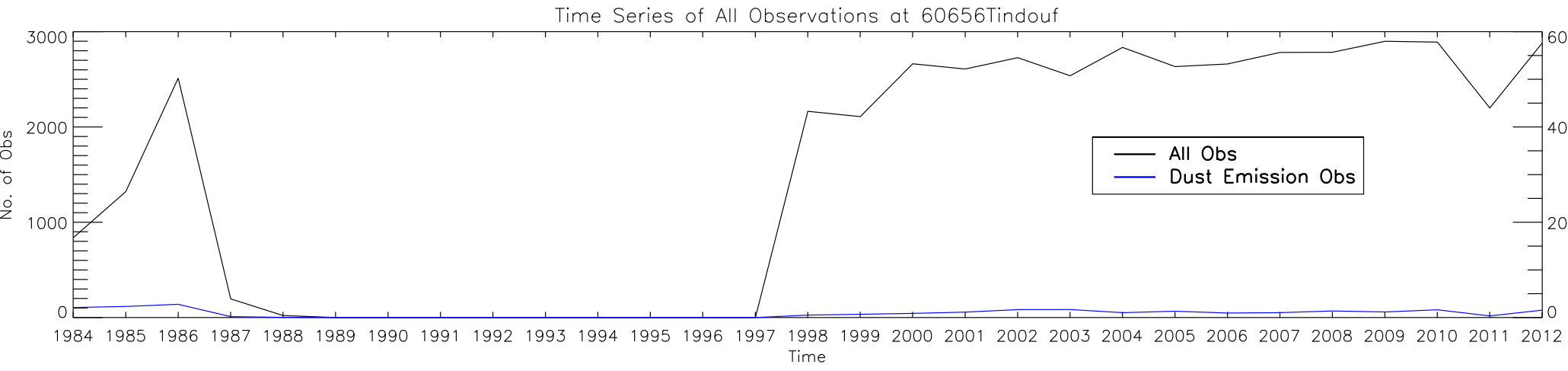

Seasonal Time Series of All Observations at 60656Tindouf

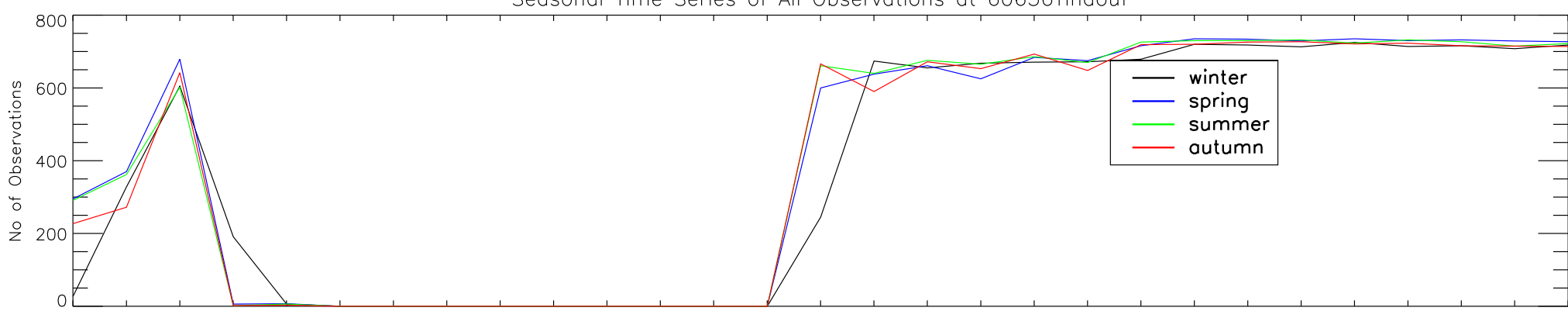

19841985198619871988198919901991199219931994199519961997199819992000200120022003200420052006200720082009201020112012

Seasonal Time Series of Dust Observations at 60656Tindouf

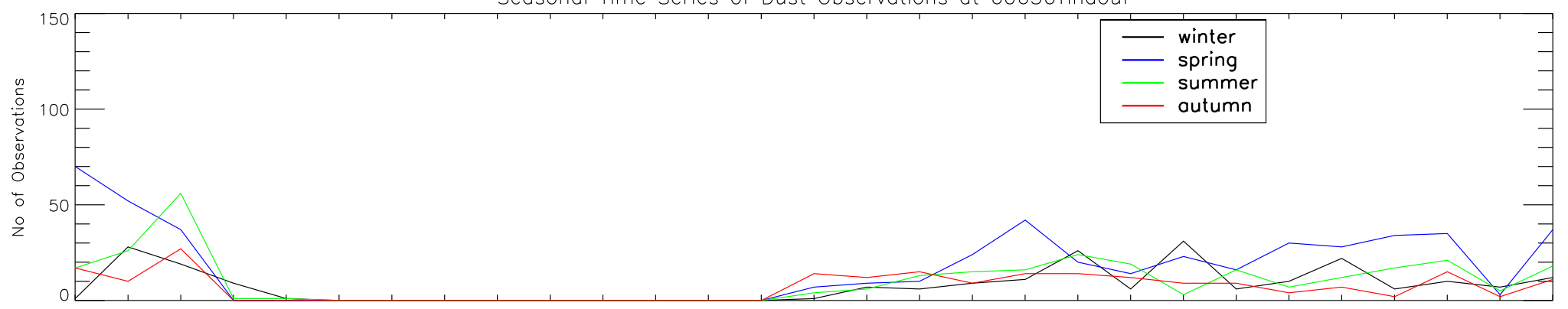

19841985198619871988198919901991199219931994199519961997199819992000200120022003200420052006200720082009201020112012 Time 
Time Series of All Observations at 60686BordjMokhtar
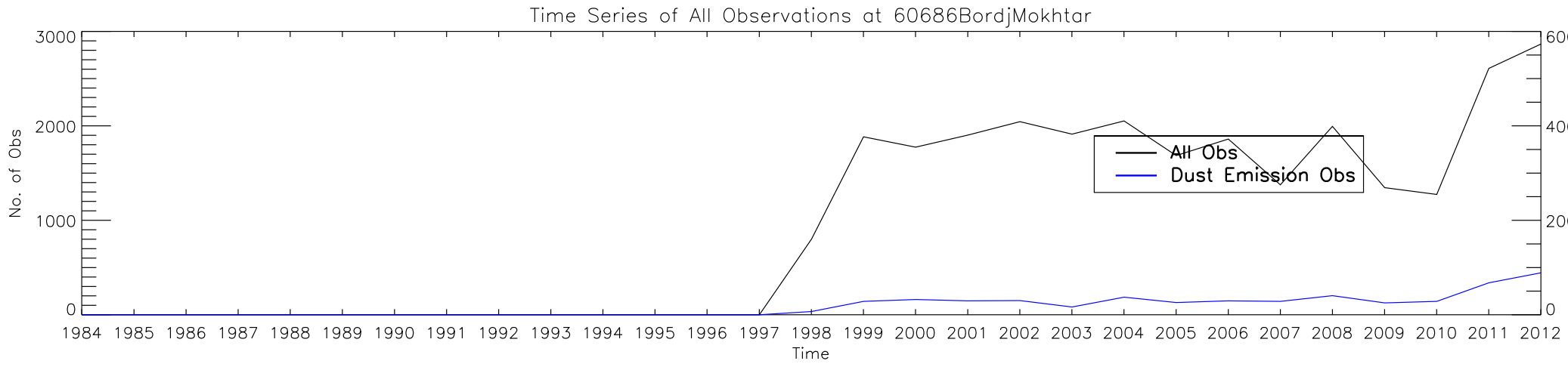

Seasonal Time Series of All Observations at 60686BordjMokhtar

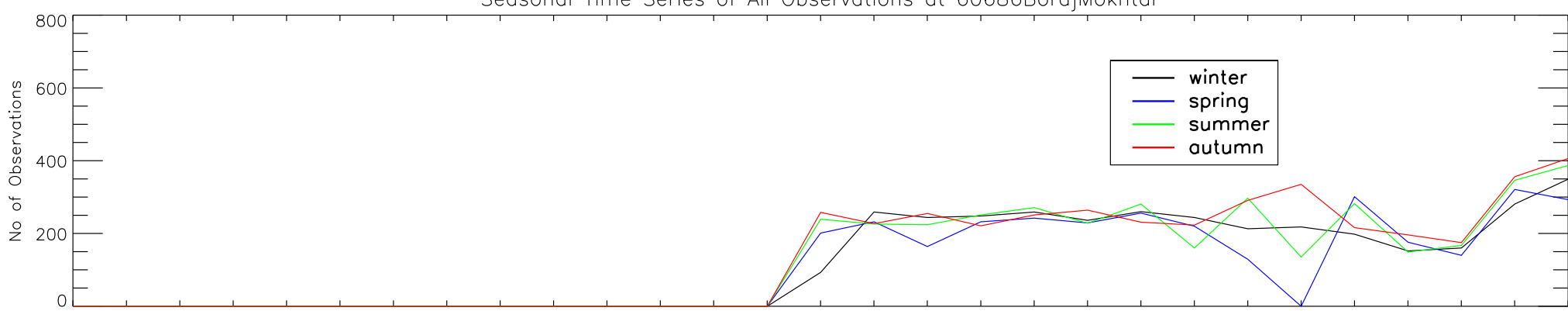

19841985198619871988198919901991199219931994199519961997199819992000200120022003200420052006200720082009201020112012 Time

Seasonal Time Series of Dust Observations at 60686BordjMokhtar

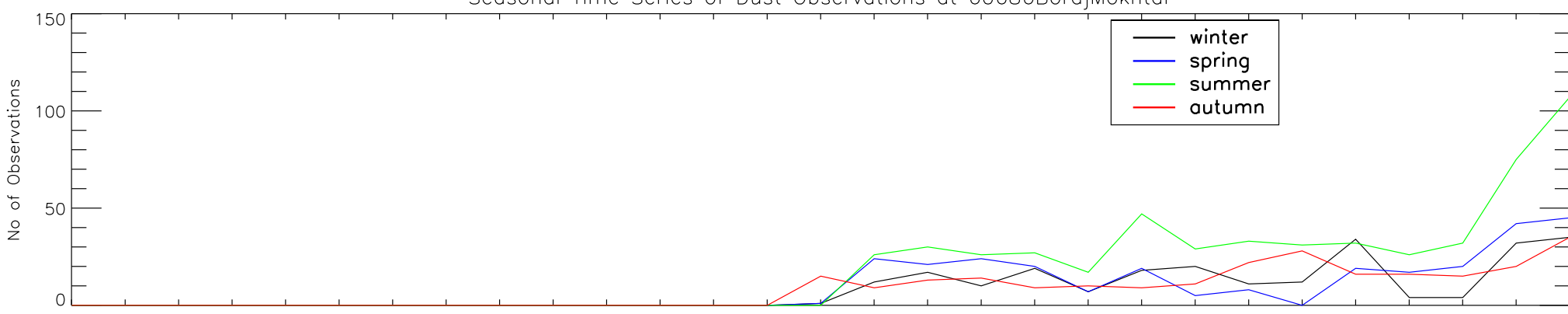

19841985198619871988198919901991199219931994199519961997199819992000200120022003200420052006200720082009201020112012 Time 
 \\ $3000 F_{1}$}

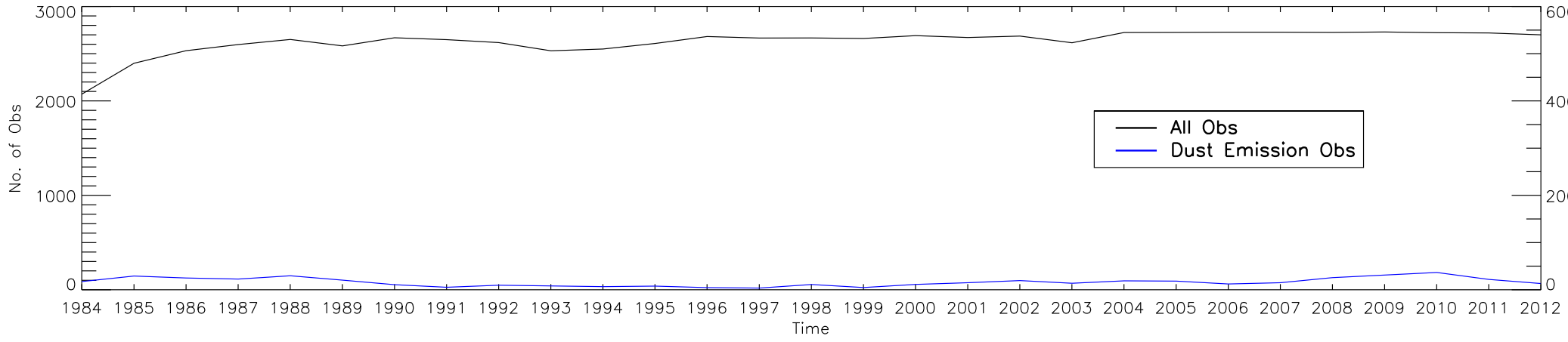

Seasonal Time Series of All Observations at 60775Remada

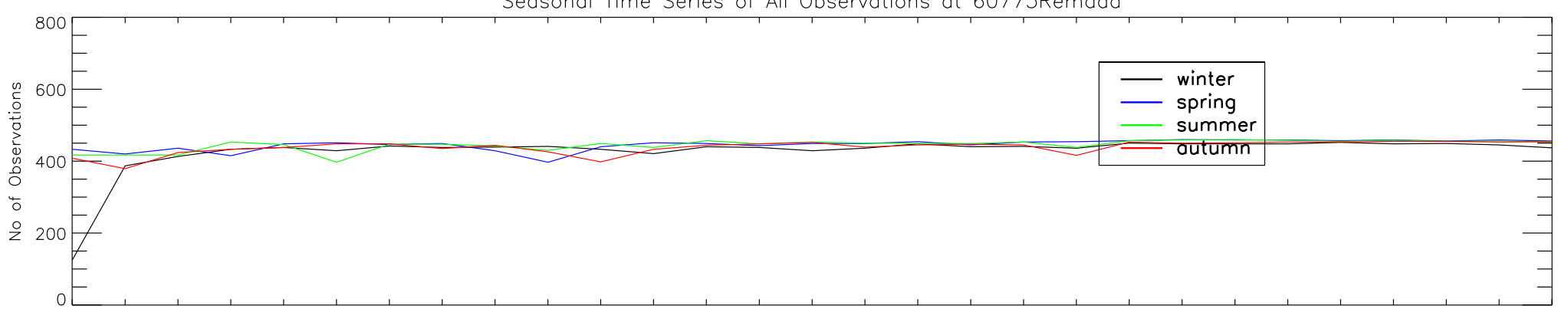

19841985198619871988198919901991199219931994199519961997199819992000200120022003200420052006200720082009201020112012

Seasonal Time Series of Dust Observations at 60775Remada

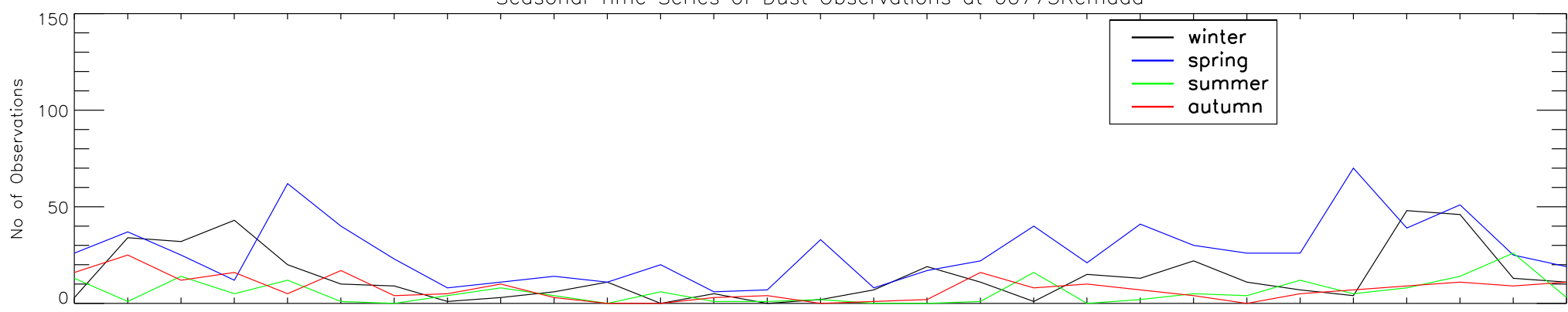

19841985198619871988198919901991199219931994199519961997199819992000200120022003200420052006200720082009201020112012 Time 

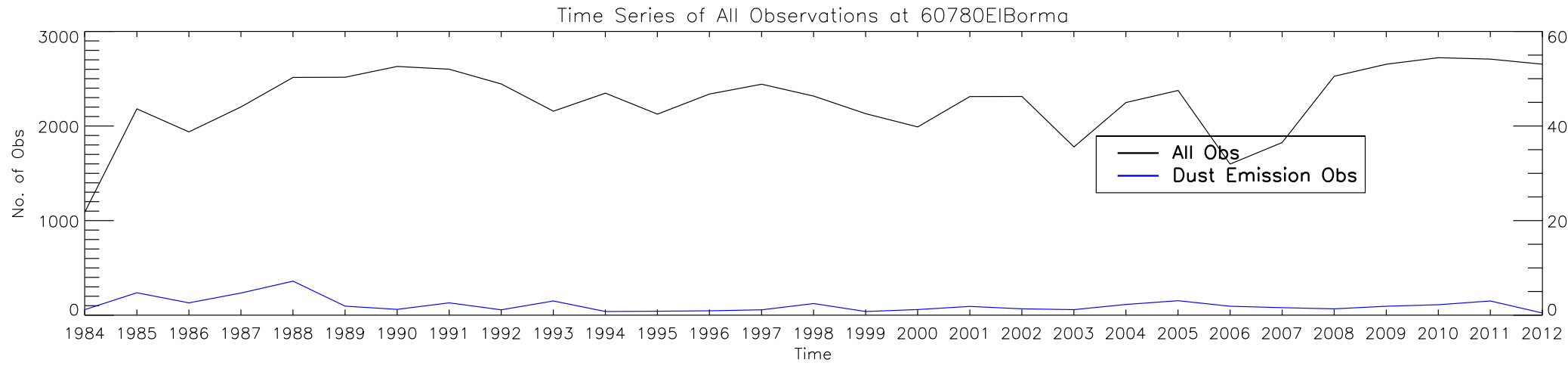

Seasonal Time Series of All Observations at 60780EIBorma

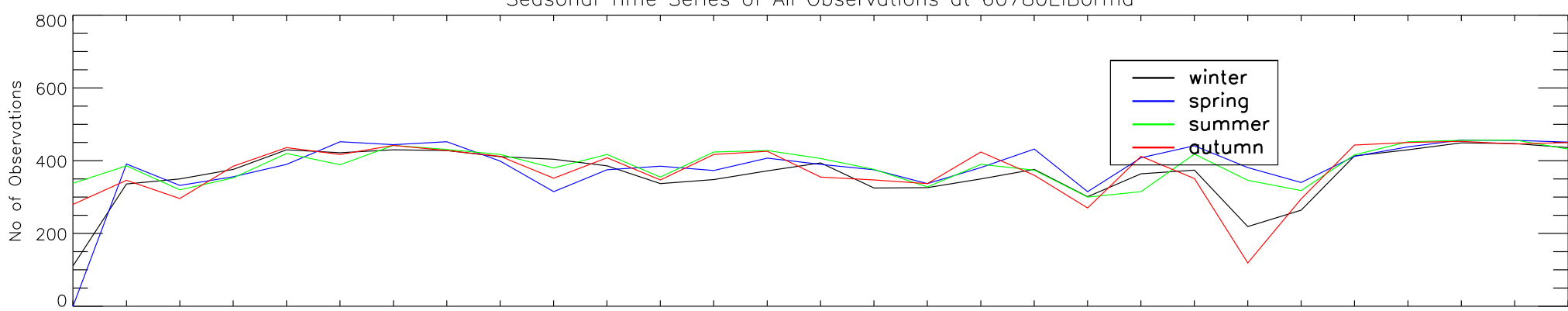

19841985198619871988198919901991199219931994199519961997199819992000200120022003200420052006200720082009201020112012

Seasonal Time Series of Dust Observations at 60780EIBorma

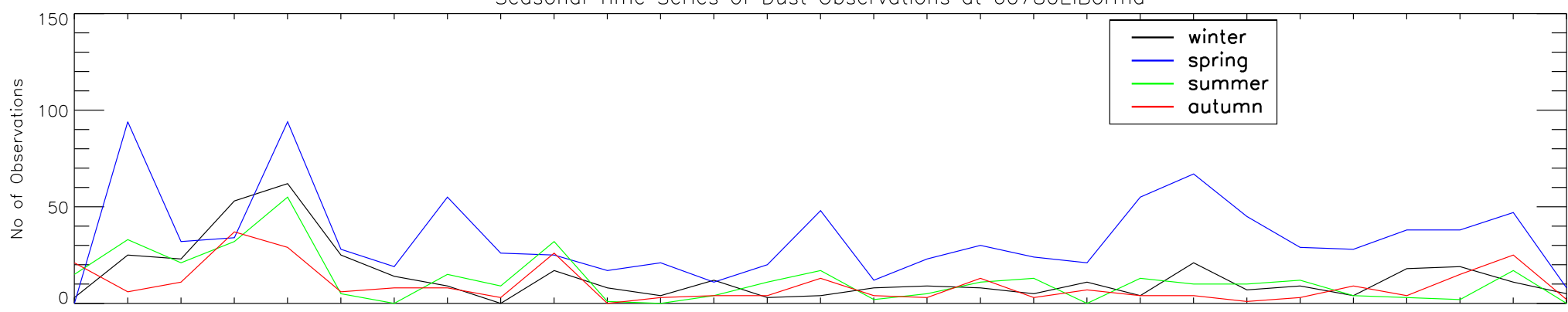

19841985198619871988198919901991199219931994199519961997199819992000200120022003200420052006200720082009201020112012 Time 


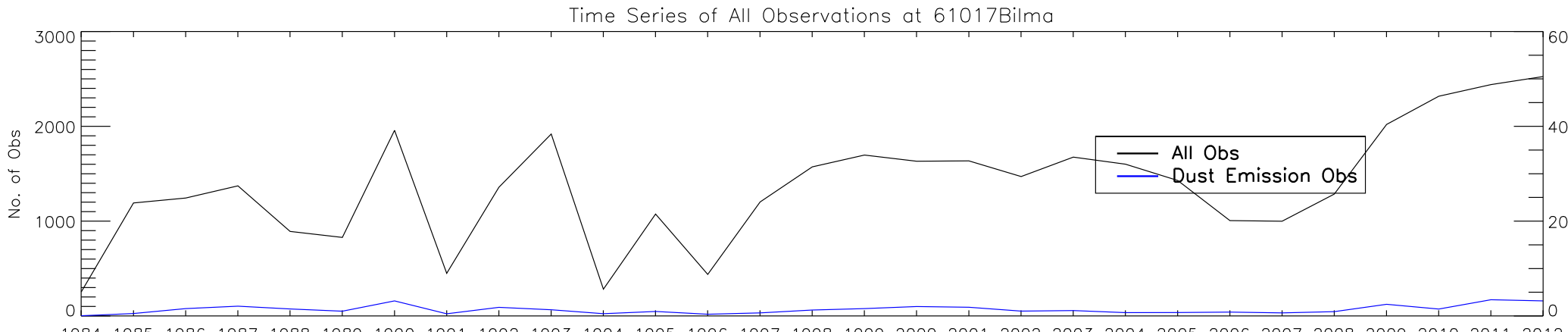

19841985198619871988198919901991199219931994199519961997199819992000200120022003200420052006200720082009201020112012 Time

Seasonal Time Series of All Observations at 61017Bilma

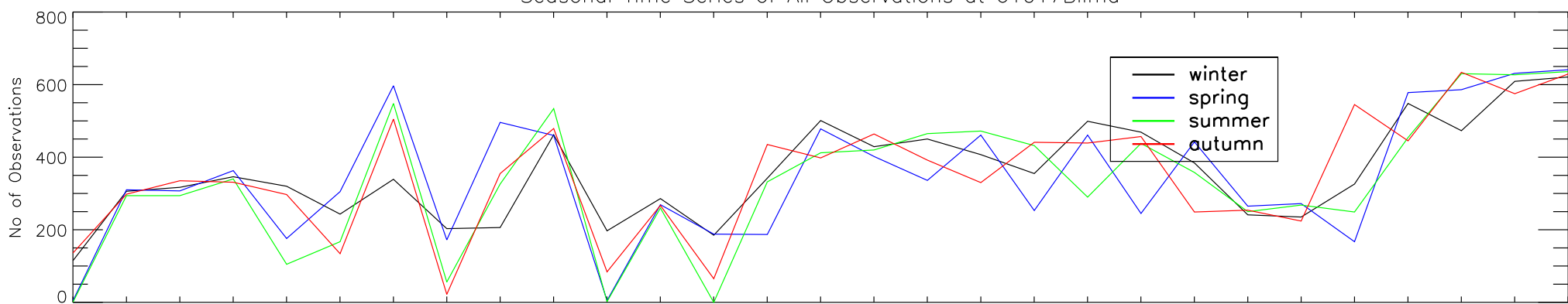

19841985198619871988198919901991199219931994199519961997199819992000200120022003200420052006200720082009201020112012

Seasonal Time Series of Dust Observations at 61017Bilma

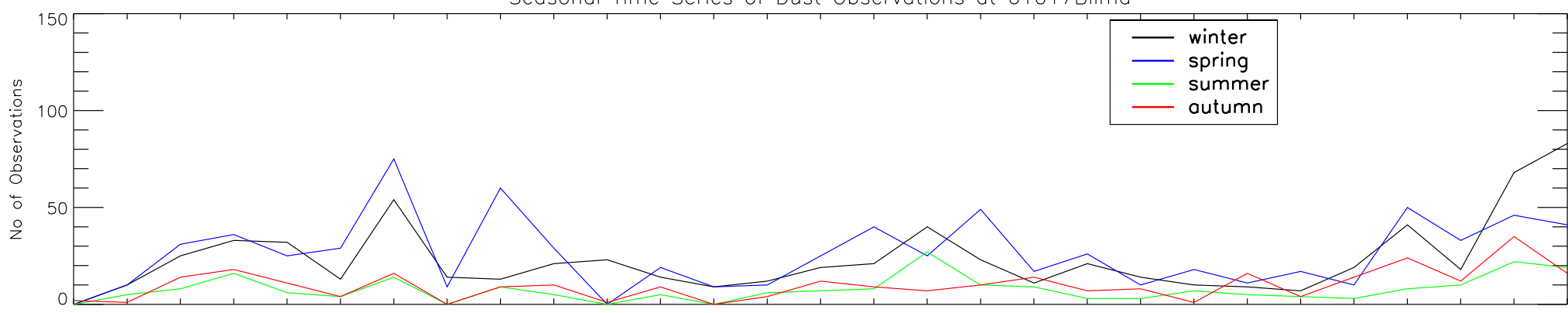

19841985198619871988198919901991199219931994199519961997199819992000200120022003200420052006200720082009201020112012 Time 


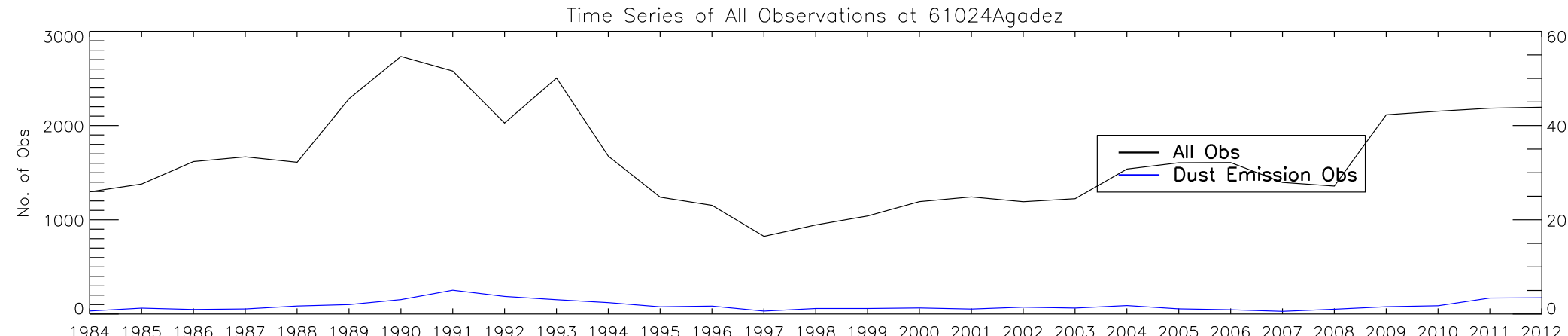

19841985198619871988198919901991199219931994199519961997199819992000200120022003200420052006200720082009201020112012 Time

Seasonal Time Series of All Observations at 61024Agadez

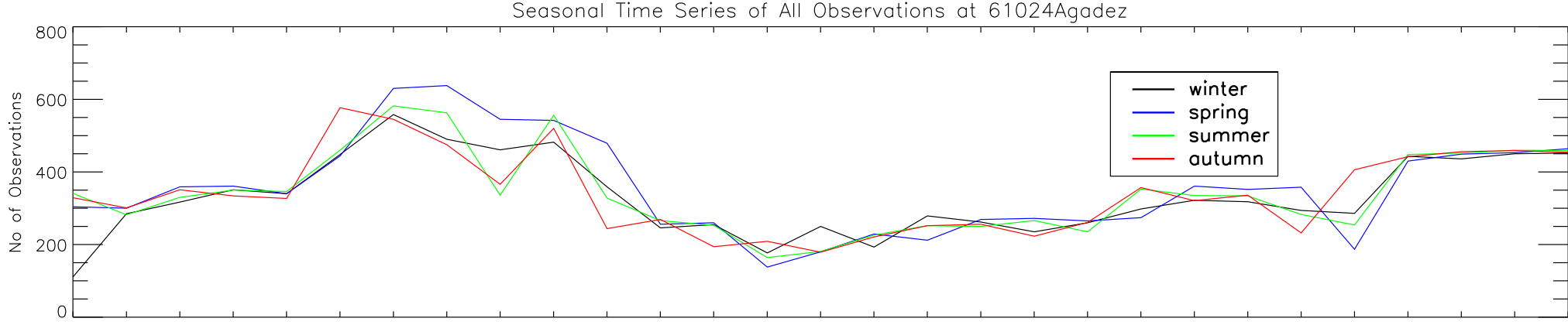

19841985198619871988198919901991199219931994199519961997199819992000200120022003200420052006200720082009201020112012

Seasonal Time Series of Dust Observations at 61024Agadez

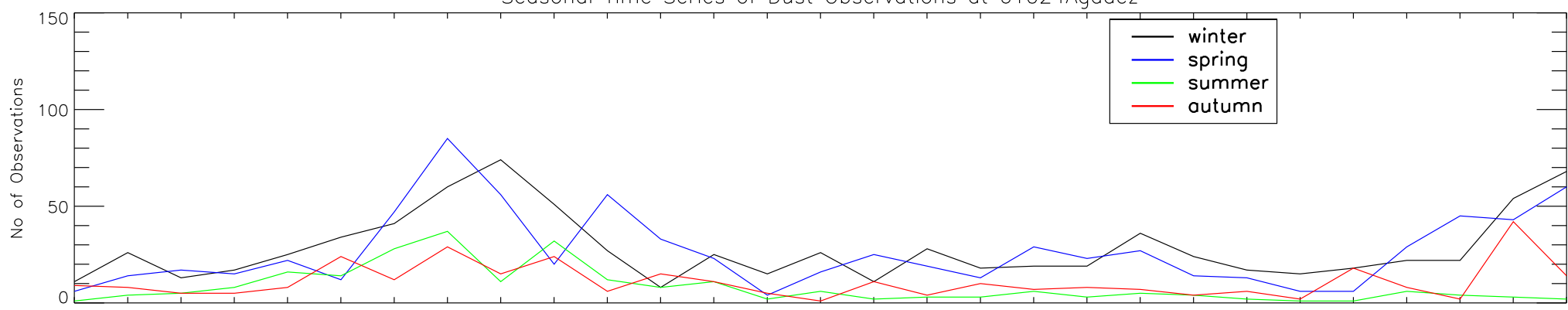

19841985198619871988198919901991199219931994199519961997199819992000200120022003200420052006200720082009201020112012 Time 


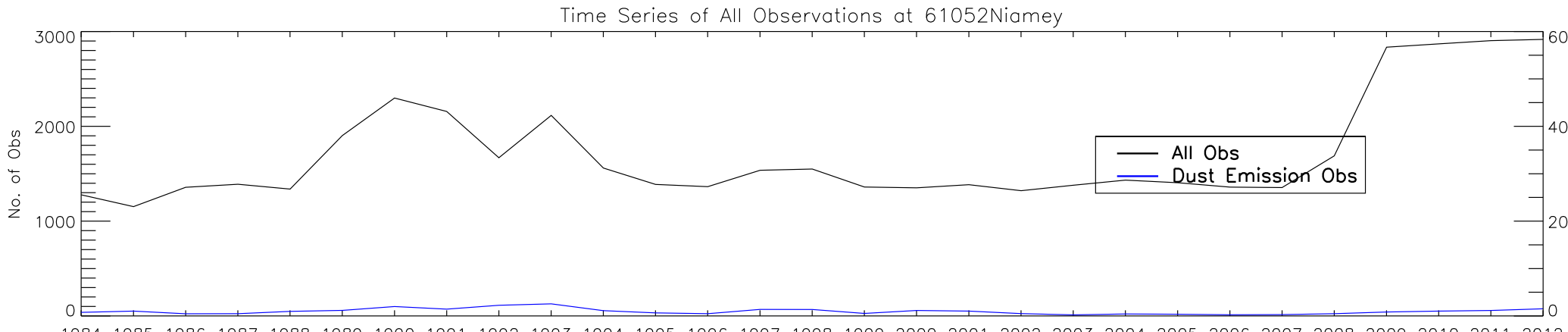

19841985198619871988198919901991199219931994199519961997199819992000200120022003200420052006200720082009201020112012 Time

Seasonal Time Series of All Observations at 61052Niamey

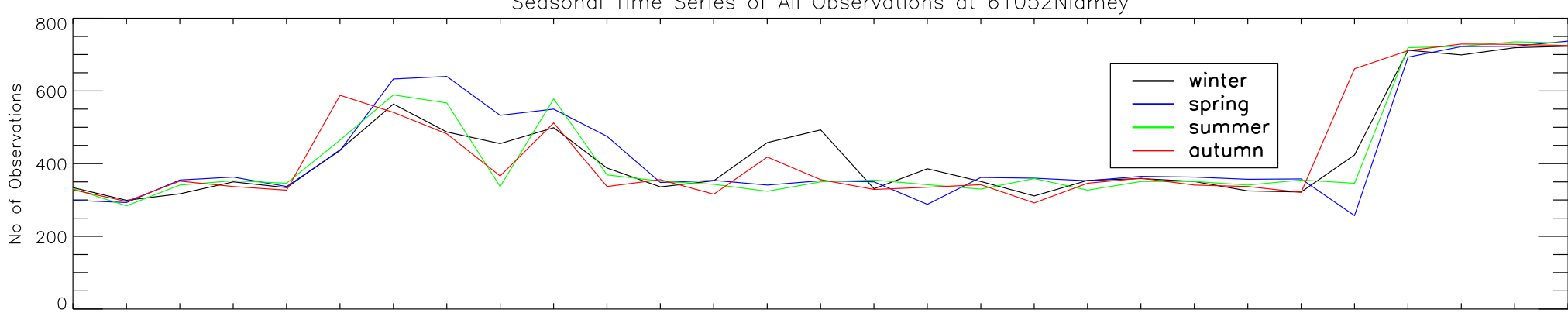

19841985198619871988198919901991199219931994199519961997199819992000200120022003200420052006200720082009201020112012

Seasonal Time Series of Dust Observations at 61052Niamey

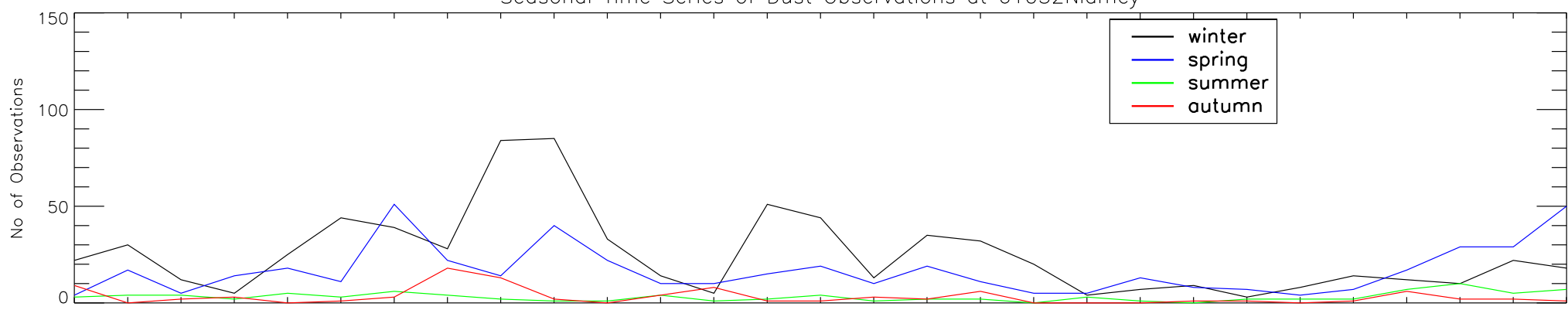

19841985198619871988198919901991199219931994199519961997199819992000200120022003200420052006200720082009201020112012 Time 
Time Series of All Observations at 61223Tombouctou

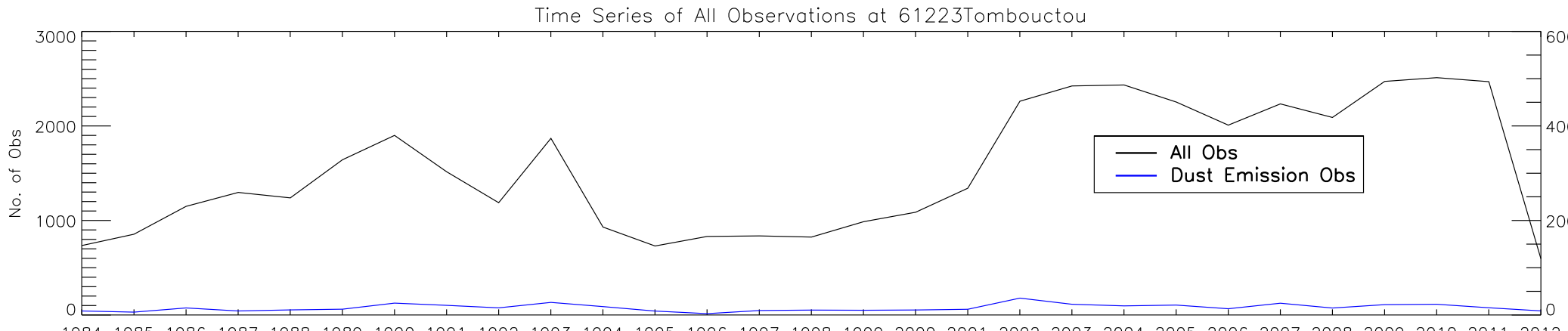

19841985198619871988198919901991199219931994199519961997199819992000200120022003200420052006200720082009201020112012

Seasonal Time Series of All Observations at 61223Tombouctou

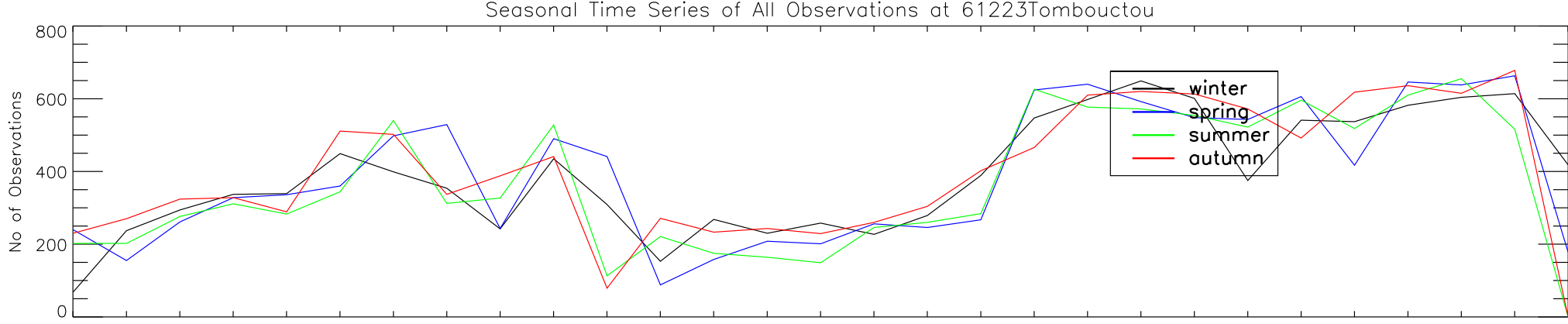

19841985198619871988198919901991199219931994199519961997199819992000200120022003200420052006200720082009201020112012

Seasonal Time Series of Dust Observations at 61223Tombouctou

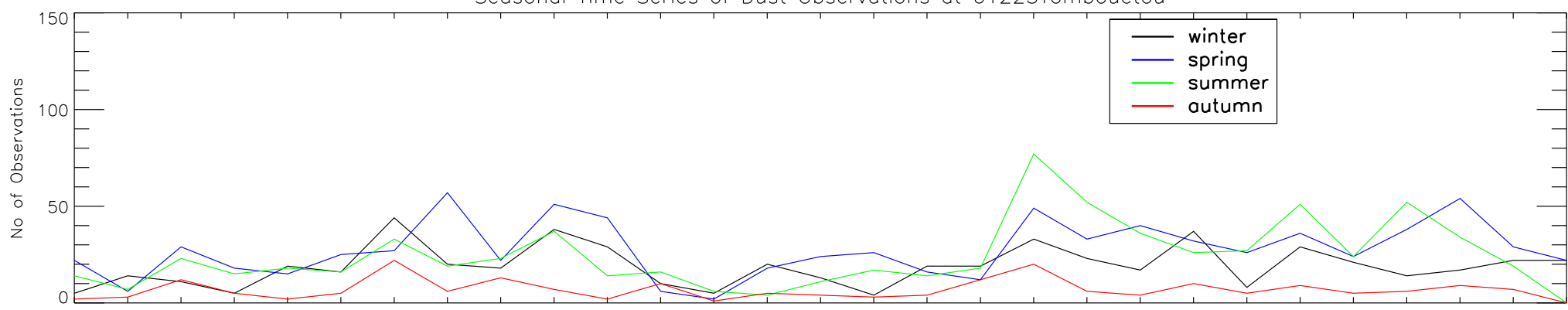

19841985198619871988198919901991199219931994199519961997199819992000200120022003200420052006200720082009201020112012 Time 
Time Series of All Observations at 61230NioroDuSahel

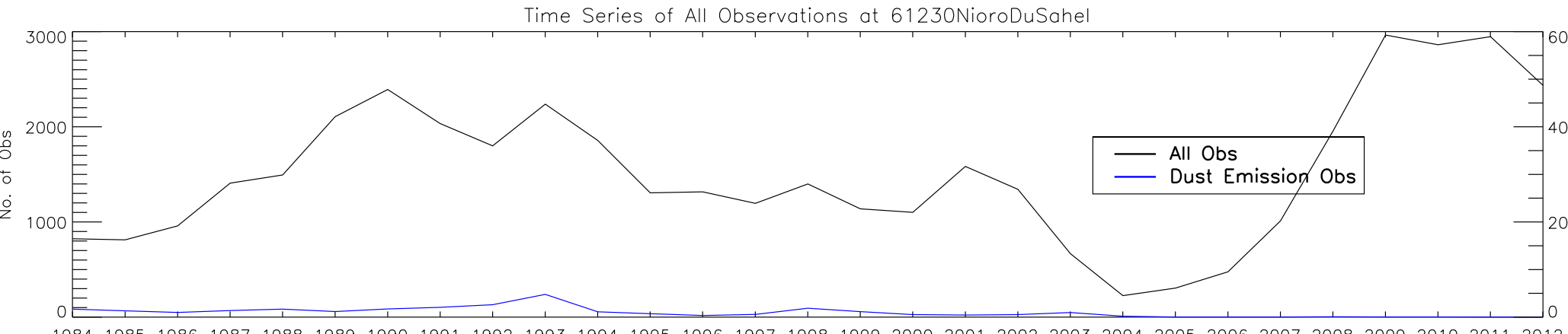

19841985198619871988198919901991199219931994199519961997199819992000200120022003200420052006200720082009201020112012 Time

Seasonal Time Series of All Observations at 61230NioroDuSahel

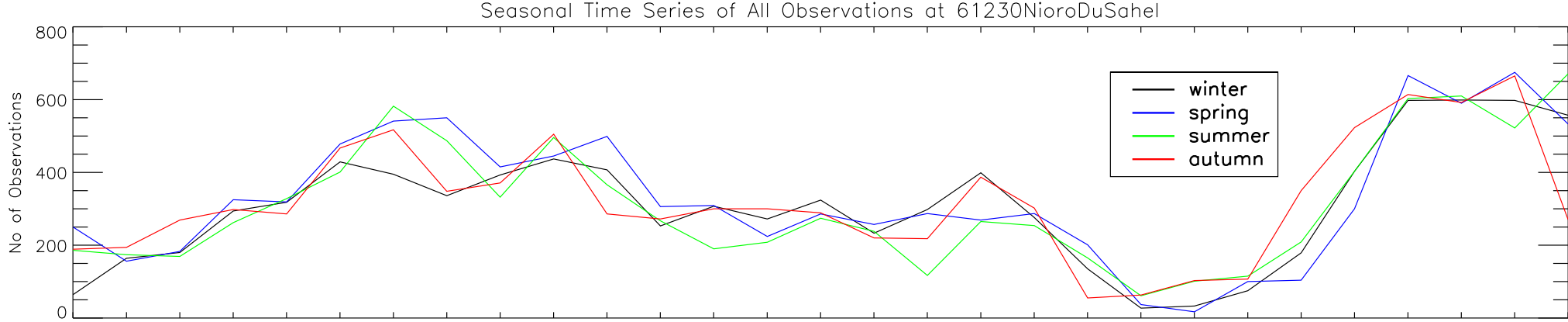

19841985198619871988198919901991199219931994199519961997199819992000200120022003200420052006200720082009201020112012

Seasonal Time Series of Dust Observations at 61230NioroDuSahel

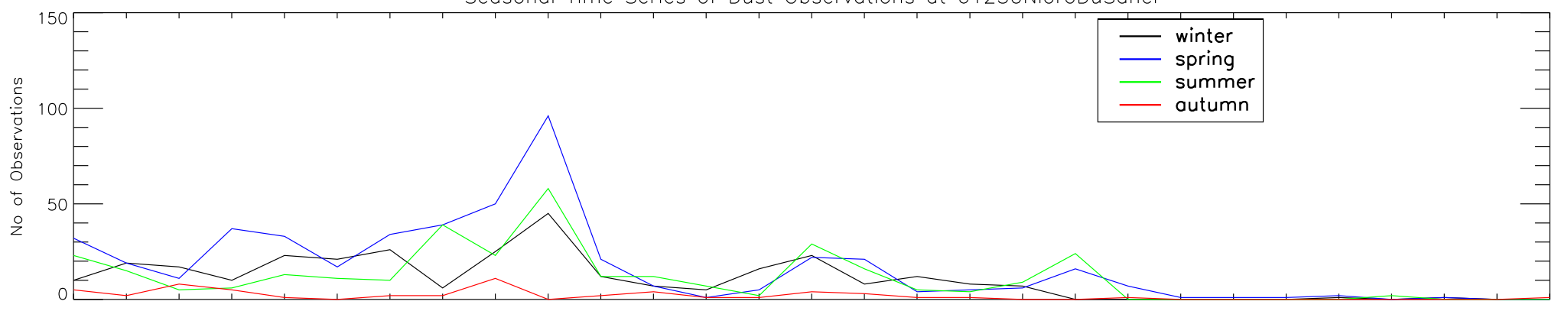

19841985198619871988198919901991199219931994199519961997199819992000200120022003200420052006200720082009201020112012 Time 
Time Series of All Observations at 61404Zouerate

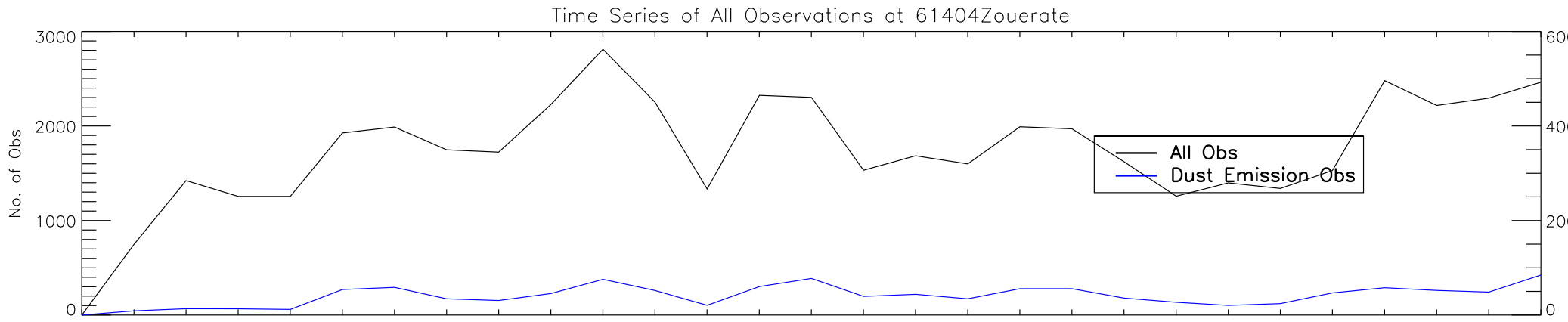

19841985198619871988198919901991199219931994199519961997199819992000200120022003200420052006200720082009201020112012 Time

Seasonal Time Series of All Observations at 61404 Zouerate

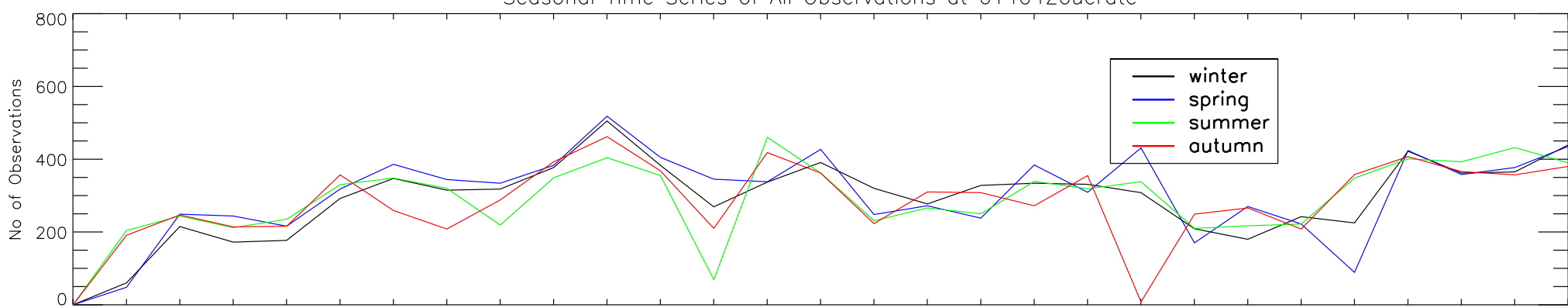

19841985198619871988198919901991199219931994199519961997199819992000200120022003200420052006200720082009201020112012

Seasonal Time Series of Dust Observations at 61404 Zouerate

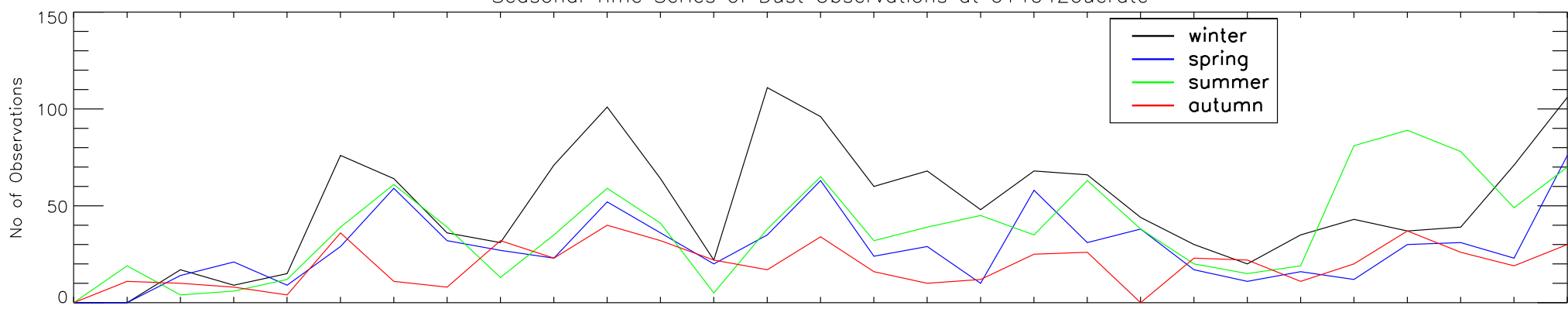

19841985198619871988198919901991199219931994199519961997199819992000200120022003200420052006200720082009201020112012 Time 


\section{0}

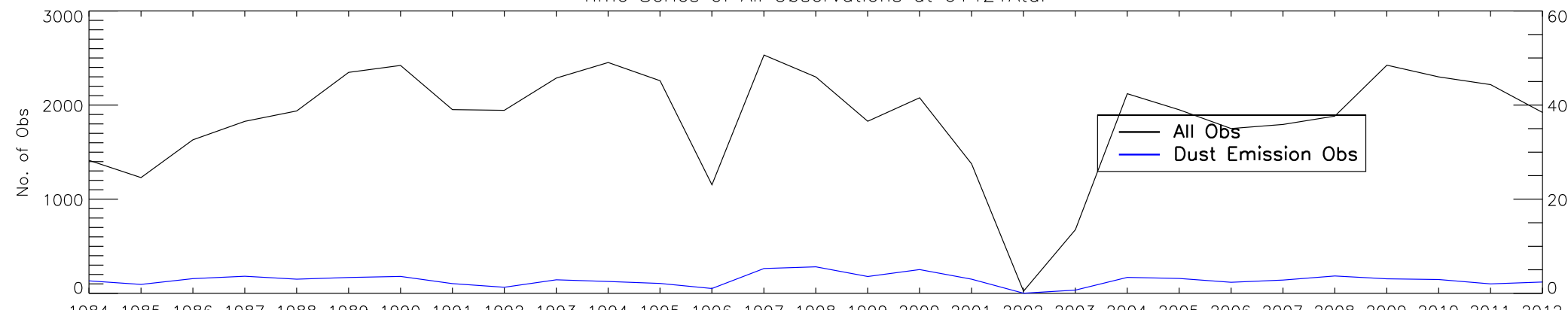

19841985198619871988198919901991199219931994199519961997199819992000200120022003200420052006200720082009201020112012 Time

Seasonal Time Series of All Observations at 61421Atar

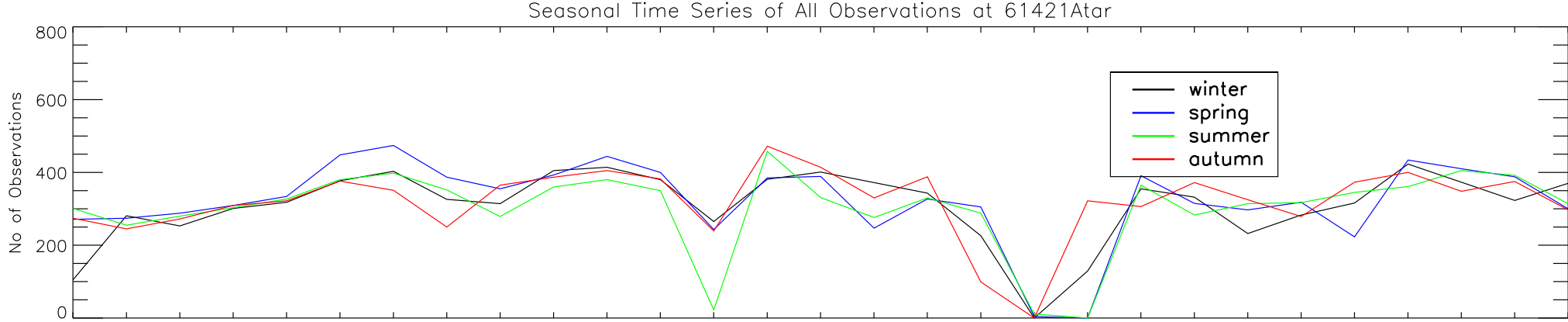

19841985198619871988198919901991199219931994199519961997199819992000200120022003200420052006200720082009201020112012

Seasonal Time Series of Dust Observations at 61421Atar

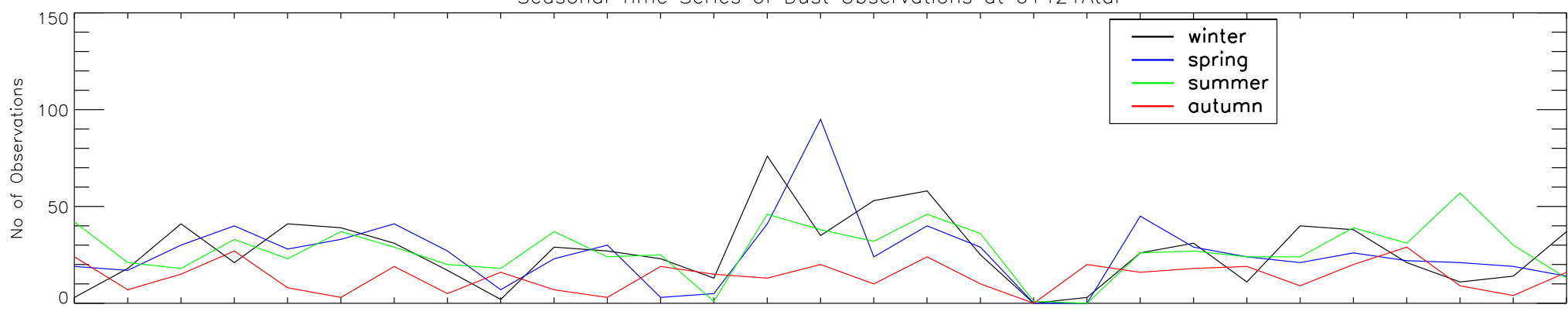

19841985198619871988198919901991199219931994199519961997199819992000200120022003200420052006200720082009201020112012 Time 
Time Series of All Observations at 61437Akjoujt

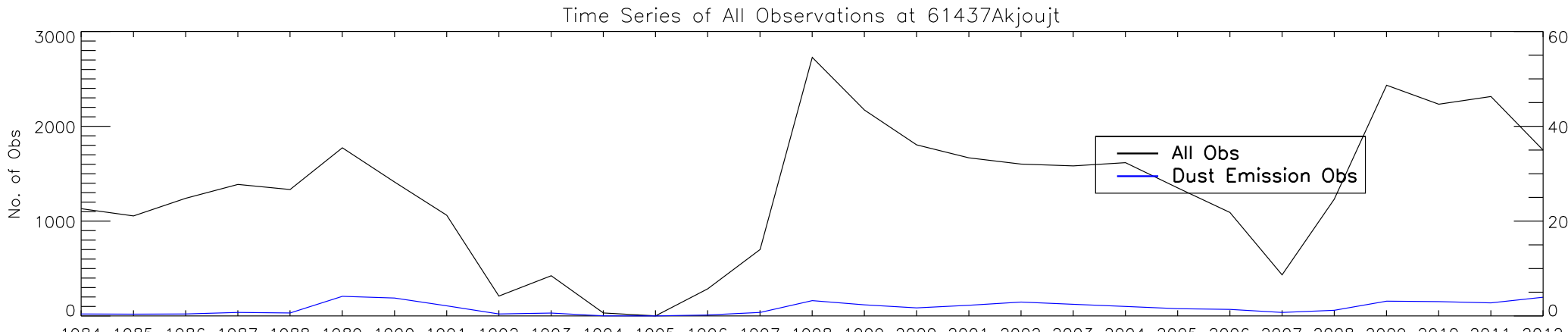

19841985198619871988198919901991199219931994199519961997199819992000200120022003200420052006200720082009201020112012 Time

Seasonal Time Series of All Observations at 61437Akjoujt

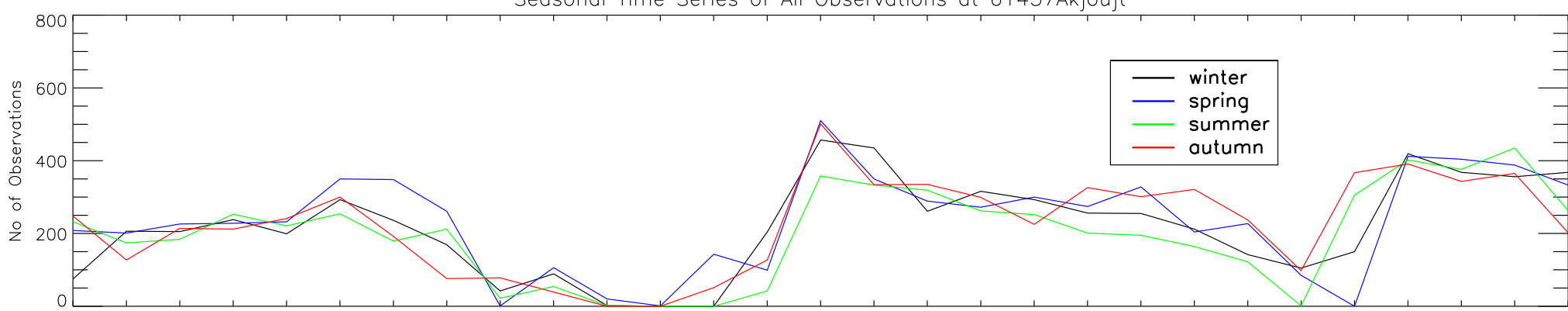

19841985198619871988198919901991199219931994199519961997199819992000200120022003200420052006200720082009201020112012

Seasonal Time Series of Dust Observations at 61437Akjoujt

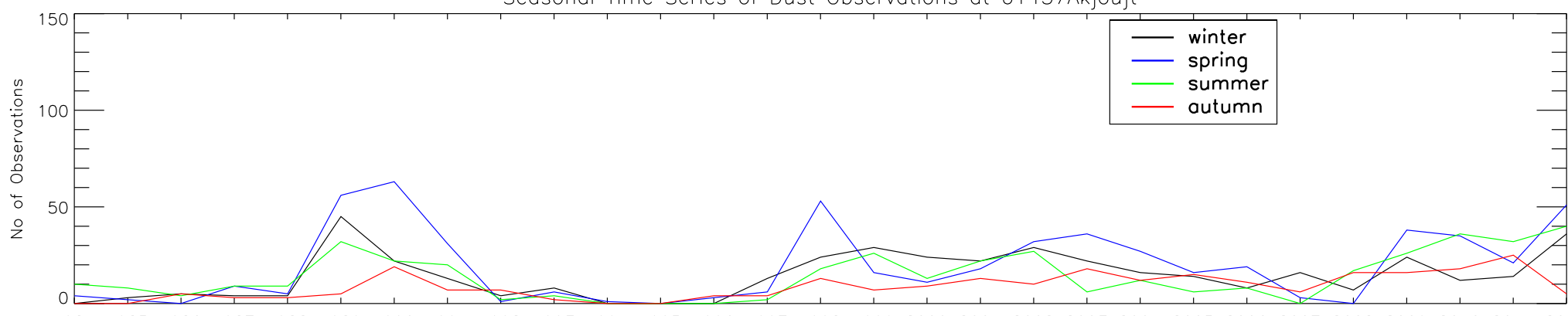

19841985198619871988198919901991199219931994199519961997199819992000200120022003200420052006200720082009201020112012 


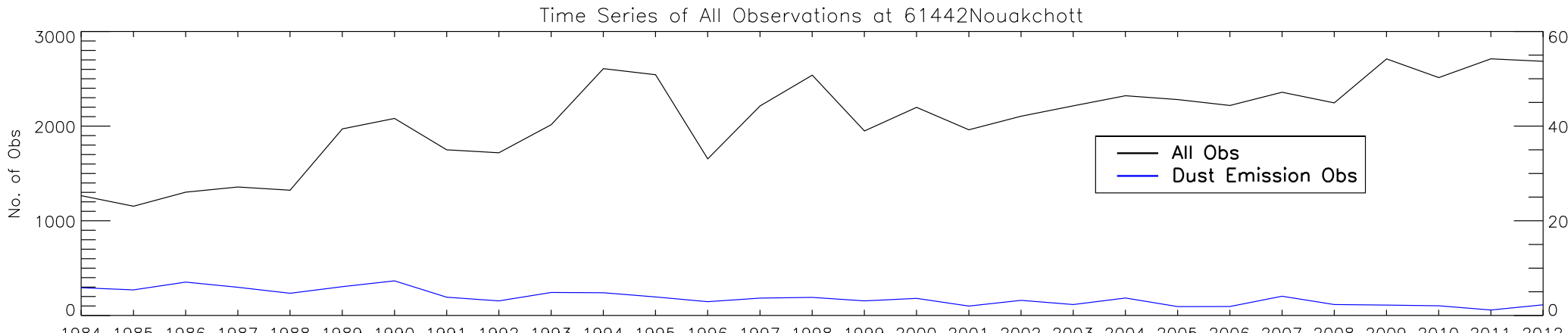

19841985198619871988198919901991199219931994199519961997199819992000200120022003200420052006200720082009201020112012 Time

Seasonal Time Series of All Observations at 61442Nouakchott

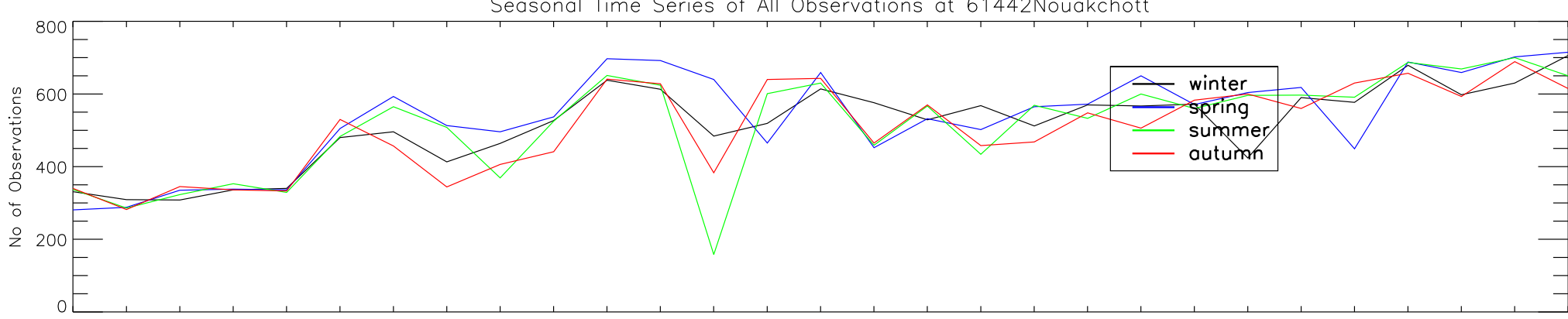

19841985198619871988198919901991199219931994199519961997199819992000200120022003200420052006200720082009201020112012

Seasonal Time Series of Dust Observations at 61442Nouakchott

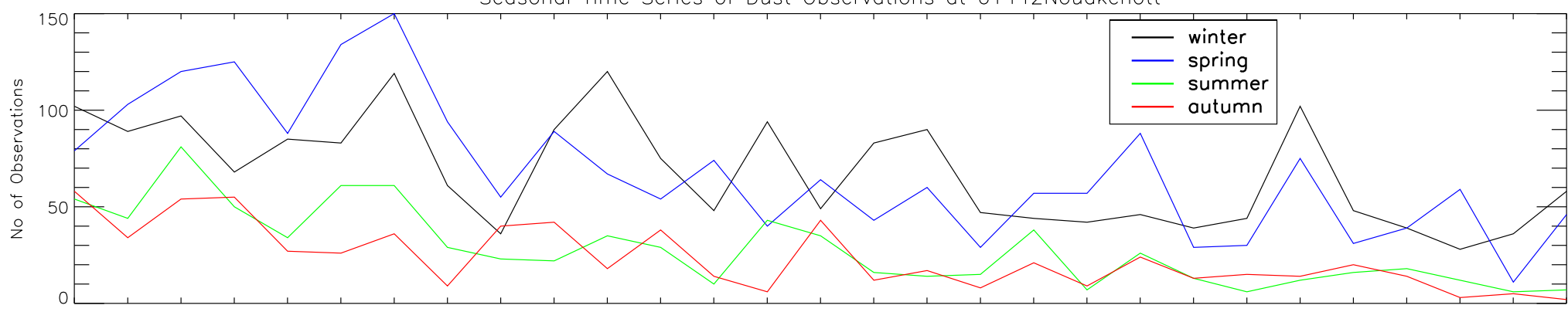

19841985198619871988198919901991199219931994199519961997199819992000200120022003200420052006200720082009201020112012 Time 
Time Series of All Observations at 61450Tidjika

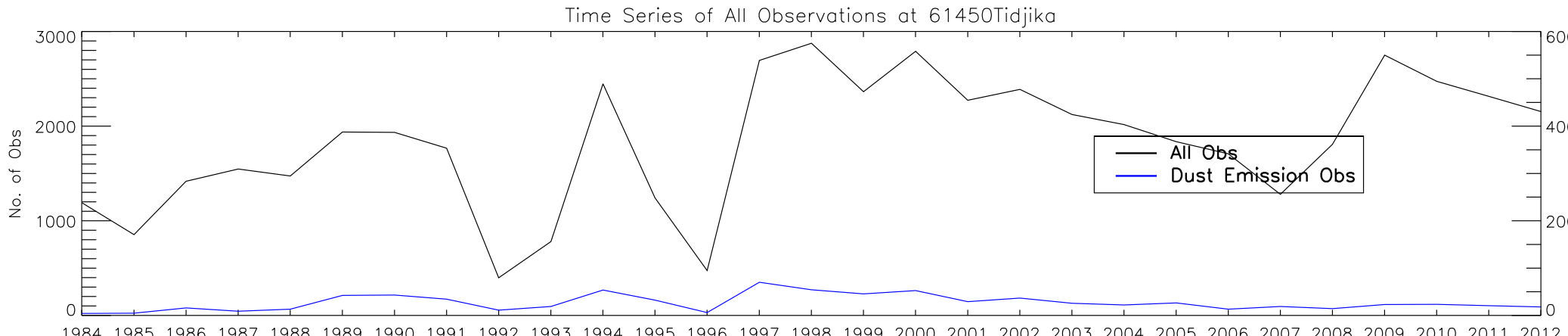

19841985198619871988198919901991199219931994199519961997199819992000200120022003200420052006200720082009201020112012 Time

Seasonal Time Series of All Observations at 61450Tidjika

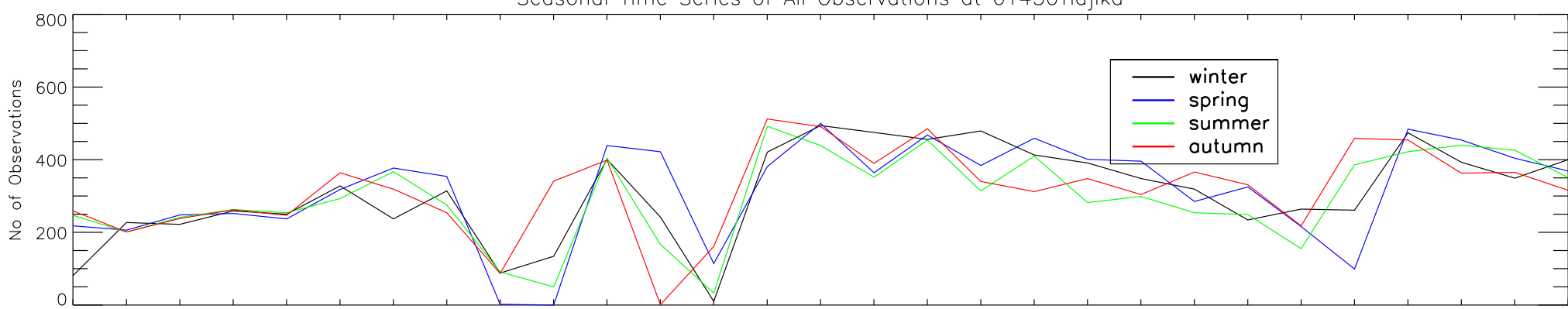

19841985198619871988198919901991199219931994199519961997199819992000200120022003200420052006200720082009201020112012

Seasonal Time Series of Dust Observations at 61450Tidjika

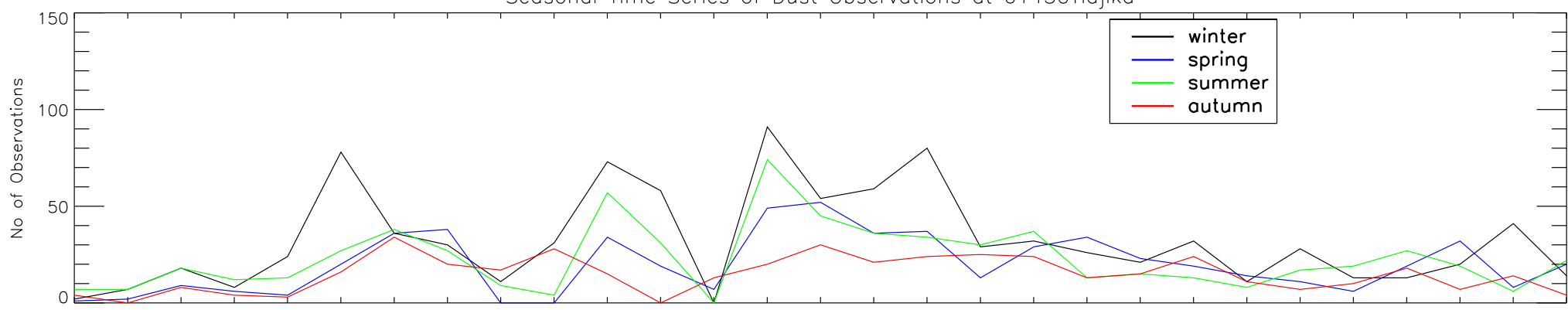

19841985198619871988198919901991199219931994199519961997199819992000200120022003200420052006200720082009201020112012 Time 
 \\ Time Series of All Observations at 61489Rosso}

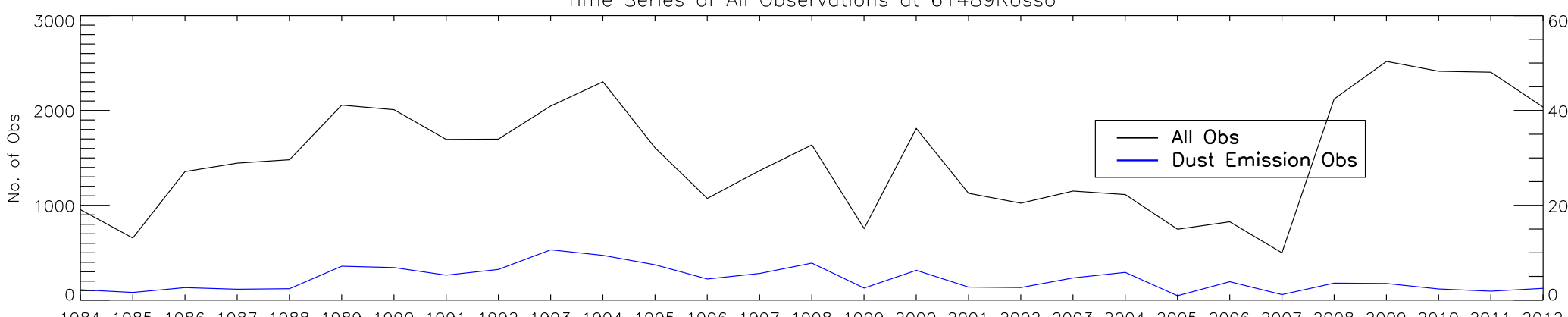

19841985198619871988198919901991199219931994199519961997199819992000200120022003200420052006200720082009201020112012 Time

Seasonal Time Series of All Observations at 61489Rosso

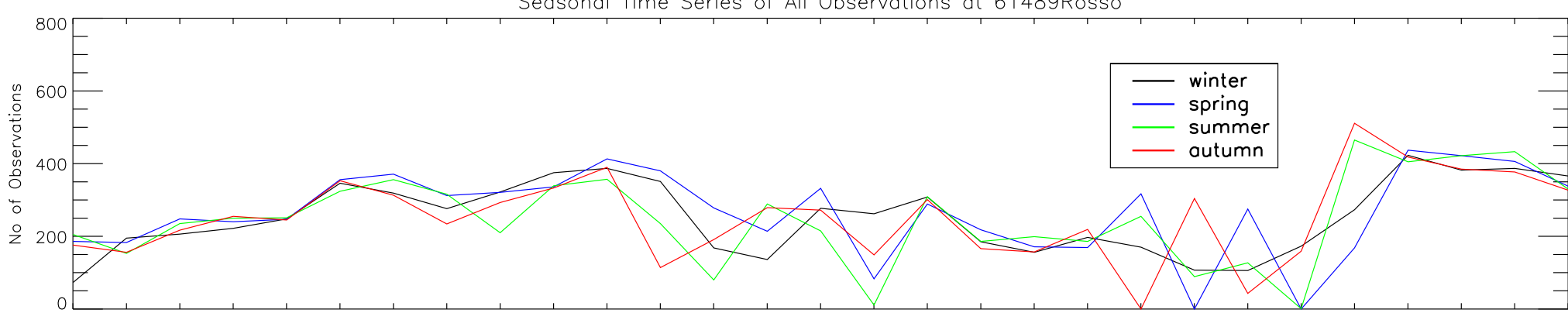

19841985198619871988198919901991199219931994199519961997199819992000200120022003200420052006200720082009201020112012

Seasonal Time Series of Dust Observations at 61489Rosso

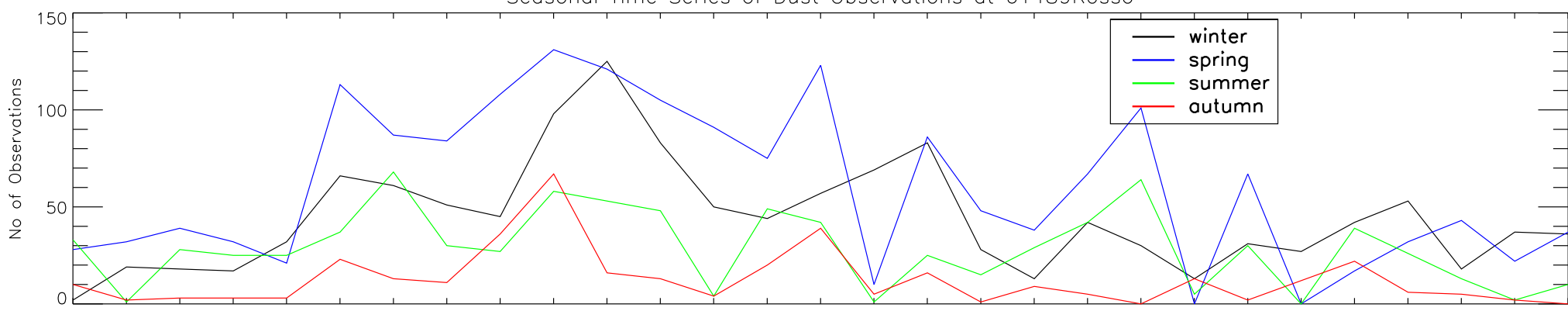

19841985198619871988198919901991199219931994199519961997199819992000200120022003200420052006200720082009201020112012 Time 


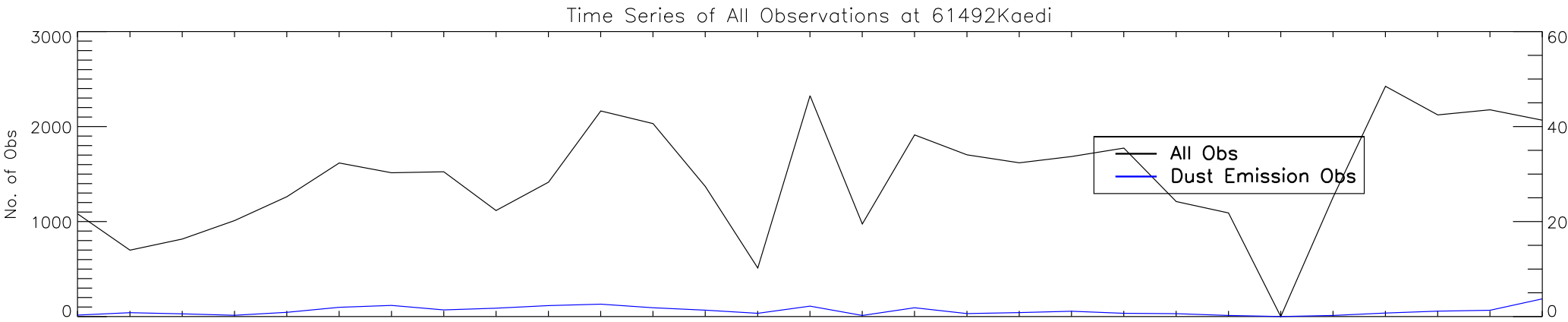

19841985198619871988198919901991199219931994199519961997199819992000200120022003200420052006200720082009201020112012 Time

Seasonal Time Series of All Observations at 61492Kaedi

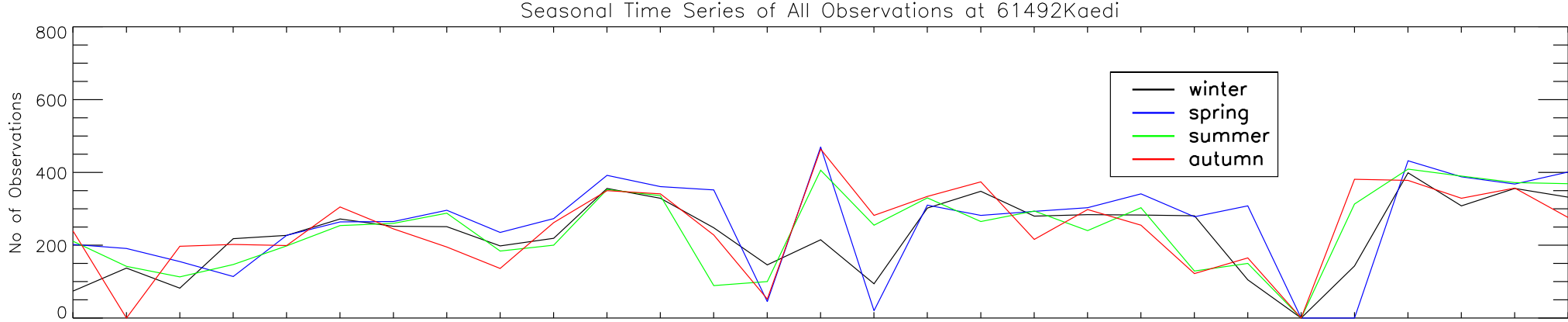

19841985198619871988198919901991199219931994199519961997199819992000200120022003200420052006200720082009201020112012

Seasonal Time Series of Dust Observations at 61492Kaedi

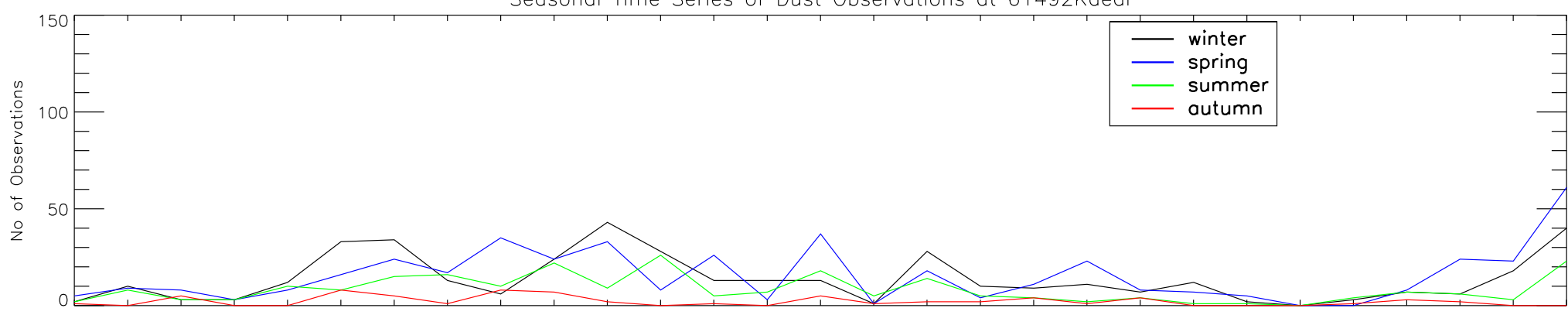

19841985198619871988198919901991199219931994199519961997199819992000200120022003200420052006200720082009201020112012 Time 


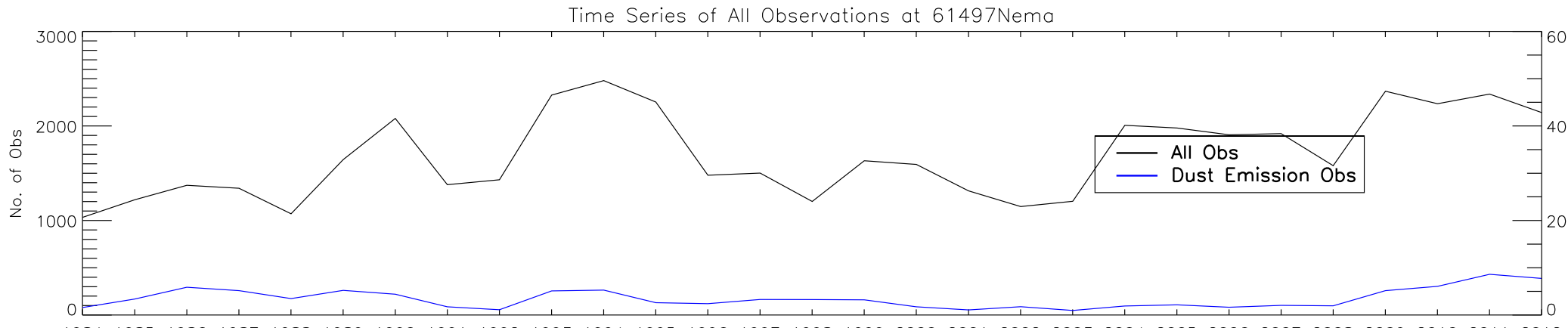

19841985198619871988198919901991199219931994199519961997199819992000200120022003200420052006200720082009201020112012 Time

Seasonal Time Series of All Observations at 61497Nema

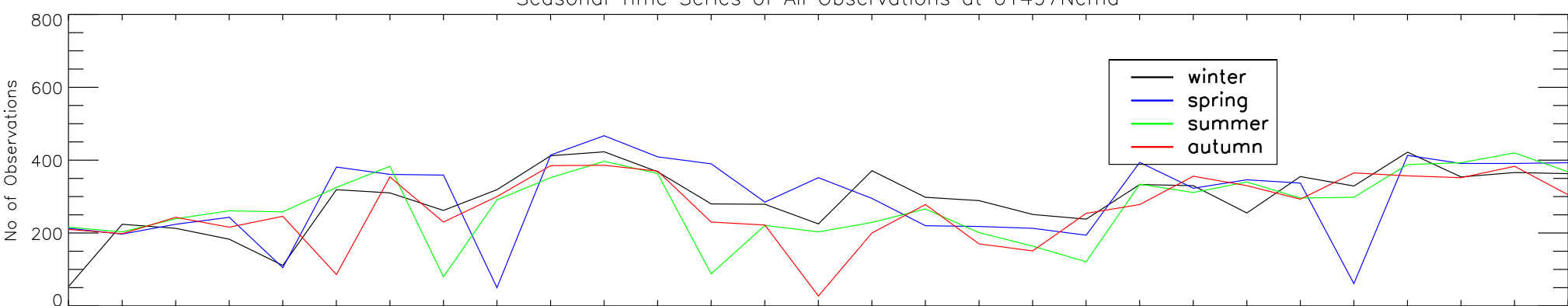

19841985198619871988198919901991199219931994199519961997199819992000200120022003200420052006200720082009201020112012

Seasonal Time Series of Dust Observations at 61497Nema

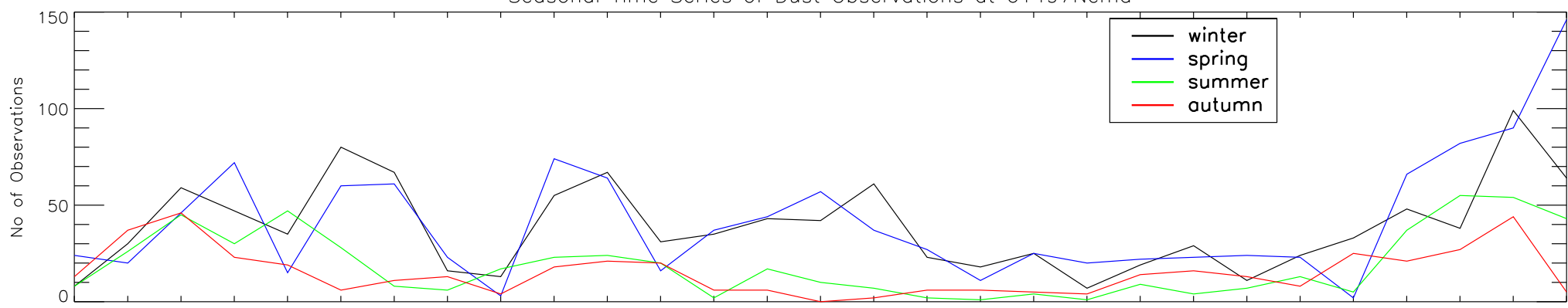

19841985198619871988198919901991199219931994199519961997199819992000200120022003200420052006200720082009201020112012 Time 
Time Series of All Observations at 61499AiounEIAtrouss

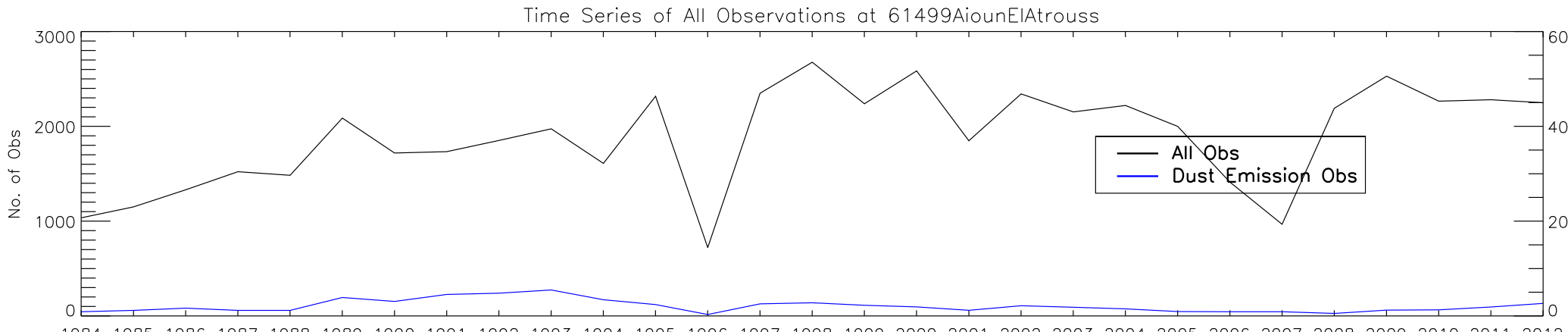

19841985198619871988198919901991199219931994199519961997199819992000200120022003200420052006200720082009201020112012

Seasonal Time Series of All Observations at 61499AiounElAtrouss

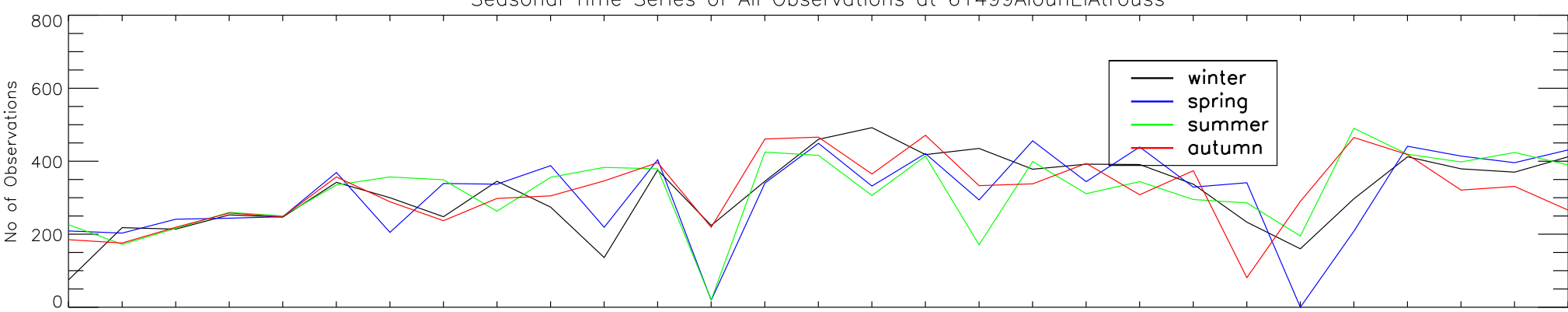

19841985198619871988198919901991199219931994199519961997199819992000200120022003200420052006200720082009201020112012

Seasonal Time Series of Dust Observations at 61499AiounEIAtrouss

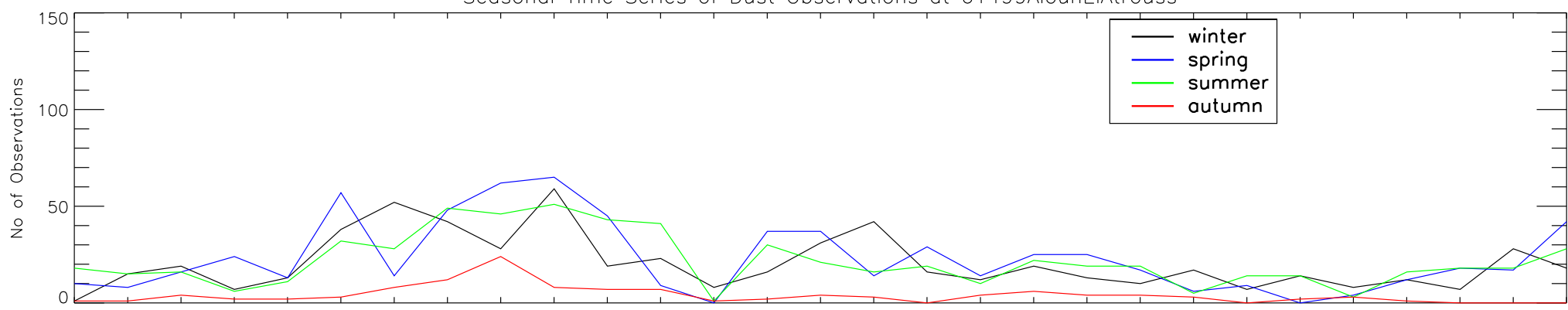

19841985198619871988198919901991199219931994199519961997199819992000200120022003200420052006200720082009201020112012 Time 


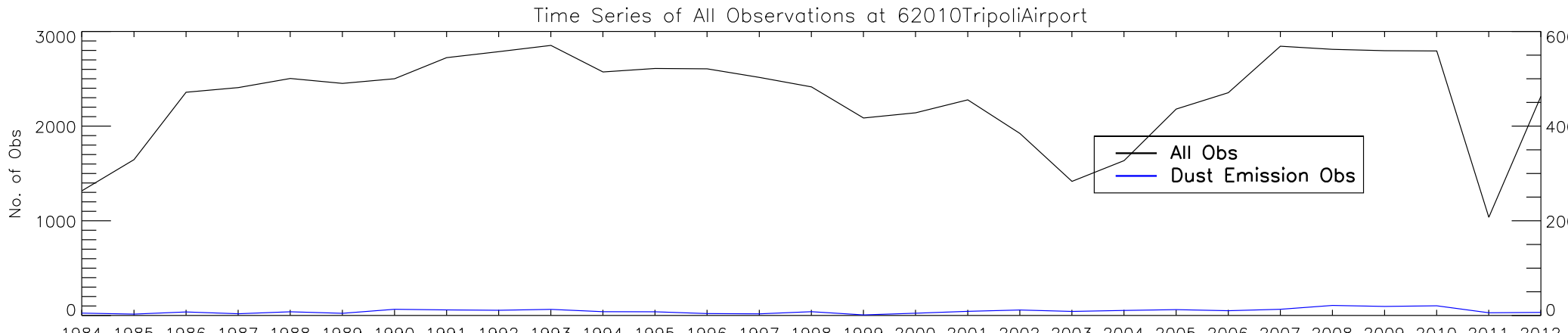

19841985198619871988198919901991199219931994199519961997199819992000200120022003200420052006200720082009201020112012 Time

Seasonal Time Series of All Observations at 62010TripoliAirport

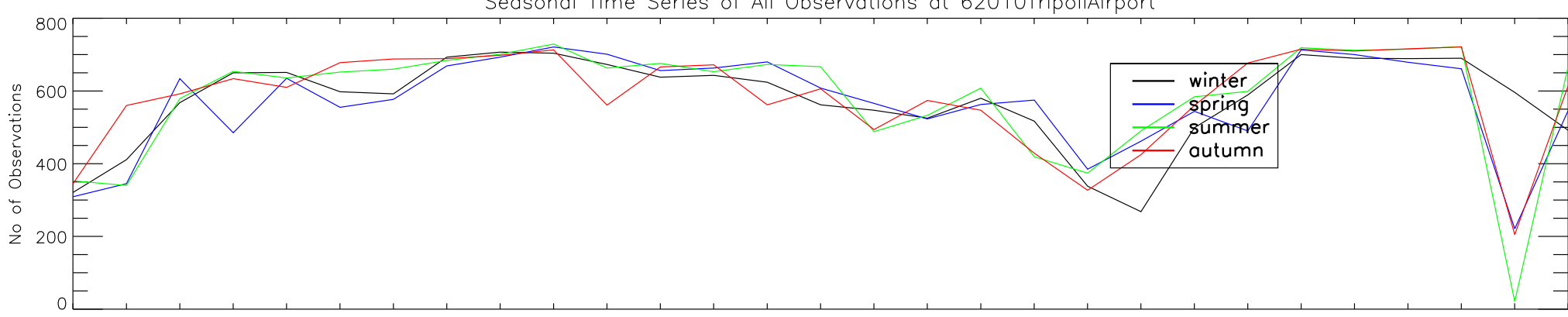

19841985198619871988198919901991199219931994199519961997199819992000200120022003200420052006200720082009201020112012

Seasonal Time Series of Dust Observations at 62010TripoliAirport

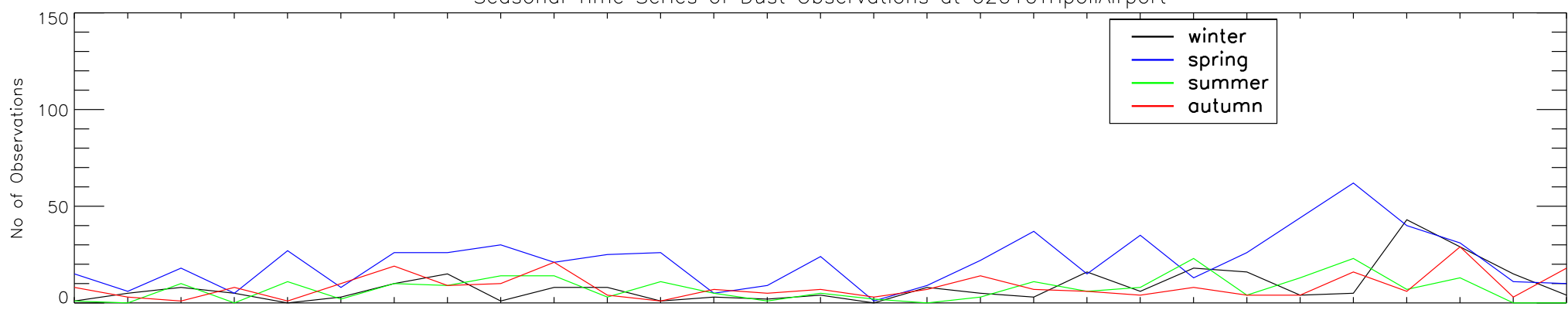

19841985198619871988198919901991199219931994199519961997199819992000200120022003200420052006200720082009201020112012 Time 
Time Series of All Observations at 62055Agedabia

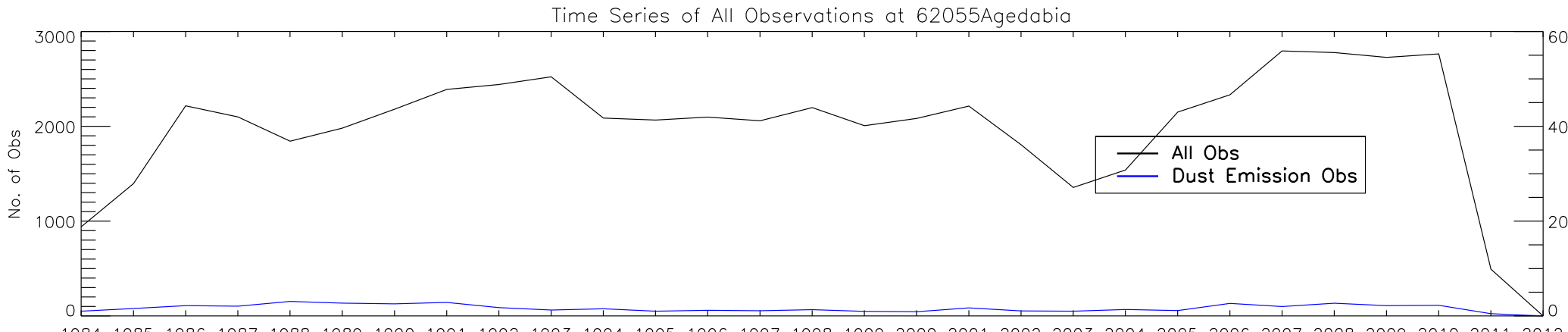

19841985198619871988198919901991199219931994199519961997199819992000200120022003200420052006200720082009201020112012 Time

Seasonal Time Series of All Observations at 62055Agedabia

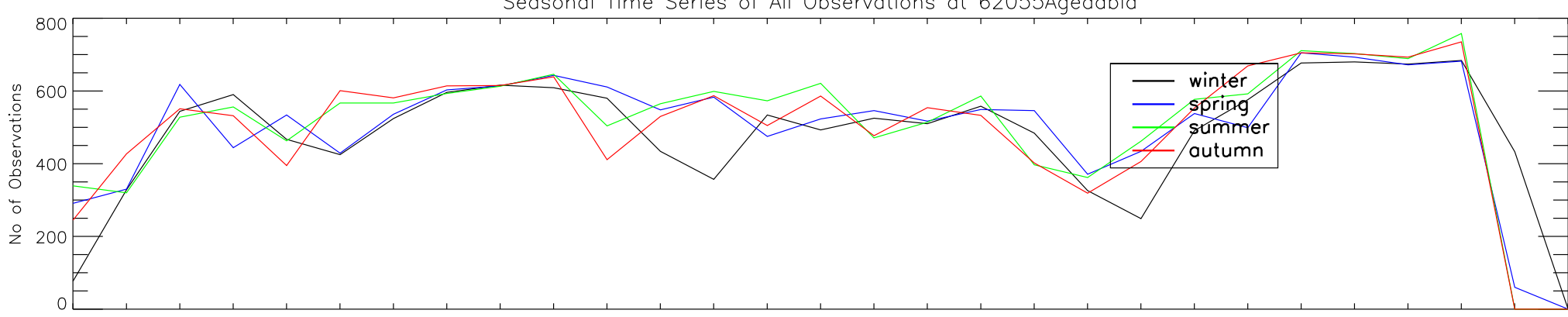

19841985198619871988198919901991199219931994199519961997199819992000200120022003200420052006200720082009201020112012

Seasonal Time Series of Dust Observations at 62055Agedabia

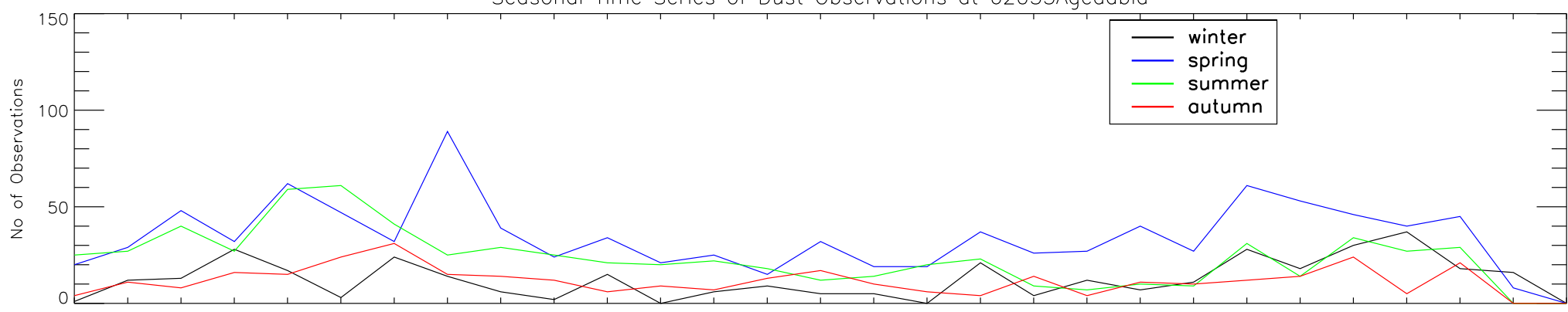

19841985198619871988198919901991199219931994199519961997199819992000200120022003200420052006200720082009201020112012 Time 
Time Series of All Observations at 62124Sebha

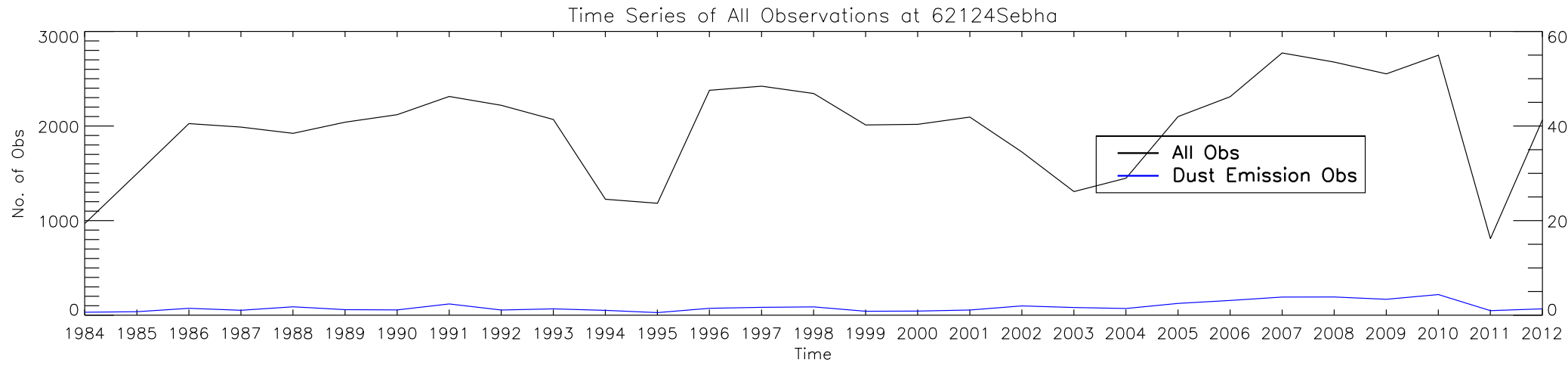

Seasonal Time Series of All Observations at 62124Sebha

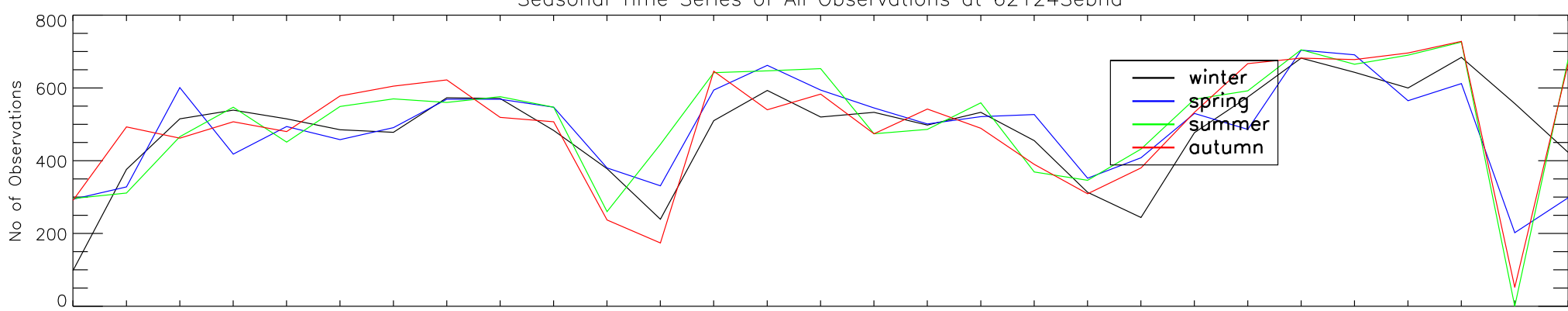

19841985198619871988198919901991199219931994199519961997199819992000200120022003200420052006200720082009201020112012

Seasonal Time Series of Dust Observations at 62124Sebha

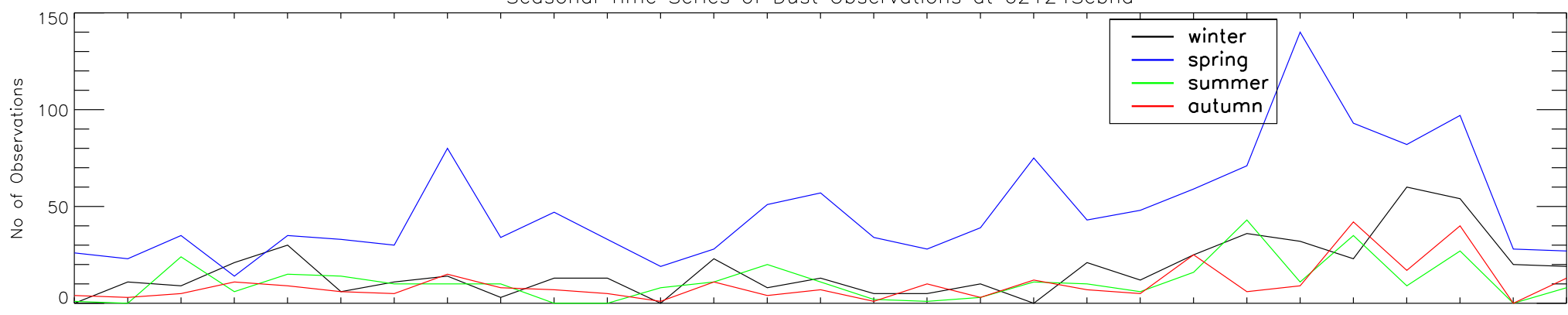

19841985198619871988198919901991199219931994199519961997199819992000200120022003200420052006200720082009201020112012 Time 
Time Series of All Observations at $62131 \mathrm{Hon}$

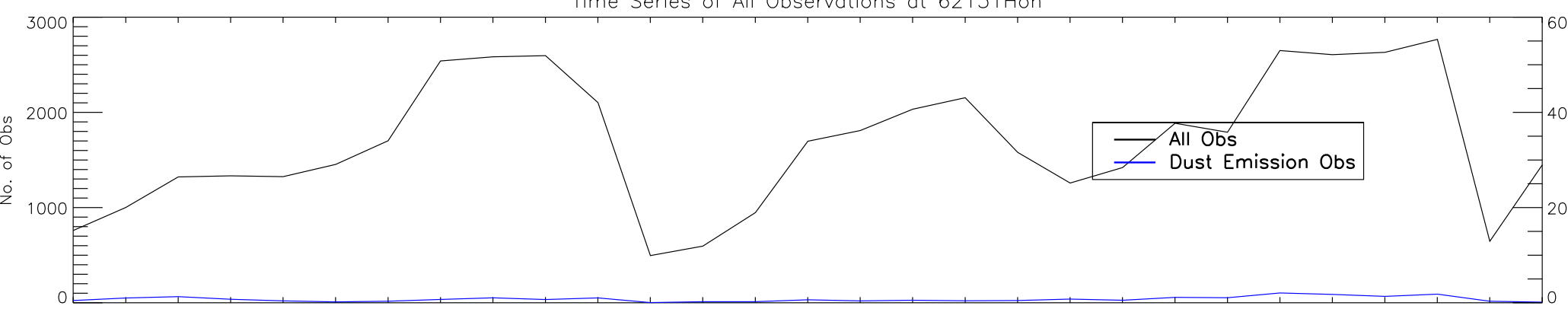

19841985198619871988198919901991199219931994199519961997199819992000200120022003200420052006200720082009201020112012 Time

Seasonal Time Series of All Observations at $62131 \mathrm{Hon}$

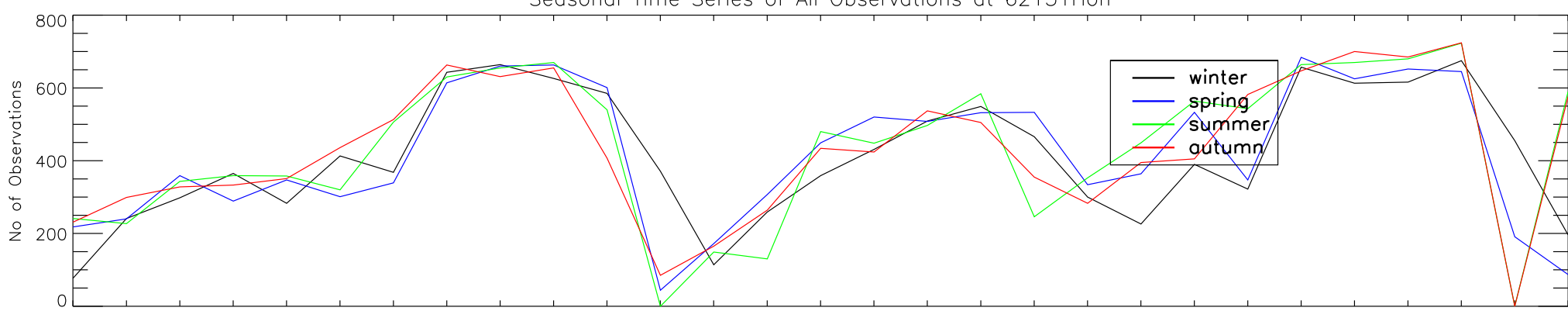

19841985198619871988198919901991199219931994199519961997199819992000200120022003200420052006200720082009201020112012

Seasonal Time Series of Dust Observations at 62131Hon

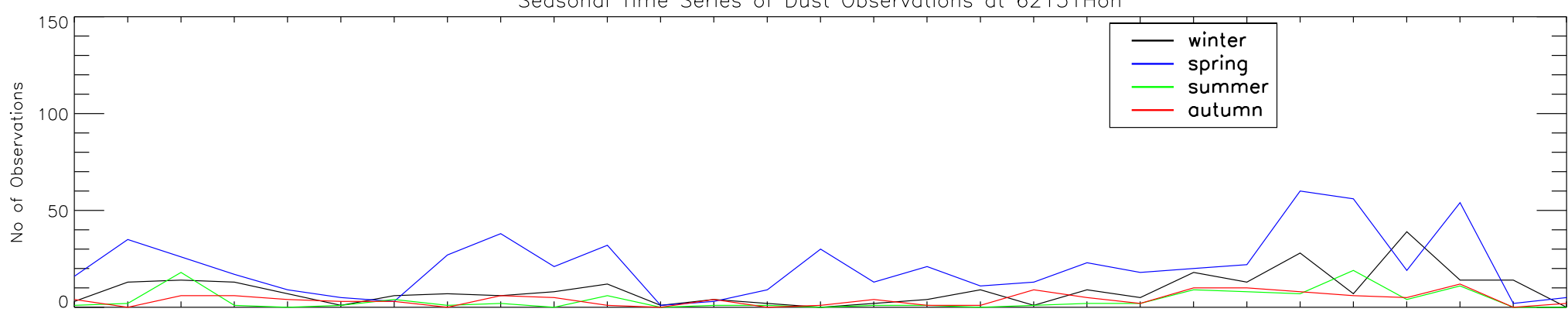

19841985198619871988198919901991199219931994199519961997199819992000200120022003200420052006200720082009201020112012 Time 


\section{Time Series of All Observations at 62212Ghat}

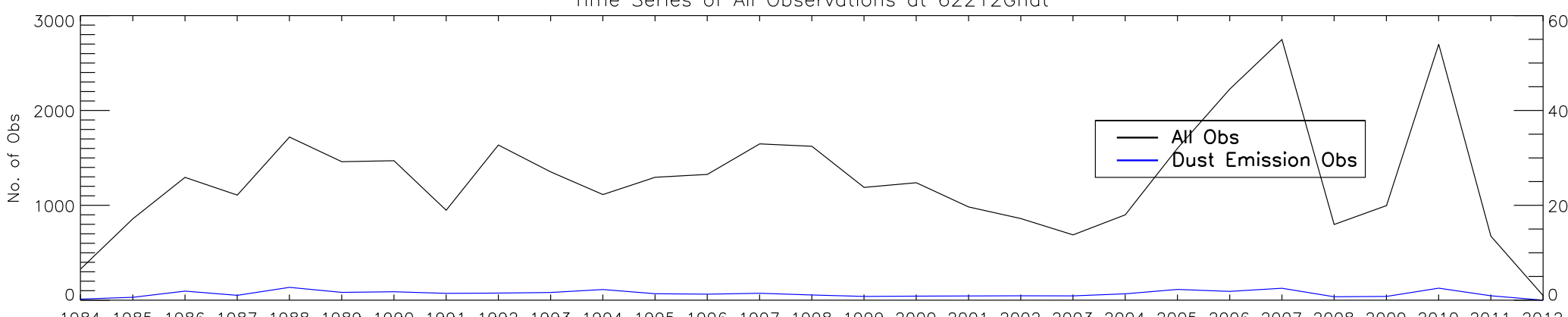

19841985198619871988198919901991199219931994199519961997199819992000200120022003200420052006200720082009201020112012 Time

Seasonal Time Series of All Observations at 62212Ghat

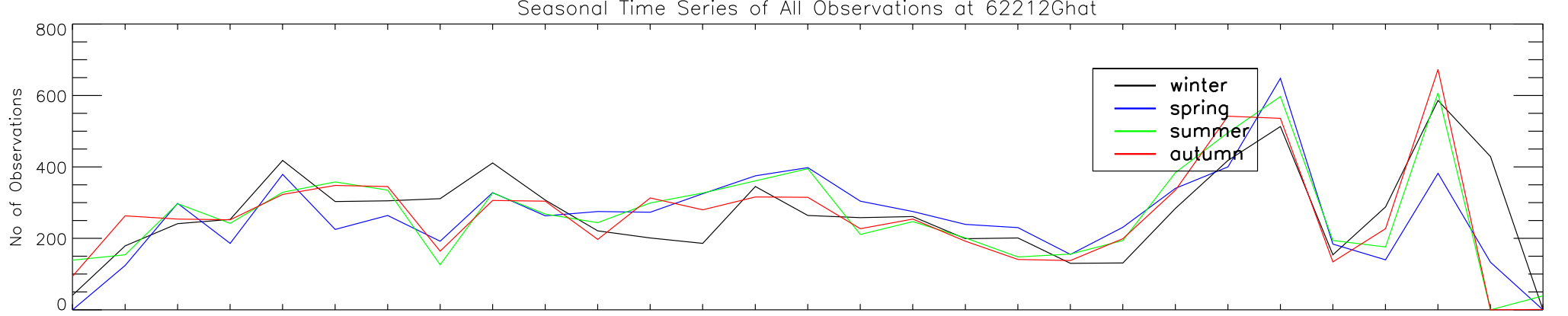

19841985198619871988198919901991199219931994199519961997199819992000200120022003200420052006200720082009201020112012

Seasonal Time Series of Dust Observations at 62212Ghat

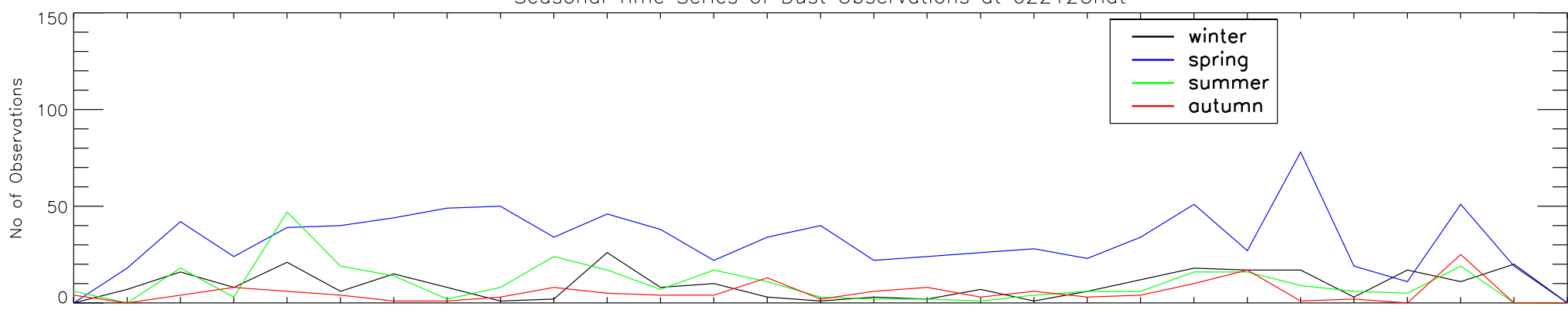

19841985198619871988198919901991199219931994199519961997199819992000200120022003200420052006200720082009201020112012 Time 


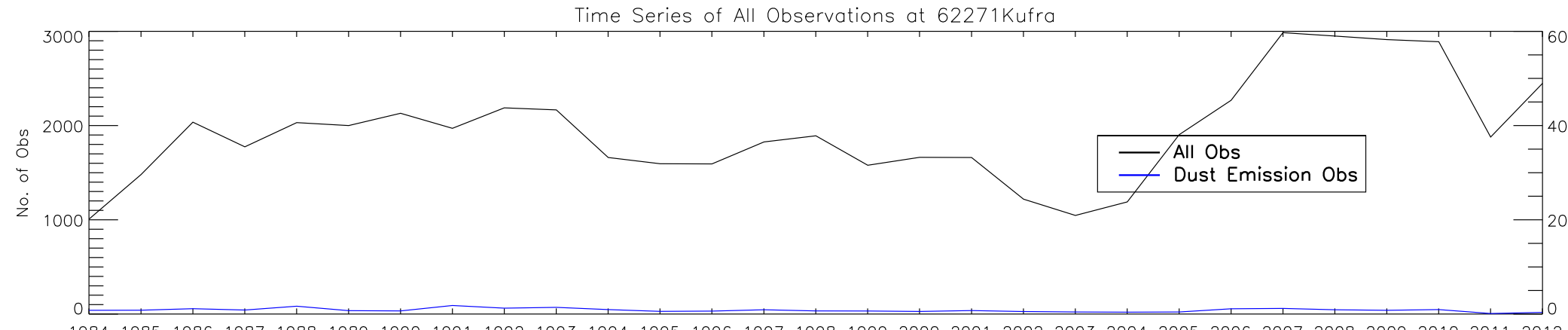

19841985198619871988198919901991199219931994199519961997199819992000200120022003200420052006200720082009201020112012 Time

Seasonal Time Series of All Observations at 62271Kufra

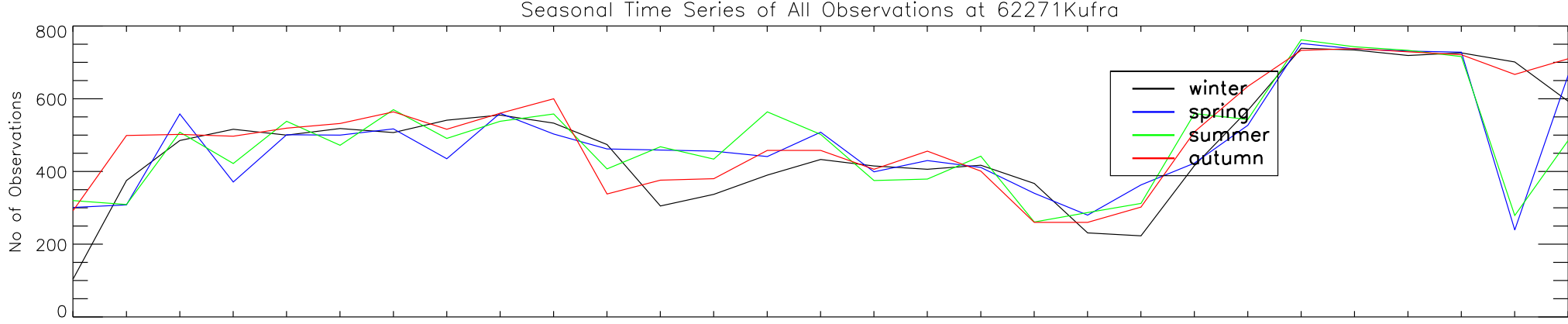

19841985198619871988198919901991199219931994199519961997199819992000200120022003200420052006200720082009201020112012

Seasonal Time Series of Dust Observations at 62271Kufra

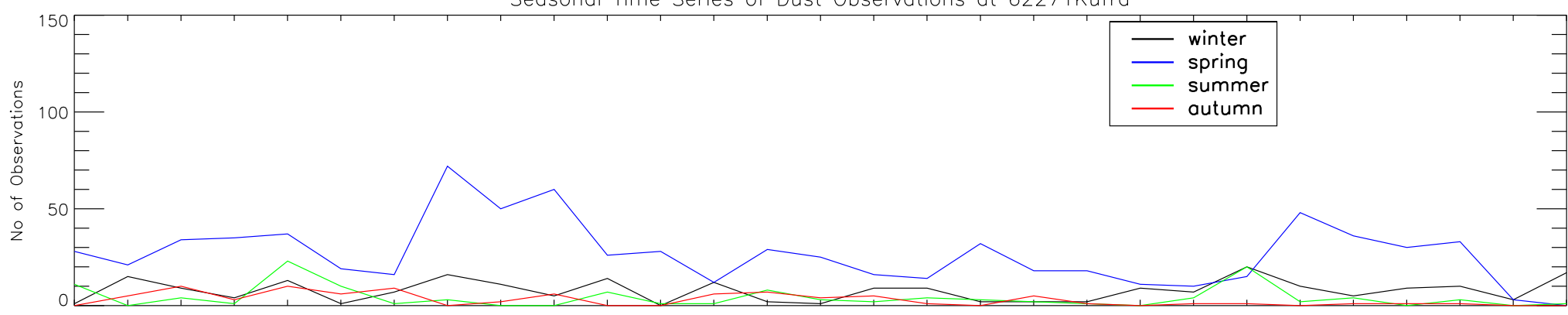

19841985198619871988198919901991199219931994199519961997199819992000200120022003200420052006200720082009201020112012 Time 
 \\ $3000+\frac{1}{2}$ Time Series of All Observations at 62306MersaMatruh}

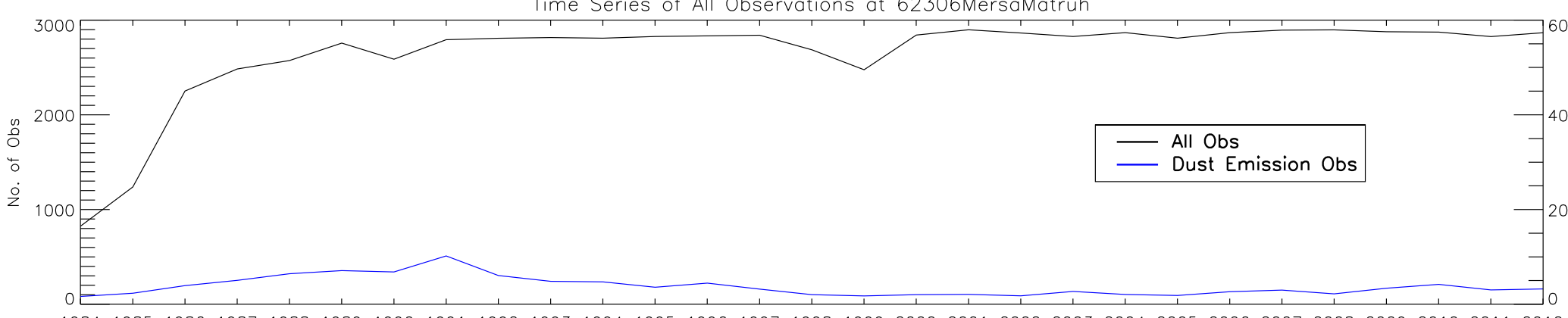

19841985198619871988198919901991199219931994199519961997199819992000200120022003200420052006200720082009201020112012 Time

Seasonal Time Series of All Observations at 62306MersaMatruh

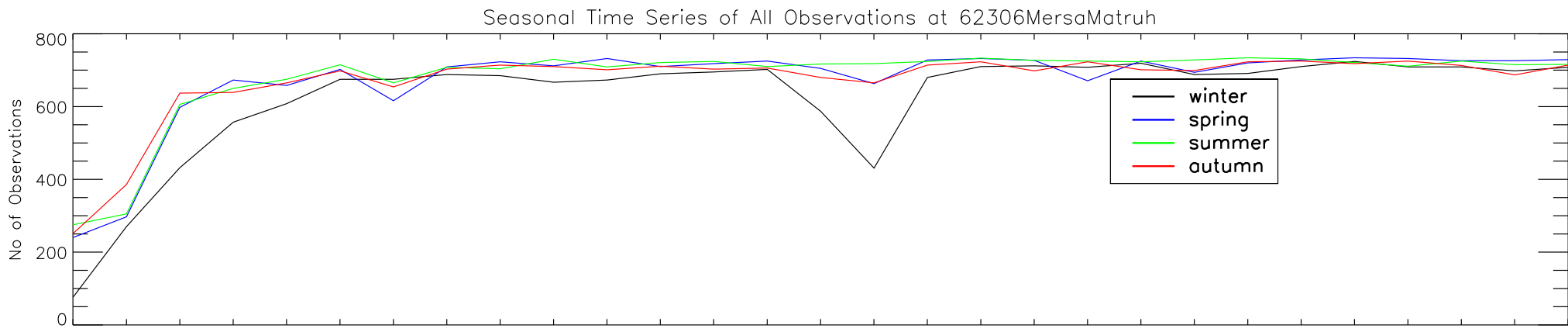

19841985198619871988198919901991199219931994199519961997199819992000200120022003200420052006200720082009201020112012

Seasonal Time Series of Dust Observations at 62306MersaMatruh

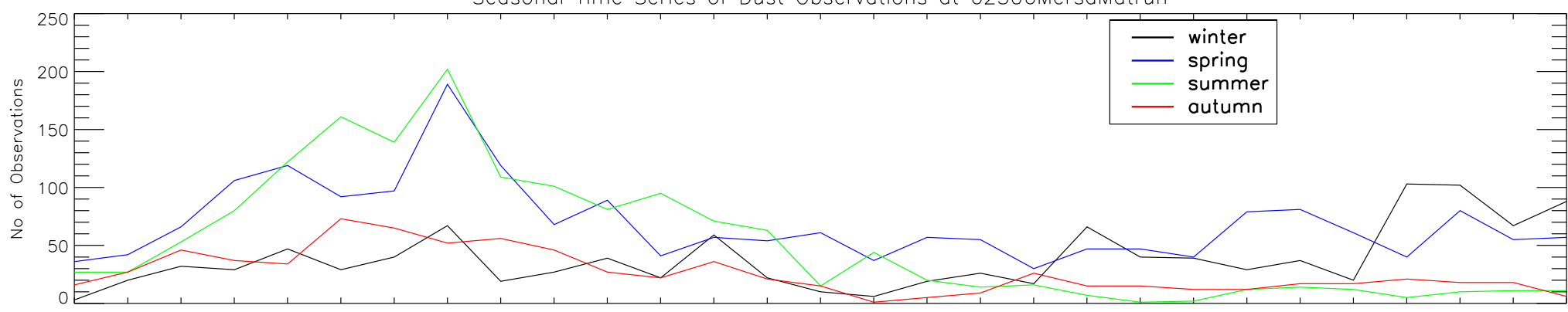

19841985198619871988198919901991199219931994199519961997199819992000200120022003200420052006200720082009201020112012 Time 
Time Series of All Observations at 62309Dabaa

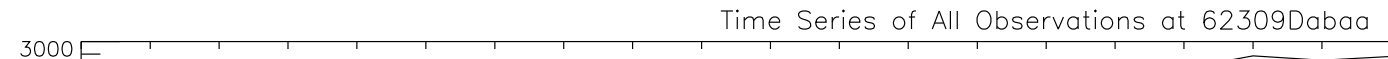

All Obs

Dust Emission Obs

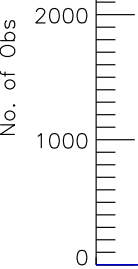

198419851986198719881989199019911992199319941995199619971998

Seasonal Time Series of All Observations at 62309Dabaa

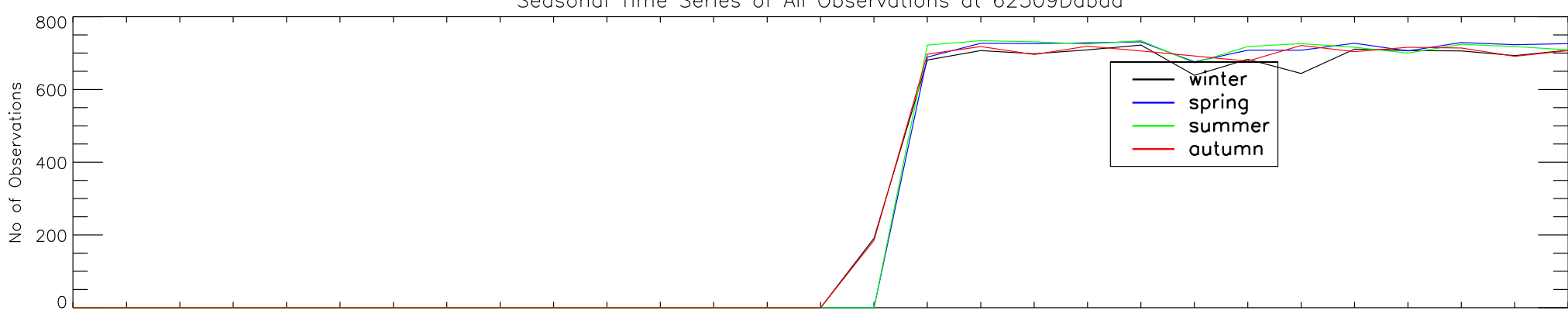

19841985198619871988198919901991199219931994199519961997199819992000200120022003200420052006200720082009201020112012

Seasonal Time Series of Dust Observations at 62309Dabad

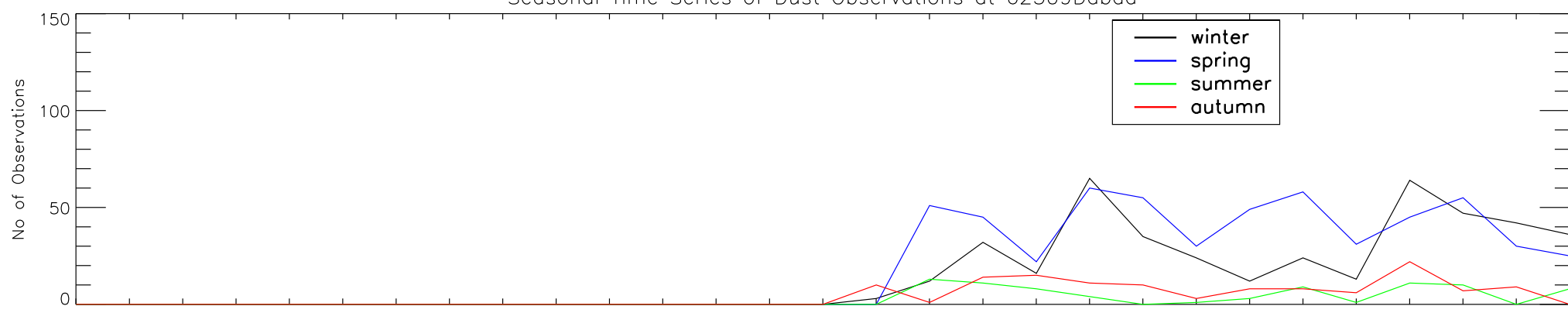

19841985198619871988198919901991199219931994199519961997199819992000200120022003200420052006200720082009201020112012 
 \\ 3000}

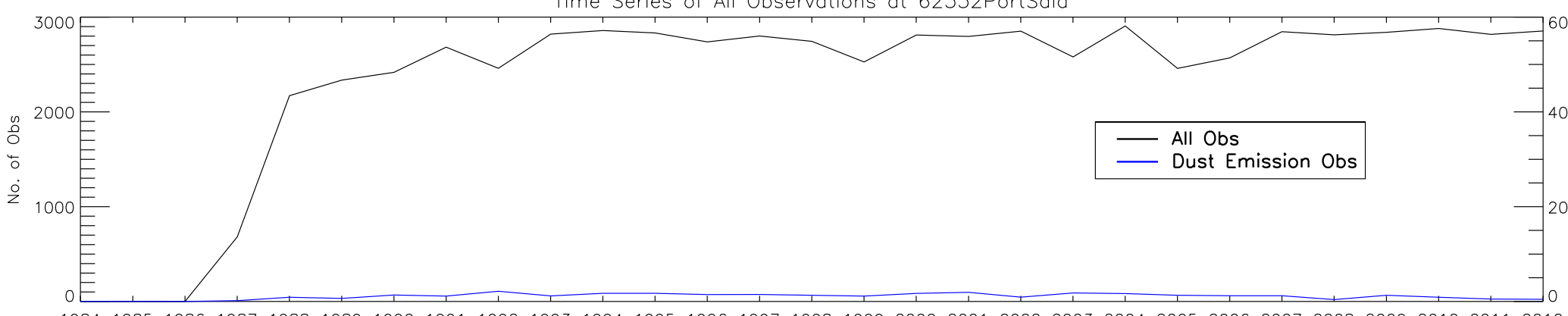

19841985198619871988198919901991199219931994199519961997199819992000200120022003200420052006200720082009201020112012 Time

Seasonal Time Series of All Observations at 62332PortSaid

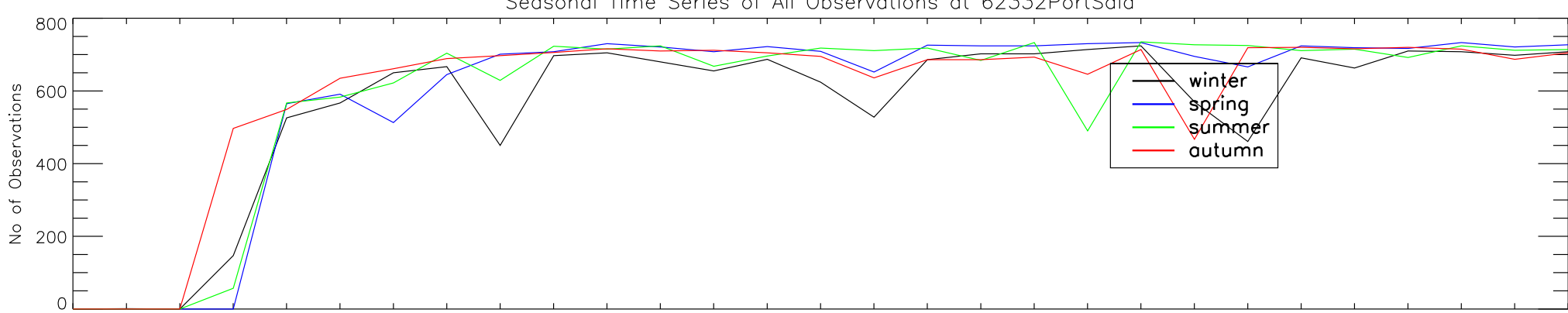

19841985198619871988198919901991199219931994199519961997199819992000200120022003200420052006200720082009201020112012

Seasonal Time Series of Dust Observations at 62332PortSaid

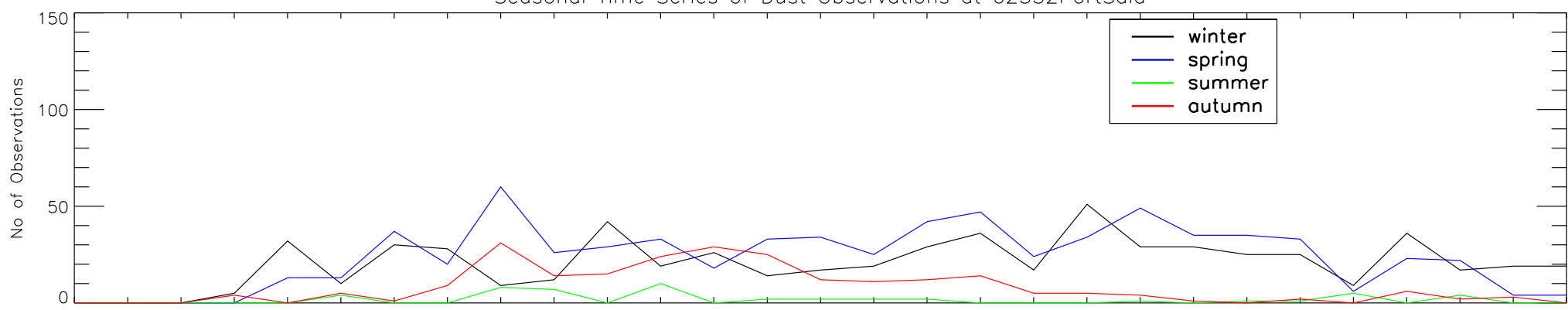

19841985198619871988198919901991199219931994199519961997199819992000200120022003200420052006200720082009201020112012 Time 
Time Series of All Observations at 62337E|Arish

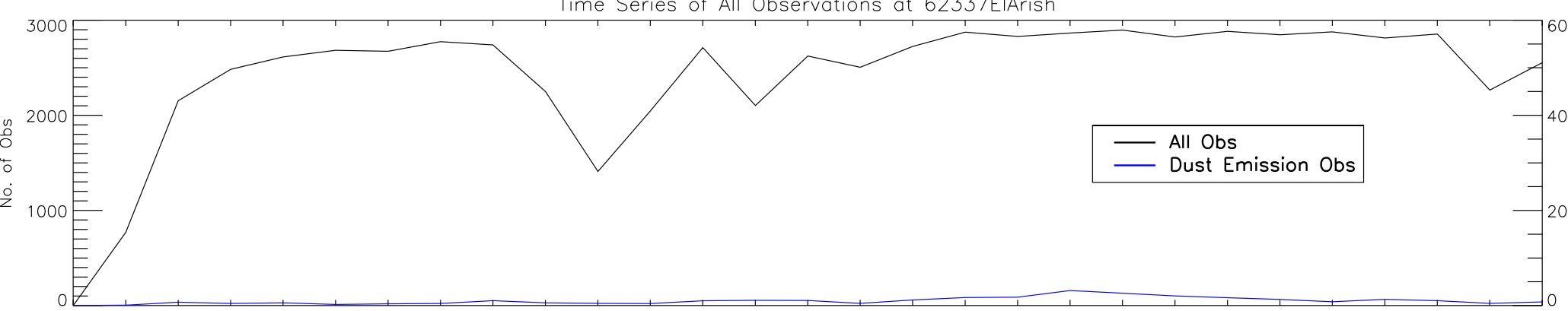

19841985198619871988198919901991199219931994199519961997199819992000200120022003200420052006200720082009201020112012 Time

Seasonal Time Series of All Observations at 62337ElArish

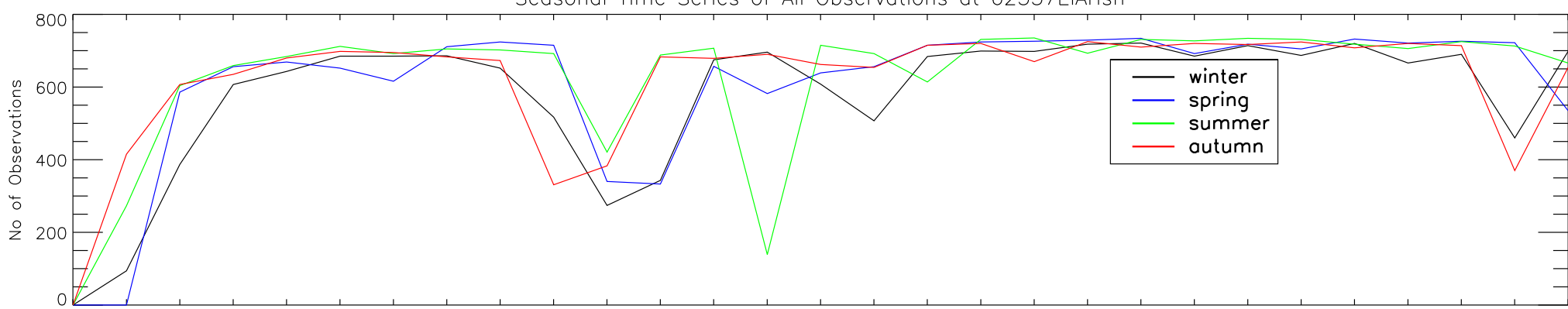

19841985198619871988198919901991199219931994199519961997199819992000200120022003200420052006200720082009201020112012

Seasonal Time Series of Dust Observations at 62337ElArish

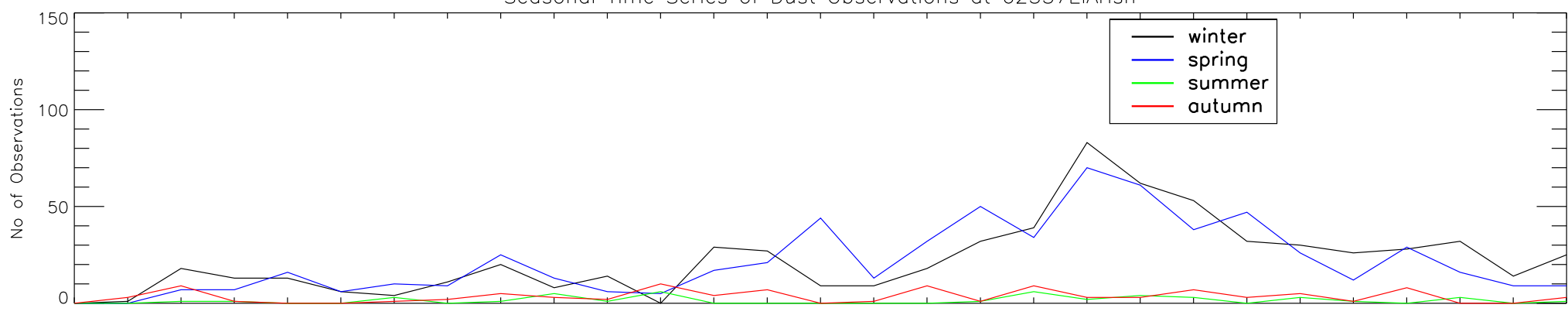

19841985198619871988198919901991199219931994199519961997199819992000200120022003200420052006200720082009201020112012 Time 
 \\ $3000+T_{1}$ Time Series of All Observations at 62387Minya}

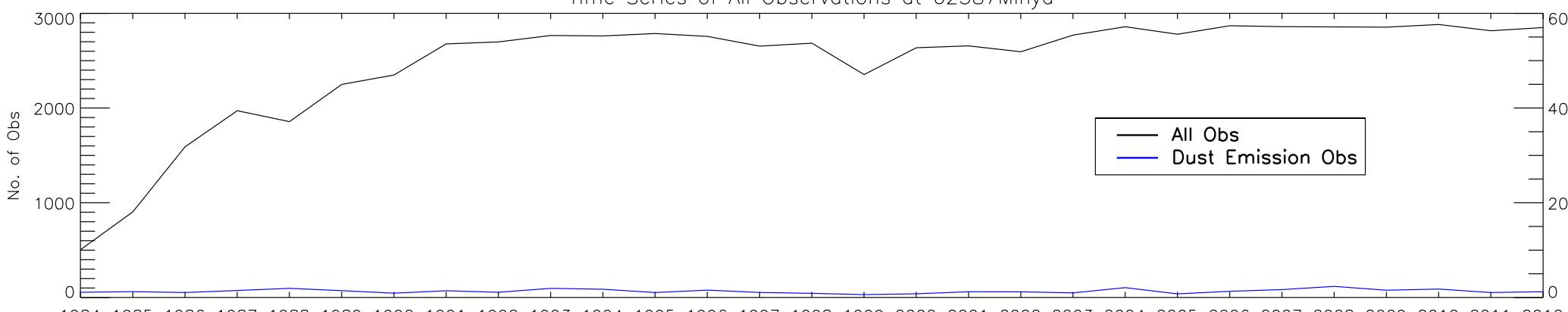

19841985198619871988198919901991199219931994199519961997199819992000200120022003200420052006200720082009201020112012 Time

Seasonal Time Series of All Observations at 62387Minya

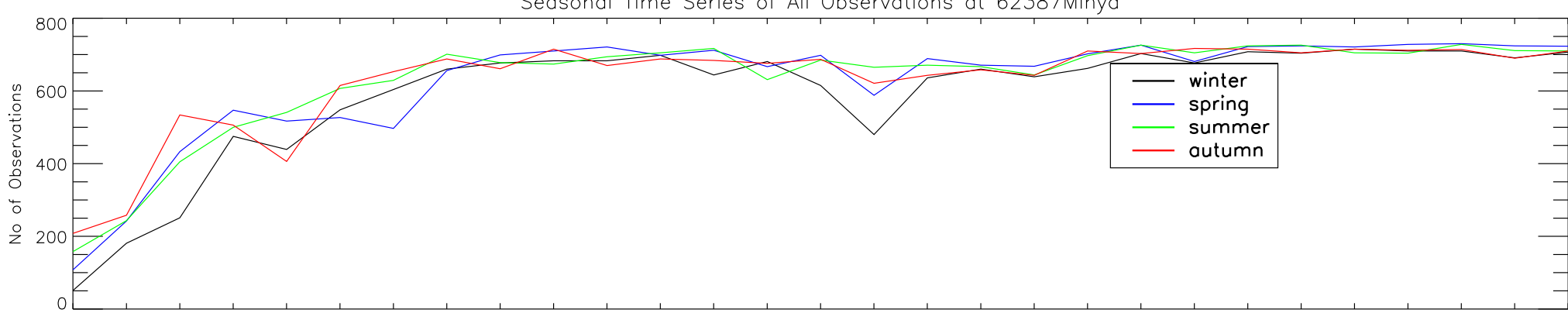

19841985198619871988198919901991199219931994199519961997199819992000200120022003200420052006200720082009201020112012

Seasonal Time Series of Dust Observations at 62387Minya

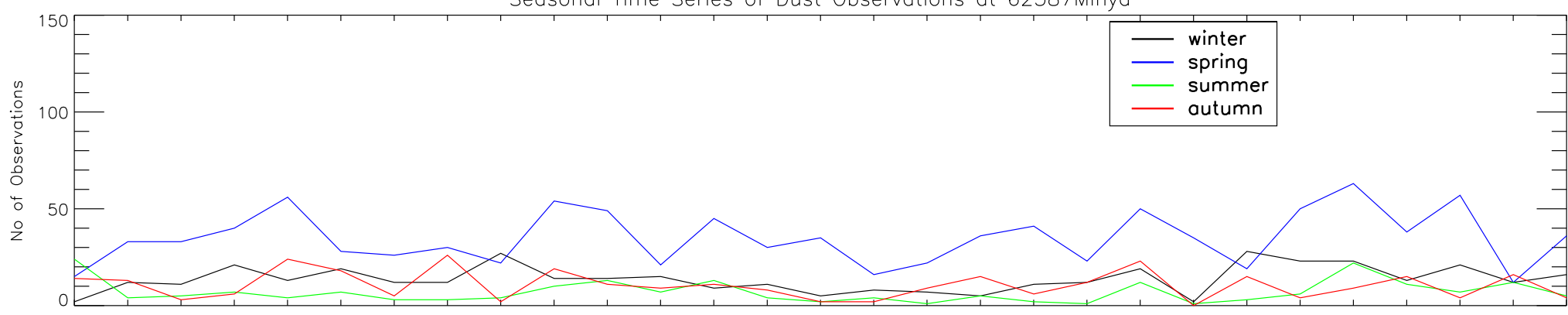

19841985198619871988198919901991199219931994199519961997199819992000200120022003200420052006200720082009201020112012 Time 
Time Series of All Observations at 62393Asyut

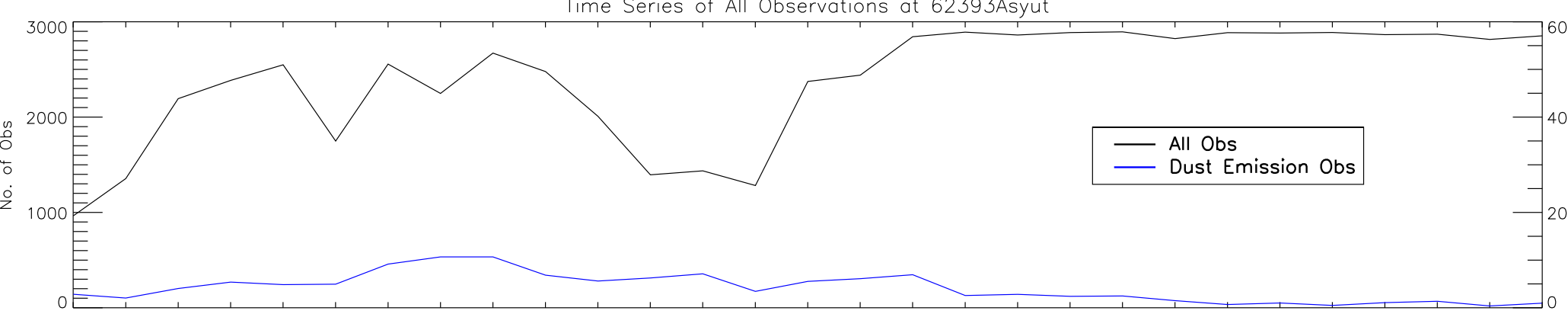

19841985198619871988198919901991199219931994199519961997199819992000200120022003200420052006200720082009201020112012 Time

Seasonal Time Series of All Observations at 62393Asyut

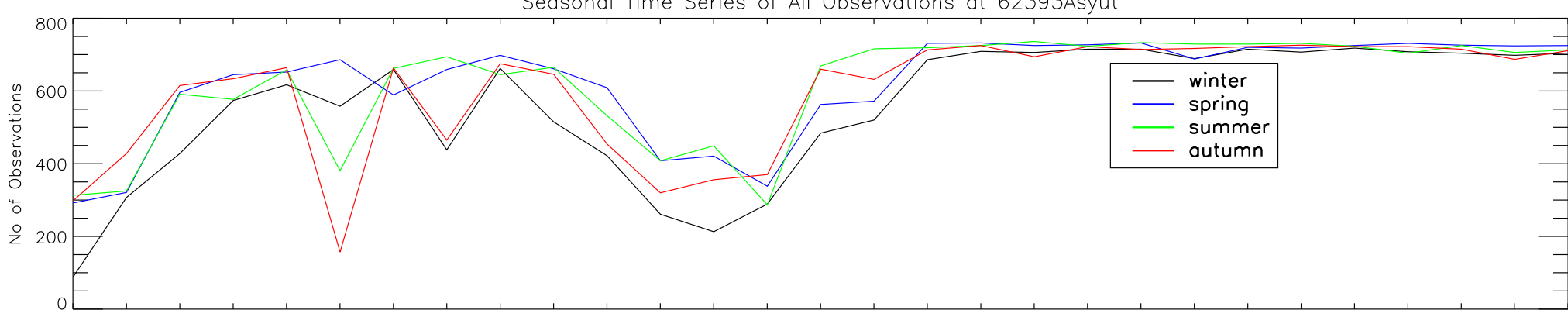

19841985198619871988198919901991199219931994199519961997199819992000200120022003200420052006200720082009201020112012

Seasonal Time Series of Dust Observations at 62393Asyut

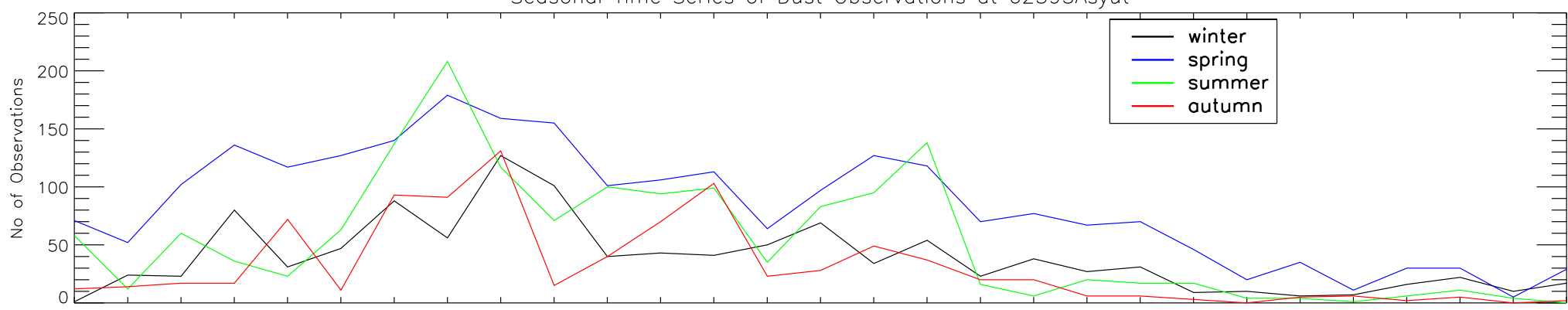

19841985198619871988198919901991199219931994199519961997199819992000200120022003200420052006200720082009201020112012 Time 


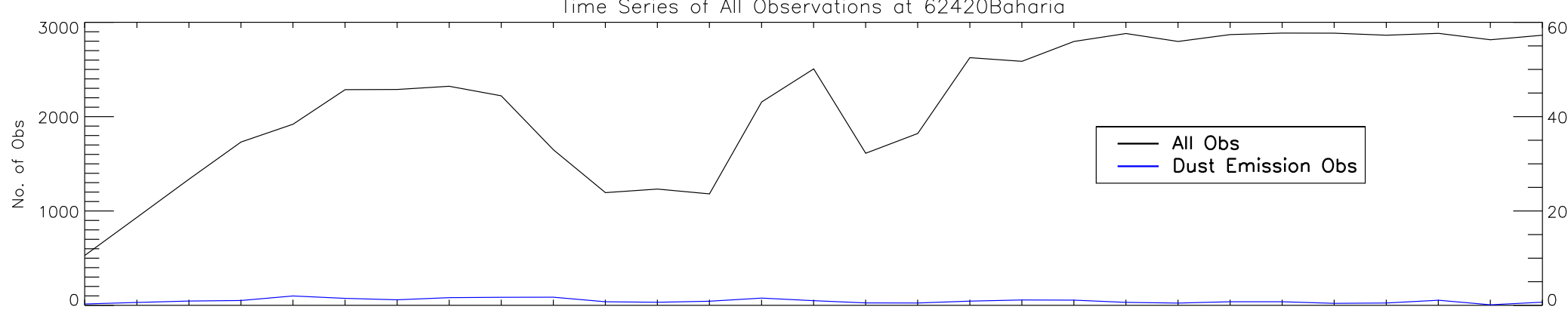

19841985198619871988198919901991199219931994199519961997199819992000200120022003200420052006200720082009201020112012 Time

Seasonal Time Series of All Observations at 62420Baharia

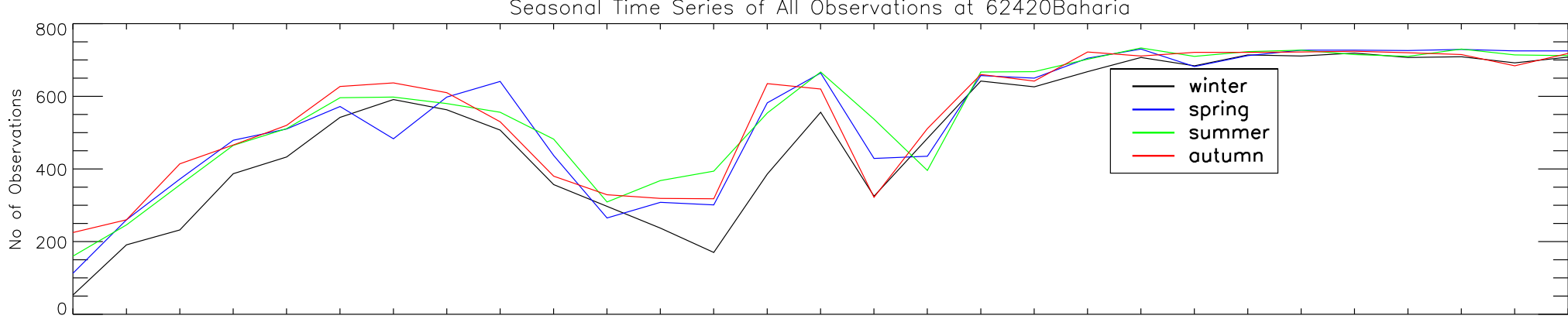

19841985198619871988198919901991199219931994199519961997199819992000200120022003200420052006200720082009201020112012

Seasonal Time Series of Dust Observations at 62420Baharia

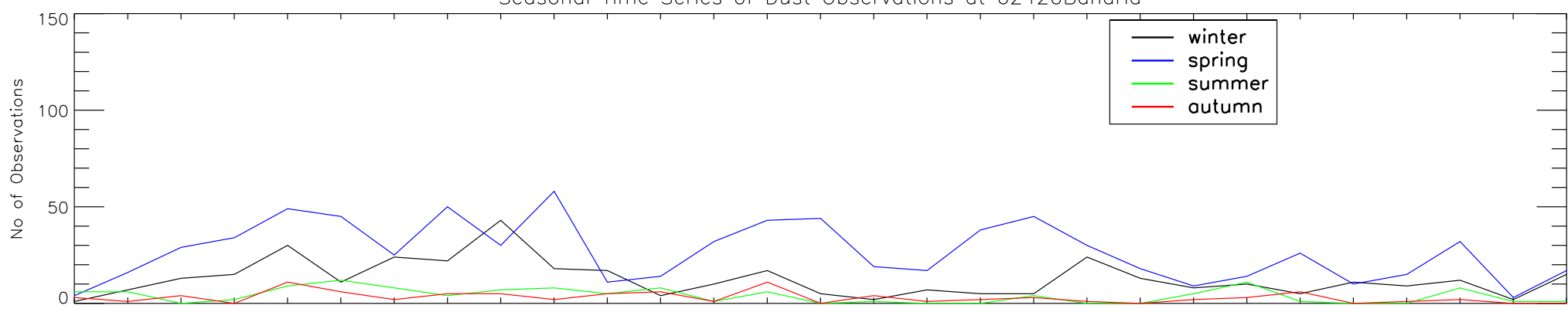

19841985198619871988198919901991199219931994199519961997199819992000200120022003200420052006200720082009201020112012 Time 

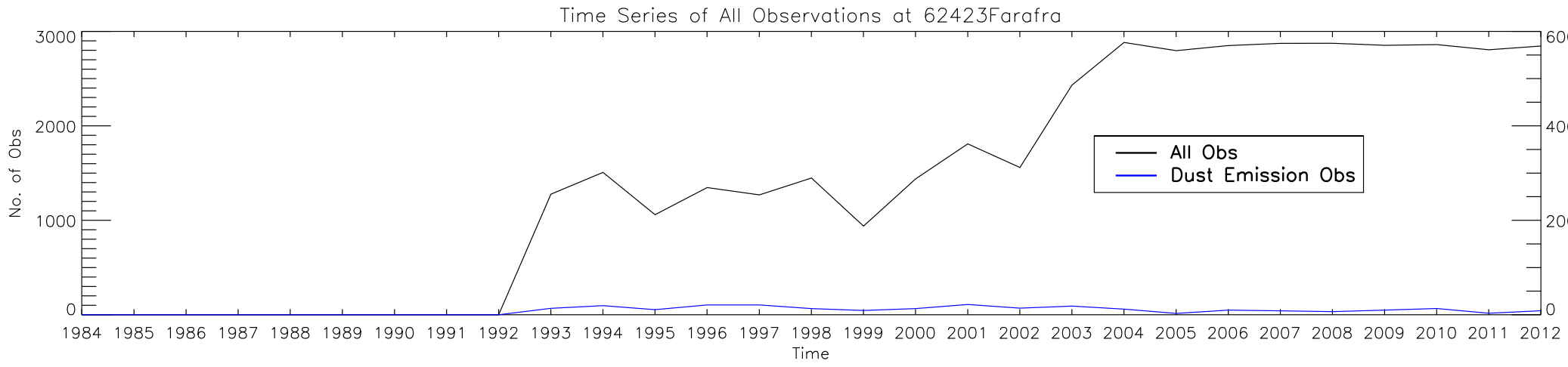

Seasonal Time Series of All Observations at 62423Farafra

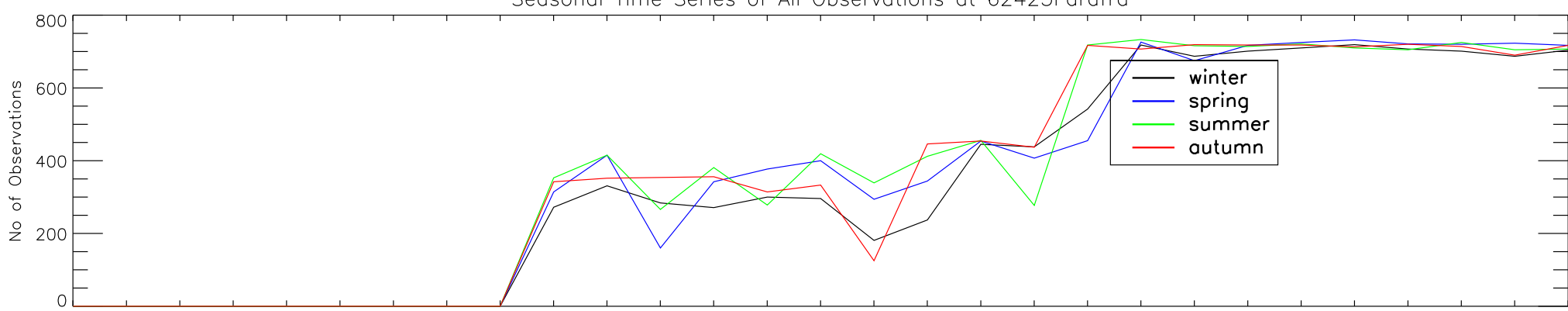

19841985198619871988198919901991199219931994199519961997199819992000200120022003200420052006200720082009201020112012

Seasonal Time Series of Dust Observations at 62423Farafra

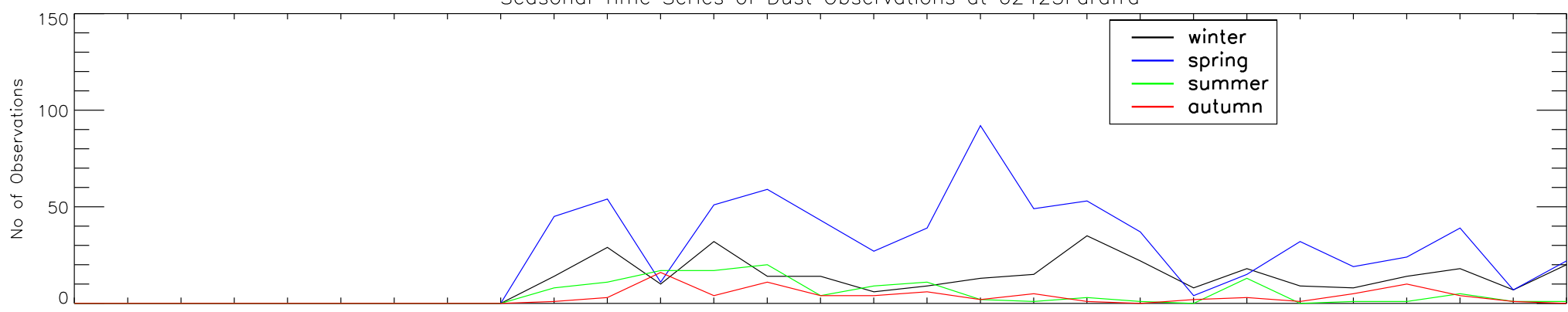

19841985198619871988198919901991199219931994199519961997199819992000200120022003200420052006200720082009201020112012 Time 
 \\ 3000}

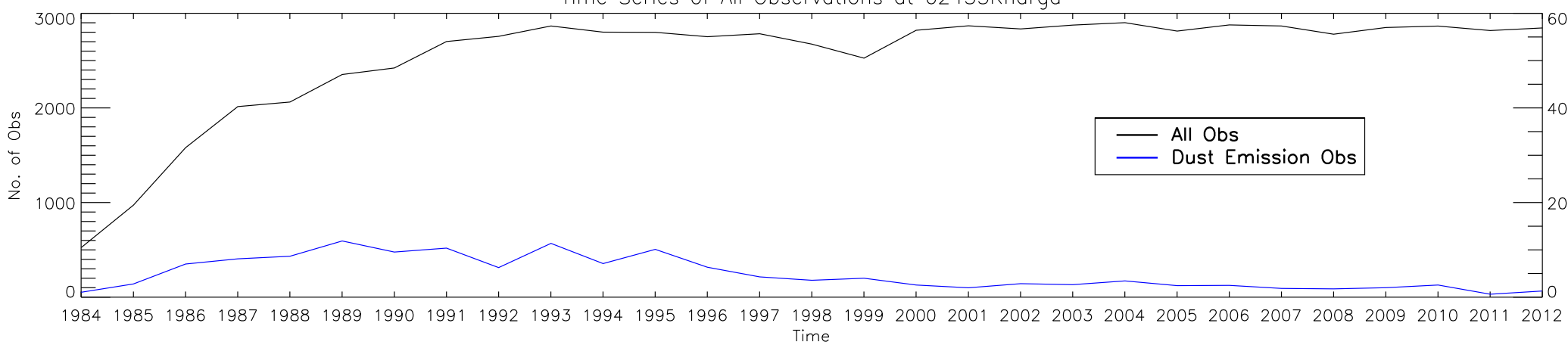

Seasonal Time Series of All Observations at 62435Kharga

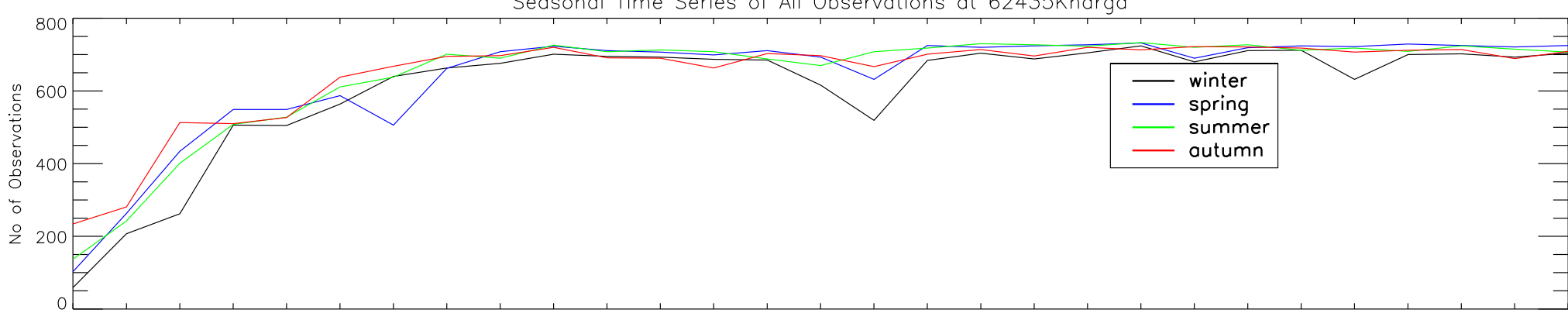

19841985198619871988198919901991199219931994199519961997199819992000200120022003200420052006200720082009201020112012

Seasonal Time Series of Dust Observations at 62435Kharga

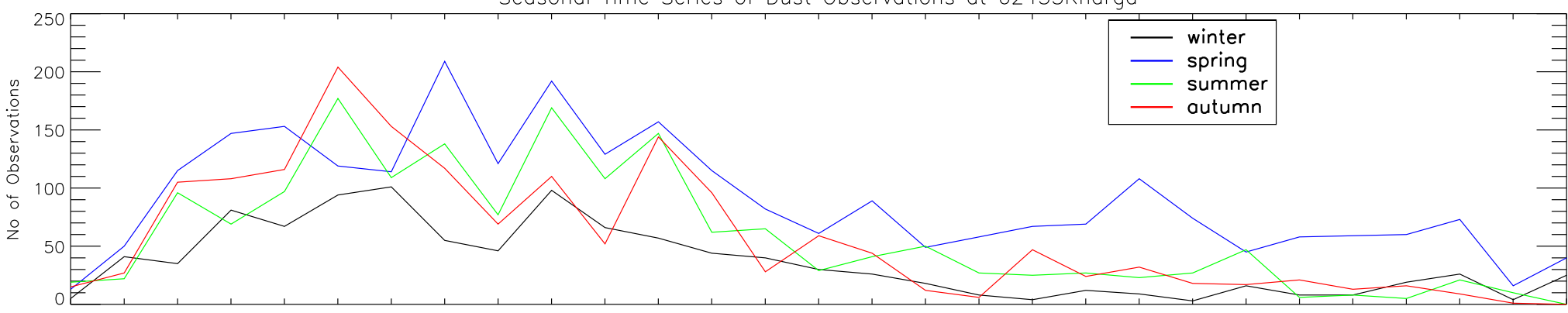

19841985198619871988198919901991199219931994199519961997199819992000200120022003200420052006200720082009201020112012 Time 
Time Series of All Observations at 62600 WadiHalfa

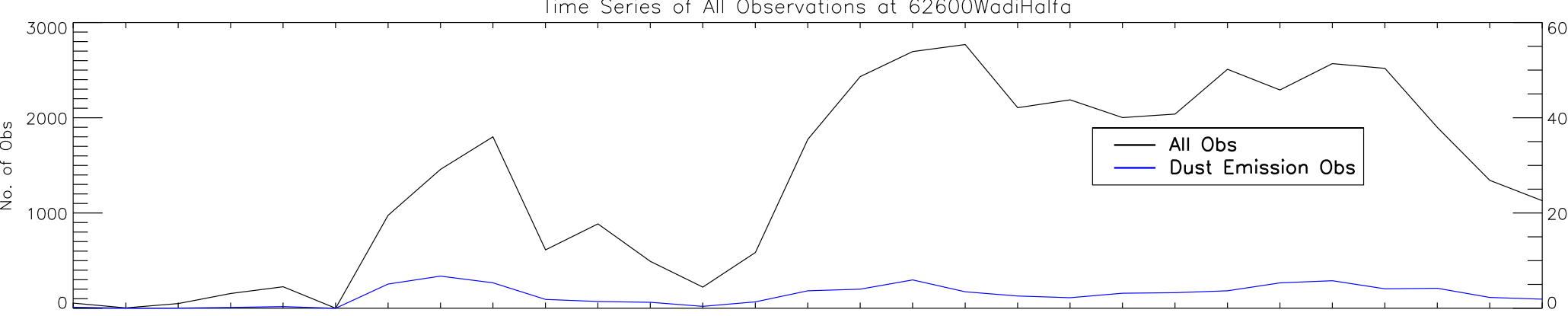

19841985198619871988198919901991199219931994199519961997199819992000200120022003200420052006200720082009201020112012 Time

Seasonal Time Series of All Observations at 62600WadiHalfa

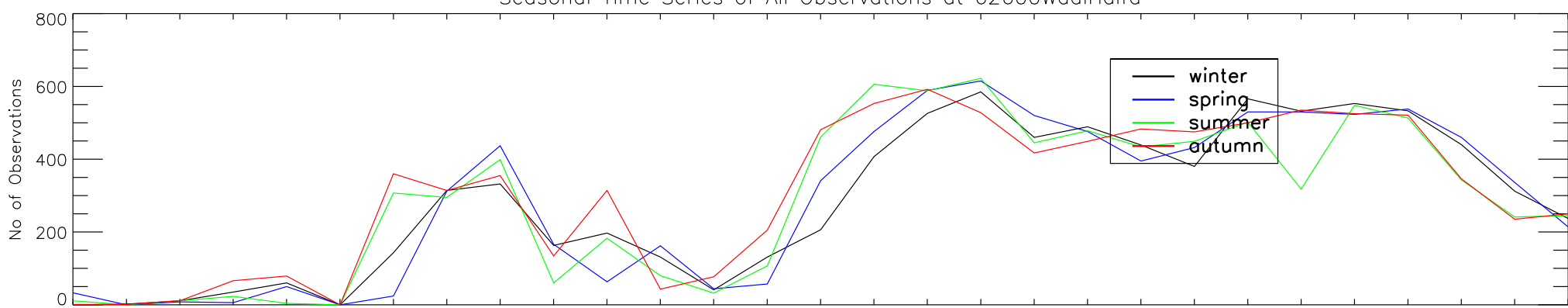

19841985198619871988198919901991199219931994199519961997199819992000200120022003200420052006200720082009201020112012

Seasonal Time Series of Dust Observations at 62600WadiHalfa

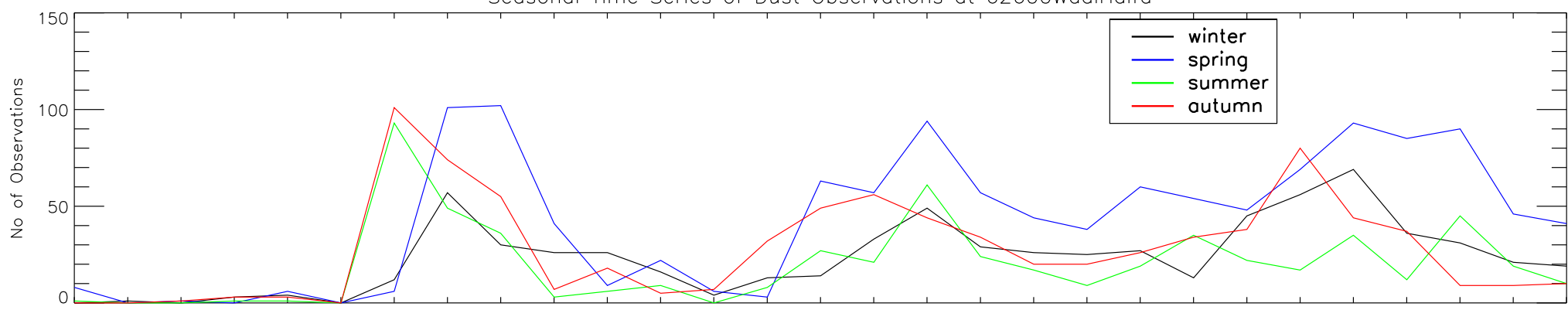

19841985198619871988198919901991199219931994199519961997199819992000200120022003200420052006200720082009201020112012 Time 
Time Series of All Observations at 62640AbuHamed

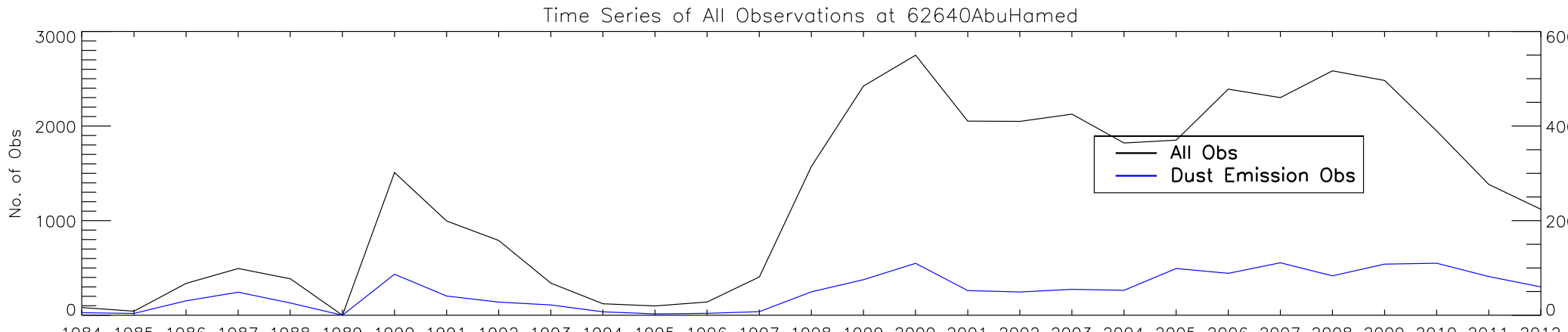

19841985198619871988198919901991199219931994199519961997199819992000200120022003200420052006200720082009201020112012

Seasonal Time Series of All Observations at 62640AbuHamed

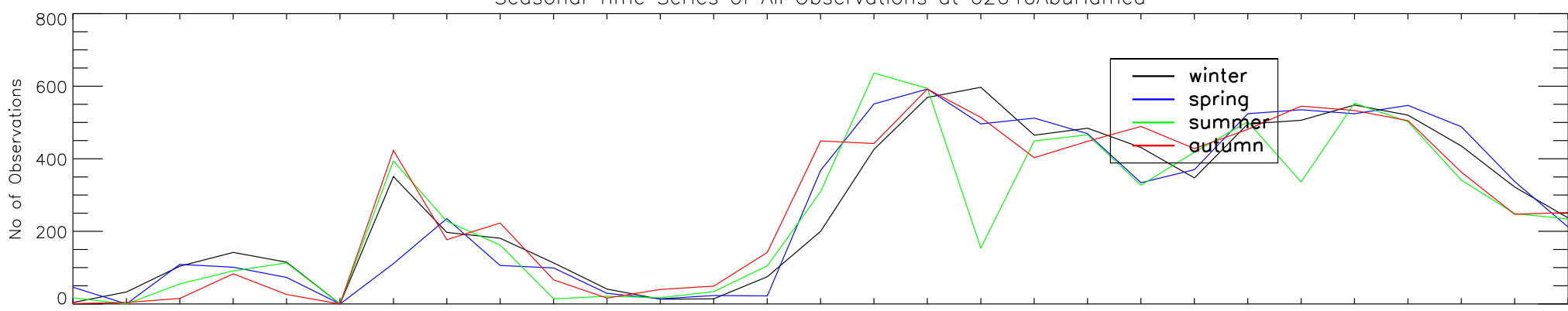

19841985198619871988198919901991199219931994199519961997199819992000200120022003200420052006200720082009201020112012

Seasonal Time Series of Dust Observations at 62640AbuHamed

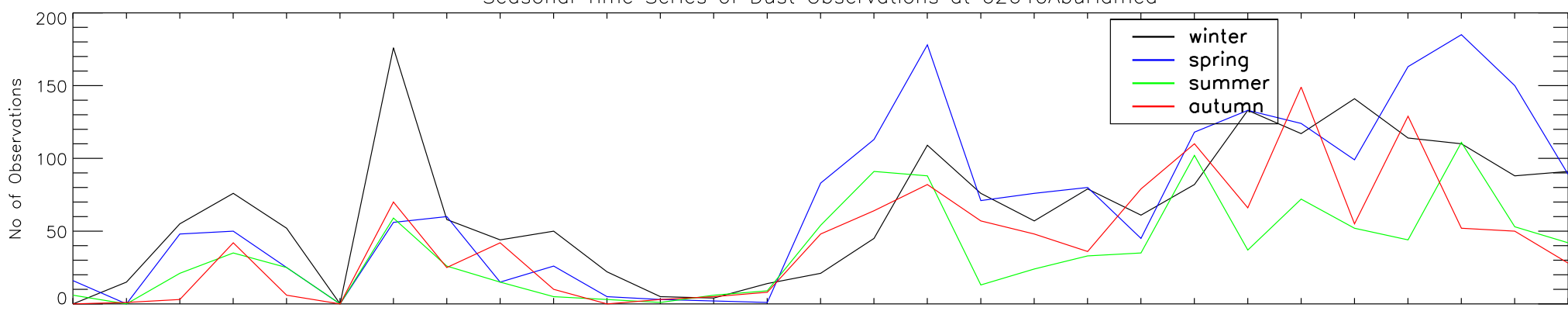

19841985198619871988198919901991199219931994199519961997199819992000200120022003200420052006200720082009201020112012 Time 
Time Series of All Observations at 62641PortSudan

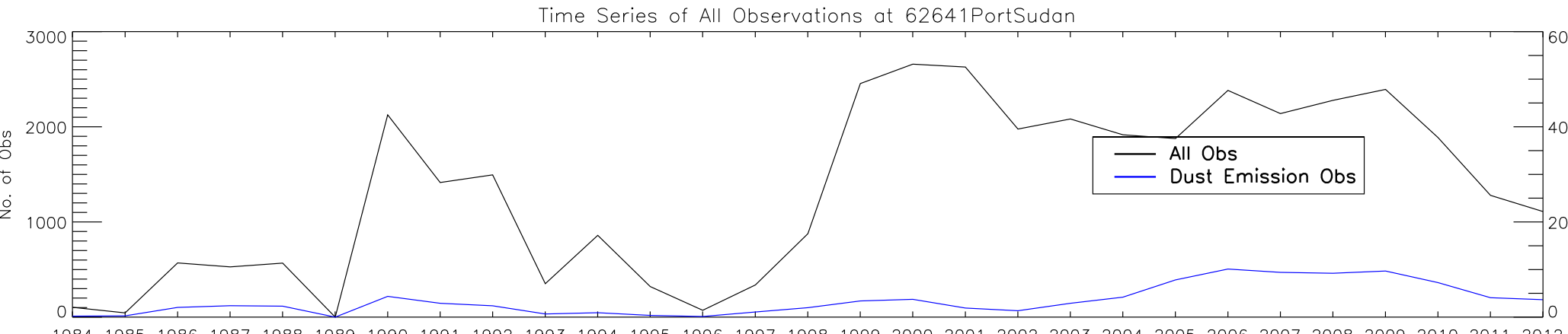

19841985198619871988198919901991199219931994199519961997199819992000200120022003200420052006200720082009201020112012 Time

Seasonal Time Series of All Observations at 62641PortSudan

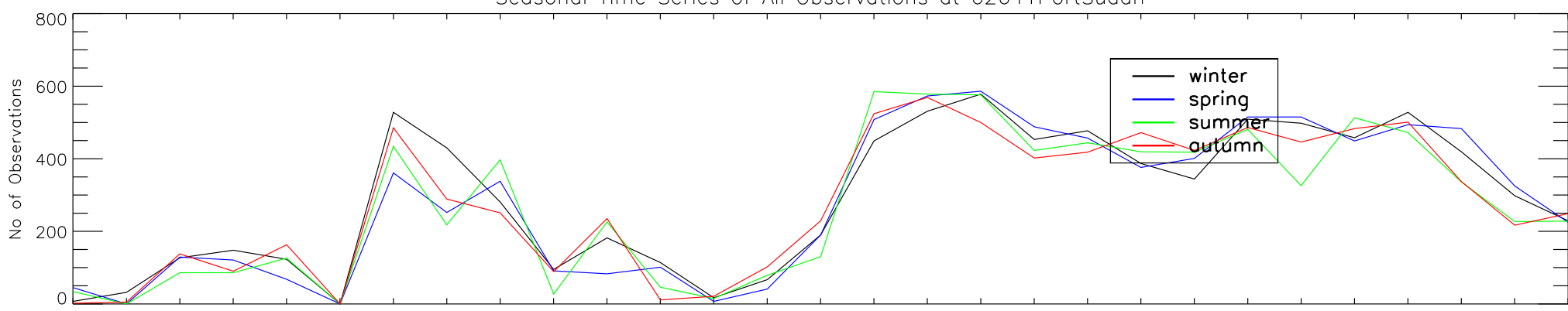

19841985198619871988198919901991199219931994199519961997199819992000200120022003200420052006200720082009201020112012

Seasonal Time Series of Dust Observations at 62641PortSudan

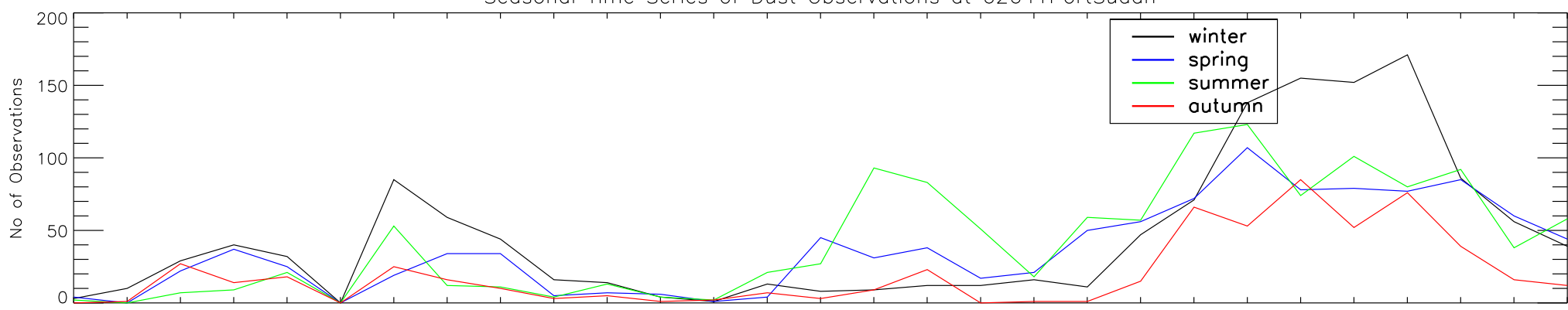

19841985198619871988198919901991199219931994199519961997199819992000200120022003200420052006200720082009201020112012 Time 
Time Series of All Observations at 62650Dongola

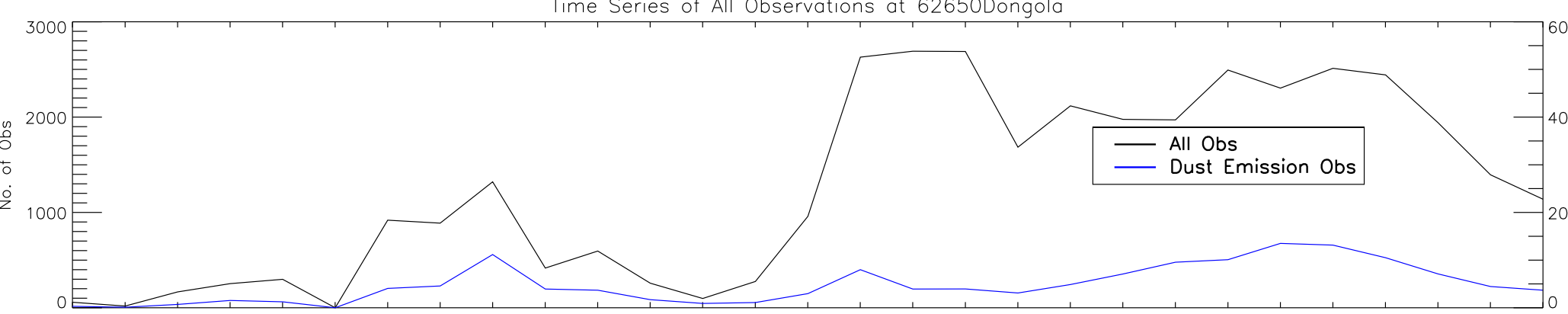

19841985198619871988198919901991199219931994199519961997199819992000200120022003200420052006200720082009201020112012 Time

Seasonal Time Series of All Observations at 62650Dongola

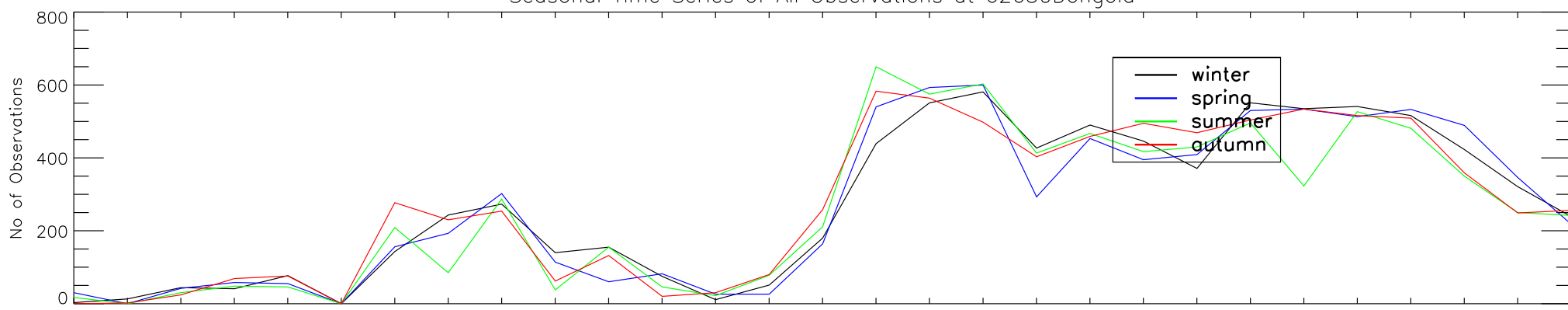

19841985198619871988198919901991199219931994199519961997199819992000200120022003200420052006200720082009201020112012

Seasonal Time Series of Dust Observations at 62650Dongola

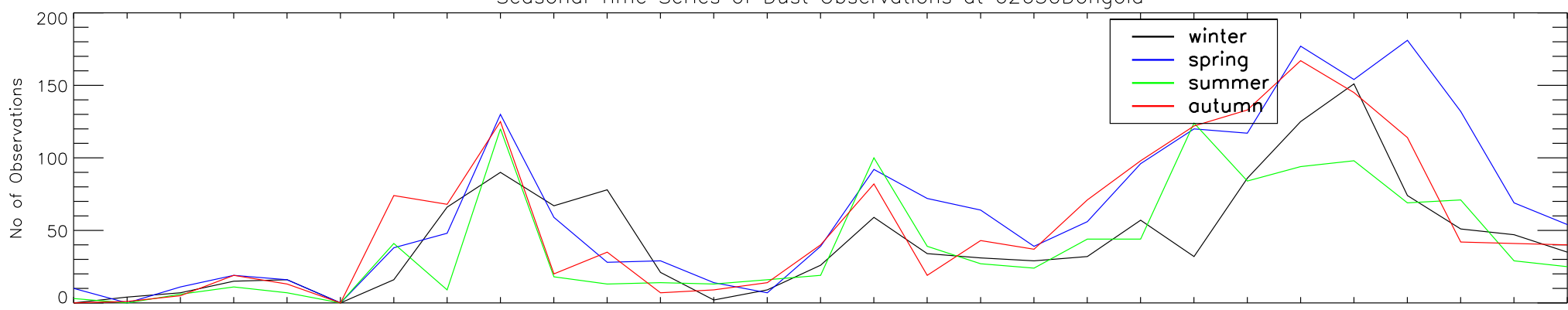

19841985198619871988198919901991199219931994199519961997199819992000200120022003200420052006200720082009201020112012 Time 
Time Series of All Observations at 62660Karima

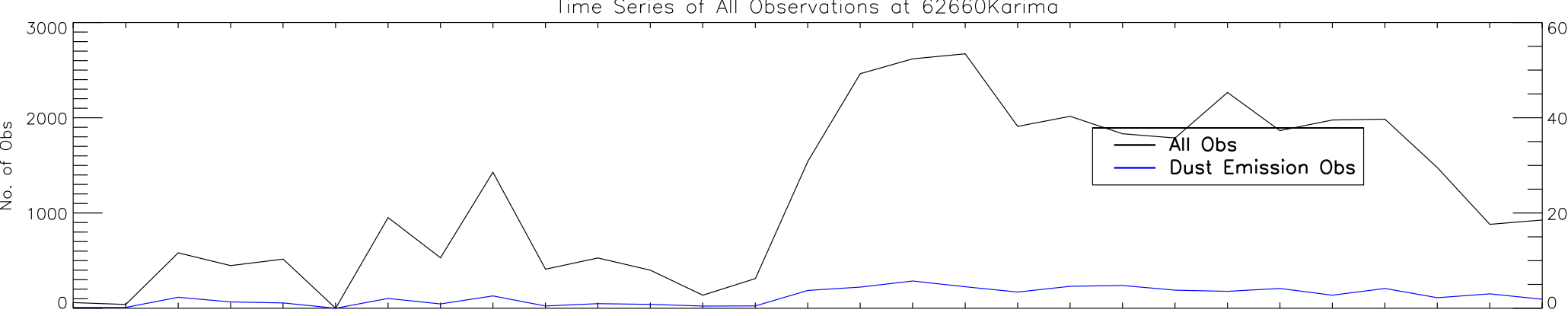

19841985198619871988198919901991199219931994199519961997199819992000200120022003200420052006200720082009201020112012

Seasonal Time Series of All Observations at 62660Karima

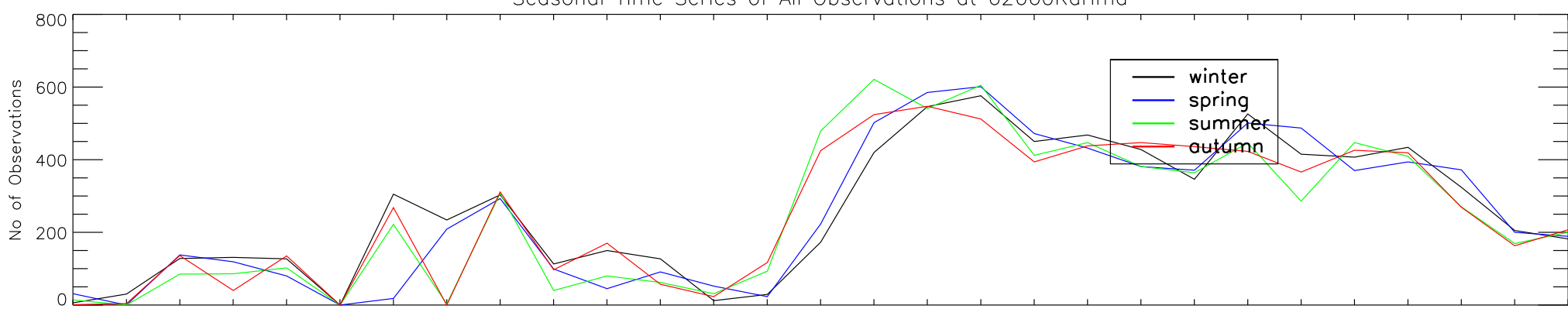

19841985198619871988198919901991199219931994199519961997199819992000200120022003200420052006200720082009201020112012

Seasonal Time Series of Dust Observations at 62660Karima

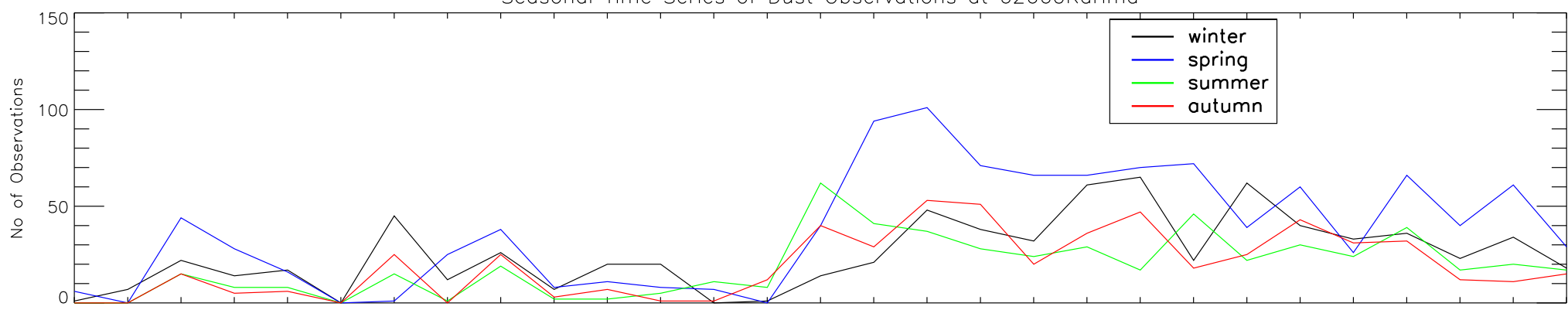

19841985198619871988198919901991199219931994199519961997199819992000200120022003200420052006200720082009201020112012 Time 
Time Series of All Observations at 62680Atbara

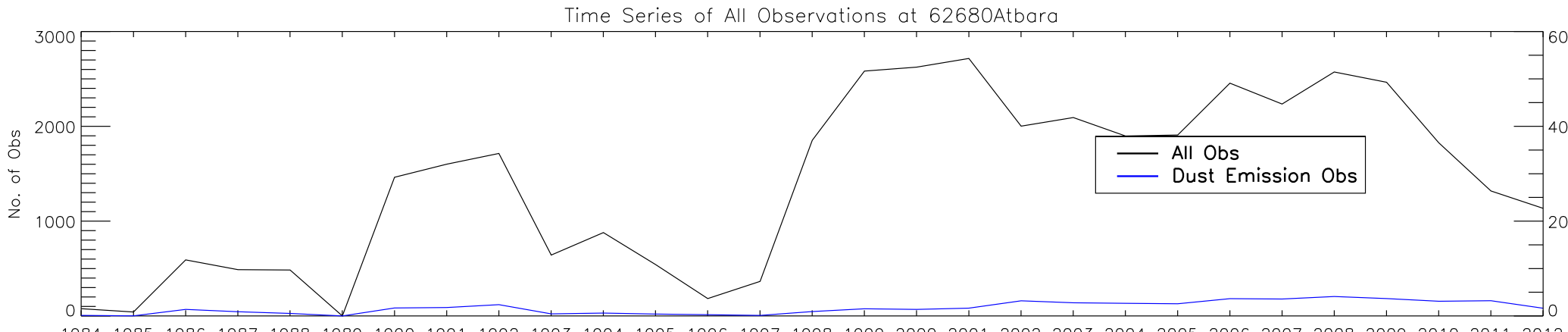

19841985198619871988198919901991199219931994199519961997199819992000200120022003200420052006200720082009201020112012 Time

Seasonal Time Series of All Observations at 62680Atbara

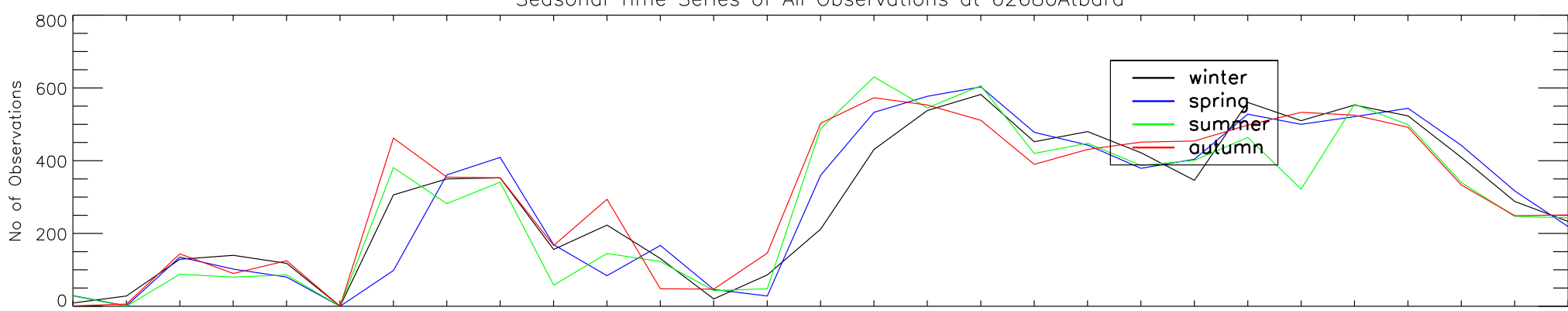

19841985198619871988198919901991199219931994199519961997199819992000200120022003200420052006200720082009201020112012 Time

Seasonal Time Series of Dust Observations at 62680Atbara

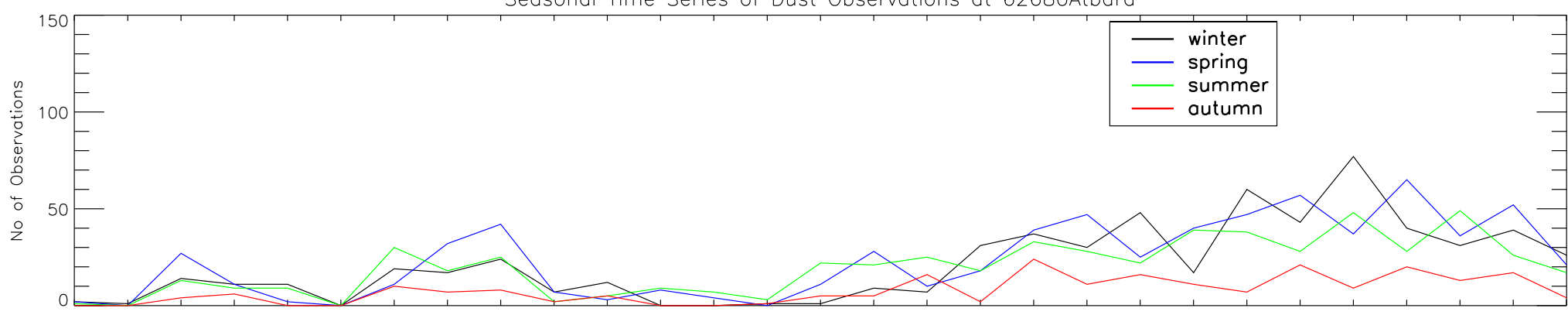

19841985198619871988198919901991199219931994199519961997199819992000200120022003200420052006200720082009201020112012 Time 
 \\ 3000}

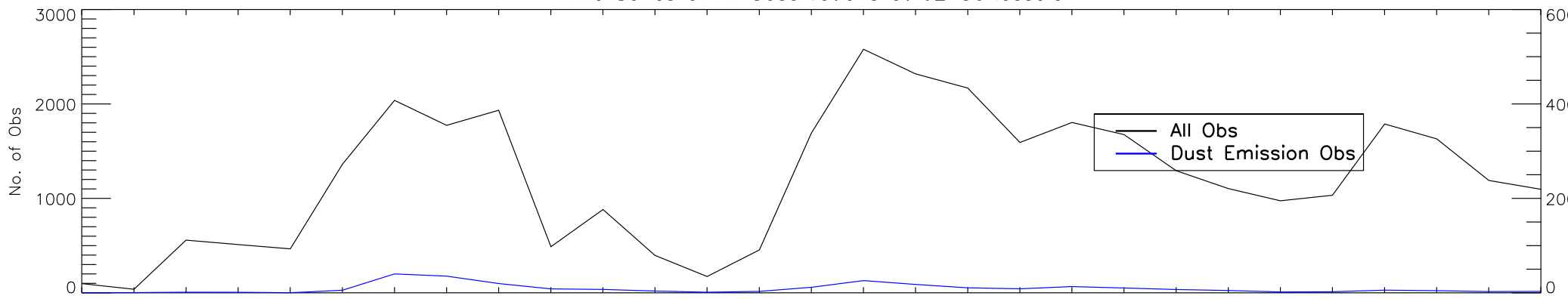

19841985198619871988198919901991199219931994199519961997199819992000200120022003200420052006200720082009201020112012 Time

Seasonal Time Series of All Observations at 62730Kassala

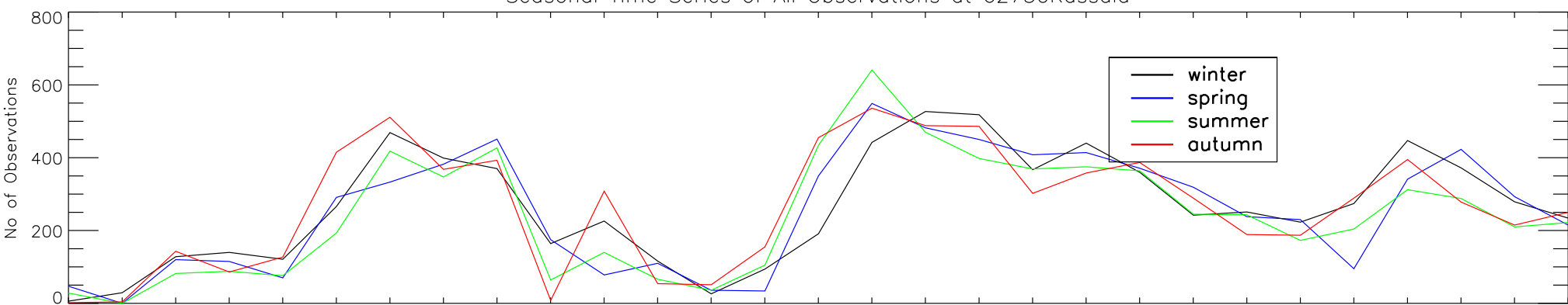

19841985198619871988198919901991199219931994199519961997199819992000200120022003200420052006200720082009201020112012

Seasonal Time Series of Dust Observations at 62730Kassala

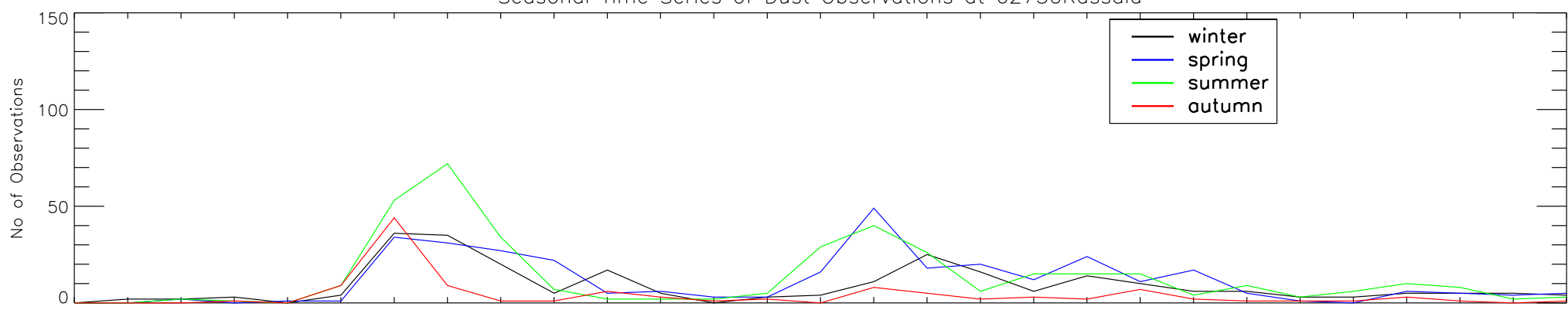

19841985198619871988198919901991199219931994199519961997199819992000200120022003200420052006200720082009201020112012 Time 


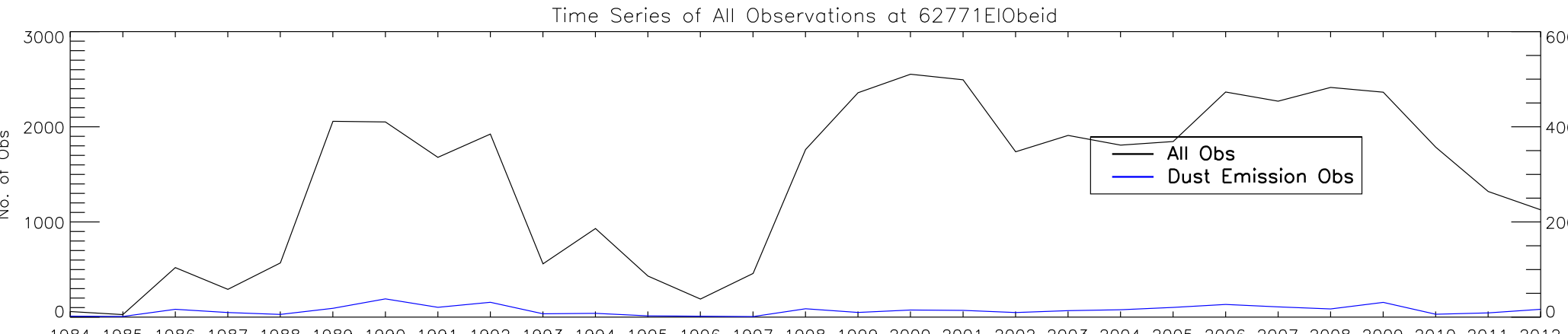

19841985198619871988198919901991199219931994199519961997199819992000200120022003200420052006200720082009201020112012 Time

Seasonal Time Series of All Observations at 62771ElObeid

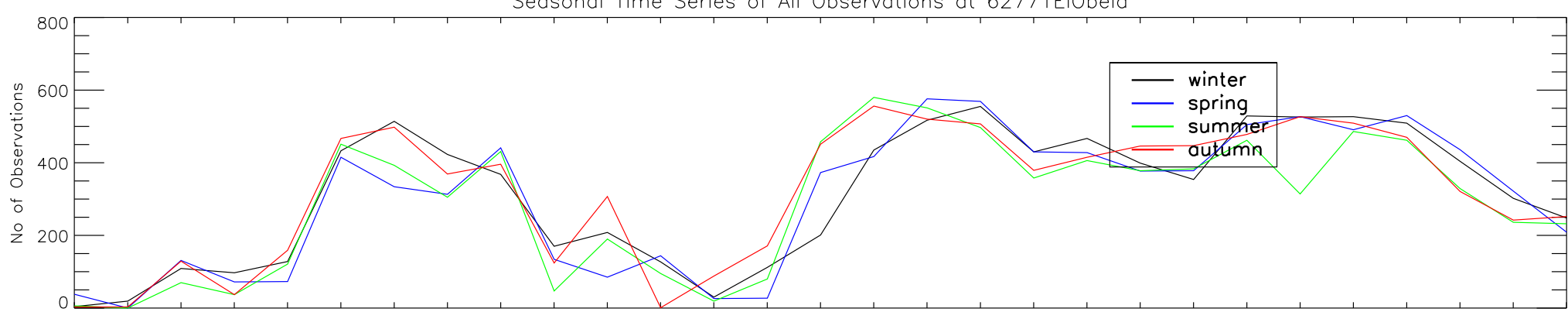

19841985198619871988198919901991199219931994199519961997199819992000200120022003200420052006200720082009201020112012

Seasonal Time Series of Dust Observations at 62771ElObeid

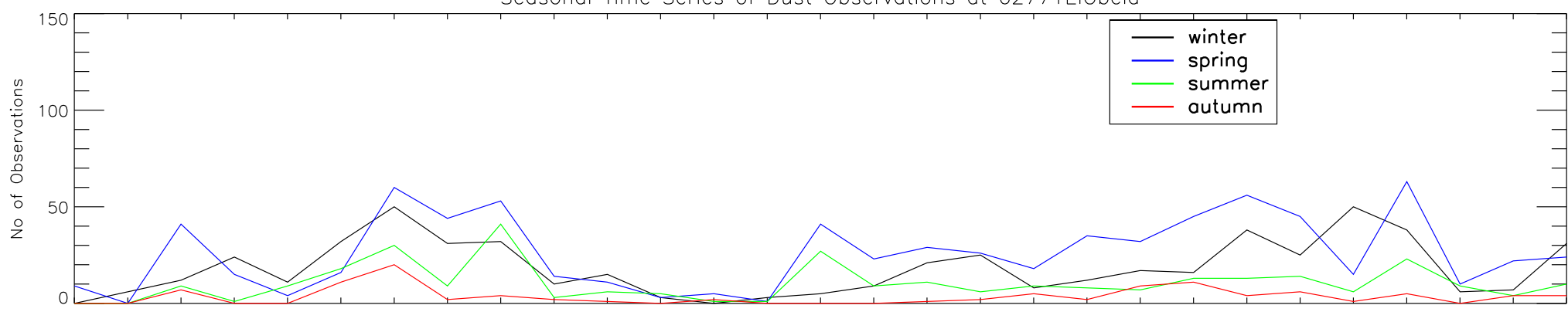

19841985198619871988198919901991199219931994199519961997199819992000200120022003200420052006200720082009201020112012 Time 
Time Series of All Observations at 62790Nyala

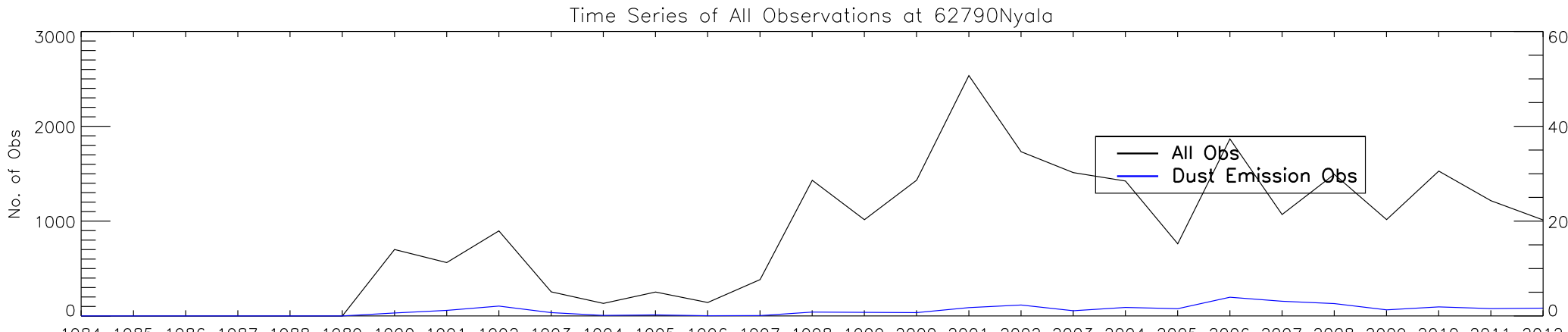

19841985198619871988198919901991199219931994199519961997199819992000200120022003200420052006200720082009201020112012 Time

Seasonal Time Series of All Observations at 62790Nyala

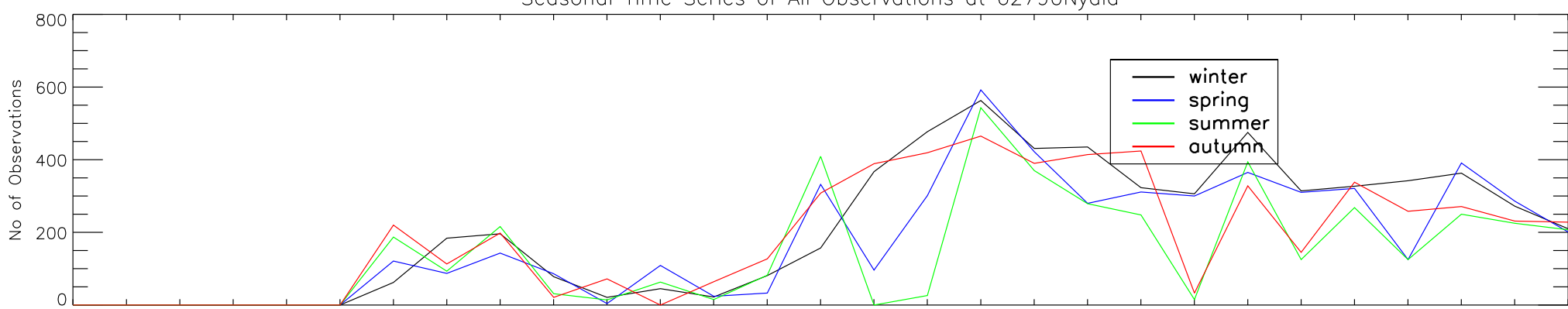

19841985198619871988198919901991199219931994199519961997199819992000200120022003200420052006200720082009201020112012

Seasonal Time Series of Dust Observations at 62790Nyala

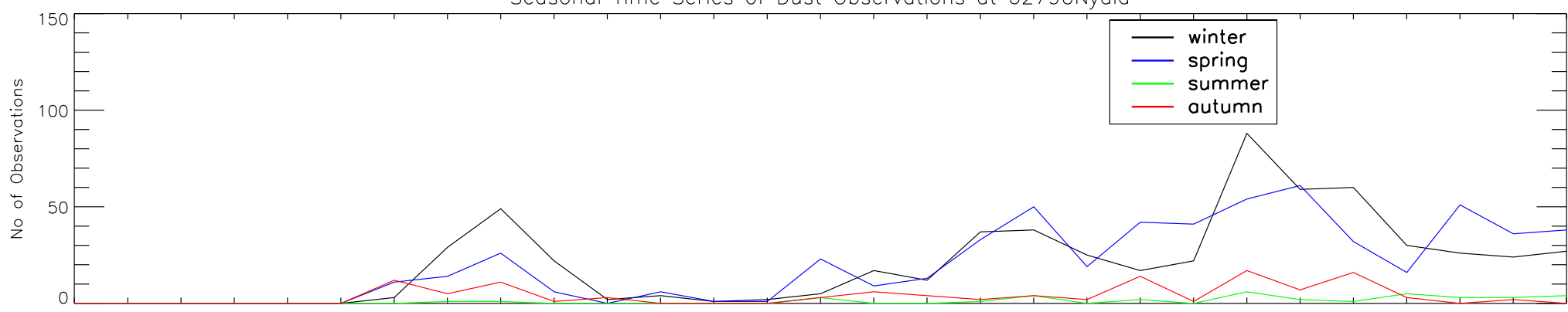

19841985198619871988198919901991199219931994199519961997199819992000200120022003200420052006200720082009201020112012 Time 
Time Series of All Observations at 64753Faya

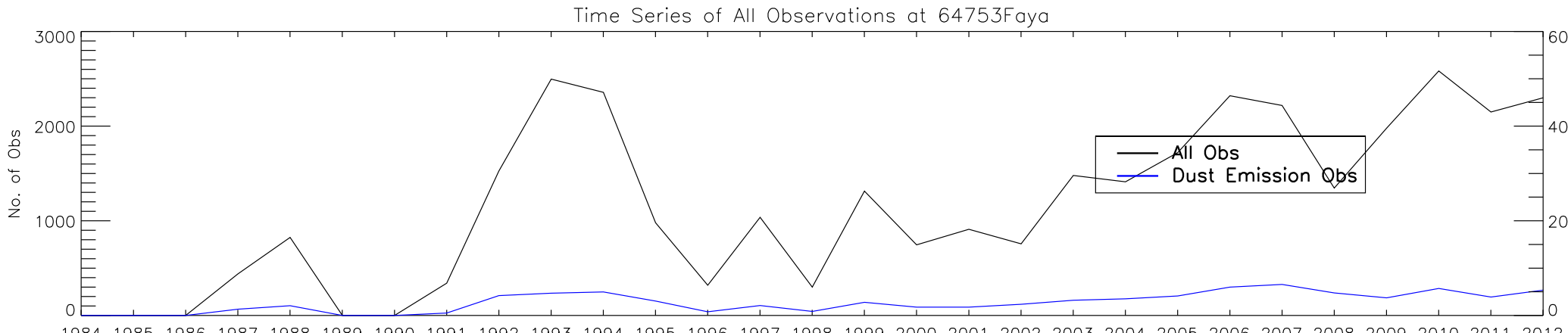

19841985198619871988198919901991199219931994199519961997199819992000200120022003200420052006200720082009201020112012 Time

Seasonal Time Series of All Observations at 64753Faya

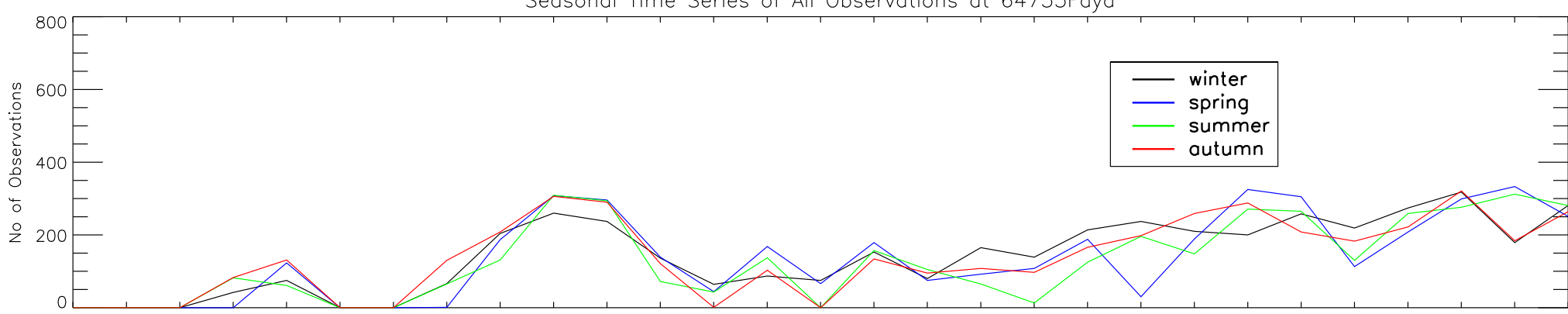

19841985198619871988198919901991199219931994199519961997199819992000200120022003200420052006200720082009201020112012

Seasonal Time Series of Dust Observations at 64753Faya

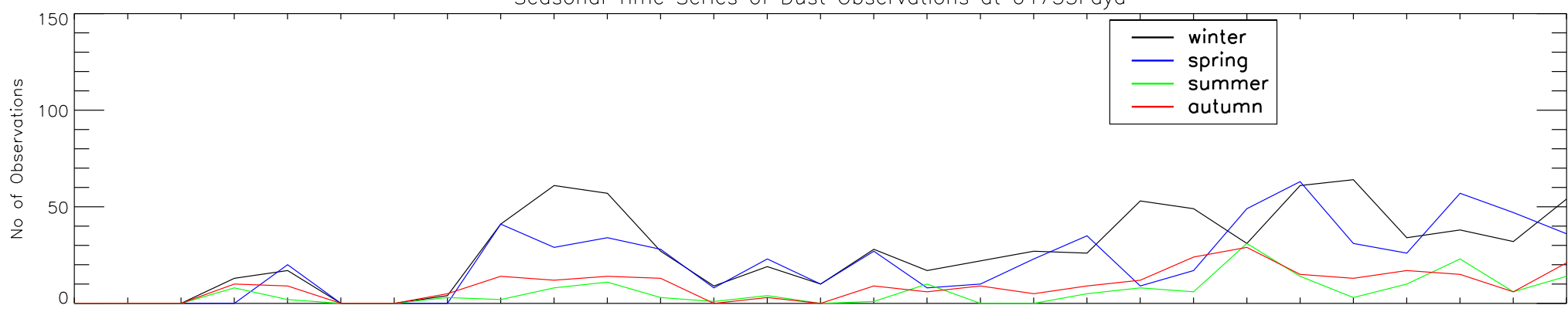

19841985198619871988198919901991199219931994199519961997199819992000200120022003200420052006200720082009201020112012 Time 
Figure S3. Annual time series of all observations (black line) and dust observations only (blue line; top plot). Seasonal time series of all observations (middle plot) where $\mathrm{DJF}=$ winter(black), MAM=spring(blue), JJA=summer(green), SON=(autumn). The bottom panel is the same as the middle, but for dust observations only. 

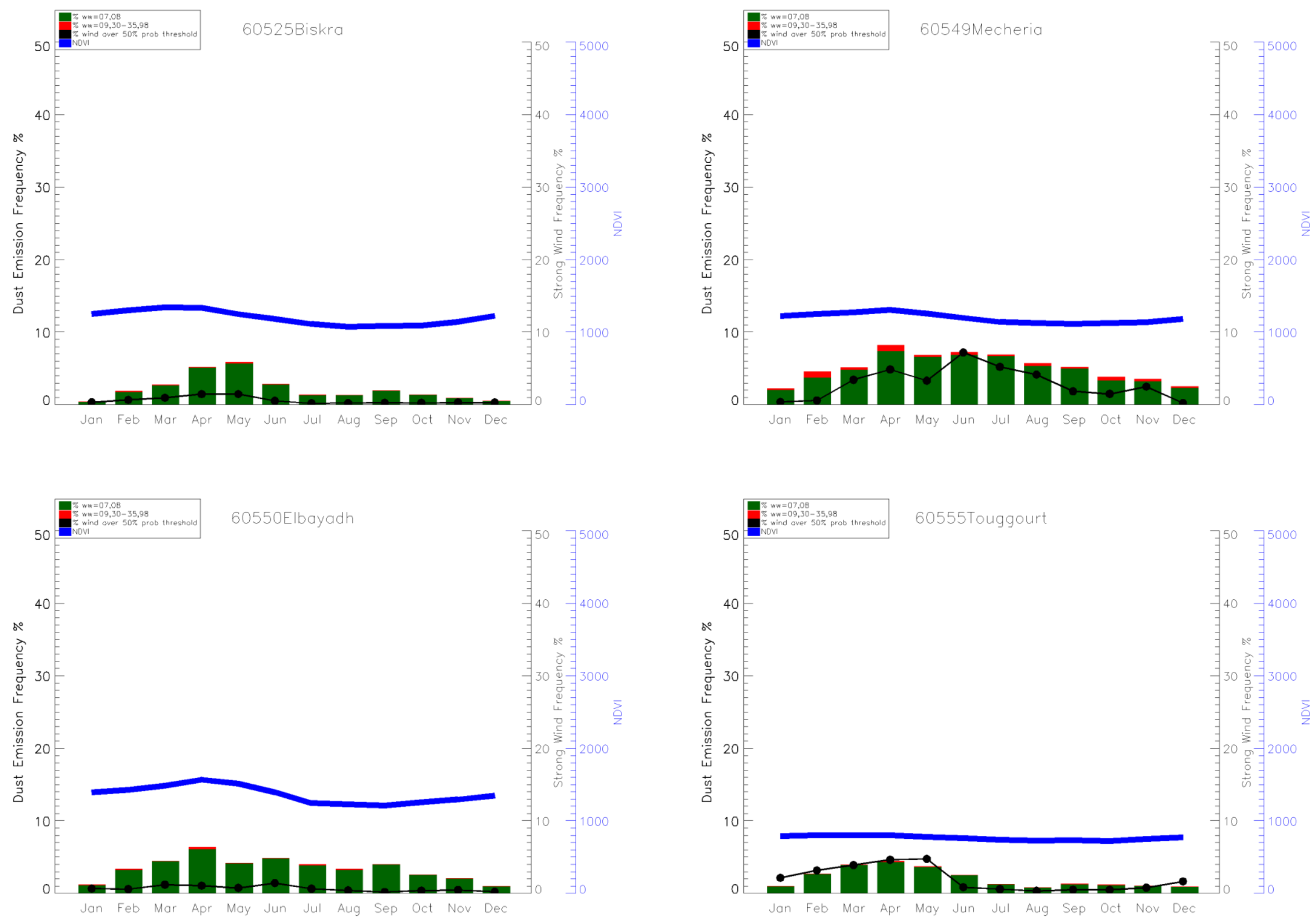

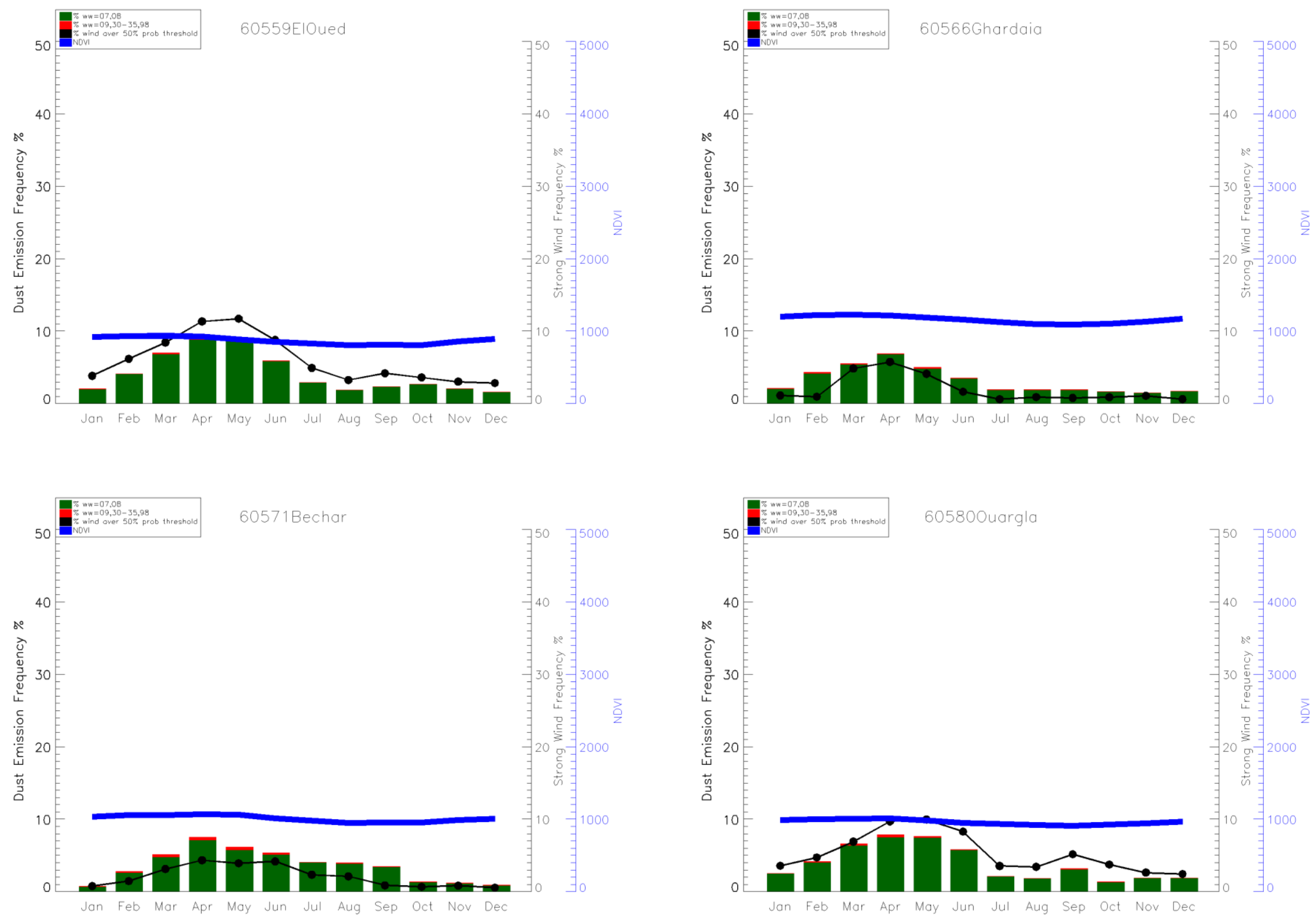

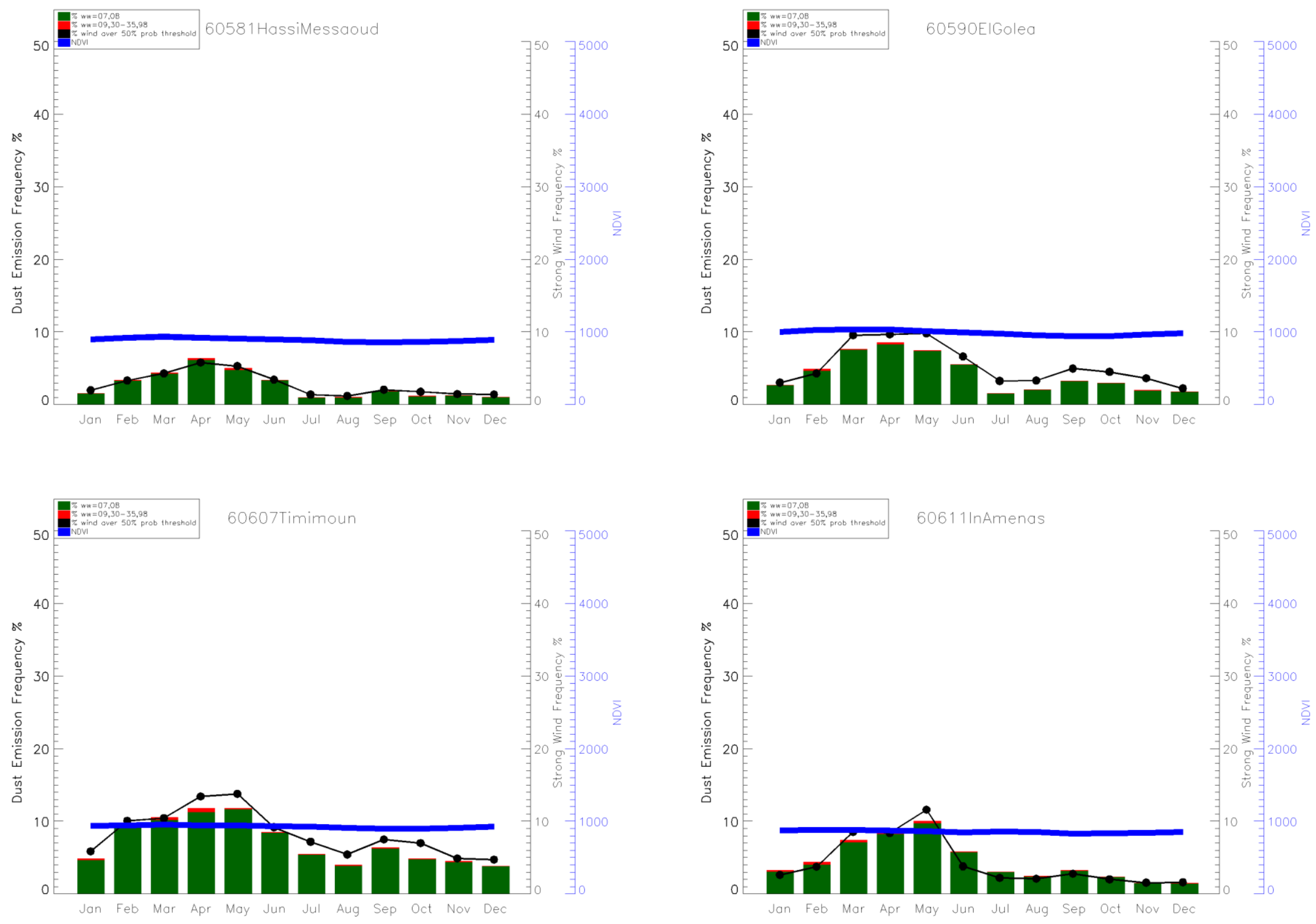

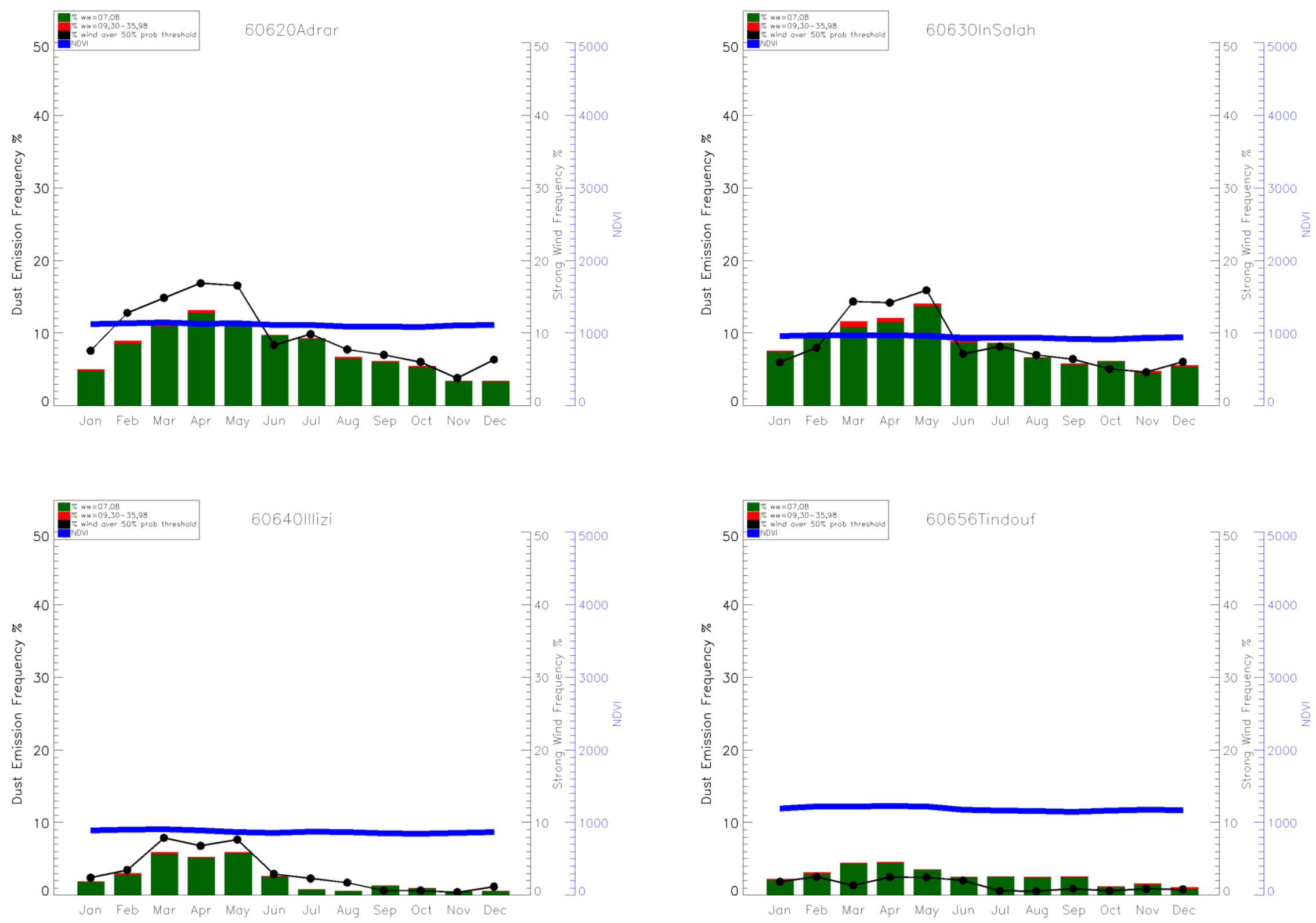

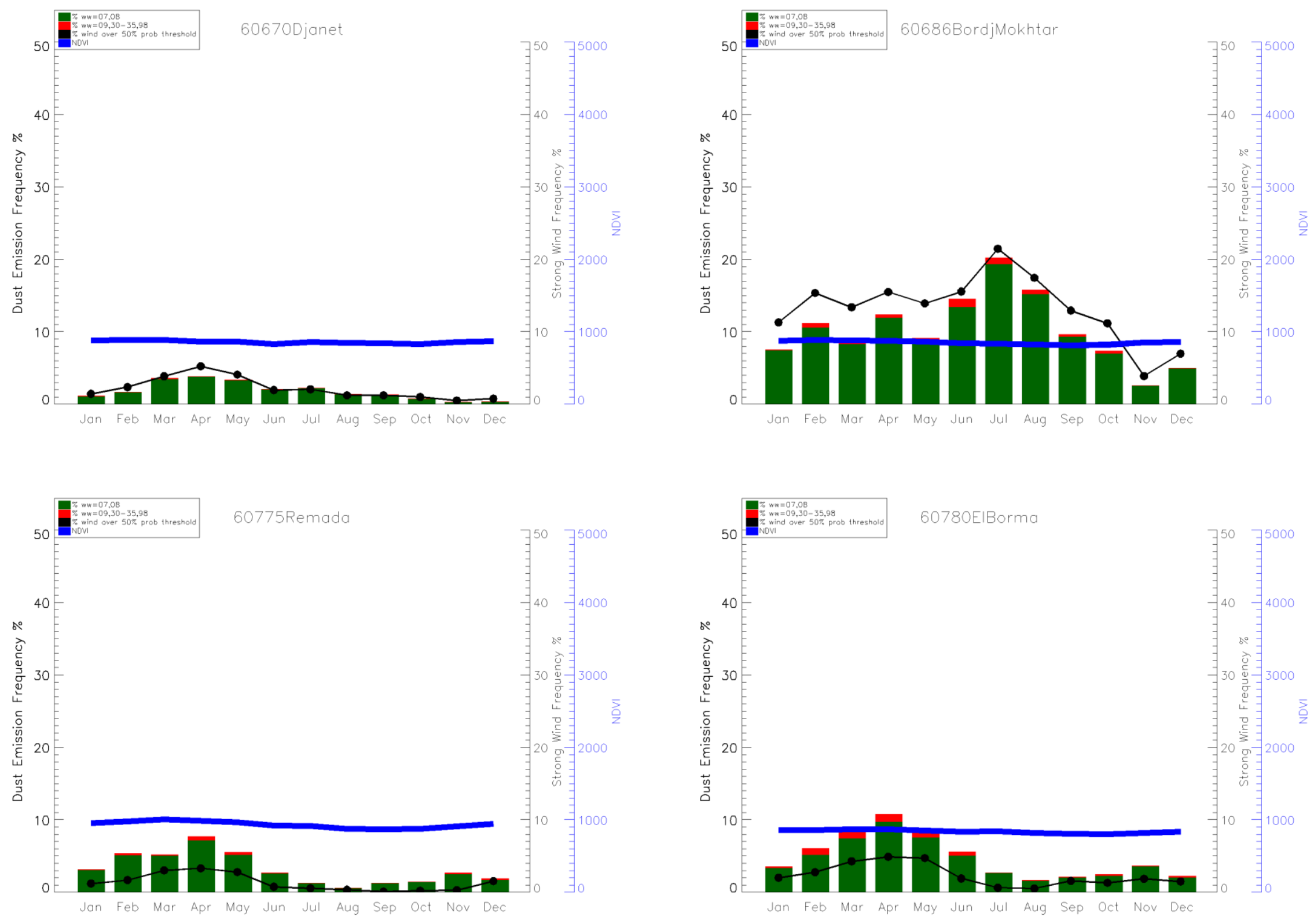

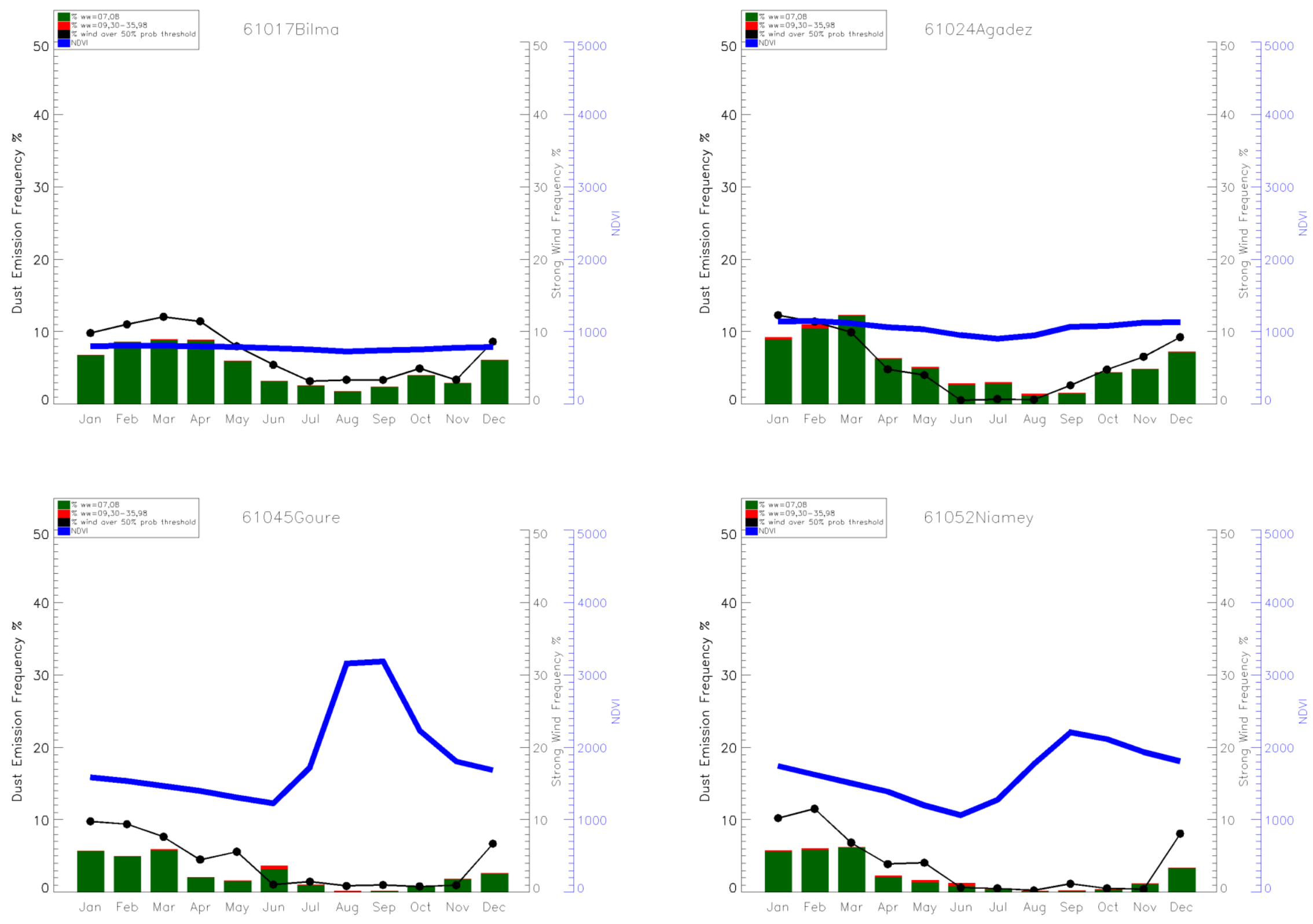

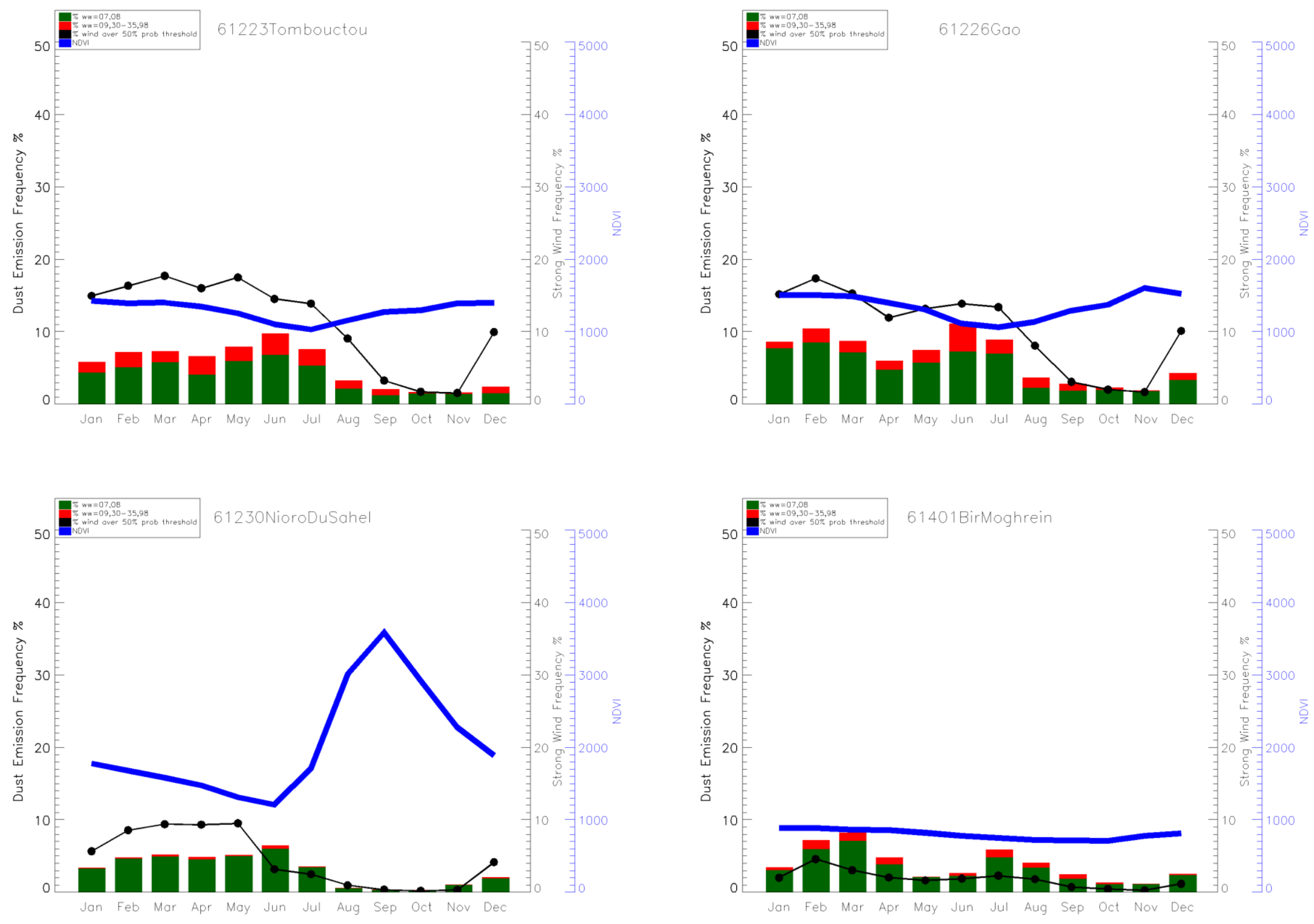

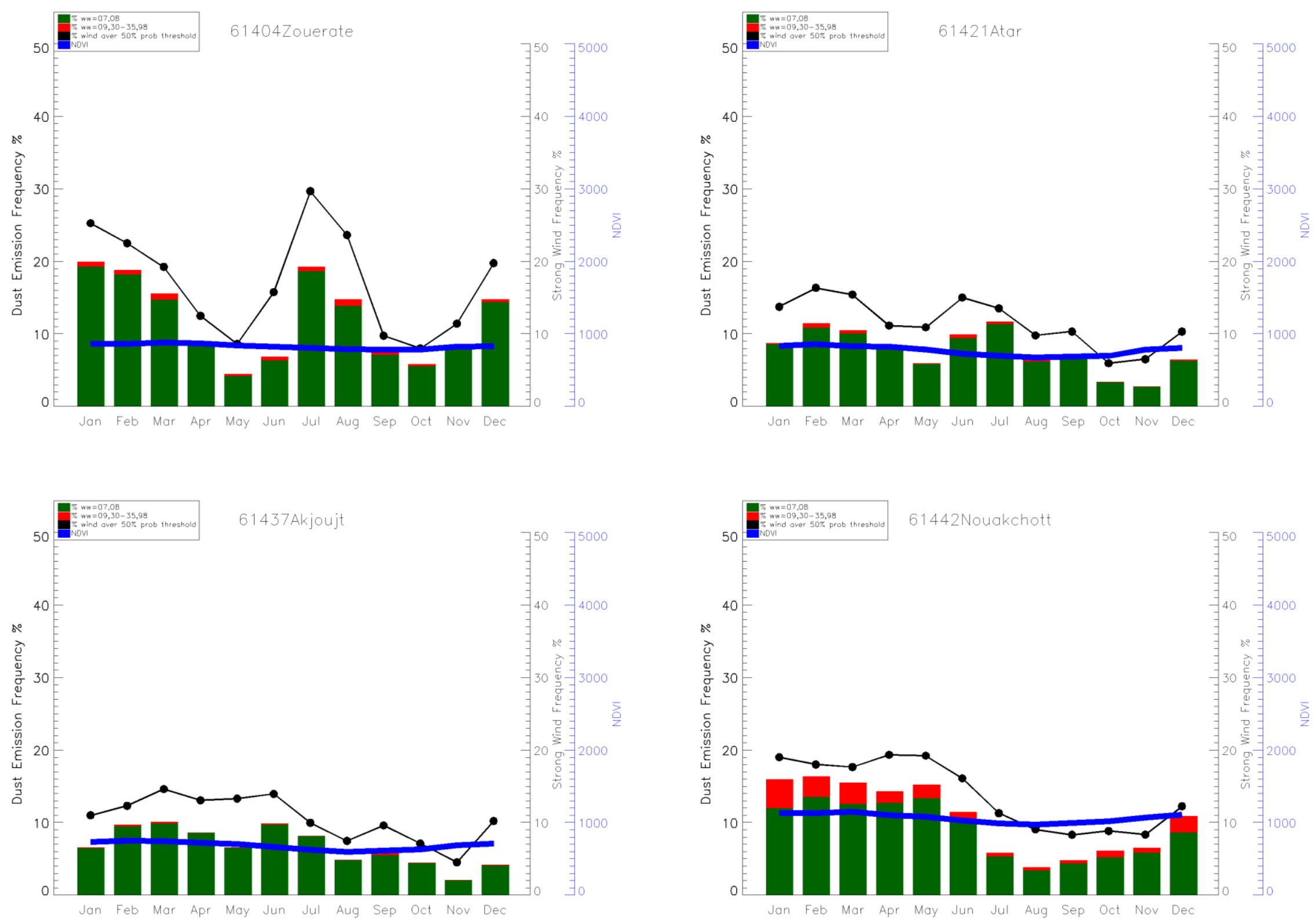

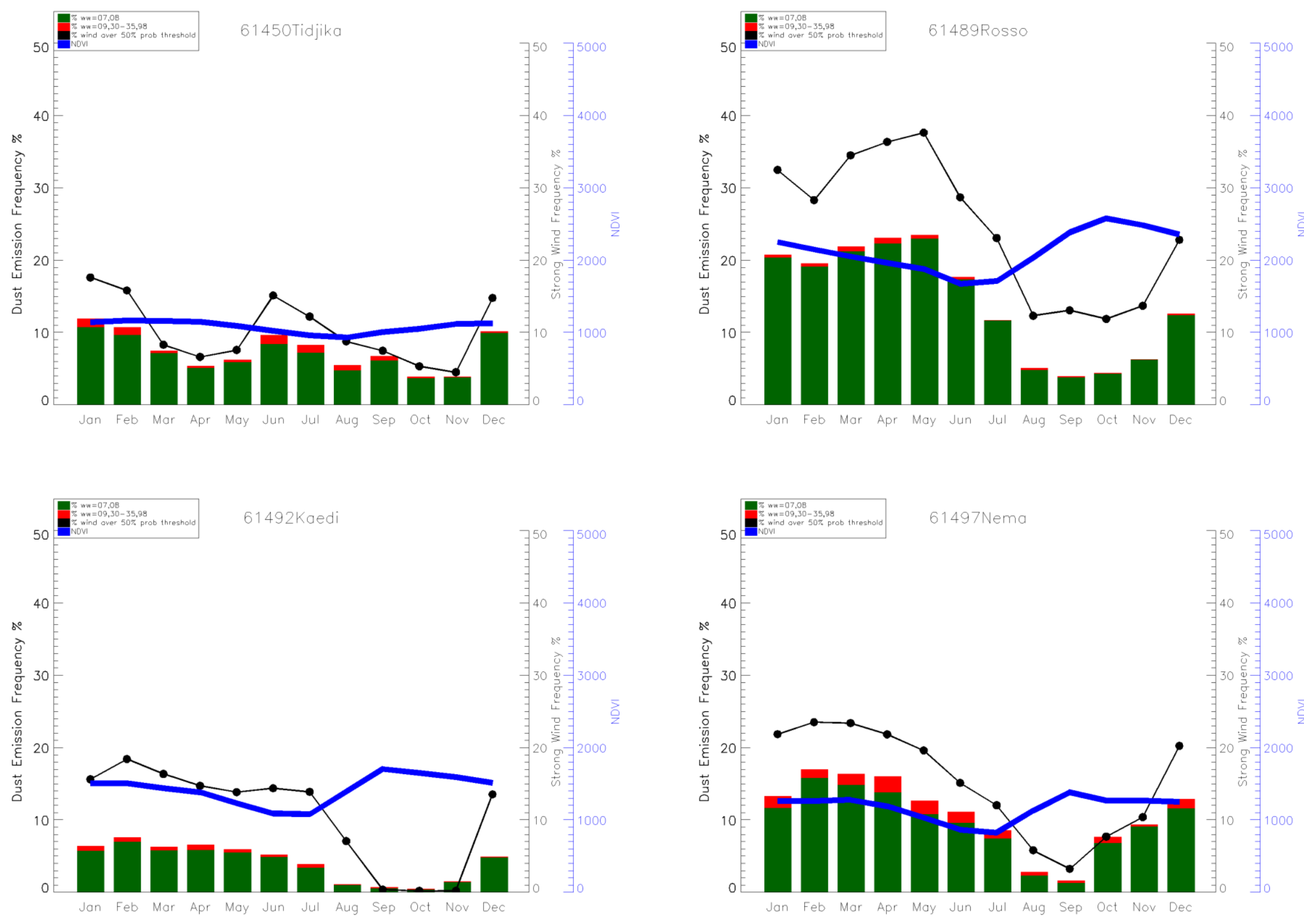

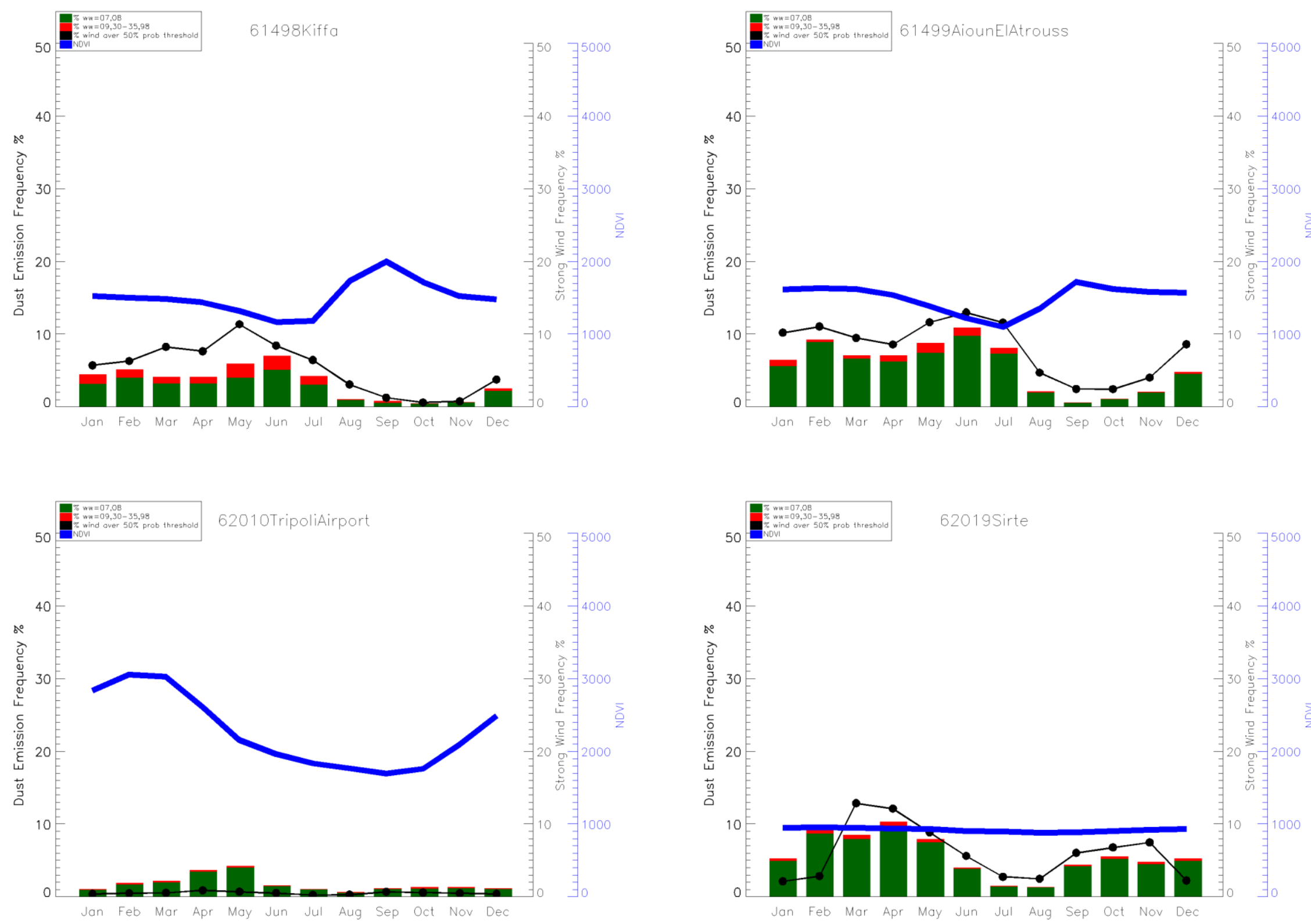

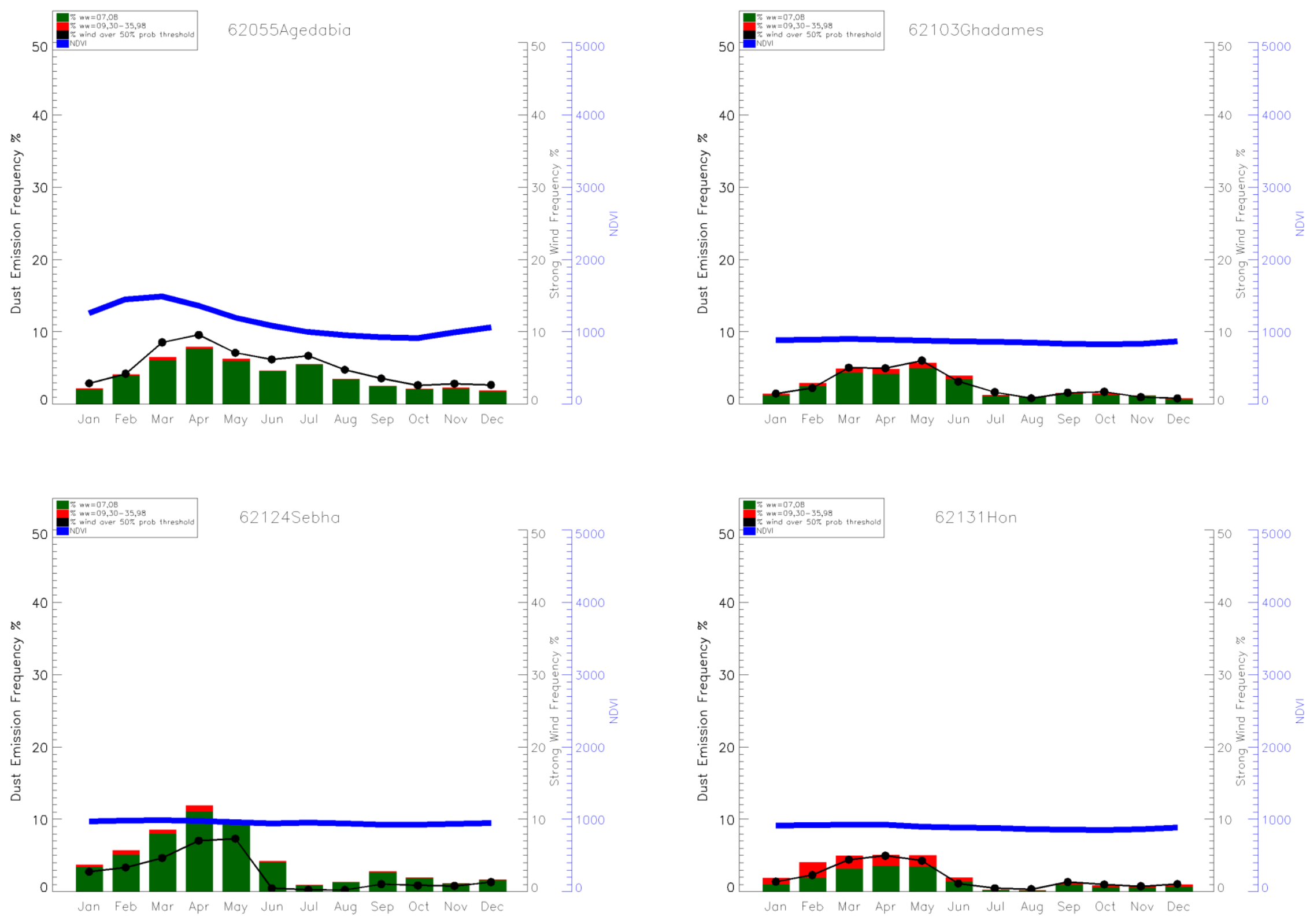

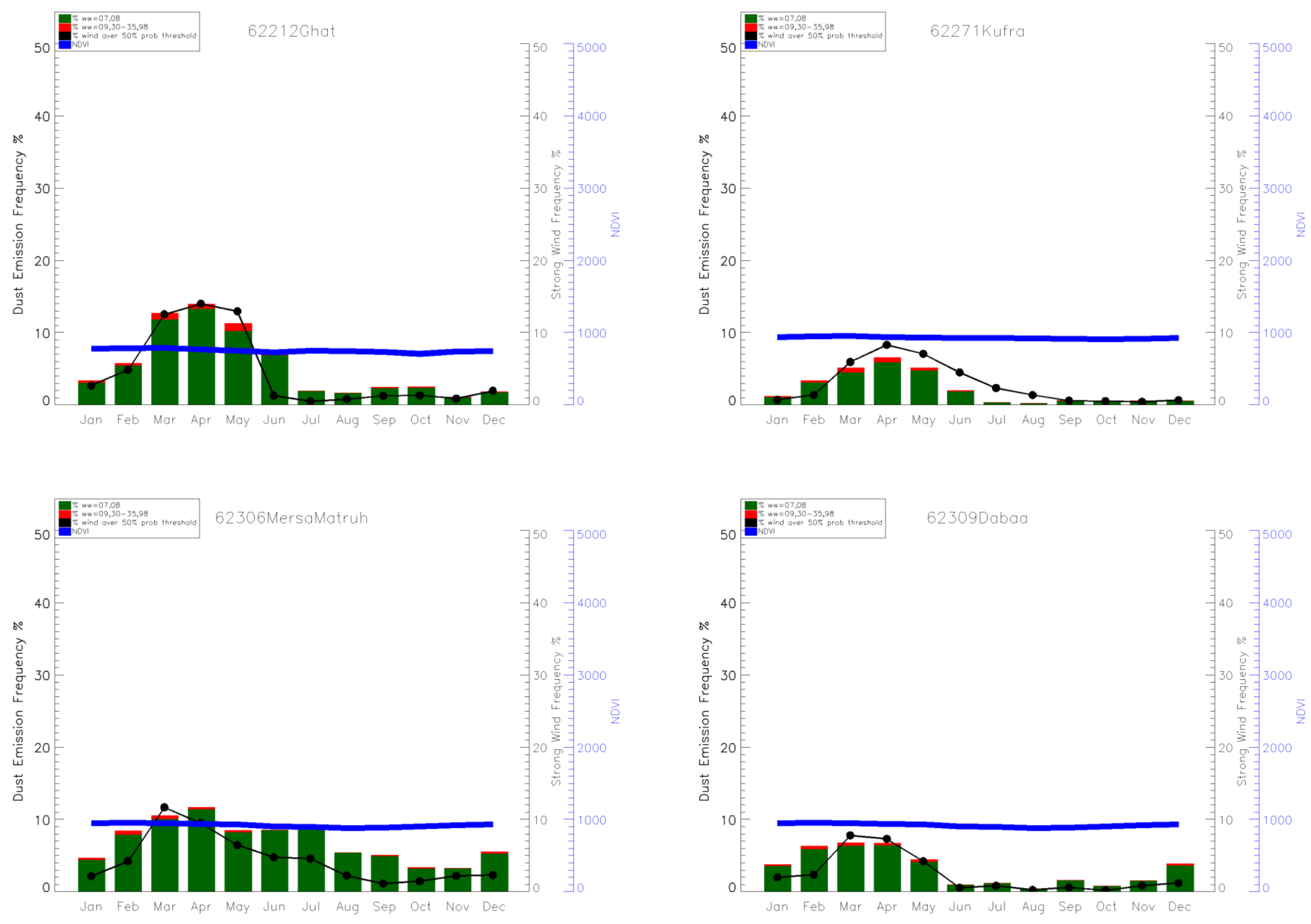

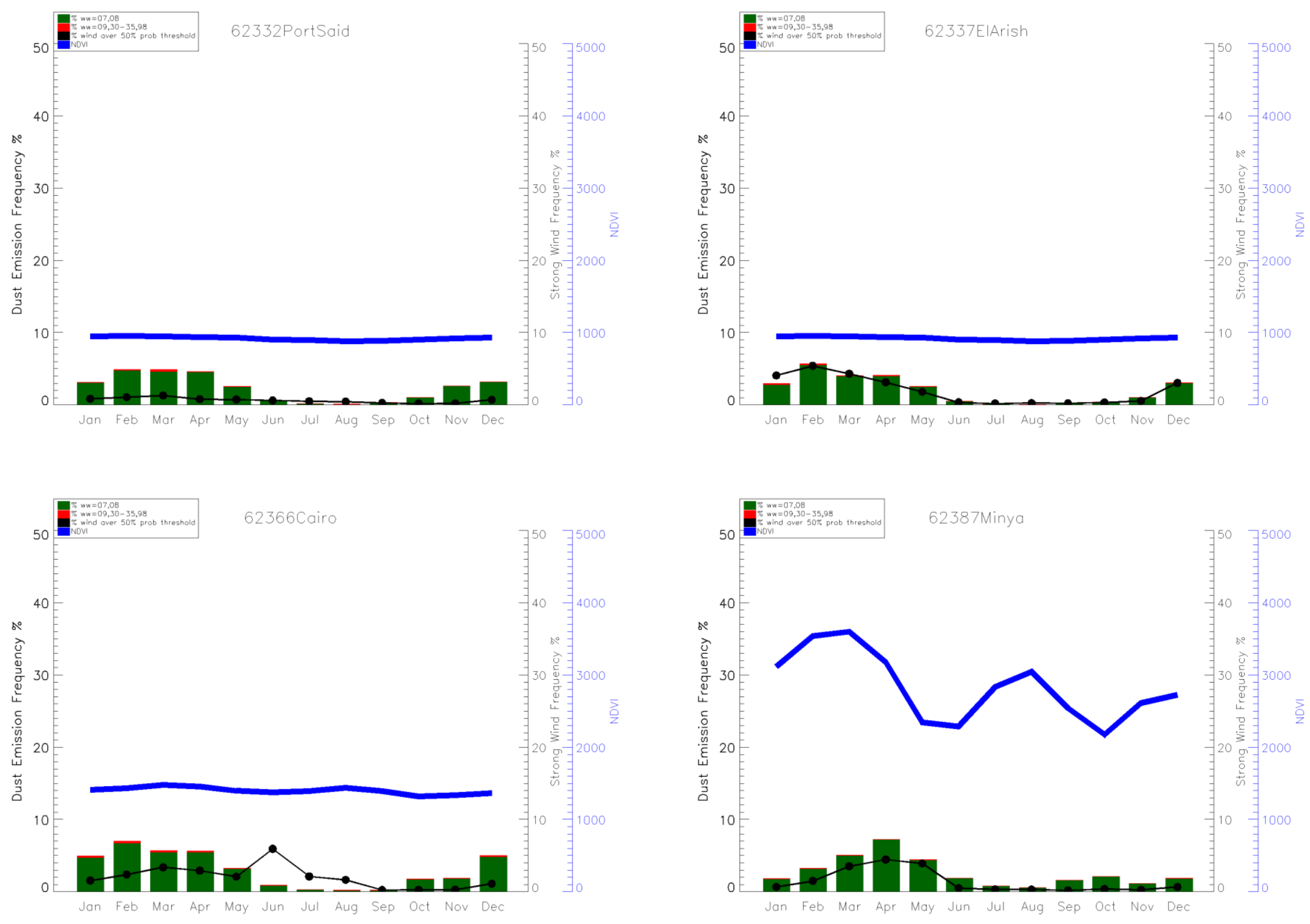

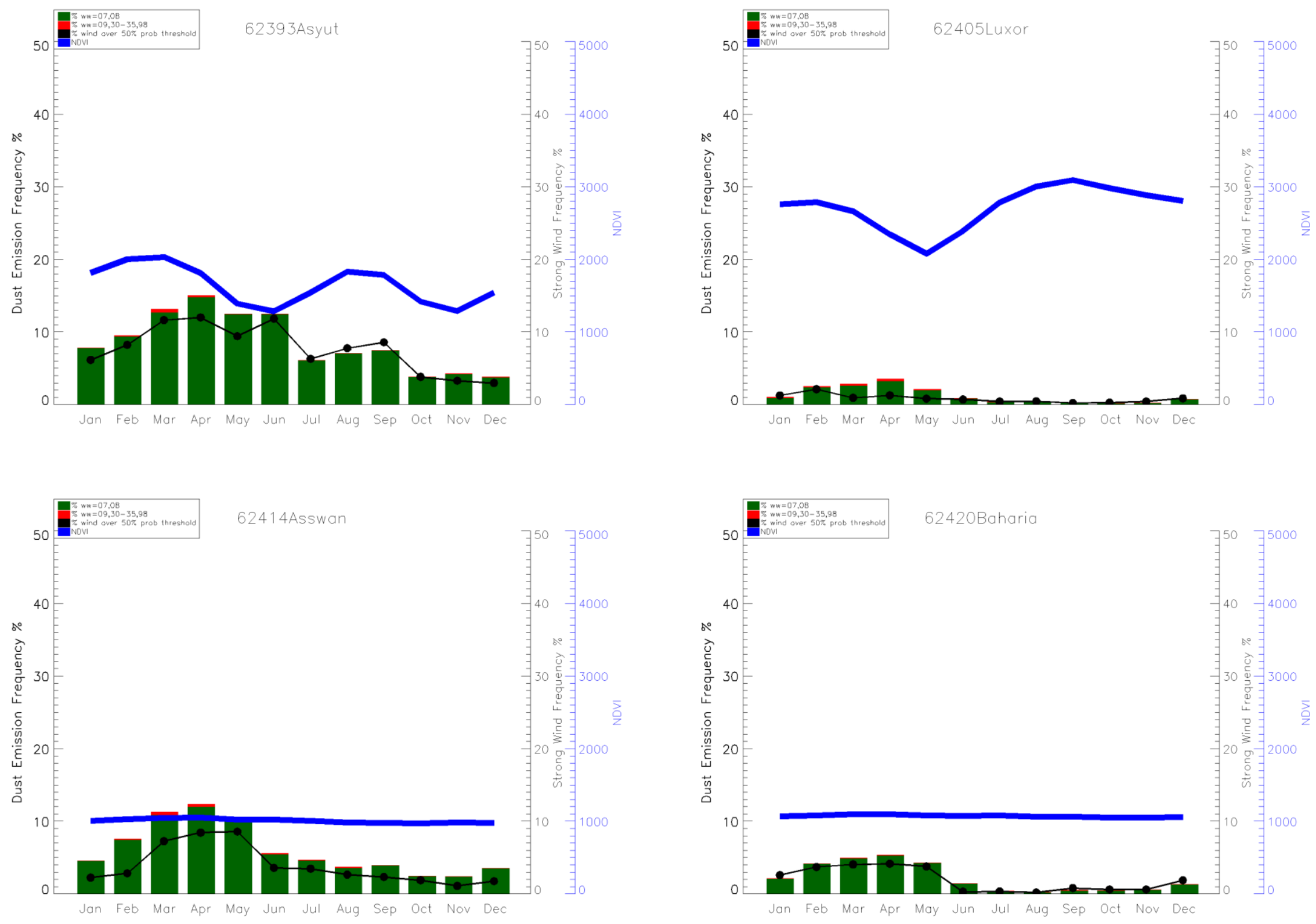

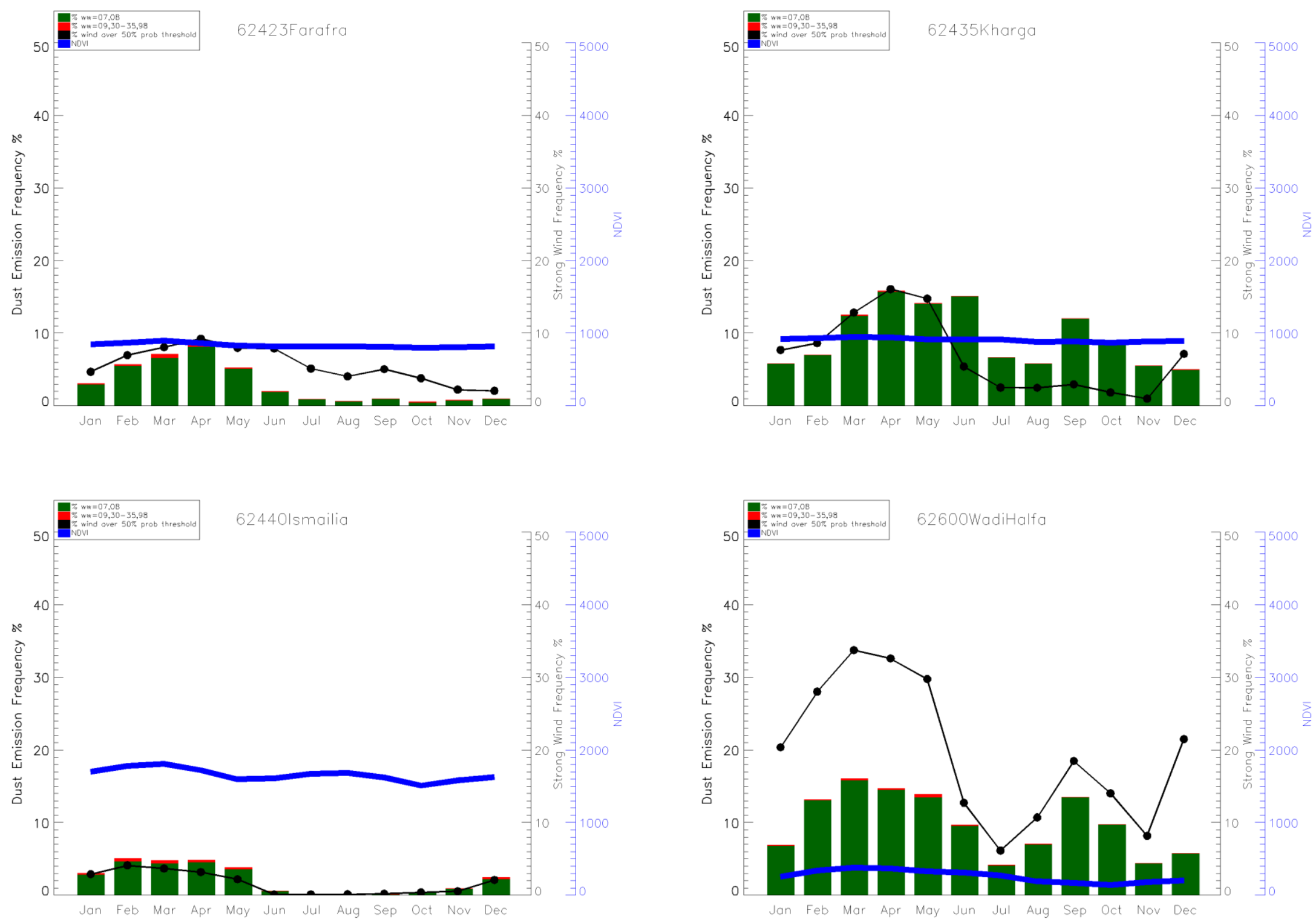

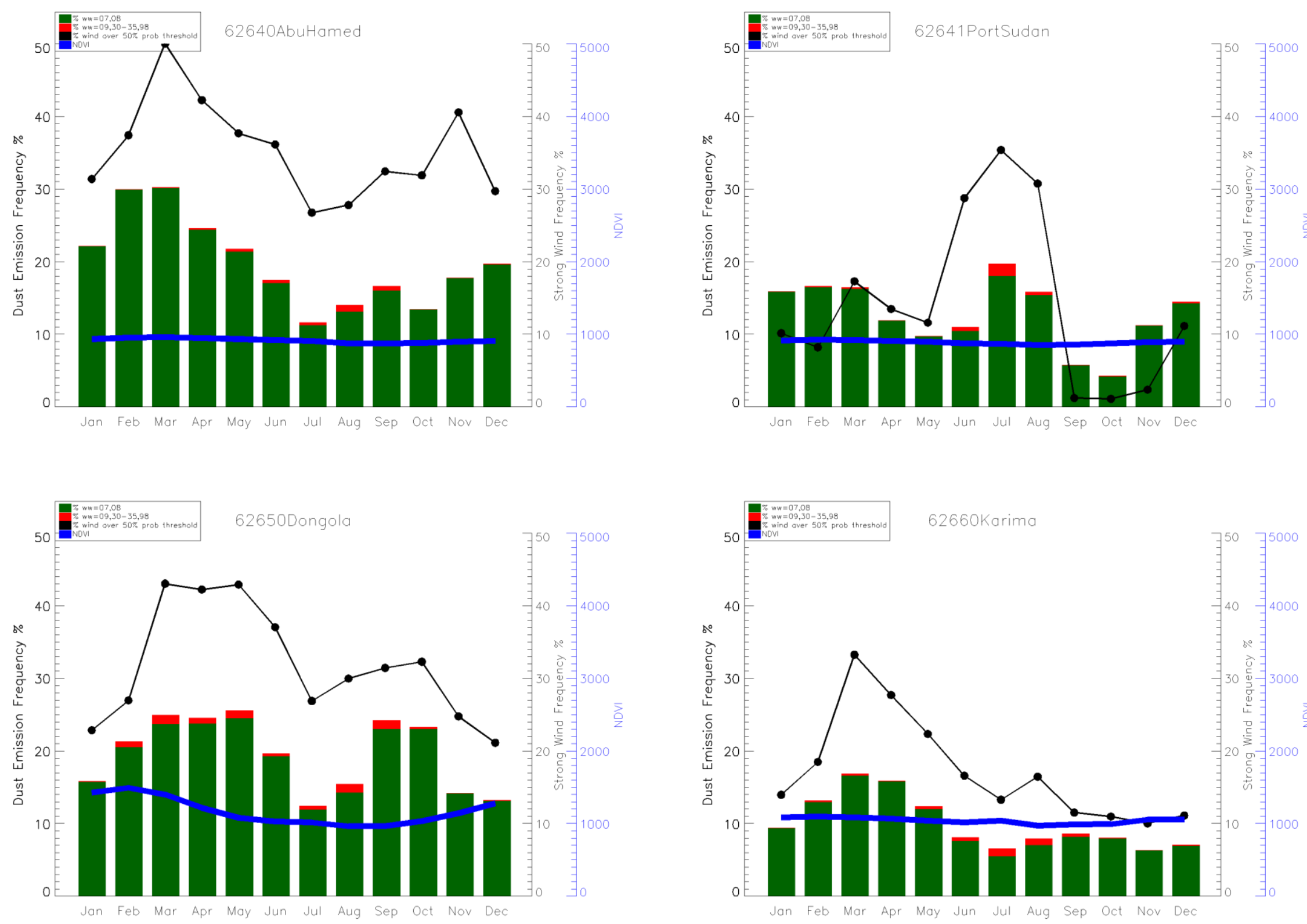

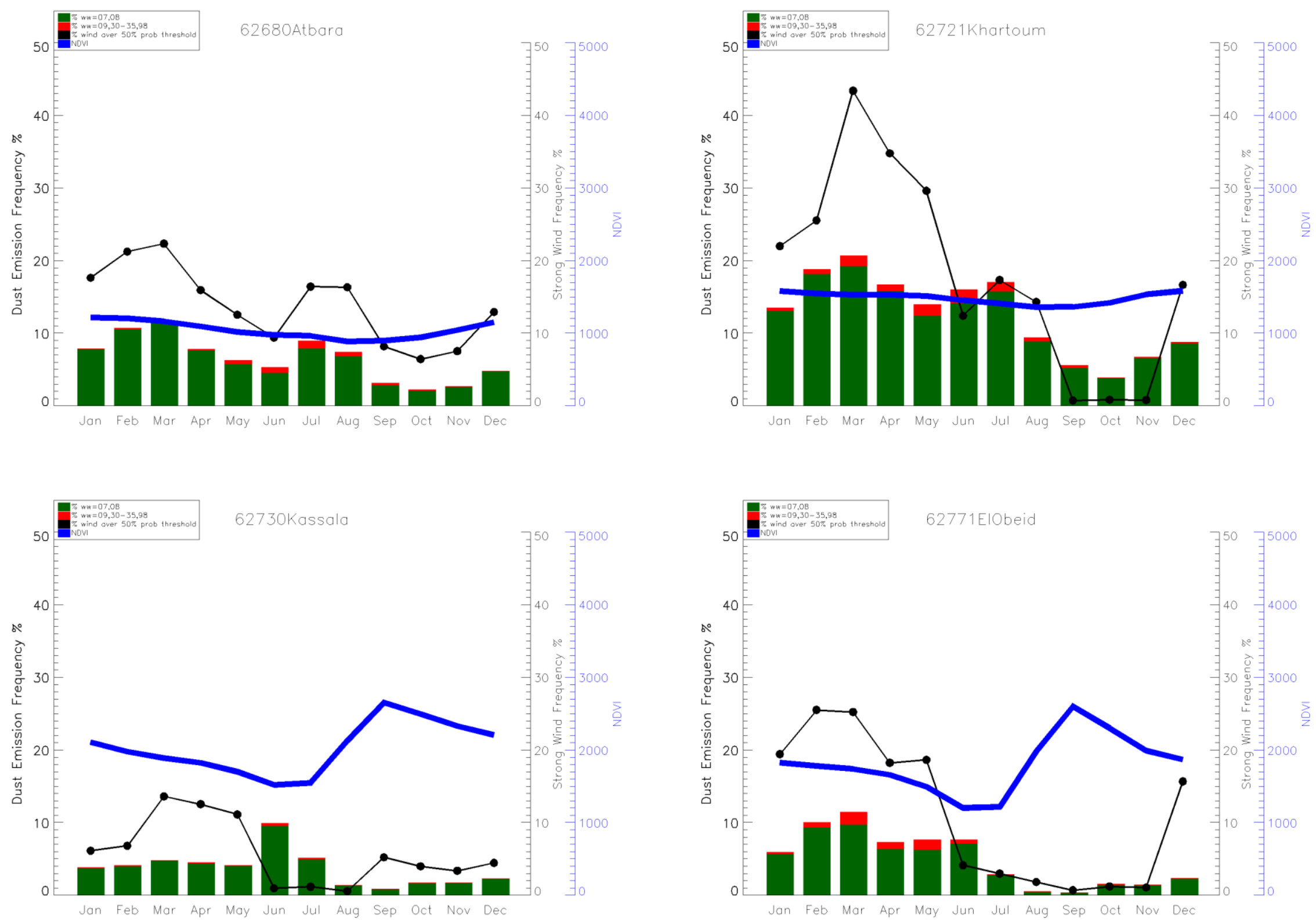

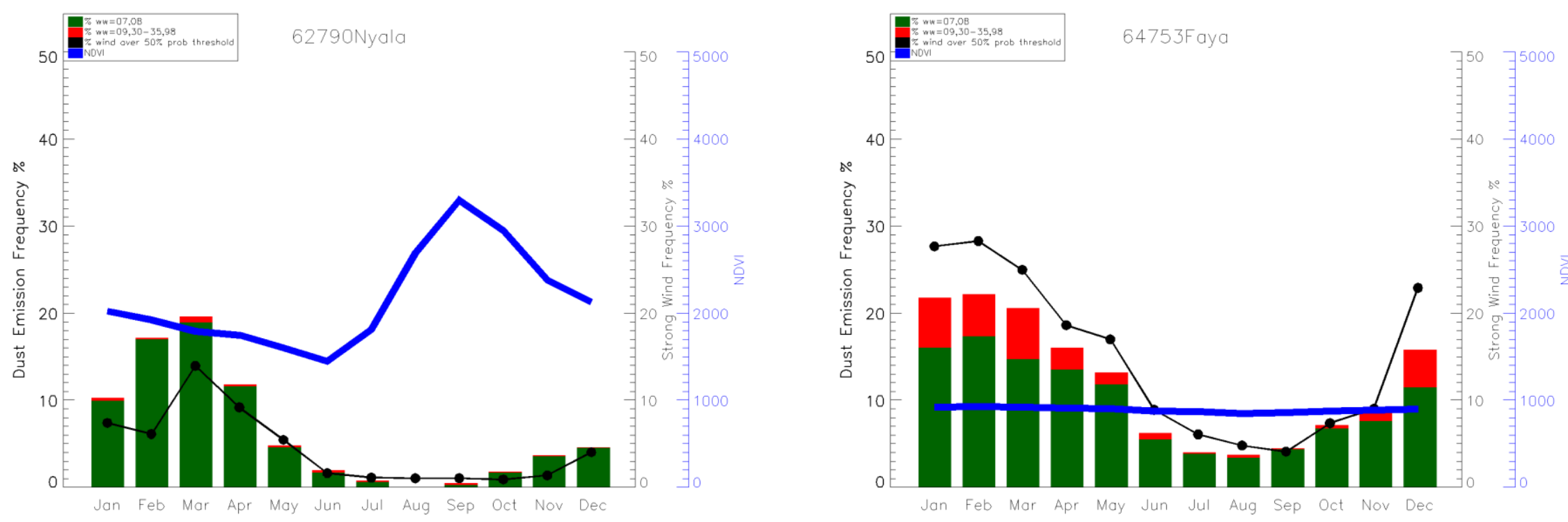

Figure S4. Mean seasonal cycle of frequency of dust emission (FDE) and strong wind frequency (SWF) for each of the 70 stations. Monthly mean FDE is split into BDF (green bars) and DSF (red bars) (for definitions, see main paper section 2.1.1), as well as monthly mean NDVI (blue lines) calculated for a $24 \mathrm{~km} \times 24 \mathrm{~km}$ box centred on each station. Strong wind frequency (SWF; black dotted line) is calculated for winds over T50 (see main paper, section 2.2.1) at each station. 